\title{
Sending a Bivariate Gaussian Source Over a Gaussian MAC with Unidirectional Conferencing Encoders
}

\author{
Shraga I. Bross and Yaron Laufer
}

\begin{abstract}
We consider the transmission of a memoryless bivariate Gaussian source over a two-user additive Gaussian multiple-access channel with unidirectional conferencing encoders. Here, prior to each transmission block, Encoder 1, which observes the first source component, is allowed to communicate with Encoder 2, which observes the second source component, via a unidirectional noise-free bit-pipe of given capacity. The main results of this work are sufficient conditions and a necessary condition for the achievability of a distortion pair expressed as a function of the channel SNR and of the source correlation. The main sufficient condition is obtained by an extension of the vector-quantizer scheme suggested by Lapidoth-Tinguely, for the case without conferencing, to the case with unidirectional conference. In the high-SNR regime, and when the capacity of the conference channel is unlimited, these necessary and sufficient conditions are shown to agree. We evaluate the precise high-SNR asymptotics for a subset of distortion pairs when the capacity of the conference channel is unlimited in which case we show that a separation based scheme attains these optimal distortion pairs. However, with symmetric average-power constraints and fixed conferencing capacity, at high-SNR the latter separation based scheme is shown to be suboptimal.
\end{abstract}

Keywords - Joint source-channel coding, Gaussian multiple-access channel, unidirectional conferencing encoders.

\section{InTROdUCtion AND PROBlem STATEMENT}

We consider a communication scenario where two encoders transmit a memoryless bivariate Gaussian source to a single receiver over a two-user additive white Gaussian multiple-access channel (MAC). The source is observed separately by the two encoders; Encoder 1 observes the first source component and Encoder 2 observes the second source component. The encoders are allowed to partially cooperate in the sense that prior to each transmission block, Encoder 1 is allowed to communicate with Encoder 2 via a unidirectional noise-free bit-pipe of given capacity, as shown in Fig. 1 Both encoders then cooperate in describing the source components to a common receiver, via an average-power constrained Gaussian MAC. From the output of the multiple-access channel, the receiver wishes to reconstruct each source component with the least possible expected squared-error distortion. Our interest is in characterizing the distortion pairs that are simultaneously achievable on the two source components. Special cases are the classical MAC considered by Lapidoth-Tinguely in [1], where the encoders are ignorant of each others inputs (the bit-pipe is of strictly zero capacity -i.e. no connection at all) and the asymmetric setting, where Encoder 2 is fully cognizant of the source input at Encoder 1 (the bit-pipe is of infinite capacity).

S. Bross is with the Engineering Department, Bar-Ilan University, Ramat Gan 52900, Israel. Email:brosss@ biu.ac.il. The work of S. Bross was supported by the Israel Science Foundation under Grant 497/09 and Grant 455/14.

Y. Laufer is with the Engineering Department, Bar-Ilan University, Ramat Gan 52900, Israel. Email:yaron_laufer@walla.com. 
Henceforth, we adopt the following notation conventions. Random variables will be denoted by capital letters, while their realizations will be denoted by the respective lower case letters. Whenever the dimension of a random vector is clear from the context the random vector will be denoted by a bold face letter, that is, $\mathbf{X}$ denotes the random vector $\left(X_{1}, X_{2}, \ldots, X_{n}\right)$, and $\mathbf{x}=\left(x_{1}, x_{2}, \ldots, x_{n}\right)$ will designate a specific sample value of $\mathbf{X}$. The alphabet of a scalar random variable $X$ will be designated by a calligraphic letter $\mathcal{X}$. The $n$-fold Cartesian power of a generic alphabet $\mathcal{V}$, that is, the set of all $n$-vectors over $\mathcal{V}$, will be denoted $\mathcal{V}^{n}$. An estimator of a random variable $X$ is denoted by $\hat{X}$. For a real-valued parameter $0 \leq \beta \leq 1$ we define $\bar{\beta} \triangleq 1-\beta$, and for a nonnegative distortion constraint $D$ the corresponding normalized distortion is defined by $d \triangleq D / \sigma^{2}$ where $\sigma^{2}$ is the source variance.

Formally, the time- $k$ output of the Gaussian MAC is given by

$$
Y_{k}=x_{1, k}+x_{2, k}+Z_{k},
$$

where $\left(x_{1, k}, x_{2, k}\right) \in \mathbb{R}^{2}$ are the symbols sent by the transmitters, and $Z_{k}$ is the time- $k$ additive noise term. The sequence $\left\{Z_{k}\right\}$ consists of independent identically distributed (IID) zero-mean variance $N$ Gaussian random variables that are independent of the source sequence.

The input source sequence $\left\{\left(S_{1, k}, S_{2, k}\right)\right\}$ consists of zero-mean Gaussians of covariance

$$
\mathrm{K}_{S S}=\left(\begin{array}{cc}
\sigma^{2} & \rho \sigma^{2} \\
\rho \sigma^{2} & \sigma^{2}
\end{array}\right)
$$

with $\rho \in[0,1]$, and $0<\sigma^{2}<\infty$ (for a justification for the restriction to $\rho \in[0,1]$ and $\sigma_{1}^{2}=\sigma_{2}^{2}=\sigma^{2}$ see [1, Section II.C]).

Note: There are just two exceptions to the notation conventions defined above. Throughout this work we define several scalings of the source correlation coefficient. Specifically, we define $\tilde{\rho}$ and $\bar{\rho}$ as per (19) (in which case $\bar{\rho} \neq 1-\rho$ ), and similarly $\hat{\rho}$ as per (21) (in which case $\hat{\rho}$ does not refer to an estimator of $\rho$ ).

The sequence $\left\{S_{1, k}\right\}$ is observed by Encoder 1 and the sequence $\left\{S_{2, k}\right\}$ is observed by Encoder 2. Prior to each block of $n$ channel uses, the encoders may exchange information via the use of the unidirectional bit-pipe which is assumed to be:

- perfect in the sense that any input symbol is available immediately and error-free at the output of the pipe; and

- of limited capacity $C_{12}$, in the sense that when the input to the pipe from Encoder 1 to Encoder 2 takes values in the set $\mathcal{W}$, such that $W=f^{(n)}\left(\mathbf{S}_{1}\right)$ for some encoding function $f^{(n)}: \mathbb{R}^{n} \mapsto \mathcal{W}$, then

$$
\log |\mathcal{W}| \leq n C_{12} .
$$

We define an $\left(n, C_{12}\right)$-conference to be a collection of an input alphabet $\mathcal{W}$, and an encoding function $f^{(n)}(\cdot)$ as above, where $n, C_{12}$ and the alphabet set satisfy (3).

After the conference, Encoder 2 is cognizant of the random variable $W$ so the channel inputs $\mathbf{X}_{1}=\left(X_{1,1}, \ldots, X_{1, n}\right)$ and $\mathbf{X}_{2}=\left(X_{2,1}, \ldots, X_{2, n}\right)$ can be described via encoding functions $\varphi_{1}^{(n)}$ and $\varphi_{2}^{(n)}$ as

$$
\begin{aligned}
& \mathbf{X}_{1}=\varphi_{1}^{(n)}\left(\mathbf{S}_{1}\right), \\
& \mathbf{X}_{2}=\varphi_{2}^{(n)}\left(\mathbf{S}_{2}, W\right)=\varphi_{2}^{(n)}\left(\mathbf{S}_{2}, f^{(n)}\left(\mathbf{S}_{1}\right)\right),
\end{aligned}
$$

where

$$
\begin{aligned}
& \varphi_{1}^{(n)}: \mathbb{R}^{n} \mapsto \mathbb{R}^{n}, \\
& \varphi_{2}^{(n)}: \mathbb{R}^{n} \times \mathcal{W} \mapsto \mathbb{R}^{n} .
\end{aligned}
$$




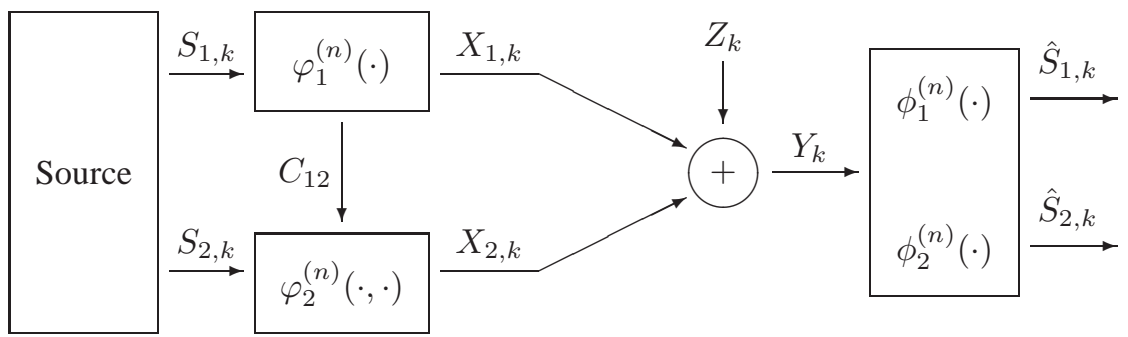

Fig. 1. Transmission of bivariate Gaussian source over a Gaussian multiple-access channel with unidirectional conferencing encoders.

The channel input sequences are average-power limited to $P_{1}$ and $P_{2}$ respectively, i.e.

$$
\frac{1}{n} \mathrm{E}\left[\sum_{k=1}^{n}\left(X_{\nu, k}\right)^{2}\right] \leq P_{\nu}, \quad \nu=1,2
$$

where $\mathrm{E}$ denotes the expectation operator. Based on the channel output $\mathbf{Y}=\left(Y_{1}, \ldots, Y_{n}\right)$ the receiver forms its estimates $\hat{\mathbf{S}}_{1}=\phi_{1}^{(n)}(\mathbf{Y})$ and $\hat{\mathbf{S}}_{2}=\phi_{2}^{(n)}(\mathbf{Y})$ for the source sequences respectively, where

$$
\phi_{\nu}^{(n)}: \mathbb{R}^{n} \mapsto \mathbb{R}^{n}, \quad \nu=1,2 .
$$

We are interested in the minimal expected squared-error distortions at which the receiver can reconstruct each of the source sequences.

Definition 1: Given $\sigma^{2}>0, \rho \in[0,1], P_{1}, P_{2}, N>0$ and $C_{12}>0$ we say that the distortion pair $\left(D_{1}, D_{2}\right)$ is achievable if there exists a sequence of block-lengths $n$, encoding functions $f^{(n)}$ that belong to an $\left(n, C_{12}\right)$-conference, encoders $\left(\varphi_{1}^{(n)}, \varphi_{2}^{(n)}\right)$ as in (5) satisfying the averagepower constraints (6), and reconstruction functions $\left(\phi_{1}^{(n)}, \phi_{2}^{(n)}\right)$ as in (7) resulting in average distortions that fulfill

$$
\varlimsup_{n \rightarrow \infty} \frac{1}{n} \sum_{k=1}^{n} \mathrm{E}\left[\left(S_{\nu, k}-\hat{S}_{\nu, k}\right)^{2}\right] \leq D_{\nu}, \quad \nu=1,2,
$$

whenever $Y_{k}=\varphi_{1, k}^{(n)}\left(\mathbf{S}_{1}\right)+\varphi_{2, k}^{(n)}\left(\mathbf{S}_{2}, f^{(n)}\left(\mathbf{S}_{1}\right)\right)+Z_{k}, k=1, \ldots, n$, and $\left\{\left(S_{1, k}, S_{2, k}\right)\right\}$ are IID zero-mean bivariate Gaussian vectors with covariance matrix $\mathrm{K}_{S S}$ as in (2) and $\left\{Z_{k}\right\}$ are IID zero-mean variance- $N$ Gaussian random variables that are independent of $\left\{\left(S_{1, k}, S_{2, k}\right)\right\}$.

In [3] the authors provided sufficient conditions for reliable transmission of correlated sources over a regular MAC and demonstrated that, in general, the separation approach is not optimal. For the regular MAC, the separation approach is known to be optimal when the channel is lossless (Slepian-Wolf source coding theorem [4]), or when the sources are independent. In the special case of transmitting correlated sources losslessly over an asymmetric MAC it is shown in [5] that necessary and sufficient conditions for reliable transmission do exist and, moreover, these conditions can be established by applying the separation approach. In [6] the authors consider the model [5] with a single distortion constraint namely, when $D_{1}=0$ (i.e. $\mathbf{S}_{1}$ is recovered losslessly at the receiver), and show that source-channel separation is optimal.

A lossy Gaussian version of the problem addressed by Cover-El Gamal-Salehi [3] has been considered in [1], wherein the power-versus-distortion tradeoff for the distributed transmission of a memoryless bivariate Gaussian source over a two-to-one average-power limited Gaussian MAC is considered. Necessary and sufficient conditions for the achievability of a distortion pair are presented and it is shown that if the channel signal-to-noise ratio (SNR) is below a 
certain threshold uncoded transmission is optimal. Furthermore, the authors derive the high-SNR asymptotics for a subset of distortion pairs and show that the source-channel vector-quantizer, by means of which they derive their sufficient condition, is optimal at high-SNR. In the symmetric case of equal average-power constraints and equal distortions this vector-quantizer outperforms source-channel separation at all SNR's.

Our problem is also related to the correlated sources with partially separated encoders sourcecoding problem [9], and to the Gaussian MAC with conferencing encoders channel-coding problem [10] (see also [12]). However, the above two problems are source/channel coding problems, whereas ours is one of the combined source-channel coding.

We present four sufficient conditions and one necessary condition for the achievability of a distortion pair $\left(D_{1}, D_{2}\right)$. These conditions are expressed as a function of the channel signal-tonoise ratio (SNR) and of the source correlation.

Our contribution is in the following aspects:

- We suggest an extension for the Lapidoth-Tinguely vector-quantizer [1] to the case with unidirectional conferencing and derive the corresponding achievable rate-distortion region.

- We derive an achievable rate-distortion region when the capacity of the conference channel is unlimited.

- We derive a necessary condition for the achievability of a distortion pair $\left(D_{1}, D_{2}\right)$. This condition is obtained by some arguments reducing the multiple-access problem to a pointto-point problem. The key step therein is to upper-bound the maximal correlation between two simultaneous channel inputs, subject to conditional rate-distortion constraints, by using a result from maximum correlation theory.

- We derive the high-SNR asymptotics of an optimal scheme when the capacity of the conference channel is unlimited. In particular, we show that in this case a source-channel separation scheme is optimal.

- For a fixed conferencing capacity, high-SNR, and symmetric average-power constraints, we show that the latter source-channel separation scheme, which is optimal for unlimited conferencing capacity, is suboptimal compared to the vector-quantizer.

The paper is organized as follows. Section II presents our main results, while in Section III we prove the necessary condition. In Section IV and the Appendix we present our code construction and analyze its performance. The analysis for the rest of our main results appears in Sections VIX.

\section{MAin Results}

In this section we present one necessary condition and four sufficient conditions for the achievability of a distortion pair $\left(D_{1}, D_{2}\right)$; the sufficient conditions are stated in Theorem 2 , Corollary 1, and via the two source-channel separation schemes considered in Section II.C. The necessary condition also establishes the asymptotic behavior of an optimal scheme for a subset of distortion pairs, when the capacity of the conference channel is unlimited.

\section{A. Necessary condition for the achievability of $\left(D_{1}, D_{2}\right)$}

Theorem 1 A necessary condition for the achievability of a distortion pair $\left(D_{1}, D_{2}\right)$ over the Gaussian MAC with unidirectional conferencing is that for some $0 \leq \beta \leq 1$

$$
\begin{aligned}
R_{S_{1}, S_{2}}\left(D_{1}, D_{2}\right) & \leq \frac{1}{2} \log _{2}\left(1+\frac{P_{1}+P_{2}+2 \sqrt{\left(\rho^{2} \bar{\beta}+\beta\right)} \sqrt{P_{1} P_{2}}}{N}\right) \\
R_{S_{2} \mid S_{1}}\left(D_{2}\right) & \leq \frac{1}{2} \log _{2}\left(1+\frac{\bar{\beta} P_{2}\left(1-\rho^{2}\right)}{N}\right),
\end{aligned}
$$


where $R_{S_{1}, S_{2}}\left(D_{1}, D_{2}\right)$ denotes the rate-distortion function of a bivariate Gaussian source $\left\{\left(S_{1, k}, S_{2, k}\right)\right\}$, which is derived first in [7] and then in [1] Theorem III.1], and $R_{S_{2} \mid S_{1}}\left(D_{2}\right)$ denotes the rate-distortion function for $\left\{S_{2, k}\right\}$ when $\left\{S_{1, k}\right\}$ is given as side-information to both the encoder and the decoder.

\section{Proof: See Section ஹ.}

Remark 1 The necessary condition (9) is of the same flavor as the necessary condition in [1] Theorem IV.1]. Specifically, Condition (9) corresponds to the necessary and sufficient condition for the achievability of a distortion pair $\left(D_{1}, D_{2}\right)$ when the source $\left\{\left(S_{1, k}, S_{2, k}\right)\right\}$ is transmitted over a point to point additive white Gaussian noise (AWGN) channel of input power constraint $P_{1}+P_{2}+2 \sqrt{\left(\rho^{2} \bar{\beta}+\beta\right)} \sqrt{P_{1} P_{2}}$.

Remark 2 The necessary condition (9)-(10) is not a function of $C_{12}$. Therefore, we expect that it will be tight when the conferencing capacity is unlimited.

\section{B. Vector-quantizer scheme}

Our achievability result is based on an extension of the vector-quantizer scheme presented in [1], which benefits from the presence of the unidirectional conference channel. The encoding steps of our scheme are presented in Fig. 2

The source sequence $\mathbf{S}_{1}$ is quantized by Encoder 1 in two steps; first it is quantized by a rate- $R_{1}$ vector-quantizer where the quantized sequence is denoted by $\mathbf{U}_{1}^{*}$, then the quantization error of the first step is quantized by a rate- $R_{\mathrm{c}}$ vector-quantizer, where

$$
R_{\mathrm{c}}+1 / 2 \log \left(1-\rho^{2} 2^{-2 R_{1}}\left(1-2^{-2 R_{\mathrm{c}}}\right)\right) \leq C_{12},
$$

and the quantized sequence is denoted by $\mathbf{V}^{*}$. The source sequence $\mathbf{S}_{2}$ is quantized by Encoder 2 via a rate- $R_{2}$ vector-quantizer where the quantized sequence is denoted by $\mathbf{U}_{2}^{*}$. Encoder 1 informs Encoder 2 via the conference channel on the index of $\mathbf{V}^{*}$, taking into account that Encoder 2 has side-information $\mathbf{S}_{2}$, and consequently both encoders can cooperate in transmitting this sequence.

The channel input $\mathbf{X}_{1}$ is now given by

$$
\mathbf{X}_{1}=a_{1,1} \mathbf{U}_{1}^{*}+a_{1,2} \mathbf{V}^{*},
$$

where for $0 \leq \beta_{1} \leq 1$ the gains $a_{1,1}$ and $a_{1,2}$ are chosen as

$$
a_{1,1}=\sqrt{\frac{\bar{\beta}_{1} P_{1}}{\sigma^{2}\left(1-2^{-2 R_{1}}\right)}} \quad, \quad a_{1,2}=\sqrt{\frac{\beta_{1} P_{1}}{\sigma^{2} 2^{-2 R_{1}}\left(1-2^{-2 R_{\mathrm{c}}}\right)}} .
$$

This ensures that the input $\mathbf{X}_{1}$ satisfies the average-power constraint $P_{1}$. The channel input $\mathbf{X}_{2}$ is now given by

$$
\mathbf{X}_{2}=a_{2,1} \mathbf{U}_{2}^{*}+a_{2,2} \mathbf{V}^{*},
$$

where for $0 \leq \beta_{2} \leq 1$ and $\sigma_{v}^{2} \triangleq \sigma^{2} 2^{-2 R_{1}}\left(1-2^{-2 R_{\mathrm{c}}}\right)$, the gains $a_{2,1}$ and $a_{2,2}$ are chosen as

$$
\begin{aligned}
& a_{2,1}=\sqrt{\frac{\bar{\beta}_{2} P_{2}}{\sigma^{2}\left(1-2^{-2 R_{2}}\right)}}, \\
& a_{2,2}=\sqrt{\frac{P_{2}}{\sigma^{2}}}\left(\sqrt{\rho^{2} \bar{\beta}_{2}\left(1-2^{-2 R_{2}}\right)+\frac{\sigma^{2} \beta_{2}}{\sigma_{v}^{2}}}-\sqrt{\rho^{2} \bar{\beta}_{2}\left(1-2^{-2 R_{2}}\right)}\right) .
\end{aligned}
$$

This ensures that the input $\mathbf{X}_{2}$ satisfies the average-power constraint $P_{2}$. 


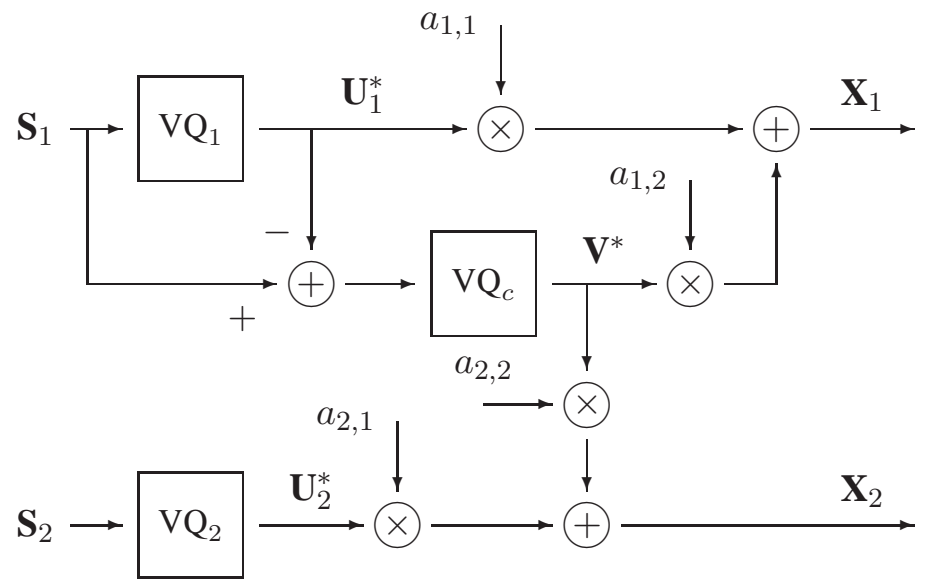

Fig. 2. The vector-quantizer flow

Based on the channel output $\mathbf{Y}$, the decoder first estimates the triplet $\left(\mathbf{U}_{1}^{*}, \mathbf{V}^{*}, \mathbf{U}_{2}^{*}\right)$ by performing joint decoding which takes into account the correlation between the sequences. The resulting decoded triplet is denoted by $\left(\hat{\mathbf{U}}_{1}, \hat{\mathbf{V}}, \hat{\mathbf{U}}_{2}\right)$. The decoder then treats $\left(\mathbf{S}_{1}, \mathbf{S}_{2}, \hat{\mathbf{U}} 1, \hat{\mathbf{V}}, \hat{\mathbf{U}}_{2}\right)$ as a jointly Gaussian tuple and forms its estimates of the source sequences $\mathbf{S}_{\nu}, \nu=1,2$ using minimum-mean-square-error (MMSE) estimates of $\mathbf{S}_{\nu}$ based on $\left(\hat{\mathbf{U}}_{1}, \hat{\mathbf{V}}, \hat{\mathbf{U}}_{2}\right)$, i.e.,

$$
\begin{aligned}
& \hat{\mathbf{S}}_{1}=\gamma_{1,1} \hat{\mathbf{U}}_{1}+\gamma_{1,2} \hat{\mathbf{U}}_{2}+\gamma_{1,3} \hat{\mathbf{V}} \approx \mathrm{E}\left[\mathbf{S}_{1} \mid \hat{\mathbf{U}}_{1}, \hat{\mathbf{V}}, \hat{\mathbf{U}}_{2}\right] \\
& \hat{\mathbf{S}}_{2}=\gamma_{2,1} \hat{\mathbf{U}}_{1}+\gamma_{2,2} \hat{\mathbf{U}}_{2}+\gamma_{2,3} \hat{\mathbf{V}} \approx \mathrm{E}\left[\mathbf{S}_{2} \mid \hat{\mathbf{U}}_{1}, \hat{\mathbf{V}}, \hat{\mathbf{U}}_{2}\right],
\end{aligned}
$$

where the approximate sign is due to the assumption that $\left(\mathbf{S}_{1}, \mathbf{S}_{2}, \hat{\mathbf{U}}_{1}, \hat{\mathbf{V}}, \hat{\mathbf{U}}_{2}\right)$ are jointly Gaussian. Here

$$
\begin{aligned}
\gamma_{1,1}=\gamma_{1,3} & =\frac{1-\rho^{2}\left(1-2^{-2 R_{2}}\right)}{1-\rho^{2}\left(1-2^{-2 R_{2}}\right)\left(1-2^{-2\left(R_{1}+R_{\mathrm{c}}\right)}\right)} \\
\gamma_{1,2} & =\frac{\rho 2^{-2\left(R_{1}+R_{\mathrm{c}}\right)}}{1-\rho^{2}\left(1-2^{-2 R_{2}}\right)\left(1-2^{-2\left(R_{1}+R_{\mathrm{c}}\right)}\right)} \\
\gamma_{2,1}=\gamma_{2,3} & =\frac{\rho 2^{-2 R_{2}}}{1-\rho^{2}\left(1-2^{-2 R_{2}}\right)\left(1-2^{-2\left(R_{1}+R_{\mathrm{c}}\right)}\right)} \\
\gamma_{2,2} & =\frac{1-\rho^{2}\left(1-2^{-2\left(R_{1}+R_{\mathrm{c}}\right)}\right)}{1-\rho^{2}\left(1-2^{-2 R_{2}}\right)\left(1-2^{-2\left(R_{1}+R_{\mathrm{c}}\right)}\right)}
\end{aligned}
$$

are the coefficients of the linear MMSE estimators of $\mathbf{S}_{\nu}$ given $\left(\hat{\mathbf{U}}_{1}, \hat{\mathbf{V}}, \hat{\mathbf{U}}_{2}\right)$. In Lemma 11 (in the Appendix) we prove that

$$
0<\gamma_{1,1}, \gamma_{1,3}, \gamma_{2,2} \leq 1 \quad \text { and } \quad 0<\gamma_{1,2}, \gamma_{2,1}, \gamma_{2,3} \leq \rho .
$$

A detailed description of the scheme is given in Section IV

The distortion pairs achieved by this vector-quantizer (VQ) scheme are described in the next theorem.

Theorem 2 The distortions achieved by the vector-quantizer scheme are all pairs $\left(D_{1}, D_{2}\right)$ satisfying

$$
D_{1}>\sigma^{2} 2^{-2\left(R_{1}+R_{\mathrm{c}}\right)} \frac{1-\rho^{2}\left(1-2^{-2 R_{2}}\right)}{1-\rho^{2}\left(1-2^{-2 R_{2}}\right)\left(1-2^{-2\left(R_{1}+R_{\mathrm{c}}\right)}\right)}
$$




$$
D_{2}>\sigma^{2} 2^{-2 R_{2}} \frac{1-\rho^{2}\left(1-2^{-2\left(R_{1}+R_{\mathrm{c}}\right)}\right)}{1-\rho^{2}\left(1-2^{-2 R_{2}}\right)\left(1-2^{-2\left(R_{1}+R_{\mathrm{c}}\right)}\right)}
$$

where, for some $0 \leq \beta_{1}, \beta_{2} \leq 1$, the rate-triple $\left(R_{1}, R_{2}, R_{\mathrm{c}}\right)$ satisfies

$$
\begin{aligned}
R_{1} & <\frac{1}{2} \log \left(\frac{\bar{\beta}_{1} P_{1}\left(1-\tilde{\rho}^{2}-\bar{\rho}^{2}\right)+N\left(1-\bar{\rho}^{2}\right)}{N\left(1-\tilde{\rho}^{2}-\bar{\rho}^{2}\right)}\right) \\
R_{2} & <\frac{1}{2} \log \left(\frac{\bar{\beta}_{2} P_{2}\left(1-\tilde{\rho}^{2}-\bar{\rho}^{2}\right)+N}{N\left(1-\tilde{\rho}^{2}-\bar{\rho}^{2}\right)+\lambda_{2}}\right) \\
R_{\mathrm{c}} & <\frac{1}{2} \log \left(\frac{\eta^{2}\left(1-\tilde{\rho}^{2}-\bar{\rho}^{2}\right)+N\left(1-\tilde{\rho}^{2}\right)}{N\left(1-\tilde{\rho}^{2}-\bar{\rho}^{2}\right)+\lambda_{c}}\right) \\
R_{1}+R_{2} & <\frac{1}{2} \log \left(\frac{\lambda_{12}-\bar{\beta}_{2} P_{2} \bar{\rho}^{2}+N}{\left(1-\bar{\beta}_{2} P_{2} \bar{\rho}^{2} \lambda_{12}^{-1}\right) N\left(1-\tilde{\rho}^{2}\right)}\right) \\
R_{1}+R_{\mathrm{c}} & <\frac{1}{2} \log \left(\frac{\left(\lambda_{1 c}+N\right)\left(\bar{\beta}_{1} P_{1}+\eta^{2}\right)}{\lambda_{1 c} N}\right) \\
R_{2}+R_{\mathrm{c}} & <\frac{1}{2} \log \left(\frac{\lambda_{2 c}-\bar{\beta}_{2} P_{2} \tilde{\rho}^{2}+N}{\left(1-\bar{\beta}_{2} P_{2} \tilde{\rho}^{2} \lambda_{2 c}^{-1}\right) N\left(1-\bar{\rho}^{2}\right)}\right) \\
R_{1}+R_{2}+R_{\mathrm{c}} & <\frac{1}{2} \log \left(\frac{\lambda_{12}+2 \eta \bar{\rho} \sqrt{\bar{\beta}_{2} P_{2}}+\eta^{2}+N}{N\left(1-\tilde{\rho}^{2}\right)\left(1-\bar{\rho}^{2}\right)}\right) \\
C_{12} & >R_{\mathrm{c}}+\frac{1}{2} \log \left(1-\rho^{2} 2^{-2 R_{1}}\left(1-2^{-2 R_{\mathrm{c}}}\right)\right)
\end{aligned}
$$

and where

$$
\begin{aligned}
\tilde{\rho} & \triangleq \rho \sqrt{\left(1-2^{-2 R_{1}}\right)\left(1-2^{-2 R_{2}}\right)} \\
\bar{\rho} & \triangleq \rho \sqrt{2^{-2 R_{1}}\left(1-2^{-2 R_{2}}\right)\left(1-2^{-2 R_{c}}\right)} \\
\lambda_{2} & \triangleq \frac{N^{2} \bar{\rho}^{2} \tilde{\rho}^{2}\left(2+\tilde{\rho}^{2}\right)}{\beta_{2} P_{2}\left(1-\tilde{\rho}^{2}-\bar{\rho}^{2}\right)+N} \\
\eta & \triangleq \sigma_{v}\left(\sqrt{\beta_{1} P_{1}} \sigma_{v}^{-1}+a_{2,2}\right) \\
\lambda_{c} & \triangleq \frac{N^{2} \bar{\rho}^{2}\left(\bar{\rho}^{2} \bar{\beta}_{1} P_{1}-\tilde{\rho}^{2} \sigma_{v}^{2}\right)}{\sigma_{v}^{2}\left(\eta^{2}\left(1-\tilde{\rho}^{2}-\bar{\rho}^{2}\right)+N\left(1-\tilde{\rho}^{2}\right)\right)} \\
\lambda_{12} & \triangleq \bar{\beta}_{1} P_{1}+2 \tilde{\rho} \sqrt{\bar{\beta}_{1} \bar{\beta}_{2} P_{1} P_{2}}+\bar{\beta}_{2} P_{2} \\
\lambda_{1 c} & \triangleq \bar{\beta}_{1} P_{1}(1-\tilde{\rho})+\eta^{2}\left(1-\bar{\rho}^{2}\right)-2 \eta \sigma_{v}^{-1} \bar{\rho}^{2} \sqrt{\bar{\beta}_{1} P_{1} \sigma^{2}\left(1-2^{-2 R_{1}}\right)} \\
\lambda_{2 c} & \triangleq \bar{\beta}_{2} P_{2}+2 \eta \bar{\rho} \sqrt{\bar{\beta}_{2} P_{2}}+\eta^{2} .
\end{aligned}
$$

Proof: See Section IV

Remark 3 The substitution of $C_{12}=0$ in Theorem 2 (which then implies $R_{\mathrm{c}}=0$, as well as $\bar{\beta}_{1}=\bar{\beta}_{2}=1$ as per (12) and (13) based on the code-construction in Section $\square A$ ) recovers the Lapidoth-Tinguely achievable rate-distortion region [1] Theorem IV.4].

Based on Theorem 2 we now present sufficient conditions for the achievability of $\left(D_{1}, D_{2}\right)$ when $C_{12}=\infty$.

Corollary 1 When $C_{12}$ is unlimited, the distortions achieved by the vector-quantizer scheme 


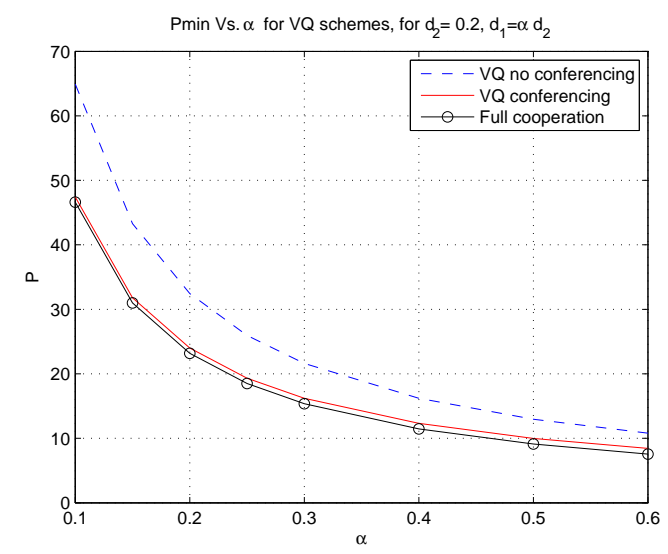

Fig. 3. $\quad P_{\min }$ for VQ when $d_{2}=0.2, d_{1}=\alpha d_{2}, \rho=0.5, N=1$

are all pairs $\left(D_{1}, D_{2}\right)$ satisfying

$$
\begin{aligned}
& D_{1}>\sigma^{2} 2^{-2 R_{\mathrm{c}}} \frac{1-\rho^{2}\left(1-2^{-2 R_{2}}\right)}{1-\hat{\rho}^{2}} \\
& D_{2}>\sigma^{2} 2^{-2 R_{2}} \frac{1-\rho^{2}\left(1-2^{-2 R_{\mathrm{c}}}\right)}{1-\hat{\rho}^{2}}
\end{aligned}
$$

where, for some $0 \leq \beta \leq 1$, the rate-pair $\left(R_{2}, R_{\mathrm{c}}\right)$ satisfies

$$
\begin{aligned}
R_{2} & <\frac{1}{2} \log \left(\frac{\bar{\beta} P_{2}\left(1-\hat{\rho}^{2}\right)+N}{N\left(1-\hat{\rho}^{2}\right)}\right) \\
R_{\mathrm{c}} & <\frac{1}{2} \log \left(\frac{\delta_{1}^{2}\left(1-\hat{\rho}^{2}\right)+N}{N\left(1-\hat{\rho}^{2}\right)}\right) \\
R_{2}+R_{\mathrm{c}} & <\frac{1}{2} \log \left(\frac{\delta_{2}+N}{N\left(1-\hat{\rho}^{2}\right)}\right),
\end{aligned}
$$

and where

$$
\begin{aligned}
& \hat{\rho} \triangleq \rho \sqrt{\left(1-2^{-2 R_{2}}\right)\left(1-2^{-2 R_{\mathrm{c}}}\right)} \\
& \delta_{1} \triangleq \sqrt{P_{1}}+\sqrt{P_{2}}\left(\sqrt{\bar{\beta} \hat{\rho}^{2}+\beta}-\sqrt{\bar{\beta} \hat{\rho}^{2}}\right) \\
& \delta_{2} \triangleq P_{1}+P_{2}+2 \sqrt{\left(\bar{\beta} \hat{\rho}^{2}+\beta\right) P_{1} P_{2}} .
\end{aligned}
$$

Remark 4 For the achievability of the distortion pairs in Corollary $\square$ it suffices that $R_{\mathrm{c}}+$ $\frac{1}{2} \log \left(1-\rho^{2}\left(1-2^{-2 R_{\mathrm{c}}}\right)\right) \leq C_{12}$.

To demonstrate the benefit of conferencing for the VQ scheme we compare the performance of the VQ with unlimited conferencing capacity to the performance of the VQ without conferencing (i.e. the VQ in the Lapidoth-Tinguely MAC model). We fix $d_{2}$ and let $d_{1}=\alpha d_{2}$ and assume that the encoders are subject to symmetric average-power constraints. Fig. 3 compares the required average-power for the VQ with unlimited conferencing capacity and without conferencing, for attaining a desired distortion pair $\left(\alpha d_{2}, d_{2}\right)$. The figure displays also the minimum required power for attaining the desired distortions when $\left(\mathbf{S}_{1}, \mathbf{S}_{2}\right)$ is available at both encoders hence they can fully cooperate in the source description and therefore $R_{\mathbf{S}_{1}, \mathbf{S}_{2}}\left(\alpha d_{2}, d_{2}\right)=\frac{1}{2} \log \left(1+\frac{4 P}{N}\right)$. 


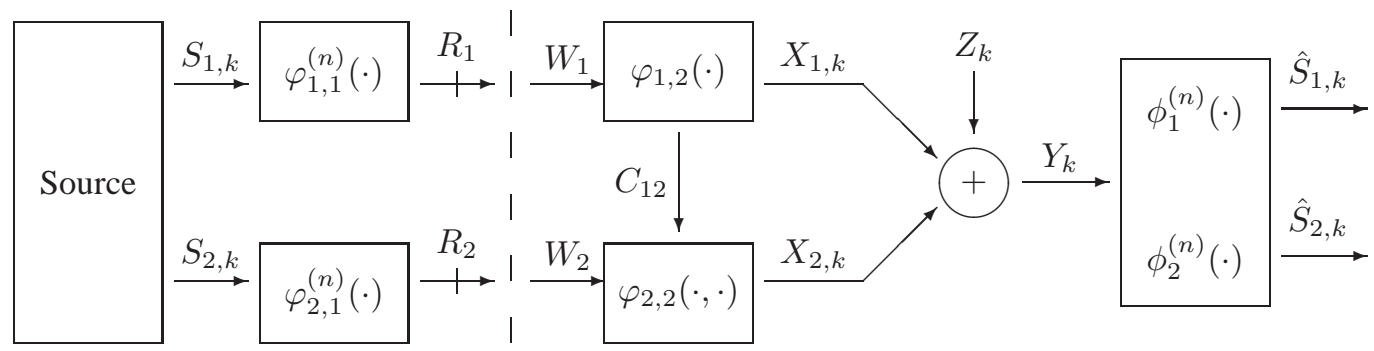

Fig. 4. Separation scheme 1; Gaussian two-encoder source-coding combined with Gaussian MAC with unidirectional conferencing channel-coding

\section{Source-Channel Separation}

Next, we compare the performance of our vector-quantizer scheme with the performance of two optional source-channel separation schemes, for the case of unlimited conferencing capacity.

1) Source-Channel Separation Scheme 1: We consider the set of distortion pairs that are achieved by combining the optimal scheme for the source-coding problem without conferencing with the optimal scheme for the channel-coding problem with unidirectional conferencing, as shown in Fig. 4

The rate-distortion region associated with the source-coding problem can be found in [13], [14] and is described as follows.

Proposition 1 [13], [14] A distortion pair $\left(d_{1}, d_{2}\right)$ is achievable for the Gaussian two-terminal source-coding problem if, and only if, $\left(R_{1}, R_{2}\right) \in \mathcal{R}\left(d_{1}, d_{2}\right)$ where

$$
\begin{aligned}
& \mathcal{R}\left(d_{1}, d_{2}\right)=\left\{\left(R_{1}, R_{2}\right):\right. R_{1} \\
& \geq \frac{1}{2} \log _{2}^{+}\left[\frac{1-\rho^{2}\left(1-2^{-2 R_{2}}\right)}{d_{1}}\right] \\
& R_{2} \geq \frac{1}{2} \log _{2}^{+}\left[\frac{1-\rho^{2}\left(1-2^{-2 R_{1}}\right)}{d_{2}}\right] \\
& R_{1}+R_{2}\left.\geq \frac{1}{2} \log _{2}^{+}\left[\frac{\left(1-\rho^{2}\right) \gamma\left(d_{1}, d_{2}\right)}{2 d_{1} d_{2}}\right]\right\},
\end{aligned}
$$

with $\gamma\left(d_{1}, d_{2}\right)=1+\sqrt{1+\frac{4 \rho^{2} d_{1} d_{2}}{\left(1-\rho^{2}\right)^{2}}}$ and $\log _{2}^{+}[x]=\max \left\{0, \log _{2}(x)\right\}$.

The distortion pairs achievable by source-channel separation follow now by combining the latter set of rate pairs with the capacity region of the Gaussian MAC with unidirectional conference link reported in [10], which for $C_{12}=\infty$, is expressed by

$$
\begin{aligned}
\mathcal{C}=\bigcup_{0 \leq \beta \leq 1}\left\{\left(R_{1}, R_{2}\right): \quad R_{2}\right. & \leq \frac{1}{2} \log _{2}\left(1+\bar{\beta} P_{2} / N\right) \\
R_{1}+R_{2} & \left.\leq \frac{1}{2} \log _{2}\left(1+\left(P_{1}+P_{2}+2 \sqrt{\beta P_{1} P_{2}}\right) / N\right)\right\} .
\end{aligned}
$$

Note that, by [5, Theorem 1], when $C_{12}=\infty$ source-channel separation is optimal for lossless transmission of both sources and by [6] source-channel separation is optimal also when $d_{1}=0$ and $d_{2}>0$.

Next, we compare the performance of the vector-quantizer scheme, with that of sourcechannel separation scheme 1 , for lossy trasmission. We fix $d_{2}=0.2$ and let $d_{1}=\alpha d_{2}$. In 


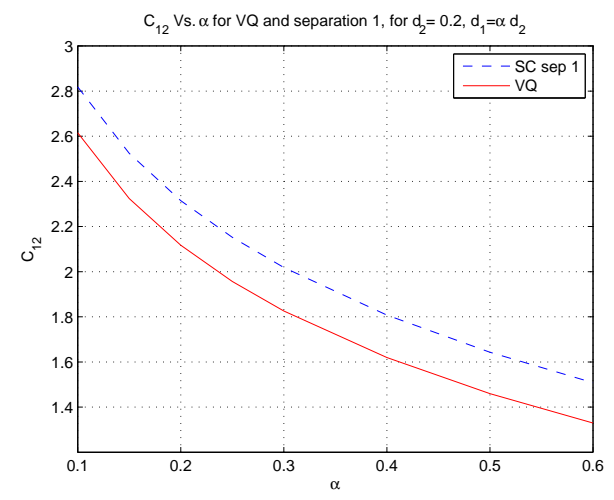

Fig. 5. $C_{12}$ for VQ and SC sep 1 when $d_{2}=0.2, d_{1}=\alpha d_{2}, \rho=0.5, N=1$

addition, we assume that the encoders are subject to symmetric average-power constraints. Fig. 5 shows the required conferencing capacity for the VQ and for separation scheme 1, for attaining a desired distortion pair $\left(\alpha d_{2}, d_{2}\right)$ (The figure uses the shorthand notation SC for source-channel). While both schemes require the same average-power, the VQ requires a smaller conferencing capacity.

For the set of distortion pairs $\left(d_{1}<1, d_{2}=1\right)$ we can show analytically that the VQ scheme outperforms separation scheme 1 in the required conferencing rate.

For separation scheme 1 , by choosing $R_{2}=0$ we obtain the following bounds on $R_{1}$,

- Source coding: $R_{1} \geq \frac{1}{2} \log \frac{1}{d_{1}}$.

- Channel coding: $R_{1} \leq \frac{1}{2} \log \left(1+\frac{4 P}{N}\right)$,

where $R_{1} \leq C_{12}$.

On the other hand, for the VQ scheme by choosing $R_{2}=0$ we obtain the following bounds on $R_{\mathrm{c}}$ (which plays the role of $R_{1}$ in separation scheme 1 ),

- $R_{\mathrm{c}} \geq \frac{1}{2} \log \frac{1}{d_{1}}$.

- $R_{\mathrm{c}} \leq \frac{1}{2} \log \left(1+\frac{4 P}{N}\right)$,

where $R_{\mathrm{c}}+1 / 2 \log \left[1-\rho^{2}\left(1-2^{-2 R_{\mathrm{c}}}\right)\right] \leq C_{12}$.

Moreover, in this special case, the $C_{12}$ versus $\left(P, d_{1}\right)$ tradeoff of the VQ is optimal as can be argued as follows:

- Over a point-to-point channel with average power $4 P$ quantizing the source at the channel capacity rate attains the minimal distortion $d_{1}=\frac{N}{4 P+N}$.

- The Wyner-Ziv (WZ) [8] rate for the Gaussian WZ problem, coincides with our lower bound on $C_{12}$ :

$$
\begin{aligned}
R_{\mathrm{WZ}}\left(d_{1}\right) & =1 / 2 \log \left[\left(1-\rho^{2}\right) / d_{1}+\rho^{2}\right] \\
& =R_{\mathrm{c}}+1 / 2 \log \left[1-\rho^{2}\left(1-2^{-2 R_{\mathrm{c}}}\right)\right] .
\end{aligned}
$$

2) Source-Channel Separation Scheme 2: We consider next the set of distortion pairs that are achieved by combining an achievable rate-distortion scheme for the source-coding problem with unidirectional conference link, with the optimal scheme for the channel-coding problem without conferencing, as shown in Fig. 6, An achievable rate-distortion region for the sourcecoding problem with unidirectional conference link can be found in [9, Theorem 5.1] (for the open switch problem) and is described as follows. Let $\mathcal{P}\left(D_{1}, D_{2}\right)$ be the set of all triples of random variables $(U, V, W)$ jointly distributed with $\left(S_{1}, S_{2}\right)$ such that

1) $U \multimap\left(S_{2}, W\right) \multimap\left(S_{1}, W\right) \multimap V$ and $W \multimap S_{1} \multimap S_{2}$ are Markov chains, 


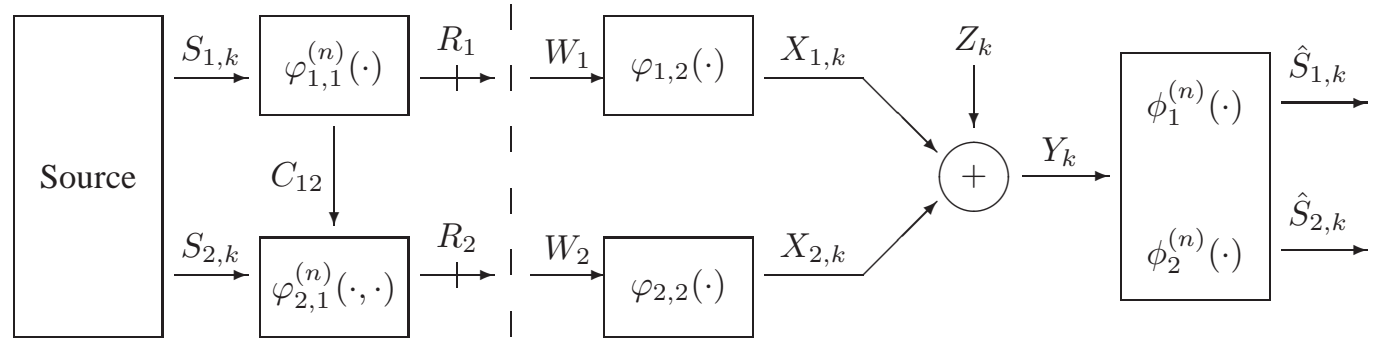

Fig. 6. Separation scheme 2; Gaussian two-encoder with unidirectional conferencing source-coding combined with Gaussian MAC channel-coding

2) $\sigma_{S_{1} \mid U, V, W}^{2} \leq D_{1}, \sigma_{S_{2} \mid U, V, W}^{2} \leq D_{2}$.

Furthermore, define

$$
\begin{aligned}
& \mathcal{R}^{(\text {in })}\left(D_{1}, D_{2}\right)=\bigcup_{(U, V, W) \in \mathcal{P}\left(D_{1}, D_{2}\right)}\left\{\left(R_{1}, R_{2}\right):\right. C_{12} \geq I\left(S_{1} ; W \mid S_{2}\right) \\
& R_{1} \geq I\left(S_{1} ; V \mid U, W\right) \\
& R_{2} \geq I\left(S_{2} ; U \mid V, W\right) \\
&\left.R_{1}+R_{2} \geq I\left(S_{1}, S_{2} ; U, V, W\right)\right\} .
\end{aligned}
$$

Proposition 2 [9 Theorem 5.1] $\mathcal{R}^{(\mathrm{in})}\left(D_{1}, D_{2}\right)$ is contained within the rate-distortion region $\mathcal{R}\left(D_{1}, D_{2}\right)$ for source-coding of correlated sources with unidirectional conference link of capacity $C_{12}$. The inner bound is tight when $S_{1}$ is reconstructed almost perfectly.

The Gaussian achievable rate-distortion region associated with $\mathcal{R}^{(\mathrm{in})}\left(D_{1}, D_{2}\right)$ is characterized as follows.

Proposition 3 For a nonnegative pair $\left(D_{1}, D_{2}\right)$, the rate-distortion region $\mathcal{R}\left(D_{1}, D_{2}\right)$ contains the region $\mathcal{R}_{\mathrm{G}}\left(D_{1}, D_{2}\right)$ defined by

$$
\begin{aligned}
\mathcal{R}_{\mathrm{G}}\left(D_{1}, D_{2}\right)=\bigcup_{\sigma_{w}^{2}, \sigma_{u}^{2}, \sigma_{v}^{2}}\left\{\left(R_{1}, R_{2}\right): \quad C_{12}\right. & \geq \frac{1}{2} \log _{2}\left[1+\frac{\sigma^{2}\left(1-\rho^{2}\right)}{\sigma_{w}^{2}}\right] \\
R_{1} & \geq \frac{1}{2} \log _{2}\left[1+\frac{\sigma^{2}}{\sigma_{v}^{2}} \frac{\left(1-\rho^{2}\right)+\frac{\sigma_{u}^{2}}{\sigma^{2}}}{\left.1+\frac{\sigma^{2}\left(1-\rho^{2}\right)}{\sigma_{w}^{2}}+\left(\frac{\sigma_{u}^{2}}{\sigma_{w}^{2}}+\frac{\sigma_{u}^{2}}{\sigma^{2}}\right)\right]}\right. \\
R_{2} & \geq \frac{1}{2} \log _{2}\left[1+\frac{\sigma^{2}}{\sigma_{u}^{2}} \frac{1+\left(\frac{\sigma^{2}}{\sigma_{w}^{2}}+\frac{\sigma^{2}}{\sigma_{v}^{2}}\right)\left(1-\rho^{2}\right)}{1+\left(\frac{\sigma^{2}}{\sigma_{w}^{2}}+\frac{\sigma^{2}}{\sigma_{v}^{2}}\right)}\right] \\
R_{1}+R_{2} & \geq \frac{1}{2} \log _{2}[\Delta] \\
D_{1} & \leq \sigma^{2} \frac{1+\frac{\sigma^{2}}{\sigma_{u}^{2}}\left(1-\rho^{2}\right)}{\Delta} \\
D_{2} & \left.\leq \sigma^{2} \frac{1+\sigma^{2}\left(1-\rho^{2}\right)\left(\frac{1}{\sigma_{v}^{2}}+\frac{1}{\sigma_{w}^{2}}\right)}{\Delta}\right\},
\end{aligned}
$$

with $\Delta=1+\frac{\sigma^{2}}{\sigma_{u}^{2}}+\sigma^{2}\left(1+\frac{\sigma^{2}}{\sigma_{u}^{2}}\left(1-\rho^{2}\right)\right)\left(\frac{1}{\sigma_{v}^{2}}+\frac{1}{\sigma_{w}^{2}}\right)$. 


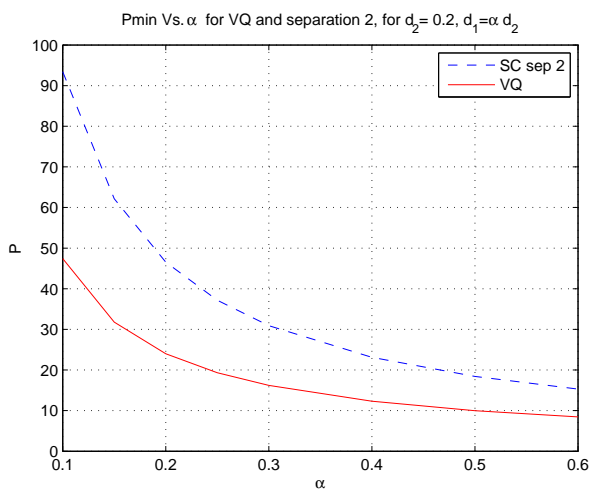

Fig. 7. $P_{\min }$ for VQ and SC sep 2 when $d_{2}=0.2, d_{1}=\alpha d_{2}, \rho=0.5, N=1$

\section{Proof: See Section V}

The distortion pairs achievable by this source-channel separation scheme follow now by combining the latter set of rate pairs with the capacity region of the Gaussian MAC without unidirectional conference link, which is expressed by

$$
\begin{aligned}
\mathcal{C}_{\mathrm{G}}\left(P_{1}, P_{2}\right)=\left\{\left(R_{1}, R_{2}\right): \quad\right. & \leq \frac{1}{2} \log _{2}\left(1+\frac{P_{1}}{N}\right) \\
R_{2} & \leq \frac{1}{2} \log _{2}\left(1+\frac{P_{2}}{N}\right) \\
R_{1}+R_{2} & \left.\leq \frac{1}{2} \log _{2}\left(1+\frac{P_{1}+P_{2}}{N}\right)\right\} .
\end{aligned}
$$

We compare the performance of the source-channel separation scheme 2 inner bound with that of the vector-quantizer, for unlimited conferencing capacity. We fix $d_{2}=0.2$ and let $d_{1}=\alpha d_{2}$. In addition, we assume that the encoders are subject to symmetric average-power constraints. Fig. 7 compares the required average-power for attaining a desired distortion pair $\left(\alpha d_{2}, d_{2}\right)$. We see that the VQ scheme requires less average-power than source-channel separation scheme 2 while both schemes require the same conferencing capacity.

\section{High-SNR asymptotics with unlimited conferencing capacity}

We consider next the high-SNR asymptotics of an optimal scheme when the conferencing capacity is unlimited. To this end, let $\left(d_{1}^{*}, d_{2}^{*}\right)$ denote an arbitrary normalized distortion pair resulting from an optimal scheme. For a subset of those distortion pairs -i.e. distortion pairs satisfying $d_{1} d_{2}=O\left(\frac{N}{P_{1}+P_{2}}\right)$ where $\frac{N}{P_{1}+P_{2}} \ll 1$, the high-SNR behavior is described in the following theorem.

Theorem 3 The high-SNR asymptotics for the Gaussian MAC with unlimited $C_{12}$ satisfies

$$
\lim _{N \rightarrow 0} \frac{P_{1}+P_{2}+2 \varrho_{\infty}^{*} \sqrt{P_{1} P_{2}}}{N} d_{1}^{*} d_{2}^{*}=\left(1-\rho^{2}\right),
$$

provided that $d_{1}^{*} \leq 1, d_{2}^{*} \leq 1$, and that

$$
\lim _{N \rightarrow 0} \frac{N}{d_{1}^{*} P_{1}}=0, \quad \text { and } \quad \lim _{N \rightarrow 0} \frac{N}{d_{2}^{*} P_{2}}=0,
$$


where

$$
\varrho_{\infty}^{*}=\sqrt{1-\frac{N\left(1-\rho^{2}\right)}{d_{2}^{*} P_{2}}} .
$$

Proof: See Section VI

Corollary 2 The high-SNR asymptotics for separation scheme 1 for the Gaussian MAC with unlimited $C_{12}$ satisfies

$$
\lim _{N \rightarrow 0} \frac{P_{1}+P_{2}+2 \varrho_{\mathrm{sep} 1}^{*} \sqrt{P_{1} P_{2}}}{N} d_{1} d_{2} \geq\left(1-\rho^{2}\right),
$$

provided that $d_{1} \leq 1, d_{2} \leq 1$, and that

$$
\lim _{N \rightarrow 0} \frac{N}{d_{1} P_{1}}=0, \quad \text { and } \quad \lim _{N \rightarrow 0} \frac{N}{d_{2} P_{2}}=0,
$$

where

$$
\varrho_{\text {sep } 1}^{*}=\sqrt{1-\frac{N\left(1-\rho^{2}\right)}{d_{2} P_{2}}} .
$$

Proof: See Section VIII

We conclude with the following extension to [6] which asserts that:

Corollary 3 For high-SNR with $\rho>0, C_{12}=\infty$, and $\left(d_{1}, d_{2}\right)$ such that $\lim _{N \rightarrow 0} \frac{N}{d_{1} P_{1}}=0$, and $\lim _{N \rightarrow 0} \frac{N}{d_{2} P_{2}}=0$, source-channel separation scheme 1 is optimal in the sense of attaining the optimal $d_{1} d_{2}$ given the system parameters $\left(\rho, P_{1}, P_{2}, N\right)$.

We restate Theorem 3 more specifically for the "semi-symmetric" case where $P_{1}=P_{2}=P$ while $\left(d_{1}, d_{2}\right)$ satisfies (27).

Corollary 4 In the "semi-symmetric" case, when $\left(d_{1}, d_{2}\right)$ satisfies (27)

$$
\begin{aligned}
\lim _{P / N \gg 1} d_{1} d_{2} & =\frac{N}{2 P} \cdot \frac{1-\rho^{2}}{1+\sqrt{1-\frac{N\left(1-\rho^{2}\right)}{d_{2} P}}} \\
& \approx \frac{N}{2 P} \cdot \frac{1-\rho^{2}}{2-\frac{N\left(1-\rho^{2}\right)}{2 d_{2} P}} .
\end{aligned}
$$

Discussion: The asymptotic correlation can be explained as follows. Separation scheme 1, when generating the channel inputs $\left(\mathbf{X}_{1}, \mathbf{X}_{2}\right)$, ignores the source correlation and transmits two independent messages via Willems's code construction for the MAC with conferencing [12]. As a result, the correlation between the channel inputs is $\sqrt{\beta}$, where $\beta$ is the fraction of power that Encoder 2 transmits in coherence with Encoder 1. In contrast, the vector-quantizer does exploit the source correlation and exhibits additional gain due to the correlation between $\mathbf{V}^{*}$ and $\mathbf{U}_{2}^{*}$ which is reflected by the larger correlation coefficient $\sqrt{\rho^{2} \bar{\beta}+\beta}$. Nevertheless, when $C_{12}=\infty$, the final maximization of both correlation expressions, each over its admissible domain of $\beta$, yields an identical result. This is explained by the fact that the asymptotic product 
(29) is attained via the separation scheme by the rate pair

$$
\begin{aligned}
R_{1} & =\frac{1}{2} \log \frac{2 d_{2} P\left(1+\sqrt{1-\frac{N\left(1-\rho^{2}\right)}{d_{2} P}}\right)}{N\left(1-\rho^{2}\right)} \\
R_{2} & =\frac{1}{2} \log \frac{1-\rho^{2}}{d_{2}},
\end{aligned}
$$

and by the vector-quantizer via the rate pair

$$
\begin{aligned}
R_{\mathrm{c}} & =\frac{1}{2} \log \frac{2 d_{2} P\left(1+\sqrt{1-\frac{N\left(1-\rho^{2}\right)}{d_{2} P}}\right)}{N\left(1-\rho^{2}\right)} \\
R_{2} & =\frac{1}{2} \log \frac{1}{d_{2}} .
\end{aligned}
$$

Consequently, the separation strategy which exploits the source correlation at the source-coding part sends at a lower $R_{2}$ rate which in turn increases the admissible domain of $\beta$. For separation scheme $1, C_{12}=R_{1}$, while for the vector-quantizer $C_{12}=R_{\mathrm{c}}+\frac{1}{2} \log \left(1-\rho^{2}\right)$.

Next, let us compare this asymptotic behavior to the asymptotic behavior of the vectorquantizer without conferencing, when $\left(d_{1}, d_{2}\right)$ satisfies [27), as reported in [1, Section IV.D]

$$
\lim _{P / N \gg 1} d_{1} d_{2} \approx \frac{N}{2 P} \cdot \frac{1-\rho^{2}}{1+\rho} .
$$

As noted in [1], the gain $1-\rho^{2}$ in the numerator on the r.h.s. of (30) is due to the fact that the receiver exploits the source correlation in joint-typicality decoding, while the gain $1+\rho$ in the denominator is due to the correlation $\rho$ that the encoders build on the channel inputs $\left(\mathbf{X}_{1}, \mathbf{X}_{2}\right)$. The asymptotic expression (29) demonstrates that with unlimited unidirectional conferencing capacity, both the vector quantizer and separation scheme 1, exploit the source correlationeach in its own way - and increase the correlation on the channel inputs to $2-\frac{N\left(1-\rho^{2}\right)}{2 d_{2} P} \rightarrow 2$ for $\frac{N}{d_{2} P} \ll 1$.

\section{E. High-SNR asymptotics with fixed conferencing capacity}

We consider first the high-SNR asymptotics of source-channel separation scheme 1 when the conferencing capacity $C_{12}$ is fixed.

Corollary 5 The high-SNR asymptotics for separation scheme 1 for the Gaussian MAC with fixed unidirectional conferencing capacity $C_{12}=C$ satisfies

$$
\lim _{N \rightarrow 0} d_{1} d_{2} \geq \frac{N\left(1-\rho^{2}\right)}{P_{1}+P_{2}+2 \varrho_{\text {sep } 1}^{*} \sqrt{P_{1} P_{2}}}
$$

provided that $d_{1} \leq 1, d_{2} \leq 1$ and that $\left(d_{1}, d_{2}\right)$ satisfy (27), where

$$
\varrho_{\mathrm{sep} 1}^{*}=\sqrt{1-\frac{N\left(1-\rho^{2}\right)}{d_{1} P_{1}} 2^{-2 C}} \sqrt{1-\frac{N\left(1-\rho^{2}\right)}{d_{2} P_{2}}} .
$$

Proof: See Section VIII

We consider next the high-SNR asymptotics of the vector-quantizer scheme when the conferencing capacity $C_{12}$ is fixed. 
Corollary 6 The high-SNR asymptotics for the vector-quantizer scheme for the Gaussian MAC with fixed unidirectional conferencing capacity $C_{12}=C$ satisfies

$$
\lim _{N \rightarrow 0} d_{1} d_{2} \geq \frac{N\left(1-\rho^{2}\right)\left(1-\check{\rho}^{2}\right)}{P_{1}+P_{2}+2 \varrho_{\mathrm{VQ}}^{*} \sqrt{P_{1} P_{2}}}
$$

provided that $d_{1} \leq 1, d_{2} \leq 1$, and that $\left(d_{1}, d_{2}\right)$ satisfy (27), where

$$
\begin{aligned}
\check{\rho} & \triangleq \rho \sqrt{2^{-2 R_{1}}\left(1-2^{-2 R_{\mathrm{c}}}\right)} \\
R_{\mathrm{c}} & \leq C_{12}-1 / 2 \log \left(1-\check{\rho}^{2}\right),
\end{aligned}
$$

and

$$
\varrho_{\mathrm{VQ}}^{*} \geq \rho 2^{-C} \sqrt{\frac{N}{d_{1} P_{1}}} \sqrt{\frac{N}{d_{2} P_{2}}}+\sqrt{1-\frac{N}{d_{1} P_{1}} 2^{-2 C}} \sqrt{1-\frac{N}{d_{2} P_{2}}} .
$$

Proof: See Section IX

Next, we compare the maximum correlation that can be achieved by the two schemes when $P_{1}=P_{2}=P$. For separation scheme 1 we obtain

$$
\begin{aligned}
\varrho_{\mathrm{sep} 1}^{*} & =\sqrt{1-\frac{N\left(1-\rho^{2}\right)}{d_{1} P} 2^{-2 C}} \sqrt{1-\frac{N\left(1-\rho^{2}\right)}{d_{2} P}} \\
& \approx\left[1-\frac{N\left(1-\rho^{2}\right)}{2 d_{1} P} 2^{-2 C}\right]\left[1-\frac{N\left(1-\rho^{2}\right)}{2 d_{2} P}\right] \\
& \approx 1-\frac{N}{2 P}\left(\frac{2^{-2 C}}{d_{1}}+\frac{1}{d_{2}}\right)\left(1-\rho^{2}\right),
\end{aligned}
$$

where in both approximation steps we use that $\frac{N}{d_{\nu} P} \ll 1, \nu=1,2$.

For the vector-quantizer we obtain

$$
\begin{aligned}
\varrho_{\mathrm{VQ}}^{*} & \geq \rho 2^{-C} \sqrt{\frac{N}{d_{1} P}} \sqrt{\frac{N}{d_{2} P}}+\sqrt{1-\frac{N}{d_{1} P} 2^{-2 C}} \sqrt{1-\frac{N}{d_{2} P}} \\
& \approx \rho 2^{-C} \sqrt{\frac{N}{d_{1} P}} \sqrt{\frac{N}{d_{2} P}}+\left[1-\frac{N}{2 d_{1} P} 2^{-2 C}\right]\left[1-\frac{N}{2 d_{2} P}\right] \\
& \approx \rho 2^{-C} \sqrt{\frac{N}{d_{1} P}} \sqrt{\frac{N}{d_{2} P}}+1-\frac{N}{2 P}\left(\frac{2^{-2 C}}{d_{1}}+\frac{1}{d_{2}}\right),
\end{aligned}
$$

where in both approximation steps we use that $\frac{N}{d_{\nu} P} \ll 1, \nu=1,2$.

Next, let $d_{2}=d$ and $d_{1}=\alpha d$ in which case the r.h.s. of (36) yields

$$
\varrho_{\mathrm{sep} 1}^{*}=1-\frac{N}{2 P d}\left(\frac{2^{-2 C}}{\alpha}+1\right)\left(1-\rho^{2}\right),
$$

while the r.h.s. of 37 yields

$$
\varrho_{\mathrm{VQ}}^{*} \geq \rho \frac{2^{-C}}{\sqrt{\alpha}} \frac{N}{d P}+1-\frac{N}{2 P d}\left(\frac{2^{-2 C}}{\alpha}+1\right) .
$$

The r.h.s. of (39) is strictly larger than the r.h.s. of (38) as long as

$$
\rho<2 \cdot \frac{2^{-C} \sqrt{\alpha}}{2^{-2 C}+\alpha} \text {. }
$$

It is easy to verify that $\alpha=2^{-2 C}$ satisfies (40).

We conclude that: 
Corollary 7 With fixed conferencing capacity and symmetric average-power constraints, for high-SNR, and $\left(d_{1}, d_{2}\right)$ such that $\lim _{N \rightarrow 0} \frac{N}{d_{1} P}=0$ and $\lim _{N \rightarrow 0} \frac{N}{d_{2} P}=0$, separation scheme 1 is suboptimal in the sense of attaining the optimal $d_{1} d_{2}$ given the system parameters $(\rho, P, N)$.

\section{Proof of THEOREM 1}

Lemma 1 For a multiple-access channel with unidirectional conferencing, let $\left\{X_{1, k}\right\},\left\{X_{2, k}\right\}$ and $\left\{Y_{k}\right\}$ be the channel inputs and channel outputs of a coding scheme achieving a distortion pair $\left(D_{1}, D_{2}\right)$. Then, for every $\delta>0$ there exists an $n_{0}(\delta)>0$ such that for all $n>n_{0}(\delta)$

$$
\begin{aligned}
n R_{S_{1}, S_{2}}\left(D_{1}+\delta, D_{2}+\delta\right) & \leq \sum_{k=1}^{n} I\left(X_{1, k}, X_{2, k} ; Y_{k}\right) \\
n R_{S_{2} \mid S_{1}}\left(D_{2}+\delta\right) & \leq \sum_{k=1}^{n} I\left(X_{2, k} ; Y_{k} \mid X_{1, k}, U_{k}\right),
\end{aligned}
$$

for $p_{X_{1, k} X_{2, k} U_{k}}=p_{U_{k}} p_{X_{1, k} \mid U_{k}} p_{X_{2, k} \mid U_{k}}$.

Proof: By the definition of an achievable distortion pair $\left(D_{1}, D_{2}\right)$ and the monotonicity of $R_{S_{1}, S_{2}}\left(\Delta_{1}, \Delta_{2}\right)$ in $\left(\Delta_{1}, \Delta_{2}\right)$, for any $\delta>0$ there exists an $n_{0}(\delta)>0$ such that for every $n>n_{0}(\delta)$

$$
n R_{S_{1}, S_{2}}\left(D_{1}+\delta, D_{2}+\delta\right) \leq I\left(\mathbf{S}_{1}, \mathbf{S}_{2} ; \mathbf{Y}\right),
$$

as reported in [2, Appendix I]. Next,

$$
\begin{aligned}
I\left(\mathbf{S}_{1}, \mathbf{S}_{2} ; \mathbf{Y}\right) & =h(\mathbf{Y})-h\left(\mathbf{Y} \mid \mathbf{S}_{1}, \mathbf{S}_{2}\right) \\
& =h(\mathbf{Y})-\sum_{k=1}^{n} h\left(Y_{k} \mid \mathbf{S}_{1}, \mathbf{S}_{2}, Y^{k-1}\right) \\
& \stackrel{(a)}{=} h(\mathbf{Y})-\sum_{k=1}^{n} h\left(Y_{k} \mid \mathbf{S}_{1}, \mathbf{S}_{2}, Y^{k-1}, W, X_{1, k}, X_{2, k}\right) \\
& \stackrel{(b)}{=} h(\mathbf{Y})-\sum_{k=1}^{n} h\left(Y_{k} \mid X_{1, k}, X_{2, k}\right) \\
& \leq \sum_{k=1}^{n} h\left(Y_{k}\right)-\sum_{k=1}^{n} h\left(Y_{k} \mid X_{1, k}, X_{2, k}\right) \\
& =\sum_{k=1}^{n} I\left(X_{1, k}, X_{2, k} ; Y_{k}\right) .
\end{aligned}
$$

Here, (a) follows since $W$ is a deterministic function of $\mathbf{S}_{1}$ and by the encoding relations (4), while $(b)$ follows since $Y_{k} \multimap\left(X_{1, k}, X_{2, k}\right) \multimap\left(\mathbf{S}_{1}, \mathbf{S}_{2}, Y^{k-1}, W\right)$ is a Markov chain. The combination of (43) and (44) establishes (41).

In a similar way

$$
n R_{S_{2} \mid S_{1}}\left(D_{2}+\delta\right) \leq I\left(\mathbf{S}_{2} ; \mathbf{Y} \mid \mathbf{S}_{1}\right),
$$

as reported in [2, Appendix I]. Next,

$$
\begin{aligned}
I\left(\mathbf{S}_{2} ; \mathbf{Y} \mid \mathbf{S}_{1}\right) & =h\left(\mathbf{Y} \mid \mathbf{S}_{1}\right)-h\left(\mathbf{Y} \mid \mathbf{S}_{1}, \mathbf{S}_{2}\right) \\
& \stackrel{(c)}{=} h\left(\mathbf{Y} \mid \mathbf{S}_{1}, W\right)-\sum_{k=1}^{n} h\left(Y_{k} \mid \mathbf{S}_{1}, \mathbf{S}_{2}, Y^{k-1}, W\right)
\end{aligned}
$$




$$
\begin{aligned}
& \stackrel{(d)}{=} \sum_{k=1}^{n} h\left(Y_{k} \mid \mathbf{S}_{1}, W, Y^{k-1}, X_{1, k}\right)-\sum_{k=1}^{n} h\left(Y_{k} \mid \mathbf{S}_{1}, \mathbf{S}_{2}, W, Y^{k-1}, X_{1, k}, X_{2, k}\right) \\
& \leq \sum_{k=1}^{n} h\left(Y_{k} \mid \mathbf{S}_{1}, W, X_{1, k}\right)-\sum_{k=1}^{n} h\left(Y_{k} \mid \mathbf{S}_{1}, \mathbf{S}_{2}, W, Y^{k-1}, X_{1, k}, X_{2, k}\right) \\
& \stackrel{(e)}{=} \sum_{k=1}^{n} h\left(Y_{k} \mid \mathbf{S}_{1}, W, X_{1, k}\right)-\sum_{k=1}^{n} h\left(Y_{k} \mid \mathbf{S}_{1}, W, X_{1, k}, X_{2, k}\right) \\
& =\sum_{k=1}^{n} I\left(X_{2, k} ; Y_{k} \mid X_{1, k}, \mathbf{S}_{1}, W\right) \\
& =\sum_{k=1}^{n} I\left(X_{2, k} ; Y_{k} \mid X_{1, k}, U_{k}\right) .
\end{aligned}
$$

Here, $(c)$ follows since $W$ is a deterministic function of $\mathbf{S}_{1} ;(d)$ follows by the encoding relations (47); and (e) follows since $Y_{k} \multimap-\left(X_{1, k}, X_{2, k}\right) \multimap\left(\mathbf{S}_{1}, \mathbf{S}_{2}, Y^{k-1}, W\right)$ is a Markov chain. Furthermore, in the last step we've defined $U_{k}=\left(\mathbf{S}_{1}, W\right)$ in which case, by the definition of the encoding relation $\mathbf{X}_{1}=\varphi_{1}^{(n)}\left(\mathbf{S}_{1}\right), U_{k}$ satisfies the Markov chain $X_{1, k} \multimap-U_{k} \multimap X_{2, k}$. The combination of (46) and (45) establishes (42).

Lemma 2 For a multiple-access channel with unidirectional conferencing, let the sequences $\left\{X_{1, k}\right\}$ and $\left\{X_{2, k}\right\}$ satisfy $\frac{1}{n} \sum_{k=1}^{n} \mathrm{E}\left[X_{\nu, k}^{2}\right] \leq P_{\nu}, \nu=1,2$. Let $Y_{k}=X_{1, k}+X_{2, k}+Z_{k}$, where $\left\{Z_{k}\right\}$ are IID zero-mean variance- $N$ Gaussian, and $Z_{k}$ is independent of $\left(X_{1, k}, X_{2, k}\right)$ for every $k$. Define $\varrho\left(\mathbf{X}_{1}, \mathbf{X}_{2}\right) \in[0,1]$ by

$$
\varrho\left(\mathbf{X}_{1}, \mathbf{X}_{2}\right)=\frac{\left|\frac{1}{n} \sum_{k=1}^{n} \mathrm{E}\left[X_{1, k} X_{2, k}\right]\right|}{\sqrt{\left(\frac{1}{n} \sum_{k=1}^{n} \mathrm{E}\left[X_{1, k}^{2}\right]\right)\left(\frac{1}{n} \sum_{k=1}^{n} \mathrm{E}\left[X_{2, k}^{2}\right]\right)}} .
$$

Then,

$$
\begin{aligned}
\sum_{k=1}^{n} I\left(X_{1, k}, X_{2, k} ; Y_{k}\right) & \leq \frac{n}{2} \log _{2}\left(1+\frac{P_{1}+P_{2}+2 \varrho\left(\mathbf{X}_{1}, \mathbf{X}_{2}\right) \sqrt{P_{1} P_{2}}}{N}\right) \\
\sum_{k=1}^{n} I\left(X_{2, k} ; Y_{k} \mid X_{1, k}, U_{k}\right) & \leq \frac{n}{2} \log _{2}\left(1+\frac{\operatorname{Var}\left(X_{2} \mid U\right)}{N}\right)
\end{aligned}
$$

for a Gaussian Markov triple $X_{1} \multimap U \multimap-X_{2}$.

Proof: By the Max-Entropy Theorem [15, Theorem 11.1.1] and the fact that the variance is always smaller than or equal to the second moment:

$$
\begin{aligned}
I\left(X_{1}, X_{2} ; Y\right) & \leq \frac{1}{2} \log _{2}\left(1+\frac{\operatorname{Var}\left(X_{1}+X_{2}\right)}{N}\right) \\
& \leq \frac{1}{2} \log _{2}\left(1+\frac{\mathrm{E}\left[\left(X_{1}+X_{2}\right)^{2}\right]}{N}\right) \\
& =\frac{1}{2} \log _{2}\left(1+\frac{\mathrm{E}\left[X_{1}^{2}\right]+\mathrm{E}\left[X_{2}^{2}\right]+2 \mathrm{E}\left[X_{1} X_{2}\right]}{N}\right) .
\end{aligned}
$$

This step reduces the multiple access problem to the problem of transmitting the source $\left(\mathbf{S}_{1}, \mathbf{S}_{2}\right)$ over a point to point AWGN channel of input power constraint $\mathrm{E}\left[\left(X_{1}+X_{2}\right)^{2}\right]$. The first inequality (48) follows now from the proof of the converse in [16, Section III] using Jensen's inequality. 
For the second inequality (49), again apply the Max-Entropy Theorem conditioned on $U=u$, and then use Jensen's inequality

$$
\begin{aligned}
I\left(X_{2} ; Y \mid X_{1}, U\right) & \leq \int \frac{1}{2} \log _{2}\left(1+\frac{\operatorname{Var}\left(X_{2} \mid U=u\right)}{N}\right) \mathrm{d} P_{U}(u) \\
& \leq \frac{1}{2} \log _{2}\left(1+\frac{\operatorname{Var}\left(X_{2} \mid U\right)}{N}\right) .
\end{aligned}
$$

It remains to show that for evaluating the upper bound it is sufficient to consider only Gaussian distributions. The proof follows the same lines as the proof of the main result in [10] and is omitted (see also [17, Lemma 3.15, and Appendix B.2]).

The last step in evaluating the upper bound, follows from two results from Maximum Correlation Theory, which are stated now. First, we recall Witsenhausen's lemma.

Lemma 3 [18 Theorem 1, p. 105] Consider a sequence of independent (across the time) pairs of random variables $\left\{\left(X_{k}, Y_{k}\right)\right\}$ and two Borel measurable arbitrary functions $g_{1, k}, g_{2, k}: \mathbb{R} \rightarrow \mathbb{R}$ satisfying

$$
\begin{aligned}
\mathrm{E}\left[g_{1, k}\left(X_{k}\right)\right] & =\mathrm{E}\left[g_{2, k}\left(Y_{k}\right)\right]=0, \\
\mathrm{E}\left[\left(g_{1, k}\left(X_{k}\right)\right)^{2}\right] & =\mathrm{E}\left[\left(g_{2, k}\left(Y_{k}\right)\right)^{2}\right]=1 .
\end{aligned}
$$

Define

$$
\varrho^{*} \triangleq \sup _{\substack{g_{1, k}, g_{2, k} \\ 1 \leq k \leq n}} \mathrm{E}\left[g_{1, k}\left(X_{k}\right) g_{2, k}\left(Y_{k}\right)\right]
$$

Then, for any two Borel measurable arbitrary functions $g_{1}^{(n)}, g_{2}^{(n)}: \mathbb{R}^{n} \rightarrow \mathbb{R}$ satisfying

$$
\begin{aligned}
\mathrm{E}\left[g_{1}^{(n)}(\mathbf{X})\right] & =\mathrm{E}\left[g_{2}^{(n)}(\mathbf{Y})\right]=0, \\
\mathrm{E}\left[\left(g_{1}^{(n)}(\mathbf{X})\right)^{2}\right] & =\mathrm{E}\left[\left(g_{2}^{(n)}(\mathbf{Y})\right)^{2}\right]=1,
\end{aligned}
$$

and for length-n sequences $\mathbf{X}$ and $\mathbf{Y}$, we have

$$
\sup _{g_{1}^{(n)}, g_{2}^{(n)}} \mathrm{E}\left[g_{1}^{(n)}(\mathbf{X}) g_{2}^{(n)}(\mathbf{Y})\right] \leq \varrho^{*}
$$

When $\left\{\left(X_{k}, Y_{k}\right)\right\}$ is IID, we define $\varrho^{*}=\varrho(X, Y)$ where

$$
\varrho(X, Y) \triangleq \sup _{g_{1}, g_{2}} \mathrm{E}\left[g_{1}(X) g_{2}(Y)\right] \text {. }
$$

The second result states that when $\left(X_{k}, Y_{k}\right)$ is a bivariate Gaussian, the supremum in (50) is obtained by linear mappings, as stated in the following lemma.

Lemma 4 [19. Lemma 10.2, p. 182] Consider two jointly Gaussian random variables $W_{1, k}$ and $W_{2, k}$ with correlation coefficient $\rho_{k}$. Then,

$$
\sup _{g_{1, k}, g_{2, k}} \mathrm{E}\left[g_{1, k}\left(W_{1, k}\right) g_{2, k}\left(W_{2, k}\right)\right]=\left|\rho_{k}\right|,
$$

where the supremum is taken over all functions $g_{i, k}: \mathbb{R} \rightarrow \mathbb{R}$, satisfying $\mathrm{E}\left[g_{i, k}\left(W_{i, k}\right)\right]=0$ and $\mathrm{E}\left[\left(g_{i, k}\left(W_{i, k}\right)\right)^{2}\right]=1, i \in\{1,2\}$. 
Using Witsenhausen's lemma we may upper-bound $\varrho\left(\mathbf{X}_{1}, \mathbf{X}_{2}\right)$ as follows

$$
\begin{aligned}
\varrho\left(\mathbf{X}_{1}, \mathbf{X}_{2}\right) & \leq \sup _{\substack{\varphi_{1}^{(n)}, \varphi_{2}^{(n)} \\
1 \leq k \leq n}} \mathrm{E}\left[\varphi_{1, k}^{(n)}\left(\mathbf{S}_{1}\right) \varphi_{2, k}^{(n)}\left(\mathbf{S}_{2}, W\right)\right] \\
& =\sup _{\substack{\varphi_{1}^{(n)}, \varphi_{2}^{(n)} \\
1 \leq k \leq n}} \mathrm{E}\left[\varphi_{1, k}^{(n)}\left(\mathbf{S}_{1}\right) \varphi_{2, k}^{(n)}\left(\mathbf{S}_{2}, f^{(n)}\left(\mathbf{S}_{1}\right)\right)\right] \\
& \stackrel{(a)}{\leq} \sup _{\varphi_{1}^{(n)}, \varphi_{2}^{(n)}} \mathrm{E}\left[\varphi_{1, k}^{(n)}\left(\mathbf{S}_{1}\right) \varphi_{2, k}^{(n)}\left(\mathbf{S}_{2}, \mathbf{S}_{1}\right)\right] \\
& \stackrel{(b)}{\leq} \sup _{\varphi_{1, k}, \varphi_{2, k}} \mathrm{E}\left[\varphi_{1, k}\left(S_{1, k}\right) \varphi_{2, k}\left(S_{2, k}, S_{1, k}\right)\right] .
\end{aligned}
$$

Here, $(a)$ follows since $f^{(n)}: \mathbb{R}^{n} \rightarrow \mathcal{W}$ is a deterministic function of $\mathbf{S}_{1}$, and (b) follows since $\left(\mathbf{S}_{1}, \mathbf{S}_{2}\right)$ is IID generated, hence Lemma 3 applies.

Next, define

$$
\sup _{\varphi_{1}, \varphi_{2}} \mathrm{E}\left[\varphi_{1}\left(S_{1}\right) \varphi_{2}\left(S_{2}, S_{1}\right)\right] \triangleq \varrho\left(S_{1},\left(S_{1}, S_{2}\right)\right) .
$$

Then

$$
\begin{aligned}
\varrho\left(S_{1},\left(S_{1}, S_{2}\right)\right) & =\varrho\left(S_{1},\left(S_{1}, S_{2}-\rho S_{1}\right)\right) \\
& \stackrel{(c)}{=} \varrho\left(S_{1}, S_{1}\right) \stackrel{(d)}{=} \varrho\left(S_{1}, S_{1}\right)+\varrho\left(S_{1}, S_{2}-\rho S_{1}\right) .
\end{aligned}
$$

Here,

(c) follows since conditioned on $S_{1}$, the random variable $S_{2}-\rho S_{1}$ is independent of $S_{1}$ and therefore

$$
\mathrm{E}\left[\varphi\left(S_{1}\right) \mid S_{1}, S_{2}-\rho S_{1}\right]=\mathrm{E}\left[\varphi\left(S_{1}\right) \mid S_{1}\right],
$$

in which case $(c)$ follows by the fact that if $X \multimap Y \multimap Z$ then $\varrho(X,(Y, Z))=\varrho(X, Y)$ (see [20, Proof of inequality (7)]).

(d) follows since $\varrho\left(S_{1}, S_{2}-\rho S_{1}\right)=0$ due to the fact that $S_{2}-\rho S_{1}$ is uncorrelated with $S_{1}$.

Consider the maximiziation of $\varrho\left(\mathbf{X}_{1}, \mathbf{X}_{2}\right)$ subject to the conditional rate-distortion constraint following from (42) and (49),

$$
R_{S_{2} \mid S_{1}}\left(D_{2}\right) \leq \frac{1}{2} \log _{2}\left(1+\frac{\operatorname{Var}\left(X_{2} \mid U\right)}{N}\right) .
$$

- Recall that the upper bound on the r.h.s. of (53) is attained by jointly Gaussian $\left(X_{1}, U, X_{2}\right)$.

- Conditioned on $S_{1}$ the energy-distortion tradeoff for attaining $R_{S_{2} \mid S_{1}}\left(D_{2}\right)$ is achieved by uncoded transmission of $S_{2}-\rho S_{1}$ by Encoder 2. Moreover, by (52) any linear function of $S_{2}-\rho S_{1}$ which Encoder 2 transmits does not interfere with the correlation that is built via the transmission of $S_{1}$ by both encoders.

- For a jointly Gaussian $\left(X_{1}, U, X_{2}\right)$ such that $X_{1} \multimap U \multimap X_{2}$ we have $\operatorname{Var}\left(X_{2} \mid U\right) \leq$ $\operatorname{Var}\left(X_{2} \mid X_{1}\right)$.

- We use a perturbation argument to argue that uncoded transmission of $S_{1}$ at both encoders maximizes $\varrho\left(\mathbf{X}_{1}, \mathbf{X}_{2}\right)$. This is true since by [20, Theorem 1] if $Z$ is independent of the pair $(X, Y)$ then $\varrho(X, Y+\lambda Z), \lambda \in \mathbb{R}$ is continuous at $\lambda=0$. Suppose that Encoder 2 acquires via the conference channel the sequence $\tilde{\mathbf{S}}_{1}$ where $\tilde{S}_{1, k}=S_{1, k}+\lambda \tilde{Z}_{k}, \lambda \in \mathbb{R}$ and $\left\{\tilde{Z}_{k}\right\}$ consists of IID zero mean variance $\tilde{N}$ Gaussians that are independent of the source sequence. By Lemmas 3 and 4 uncoded transmission of $\left\{S_{1, k}\right\}$ by Encoder 1 and 


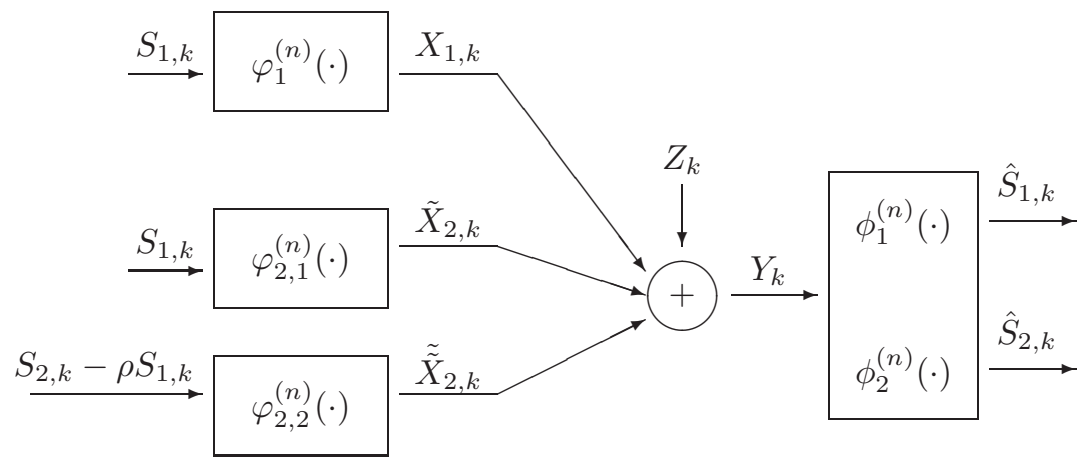

Fig. 8. Decomposition of Encoder 2 for evaluation of the maximum correlation with $C_{12}=\infty$

$\left\{\tilde{S}_{1, k}\right\}$ by Encoder 2 maximizes $\varrho\left(\mathbf{X}_{1}, \mathbf{X}_{2}\right)$. Since the rate-distortion region is a continuous function of $\lambda^{2} \tilde{N}$, the limit of the rate-distortion region attained by the above strategy as $\lambda \rightarrow 0$ converges to the solution to the constrained maximum of (51).

Consequently, for the solution to the constrained maximum of (51) we may assume that Encoder 2 is split into two separate sub-encoders, with respective inputs $\left(S_{1}, S_{2}-\rho S_{1}\right)$, respective outputs $\left(\tilde{X}_{2, k}, \tilde{X}_{2, k}\right)$ which are linear functions of the inputs and aggregate normalized power constraint

$$
\frac{1}{n} \mathrm{E}\left[\sum_{k=1}^{n}\left(\tilde{X}_{2, k}+\tilde{\tilde{X}}_{2, k}\right)^{2}\right] \leq 1 .
$$

This decomposition is shown in Fig. 8

Thus, for $0 \leq \beta \leq 1$, consider the linear mappings

$$
\begin{aligned}
\varphi_{1}\left(S_{1}\right) & =\frac{1}{\sigma} S_{1} \\
\varphi_{2}\left(S_{1}, S_{2}\right) & =\frac{\sqrt{\bar{\beta}}}{\sigma}\left(S_{2}-\rho S_{1}\right)+\frac{\sqrt{\beta\left(1-\rho^{2}\right)+\rho^{2}}}{\sigma} S_{1} \\
& =\frac{\sqrt{\bar{\beta}}}{\sigma} S_{2}+\frac{1}{\sigma}\left[\sqrt{\rho^{2} \bar{\beta}+\beta}-\sqrt{\rho^{2} \bar{\beta}}\right] S_{1} .
\end{aligned}
$$

It can be verified that, as required by Lemma 3 ,

$$
\begin{aligned}
\mathrm{E}\left[\varphi_{1}\left(S_{1}\right)\right] & =\mathrm{E}\left[\varphi_{2}\left(S_{1}, S_{2}\right)\right]=0 \\
\mathrm{E}\left[\left(\varphi_{1}\left(S_{1}\right)\right)^{2}\right] & =\mathrm{E}\left[\left(\varphi_{2}\left(S_{1}, S_{2}\right)\right)^{2}\right]=1,
\end{aligned}
$$

while

$$
\mathrm{E}\left[\varphi_{1}\left(S_{1}\right) \varphi_{2}\left(S_{2}, S_{1}\right)\right]=\sqrt{\rho^{2} \bar{\beta}+\beta} .
$$

Furthermore, for this set of linear mappings the random variable $U$ which satisfies

$\varphi_{1}\left(S_{1}\right) \multimap U \multimap \varphi_{2}\left(S_{1}, S_{2}\right)$, and is jointly Gaussian with $\left(\varphi_{1}\left(S_{1}\right), \varphi_{2}\left(S_{1}, S_{2}\right)\right)$, is $U=S_{1}$ in which case

$$
\operatorname{Var}\left(X_{2} \mid U\right)=\operatorname{Var}\left(\varphi_{2}\left(S_{1}, S_{2}\right) \mid U\right)=\frac{\bar{\beta}}{\sigma^{2}} \operatorname{Var}\left(S_{2} \mid S_{1}\right)=\bar{\beta}\left(1-\rho^{2}\right)
$$

Thus, the set of laws over which $\varrho\left(\mathbf{X}_{1}, \mathbf{X}_{2}\right)$ is maximized are those for which $U=X_{1}$ hence they simultaneously maximize the r.h.s. of (49) as well.

The combination of (41), (48), (51) and (54) establishes the upper bound (9) in Theorem 1. The combination of (42), (49), and (55) establishes the upper bound (10) in Theorem 1 


\section{Proof of Theorem 2}

\section{A. Coding scheme}

Fix some $\epsilon>0$ and a rate tuple $\left(R_{1}, R_{2}, R_{\mathrm{c}}\right)$.

Code Construction: Three codebooks $\mathcal{C}_{1}, \mathcal{C}_{2}$ and $\mathcal{C}_{c}$ are generated independently. Codebook $\mathcal{C}_{i}, i \in 1,2$, consists of $2^{n R_{i}}$ codewords $\left\{\mathbf{U}_{i}(1), \mathbf{U}_{i}(2), \ldots, \mathbf{U}_{i}\left(2^{n R_{i}}\right)\right\}$. The codewords are drawn independently uniformly over the surface of the centered $\mathbb{R}^{n}$-sphere $\mathcal{S}_{i}$ of radius $r_{i}=\sqrt{n \sigma^{2}\left(1-2^{-2 R_{i}}\right)}$. Codebook $\mathcal{C}_{c}$, consists of $2^{n R_{\mathrm{c}}}$ codewords

$\left\{\mathbf{V}(1), \mathbf{V}(2), \ldots, \mathbf{V}\left(2^{n R_{c}}\right)\right\}$. The codewords are drawn independently uniformly over the surface of the centered $\mathbb{R}^{n}$-sphere $\mathcal{S}_{c}$ of radius $r_{c}=\sqrt{n \sigma^{2} 2^{-2 R_{1}}\left(1-2^{-2 R_{c}}\right)}$.

Partition randomly the codebook $\mathcal{C}_{c}$ into $\left(1-\rho^{2} 2^{-2 R_{1}}\left(1-2^{-2 R_{\mathrm{c}}}\right)\right)^{\frac{n}{2}} 2^{n\left(R_{\mathrm{c}}+\delta(\epsilon)\right)}$ bins, each of size

$$
M_{b} \triangleq\left(1-\rho^{2} 2^{-2 R_{1}}\left(1-2^{-2 R_{\mathrm{c}}}\right)\right)^{-\frac{n}{2}} 2^{-n \delta(\epsilon)},
$$

and for any codeword $\mathbf{v}(k)$ let $b(k)$ denote the index of the bin containing $\mathbf{v}(k)$.

For every $\mathbf{w}, \mathbf{v} \in \mathbb{R}^{n}$ where neither $\mathbf{w}$ nor $\mathbf{v}$ are the zero-sequence, denote the angle between $\mathbf{w}$ and $\mathbf{v}$ by $\varangle(\mathbf{w}, \mathbf{v})$. i.e.,

$$
\cos \varangle(\mathbf{w}, \mathbf{v}) \triangleq \frac{\langle\mathbf{w}, \mathbf{v}\rangle}{\|\mathbf{w}\|\|\mathbf{v}\|} .
$$

Encoding: Given the source sequences $\left(\mathbf{s}_{1}, \mathbf{s}_{2}\right)$, let $\mathcal{F}\left(\mathbf{s}_{i}, \mathcal{C}_{i}\right)$ be the set defined by

$$
\mathcal{F}\left(\mathbf{s}_{i}, \mathcal{C}_{i}\right) \triangleq\left\{\mathbf{u}_{i} \in \mathcal{C}_{i}:\left|\cos \varangle\left(\mathbf{s}_{i}, \mathbf{u}_{i}\right)-\sqrt{1-2^{-2 R_{i}}}\right| \leq \sqrt{1-2^{-2 R_{i}}} \epsilon\right\} .
$$

Encoder 1 vector-quantizes $\mathbf{s}_{1}$ in two steps as follows:

1) If $\mathcal{F}\left(\mathbf{s}_{1}, \mathcal{C}_{1}\right) \neq \emptyset$ it forms the vector $\mathbf{u}_{1}^{*}$ by choosing it as the codeword $\mathbf{u}_{1}\left(j^{*}\right) \in \mathcal{F}\left(\mathbf{s}_{1}, \mathcal{C}_{1}\right)$ where $j^{*}$ minimizes $\left|\cos \varangle\left(\mathbf{s}_{1}, \mathbf{u}_{1}(j)\right)-\sqrt{1-2^{-2 R_{1}}}\right|$, while if $\mathcal{F}\left(\mathbf{s}_{1}, \mathcal{C}_{1}\right)=\emptyset$ then $\mathbf{u}_{1}^{*}$ is the all-zero sequence.

2) Let

$$
\mathbf{Z}_{Q_{1}} \triangleq \mathbf{S}_{1}-\mathbf{U}_{1}^{*}
$$

Let $\mathcal{F}\left(\mathbf{z}_{\mathrm{Q}_{1}}, \mathcal{C}_{c}\right)$ be the set defined by

$$
\mathcal{F}\left(\mathbf{z}_{\mathrm{Q}_{1}}, \mathcal{C}_{c}\right) \triangleq\left\{\mathbf{v} \in \mathcal{C}_{c}:\left|\cos \varangle\left(\mathbf{z}_{\mathrm{Q}_{1}}, \mathbf{v}\right)-\sqrt{1-2^{-2 R_{\mathrm{c}}}}\right| \leq \sqrt{1-2^{-2 R_{\mathrm{c}}} \epsilon}\right\} .
$$

If $\mathcal{F}\left(\mathbf{z}_{\mathrm{Q}_{1}}, \mathcal{C}_{c}\right) \neq \emptyset$ it forms the vector $\mathbf{v}^{*}$ by choosing it as the codeword $\mathbf{v}\left(k^{*}\right) \in \mathcal{F}\left(\mathbf{z}_{\mathrm{Q}_{1}}, \mathcal{C}_{c}\right)$ where $k^{*}$ minimizes $\left|\cos \varangle\left(\mathbf{z}_{\mathrm{Q}_{1}}, \mathbf{v}(k)\right)-\sqrt{1-2^{-2 R_{c}}}\right|$, while if $\mathcal{F}\left(\mathbf{z}_{\mathrm{Q}_{1}}, \mathcal{C}_{c}\right)=\emptyset$ then $\mathbf{v}^{*}$ is the all-zero sequence.

The channel input $\mathbf{X}_{1}$ is now given by (12).

Since the codebooks $\mathcal{C}_{1}$ and $\mathcal{C}_{c}$ are drawn over the centered $\mathbb{R}^{n}$-spheres of radii $r_{1}=$ $\sqrt{\sigma^{2}\left(1-2^{-2 R_{1}}\right)}$ and $r_{c}=\sqrt{\sigma^{2} 2^{-2 R_{1}}\left(1-2^{-2 R_{c}}\right)}$, respectively, and (as shown in Lemma 42 ahead) the codewords $\mathbf{U}_{1}^{*}$ and $\mathbf{V}^{*}$ are uncorrelated, the channel input $\mathbf{X}_{1}$ satisfies the averagepower constraint.

Encoder 1 informs Encoder 2 on $\mathbf{v}\left(k^{*}\right)$ by sending Encoder 2 the bin-index $b\left(k^{*}\right)$ over the unidirectional conference channel.

Encoder 2 vector-quantizes $\mathbf{s}_{2}$ as follows:

If $\mathcal{F}\left(\mathbf{s}_{2}, \mathcal{C}_{2}\right) \neq \emptyset$ it forms the vector $\mathbf{u}_{2}^{*}$ by choosing it as the codeword $\mathbf{u}_{2}\left(j^{*}\right) \in \mathcal{F}\left(\mathbf{s}_{2}, \mathcal{C}_{2}\right)$ where $j^{*}$ minimizes $\left|\cos \varangle\left(\mathbf{s}_{2}, \mathbf{u}_{2}(j)\right)-\sqrt{1-2^{-2 R_{2}}}\right|$, while if $\mathcal{F}\left(\mathbf{s}_{2}, \mathcal{C}_{2}\right)=\emptyset$ then $\mathbf{u}_{2}^{*}$ is the all-zero sequence. 
Encoder 2 acquires the codeword $\mathbf{v}\left(k^{*}\right)$ by choosing among the codewords within bin $b\left(k^{*}\right)$ the codeword $\mathbf{v}\left(k^{*}\right)$ such that

$$
\left|\rho_{\mathbf{v}, \mathbf{s}_{2}}-\cos \varangle\left(\mathbf{v}\left(k^{*}\right), \mathbf{s}_{2}\right)\right| \leq 5 \epsilon,
$$

where $\rho_{\mathbf{v}, \mathbf{s}_{2}} \triangleq \rho \sqrt{2^{-2 R_{1}}\left(1-2^{-2 R_{\mathrm{c}}}\right)}$.

The channel input $\mathbf{X}_{2}$ is now given by (13). Since the codebooks $\mathcal{C}_{2}$ and $\mathcal{C}_{c}$ are drawn over the centered $\mathbb{R}^{n}$-spheres of radii $r_{1}=\sqrt{\sigma^{2}\left(1-2^{-2 R_{2}}\right)}$ and $r_{c}=\sqrt{\sigma^{2} 2^{-2 R_{1}}\left(1-2^{-2 R_{\mathrm{c}}}\right)}$, respectively, and (as shown in Lemma 43 ahead) the codewords $\mathbf{U}_{2}^{*}$ and $\mathbf{V}^{*}$ are correlated, the channel input $\mathbf{X}_{2}$ satisfies the average-power constraint.

Reconstruction: The receiver's estimate $\left(\hat{\mathbf{S}}_{1}, \hat{\mathbf{S}}_{2}\right)$ of the source pair $\left(\mathbf{S}_{1}, \mathbf{S}_{2}\right)$ is obtained via the channel output $\mathbf{Y}$ in two steps. First, the receiver makes a guess $\left(\hat{\mathbf{U}}_{1}, \hat{\mathbf{V}}, \hat{\mathbf{U}}_{2}\right)$ of the tuple $\left(\mathbf{U}_{1}^{*}, \mathbf{V}^{*}, \mathbf{U}_{2}^{*}\right)$ by choosing among all "jointly typical" tuples $\left(\mathbf{u}_{1}, \mathbf{v}, \mathbf{u}_{2}\right) \in \mathcal{C}_{1} \times \mathcal{C}_{c} \times \mathcal{C}_{2}$ the tuple whose linear combination $a_{1,1} \mathbf{U}_{1}+a_{2,1} \mathbf{U}_{2}+\left(a_{1,2}+a_{2,2}\right) \mathbf{V}$ has the smallest distance to the received sequence $\mathbf{Y}$. More formally, let $\overline{\mathcal{F}}\left(\mathcal{C}_{1}, \mathcal{C}_{c}, \mathcal{C}_{2}\right)$ be the set of triplets $\left(\mathbf{u}_{1}, \mathbf{v}, \mathbf{u}_{2}\right) \in$ $\mathcal{C}_{1} \times \mathcal{C}_{c} \times \mathcal{C}_{2}$ such that

$$
\begin{aligned}
\left|\tilde{\rho}-\cos \varangle\left(\mathbf{u}_{1}, \mathbf{u}_{2}\right)\right| & \leq 7 \epsilon \\
\left|\bar{\rho}-\cos \varangle\left(\mathbf{v}, \mathbf{u}_{2}\right)\right| & \leq 7 \epsilon \\
\left|\cos \varangle\left(\mathbf{v}, \mathbf{u}_{1}\right)\right| & \leq 3 \epsilon,
\end{aligned}
$$

where $(\tilde{\rho}, \bar{\rho})$ are defined in (19), and for any tuple $\left(\mathbf{u}_{1}, \mathbf{v}, \mathbf{u}_{2}\right)$ define

$$
\begin{aligned}
\mathbf{X}_{\mathbf{u}_{1}, \mathbf{v}, \mathbf{u}_{2}} & \triangleq a_{1,1} \mathbf{u}_{1}+a_{2,1} \mathbf{u}_{2}+\left(a_{1,2}+a_{2,2}\right) \mathbf{v} \\
& =a_{1,1} \mathbf{u}_{1}+a_{2,1} \mathbf{u}_{2}+\alpha \mathbf{v},
\end{aligned}
$$

where $\alpha \triangleq a_{1,2}+a_{2,2}$. Then the receiver forms its estimate by choosing

$$
\left(\hat{\mathbf{U}}_{1}, \hat{\mathbf{V}}, \hat{\mathbf{U}}_{2}\right)=\arg \min _{\left(\mathbf{u}_{1}, \mathbf{v}, \mathbf{u}_{2}\right) \in \overline{\mathcal{F}}\left(\mathcal{C}_{1}, \mathcal{C}_{c}, \mathcal{C}_{2}\right)}\left\|\mathbf{Y}-\mathbf{X}_{\mathbf{u}_{1}, \mathbf{v}, \mathbf{u}_{2}}\right\|^{2} .
$$

If the channel output $\mathbf{Y}$ and the codebooks are such that there doesn't exist a member in $\overline{\mathcal{F}}\left(\mathcal{C}_{1}, \mathcal{C}_{c}, \mathcal{C}_{2}\right)$ that minimizes the r.h.s. in $[61)$, then $\left(\hat{\mathbf{U}}_{1}, \hat{\mathbf{V}}, \hat{\mathbf{U}}_{2}\right)$ are chosen to be the all-zero sequences.

In the second step, the receiver forms its estimates $\left(\hat{\mathbf{S}}_{1}, \hat{\mathbf{S}}_{2}\right)$ of the source pair $\left(\mathbf{S}_{1}, \mathbf{S}_{2}\right)$ according to (14).

\section{B. Expected distortion}

Similarly to [1], to analyze the expected distortion we first show that, when the rate constraints (18) are satisfied, the asymptotic normalized distortion of the proposed scheme remains the same as that of a genie-aided scheme in which the genie provides the decoder with the triplet $\left(\mathbf{U}_{1}^{*}, \mathbf{V}^{*}, \mathbf{U}_{2}^{*}\right)$. The genie-aided decoder forms its estimate $\left(\hat{\mathbf{S}}_{1}^{\mathrm{G}}, \hat{\mathbf{S}}_{2}^{\mathrm{G}}\right)$ based on $\left(\mathbf{U}_{1}^{*}, \mathbf{V}^{*}, \mathbf{U}_{2}^{*}\right)$ according to (14) and ignores its guess $\left(\hat{\mathbf{U}}_{1}, \hat{\mathbf{V}}, \hat{\mathbf{U}}_{2}\right)$ produced in the first decoding step. Hence, $\left(\hat{\mathbf{S}}_{1}^{\mathrm{G}}, \hat{\mathbf{S}}_{2}^{\mathrm{G}}\right)$ is defined by

$$
\begin{aligned}
& \hat{\mathbf{S}}_{1}^{\mathrm{G}}=\gamma_{1,1} \mathbf{U}_{1}^{*}+\gamma_{1,2} \mathbf{U}_{2}^{*}+\gamma_{1,3} \mathbf{V}^{*} \\
& \hat{\mathbf{S}}_{2}^{\mathrm{G}}=\gamma_{2,1} \mathbf{U}_{1}^{*}+\gamma_{2,2} \mathbf{U}_{2}^{*}+\gamma_{2,3} \mathbf{V}^{*},
\end{aligned}
$$

with $\gamma_{1,1}, \gamma_{1,2}, \gamma_{1,3}, \gamma_{2,1}, \gamma_{2,2}, \gamma_{2,3}$ as in (15). 
Proposition 4 If $\left(R_{1}, R_{2}, R_{\mathrm{c}}\right)$ satisfy

$$
\begin{aligned}
R_{1} & <\frac{1}{2} \log \left(\frac{\bar{\beta}_{1} P_{1}\left(1-\tilde{\rho}^{2}\right)+N-\bar{\rho}^{2}\left(\bar{\beta}_{1} P_{1}+N\right)}{N\left(1-\tilde{\rho}^{2}\right)-\bar{\rho}^{2} N}\right) \\
R_{2} & <\frac{1}{2} \log \left(\frac{\bar{\beta}_{2} P_{2}\left(1-\tilde{\rho}^{2}-\bar{\rho}^{2}\right)+N}{N\left(1-\tilde{\rho}^{2}-\bar{\rho}^{2}\right)+\lambda_{2}}\right) \\
R_{\mathrm{c}} & <\frac{1}{2} \log \left(\frac{\eta^{2}\left(1-\tilde{\rho}^{2}-\bar{\rho}^{2}\right)+N\left(1-\tilde{\rho}^{2}\right)}{N\left(1-\tilde{\rho}^{2}-\bar{\rho}^{2}\right)+\lambda_{c}}\right) \\
R_{1}+R_{2} & <\frac{1}{2} \log \left(\frac{\lambda_{12}-\bar{\beta}_{2} P_{2} \bar{\rho}^{2}+N}{\left(1-\bar{\beta}_{2} P_{2} \bar{\rho}^{2} \lambda_{12}{ }^{-1}\right) N\left(1-\tilde{\rho}^{2}\right)}\right) \\
R_{1}+R_{\mathrm{c}} & <\frac{1}{2} \log \left(\frac{\left(\lambda_{1 c}+N\right)\left(\bar{\beta}_{1} P_{1}+\eta^{2}\right)}{\lambda_{1 c} N}\right) \\
R_{2}+R_{\mathrm{c}} & <\frac{1}{2} \log \left(\frac{\lambda_{2 c}-\bar{\beta}_{2} P_{2} \tilde{\rho}^{2}+N}{\left(1-\bar{\beta}_{2} P_{2} \tilde{\rho}^{2} \lambda_{2 c}{ }^{-1}\right) N\left(1-\bar{\rho}^{2}\right)}\right) \\
R_{1}+R_{2}+R_{\mathrm{c}}< & \frac{1}{2} \log \left(\frac{\lambda_{12}+2 \eta \bar{\rho} \sqrt{\bar{\beta}_{2} P_{2}}+\eta^{2}+N}{N\left(1-\tilde{\rho}^{2}\right)\left(1-\bar{\rho}^{2}\right)}\right) \\
C_{12} & >R_{\mathrm{c}}+\frac{1}{2} \log \left(1-\rho^{2} 2^{-2 R_{1}}\left(1-2^{-2 R_{\mathrm{c}}}\right)\right),
\end{aligned}
$$

then

$$
\varlimsup_{n \rightarrow \infty} \frac{1}{n} \mathrm{E}\left[\left\|\mathbf{S}_{\nu}-\hat{\mathbf{S}}_{\nu}\right\|^{2}\right] \leq \varlimsup_{n \rightarrow \infty} \frac{1}{n} \mathrm{E}\left[\left\|\mathbf{S}_{\nu}-\hat{\mathbf{S}}_{\nu}^{\mathrm{G}}\right\|^{2}\right], \nu=1,2 .
$$

Proof: Follows from Proposition 6 (which appears in the Appendix) by first letting $n \rightarrow \infty$ and then $\epsilon \rightarrow 0$ and $\delta \rightarrow 0$.

By Proposition 4 to analyze the distortion achievable by our scheme it suffices to analyze the genie-aided scheme.

Proposition 5 The distortion pair $\left(D_{1}, D_{2}\right)$ of the genie-aided scheme satisfies

$$
\begin{aligned}
& D_{1} \leq \sigma^{2} 2^{-2\left(R_{1}+R_{\mathrm{c}}\right)} \frac{1-\rho^{2}\left(1-2^{-2 R_{2}}\right)}{1-\rho^{2}\left(1-2^{-2 R_{2}}\right)\left(1-2^{-2\left(R_{1}+R_{\mathrm{c}}\right)}\right)}+\xi^{\prime}(\delta, \epsilon) \\
& D_{2} \leq \sigma^{2} 2^{-2 R_{2}} \frac{1-\rho^{2}\left(1-2^{-2\left(R_{1}+R_{\mathrm{c}}\right)}\right)}{1-\rho^{2}\left(1-2^{-2 R_{2}}\right)\left(1-2^{-2\left(R_{1}+R_{\mathrm{c}}\right)}\right)}+\xi^{\prime}(\delta, \epsilon),
\end{aligned}
$$

where $\lim _{\delta, \epsilon \rightarrow 0} \xi^{\prime}(\delta, \epsilon)=0$.

Proof: See Appendix.

\section{Proof of Proposition 3}

Let $\left(S_{1}, S_{2}\right)$ be a pair of zero-mean jointly Gaussian random variables with covariance matrix as per (2). Let $\mathcal{P}\left(D_{1}, D_{2}\right)$ be the set of triples $(U, V, W)$ jointly Gaussian with $\left(S_{1}, S_{2}\right)$ such that

1) $U \multimap\left(S_{2}, W\right) \multimap\left(S_{1}, W\right) \multimap V$ and $W \multimap S_{1} \multimap-S_{2}$ are Markov chains,

2) $\sigma_{S_{1} \mid U, V, W}^{2} \leq D_{1}, \sigma_{S_{2} \mid U, V, W}^{2} \leq D_{2}$. 
This set can be defined as follows. Independently of $\left(S_{1}, S_{2}\right)$ draw a triplet of independent random variables $N^{(w)} \sim N\left(0, \sigma_{N^{(w)}}^{2}\right), N^{(u)} \sim N\left(0, \sigma_{N^{(u)}}^{2}\right)$ and $N^{(v)} \sim N\left(0, \sigma_{N^{(v)}}^{2}\right)$, and define

$$
\begin{aligned}
W & =S_{1}+N^{(w)} \\
U & =a_{1 u} W+a_{2 u} S_{2}+N^{(u)} \\
V & =a_{1 v} W+a_{2 v} S_{1}+N^{(v)} .
\end{aligned}
$$

Then,

$$
\begin{aligned}
\mathcal{R}_{\mathrm{G}}\left(D_{1}, D_{2}\right)=\bigcup_{(U, V, W) \in \mathcal{P}\left(D_{1}, D_{2}\right)}\left\{\left(R_{0}, R_{1}, R_{2}\right):\right. & R_{0} \geq \frac{1}{2} \log \left(\frac{\sigma_{W \mid S_{2}}^{2}}{\sigma_{W \mid S_{1}}^{2}}\right) \\
R_{1} & \geq \frac{1}{2} \log \left(\frac{\sigma_{V \mid U, W}^{2}}{\sigma_{V \mid S_{1}, W}^{2}}\right) \\
R_{2} & \geq \frac{1}{2} \log \left(\frac{\sigma_{U \mid V, W}^{2}}{\sigma_{U \mid S_{2}, W}^{2}}\right) \\
R_{1}+R_{2} & \left.\geq \frac{1}{2} \log \left(\frac{\left|\mathrm{K}_{S S}\right|}{\left|\mathrm{K}_{S S \mid U V W}\right|}\right)\right\},
\end{aligned}
$$

where $\mathrm{K}_{S S \mid U V W}$ is the covariance matrix of $\left(S_{1}, S_{2}\right)$ conditioned on $(U, V, W)$.

Defining $\sigma_{u}^{2} \triangleq \frac{\sigma_{N(u)}^{2}}{a_{2 u}^{2}}, \sigma_{v}^{2} \triangleq \frac{\sigma_{N(v)}^{2}}{a_{2 v}^{2}}$ and $\sigma_{w}^{2} \triangleq \sigma_{N(w)}^{2}$ the result follows since

$$
\begin{aligned}
\frac{\sigma_{W \mid S_{2}}^{2}}{\sigma_{W \mid S_{1}}^{2}} & =1+\frac{\sigma^{2}\left(1-\rho^{2}\right)}{\sigma_{w}^{2}} \\
\frac{\sigma_{V \mid U, W}^{2}}{\sigma_{V \mid S_{1}, W}^{2}} & =1+\frac{\sigma^{2}}{\sigma_{v}^{2}} \frac{\left(1-\rho^{2}\right)+\frac{\sigma_{u}^{2}}{\sigma^{2}}}{1+\frac{\sigma^{2}\left(1-\rho^{2}\right)}{\sigma_{w}^{2}}+\sigma_{u}^{2}\left(\frac{1}{\sigma_{w}^{2}}+\frac{1}{\sigma^{2}}\right)} \\
\frac{\sigma_{U \mid V, W}^{2}}{\sigma_{U \mid S_{2}, W}^{2}} & =1+\frac{\sigma^{2}}{\sigma_{u}^{2}} \frac{1+\sigma^{2}\left(\frac{1}{\sigma_{w}^{2}}+\frac{1}{\sigma_{v}^{2}}\right)\left(1-\rho^{2}\right)}{1+\sigma^{2}\left(\frac{1}{\sigma_{w}^{2}}+\frac{1}{\sigma_{v}^{2}}\right)},
\end{aligned}
$$

and

$$
\begin{aligned}
\sigma_{S_{1} \mid U, V, W}^{2} & =\sigma^{2} \frac{1+\frac{\sigma^{2}}{\sigma_{u}^{2}}\left(1-\rho^{2}\right)}{\Delta} \\
\sigma_{S_{2} \mid U, V, W}^{2} & =\sigma^{2} \frac{1+\sigma^{2}\left(1-\rho^{2}\right)\left(\frac{1}{\sigma_{v}^{2}}+\frac{1}{\sigma_{w}^{2}}\right)}{\Delta}
\end{aligned}
$$

where

$$
\begin{aligned}
\Delta & \triangleq 1+\frac{\sigma^{2}}{\sigma_{u}^{2}}+\sigma^{2}\left(1+\frac{\sigma^{2}}{\sigma_{u}^{2}}\left(1-\rho^{2}\right)\right)\left(\frac{1}{\sigma_{v}^{2}}+\frac{1}{\sigma_{w}^{2}}\right) \\
& =\frac{\left|\mathrm{K}_{S S}\right|}{\left|\mathrm{K}_{S S \mid U V W}\right|} .
\end{aligned}
$$

VI. Proof of Theorem 3

The high-SNR asymptotics for the multiple-access problem, when $C_{12}=\infty$, can be obtained from the necessary condition for the achievability of a distortion pair $\left(D_{1}, D_{2}\right)$ in Theorem 1 , 
and from the sufficient conditions for the achievability of a distortion pair $\left(D_{1}, D_{2}\right)$ derived from Corollary 1.

First we recall the rate-distortion function of a bivariate Gaussian.

Theorem 4 [7],[1] Theorem III.1] The rate-distortion function $R_{S_{1}, S_{2}}\left(D_{1}, D_{2}\right)$ is given by

$$
R_{S_{1}, S_{2}}\left(D_{1}, D_{2}\right)= \begin{cases}\frac{1}{2} \log _{2}^{+}\left(\frac{\sigma^{2}}{D_{\min }}\right) & \text { if }\left(D_{1}, D_{2}\right) \in \mathscr{D}_{1} \\ \frac{1}{2} \log _{2}^{+}\left(\frac{\sigma^{4}\left(1-\rho^{2}\right)}{D_{1} D_{2}}\right) & \text { if }\left(D_{1}, D_{2}\right) \in \mathscr{D}_{2} \\ \frac{1}{2} \log _{2}^{+}\left(\frac{\sigma^{4}\left(1-\rho^{2}\right)}{D_{1} D_{2}-\left(\rho \sigma^{2}-\varrho\left(D_{1}, D_{2}\right)\right)^{2}}\right) & \text { if }\left(D_{1}, D_{2}\right) \in \mathscr{D}_{3}\end{cases}
$$

where

$$
\varrho\left(D_{1}, D_{2}\right) \triangleq \sqrt{\left(\sigma^{2}-D_{1}\right)\left(\sigma^{2}-D_{2}\right)},
$$

$\log _{2}^{+}(x) \triangleq \max \left\{0, \log _{2}(x)\right\}$, and $D_{\text {min }} \triangleq \min \left\{D_{1}, D_{2}\right\}$, and the regions $\mathscr{D}_{i}, i=1,2,3$ are defined by

$$
\begin{aligned}
\mathscr{D}_{1} \triangleq & \left(D_{1}, D_{2}\right):\left(0 \leq D_{1} \leq v, D_{2} \geq v+\rho^{2} D_{1}\right) \text { or } \\
& \left.\left(v<D_{1} \leq \sigma^{2}, v+\rho^{2} D_{1} \leq D_{2} \leq \frac{D_{1}-v}{\rho^{2}}\right)\right\} \\
\mathscr{D}_{2} \triangleq & \left\{\left(D_{1}, D_{2}\right): 0 \leq D_{1} \leq v, 0 \leq D_{2}<\left(v-D_{1}\right) \frac{\sigma^{2}}{\sigma^{2}-D_{1}}\right\} \\
\mathscr{D}_{3} \triangleq & \left\{\left(D_{1}, D_{2}\right):\left(0 \leq D_{1} \leq v,\left(v-D_{1}\right) \frac{\sigma^{2}}{\sigma^{2}-D_{1}} \leq D_{2}<v+\rho^{2} D_{1}\right)\right. \text { or } \\
& \left.\left(v<D_{1} \leq \sigma^{2}, \frac{D_{1}-v}{\rho^{2}}<D_{2}<v+\rho^{2} D_{1}\right)\right\},
\end{aligned}
$$

with $v \triangleq \sigma^{2}\left(1-\rho^{2}\right)$.

By Corollary 1 when the conferencing capacity is unlimited, it follows that any normalized distortion pair $\left(d_{1}, d_{2}\right)$ satisfying $d_{1} \leq 1, d_{2} \leq 1$ and

$$
\begin{aligned}
d_{1} & \geq \frac{N}{\delta_{1}^{2}} \\
d_{2} & \geq \frac{N}{\bar{\beta} P_{2}} \\
d_{1} d_{2} & =\frac{N\left(1-\breve{\rho}^{2}\right)}{P_{1}+P_{2}+2 \sqrt{\left(\breve{\rho}^{2} \bar{\beta}+\beta\right) P_{1} P_{2}}}, \quad 0 \leq \beta<1
\end{aligned}
$$

where $\breve{\rho}=\rho \sqrt{\left(1-d_{1}\right)\left(1-d_{2}\right)}$, is achievable.

Next, if

$$
\lim _{N \rightarrow 0} \frac{N}{d_{1} P_{1}}=0 \quad \text { and } \quad \lim _{N \rightarrow 0} \frac{N}{d_{2} P_{2}}=0,
$$

then (64) and (65) are satisfied for sufficiently small $N$ and some $0 \leq \beta<1$. Thus, for $N$ sufficiently small, any pair $\left(d_{1}, d_{2}\right)$ satisfying (66) and (67) is achievable provided that $\beta$ satisfies the constraint imposed by (65). However, if $\left(d_{1}, d_{2}\right)$ satisfies (66) then the following 
pair of inequalities holds

$$
d_{2} \leq \frac{N}{d_{1} P_{1}}, \quad \text { and } \quad d_{1} \leq \frac{N}{d_{2} P_{2}} .
$$

Combining (68) with the expression of $\breve{\rho}$ yields that if in addition to 66 the pair $\left(d_{1}, d_{2}\right)$ satisfies [67), then $\breve{\rho} \rightarrow \rho$ as $N \rightarrow 0$. In conclusion, the sufficient condition yields that, if a pair $\left(d_{1}, d_{2}\right)$ satisfies (66) and (67), then

$$
\lim _{N \rightarrow 0} \frac{P_{1}+P_{2}+2 \sqrt{\left(\rho^{2} \bar{\beta}+\beta\right)} \sqrt{P_{1} P_{2}}}{N} d_{1} d_{2} \leq\left(1-\rho^{2}\right) .
$$

Now, let $\left(D_{1}^{*}, D_{2}^{*}\right)$ be a distortion pair of an optimal scheme. Then, by the upper bound (9) in Theorem 1 we have that for some $0 \leq \beta<1$

$$
R_{S_{1}, S_{2}}\left(D_{1}^{*}, D_{2}^{*}\right) \leq \frac{1}{2} \log _{2}\left(1+\frac{P_{1}+P_{2}+2 \sqrt{\left(\rho^{2} \bar{\beta}+\beta\right)} \sqrt{P_{1} P_{2}}}{N}\right) .
$$

If $\left(D_{1}^{*}, D_{2}^{*}\right)$ satisfies

$$
\lim _{N \rightarrow 0} \frac{N}{D_{1}^{*} P_{1}}=0, \quad \text { and } \quad \lim _{N \rightarrow 0} \frac{N}{D_{2}^{*} P_{2}}=0,
$$

then for $N$ small enough

$$
R_{S_{1}, S_{2}}\left(D_{1}^{*}, D_{2}^{*}\right)=\frac{1}{2} \log _{2}^{+}\left(\frac{\sigma^{4}\left(1-\rho^{2}\right)}{D_{1}^{*} D_{2}^{*}}\right),
$$

by Theorem 4 and the fact that $\left(D_{1}^{*}, D_{2}^{*}\right) \in \mathscr{D}_{2}$. The combination of (70) and (72) implies that if $\left(D_{1}^{*}, D_{2}^{*}\right)$ satisfies $(71)$, then

$$
\lim _{N \rightarrow 0} \frac{P_{1}+P_{2}+2 \sqrt{\left(\rho^{2} \bar{\beta}+\beta\right)} \sqrt{P_{1} P_{2}}}{N} d_{1} d_{2} \geq\left(1-\rho^{2}\right) .
$$

Remark 5 To check consistency, note that for every $\left(D_{1}^{*}, D_{2}^{*}\right) \in \mathscr{D}_{2}$ the rate-distortion function $R_{S_{1}, S_{2}}\left(D_{1}^{*}, D_{2}^{*}\right)$ satisfies

$$
\begin{aligned}
R_{S_{1}, S_{2}}\left(D_{1}^{*}, D_{2}^{*}\right) & =\frac{1}{2} \log _{2}^{+}\left(\frac{\sigma^{4}\left(1-\rho^{2}\right)}{D_{1}^{*} D_{2}^{*}}\right) \\
& =\frac{1}{2} \log _{2}^{+}\left(\frac{\sigma^{2}}{D_{1}^{*}}\right)+\frac{1}{2} \log _{2}^{+}\left(\frac{\sigma^{2}\left(1-\rho^{2}\right)}{D_{2}^{*}}\right) \\
& =R_{S_{1}}\left(D_{1}^{*}\right)+R_{S_{2} \mid S_{1}}\left(D_{2}^{*}\right) .
\end{aligned}
$$

Consequently, as $N \rightarrow 0$, by the upper bound (10) in Theorem 1

$$
\frac{1}{2} \log _{2}^{+}\left(\frac{\sigma^{2}\left(1-\rho^{2}\right)}{D_{2}^{*}}\right) \leq \frac{1}{2} \log _{2}\left(\frac{\bar{\beta} P_{2}\left(1-\rho^{2}\right)}{N}\right)
$$

i.e. $D_{2}^{*} \geq \sigma^{2} \frac{N}{\bar{\beta} P_{2}}$ as assumed in (65).

The combination of (69) with (73) and (74) implies that the high-SNR asymptotics for the Gaussian MAC with unlimited unidirectional conferencing capacity satisfies, for some $0 \leq \beta \leq$ $1-\frac{N}{d_{2}^{*} P_{2}}$,

$$
\lim _{N \rightarrow 0} \frac{P_{1}+P_{2}+2 \sqrt{\left(\rho^{2} \bar{\beta}+\beta\right)} \sqrt{P_{1} P_{2}}}{N} d_{1}^{*} d_{2}^{*}=\left(1-\rho^{2}\right),
$$


provided that $d_{1}^{*} \leq 1, d_{2}^{*} \leq 1$, and that $\left(d_{1}^{*}, d_{2}^{*}\right)$ satisfy (24). It remains to optimize the correlation $\varrho(\beta)$ over $\beta$ subject to the constraint [65],

$$
\varrho^{*}=\sup _{\bar{\beta} \geq \frac{N}{d_{2}^{*} P_{2}}} \sqrt{\rho^{2} \bar{\beta}+\beta}=\sqrt{\rho^{2} \frac{N}{d_{2}^{*} P_{2}}+\left(1-\frac{N}{d_{2}^{*} P_{2}}\right)}=\sqrt{1-\frac{N\left(1-\rho^{2}\right)}{d_{2}^{*} P_{2}}},
$$

and clearly $\varrho_{\mathrm{VQ}}^{*}=\varrho^{*}-$ i.e. the maximal correlation attained by the VQ scheme equals $\varrho^{*}$ since it is the same function of $\beta$ and it is defined over the same domain.

This concludes the proof of Theorem 3

\section{Proof of Corollary 2}

By Proposition 1 and (22), when the conferencing capacity is unlimited, it follows that any normalized distortion pair $\left(d_{1}, d_{2}\right)$ satisfying $d_{1} \leq 1, d_{2} \leq 1$ and

$$
\begin{aligned}
d_{1} & \geq \frac{N\left(1-\rho^{2}\right)}{P_{1}+P_{2}+2 \sqrt{\beta P_{1} P_{2}}} \\
d_{2} & \geq \frac{N\left(1-\rho^{2}\right)}{\bar{\beta} P_{2}} \\
d_{1} d_{2} & =\frac{N\left(1-\rho^{2}\right) \gamma\left(d_{1}, d_{2}\right)}{2\left(P_{1}+P_{2}+2 \sqrt{\beta P_{1} P_{2}}\right)} \quad 0 \leq \beta<1
\end{aligned}
$$

where $\gamma\left(d_{1}, d_{2}\right)=1+\sqrt{1+\frac{4 \rho^{2} d_{1} d_{2}}{\left(1-\rho^{2}\right)^{2}}}$ is achievable.

Next, if

$$
\lim _{N \rightarrow 0} \frac{N}{d_{1} P_{1}}=0, \quad \text { and } \quad \lim _{N \rightarrow 0} \frac{N}{d_{2} P_{2}}=0,
$$

then (75) and (76) are satisfied for sufficiently small $N$ and some $0 \leq \beta<1$. Thus, for $N$ sufficiently small, any pair satisfying (77) and (78) is achievable provided that $\beta$ satisfies the constraint imposed by (76).

Since $\gamma\left(d_{1}, d_{2}\right) \geq 2$, a distortion pair $\left(d_{1}, d_{2}\right)$ is achievable by source-channel separation scheme 1 if, and only if,

$$
\lim _{N \rightarrow 0} \frac{P_{1}+P_{2}+2 \sqrt{\beta P_{1} P_{2}}}{N} d_{1} d_{2} \geq\left(1-\rho^{2}\right) .
$$

It remains to optimize the correlation $\varrho_{\operatorname{sep} 1}(\beta)$ over $\beta$ subject to the constraint $(76)$,

$$
\varrho_{\mathrm{sep} 1}^{*}=\sup _{\bar{\beta} \geq \frac{N\left(1-\rho^{2}\right)}{d_{2} P_{2}}} \sqrt{\beta}=\sqrt{1-\frac{N\left(1-\rho^{2}\right)}{d_{2} P_{2}}} .
$$

This concludes the proof of Corollary 2

\section{Proof OF COROLlary 5}

By Proposition 11 and [10], for a fixed conferencing capacity $C_{12}=C$, it follows that any normalized distortion pair $\left(d_{1}, d_{2}\right)$ satisfying $d_{1} \leq 1, d_{2} \leq 1$ and

$$
\begin{aligned}
d_{1} & \geq \frac{N}{\bar{\beta}_{1} P_{1}}\left(1-\rho^{2}\right) 2^{-2 C} \\
d_{2} & \geq \frac{N}{\bar{\beta}_{2} P_{2}}\left(1-\rho^{2}\right) \\
d_{1} d_{2} & =\frac{N\left(1-\rho^{2}\right)}{P_{1}+P_{2}+2 \sqrt{P_{1} P_{2}} \sqrt{\beta_{1} \beta_{2}}}, \quad 0 \leq \beta_{1}, \beta_{2}<1
\end{aligned}
$$


is achievable.

Next, if (27) holds then (79) and (80) are satisfied for sufficiently small $N$ and some $0 \leq$ $\beta_{1}, \beta_{2}<1$. Thus, for $N$ sufficiently small, any pair satisfying (27) and (81), is achievable provided that $\left(\beta_{1}, \beta_{2}\right)$ satisfy the constraints imposed by (79) and (80).

It remains to optimize the correlation $\varrho_{\text {sep } 1}\left(\beta_{1}, \beta_{2}\right)$ over $\left(\beta_{1}, \beta_{2}\right)$ subject to the constraints (79) and (80).

$$
\begin{aligned}
\varrho_{\text {sep 1 }}^{*} & =\sup _{\bar{\beta}_{1} \geq \frac{N\left(1-\rho^{2}\right)}{d_{1} P_{1}} 2^{-2 C}, \bar{\beta}_{2} \geq \frac{N\left(1-\rho^{2}\right)}{d_{2} P_{2}}} \sqrt{\beta_{1} \beta_{2}} \\
& =\sqrt{1-\frac{N\left(1-\rho^{2}\right)}{d_{1} P_{1}} 2^{-2 C}} \sqrt{1-\frac{N\left(1-\rho^{2}\right)}{d_{2} P_{2}}} .
\end{aligned}
$$

This concludes the proof of Corollary 5

\section{Proof of Corollary 6}

By Theorem 2, for a fixed conferencing capacity $C_{12}=C$, it follows that any normalized distortion pair $\left(d_{1}, d_{2}\right)$ satisfying $d_{1} \leq 1, d_{2} \leq 1$ and

$$
\begin{aligned}
d_{1} & \geq \frac{N}{\bar{\beta}_{1} P_{1}} 2^{-2 C} \\
d_{2} & \geq \frac{N}{\bar{\beta}_{2} P_{2}} \\
d_{1} d_{2} & =\frac{N\left(1-\rho^{2}\right)\left(1-\check{\rho}^{2}\right)}{P_{1}+P_{2}+2 \sqrt{P_{1} P_{2}}\left(\rho \sqrt{\bar{\beta}_{1} \bar{\beta}_{2}}+\sqrt{\beta_{1} \beta_{2}}\right)}, \quad 0 \leq \beta_{1}, \beta_{2}<1
\end{aligned}
$$

is achievable.

Next, if (27) holds then (83) and (84) are satisfied for sufficiently small $N$ and some $0 \leq$ $\beta_{1}, \beta_{2}<1$. Thus, for $N$ sufficiently small, any pair satisfying (27) and (85), is achievable provided that $\left(\beta_{1}, \beta_{2}\right)$ satisfy the constraints imposed by (83) and (84).

It remains to optimize the correlation $\varrho_{\mathrm{VQ}}\left(\beta_{1}, \beta_{2}\right)$ over $\left(\beta_{1}, \beta_{2}\right)$ subject to the constraints (83) and (84). Instead we compute a lower bound on $\varrho_{\mathrm{VQ}}^{*}$ by evaluating $\varrho_{\mathrm{VQ}}\left(\bar{\beta}_{1}=\frac{N}{d_{1} P_{1}} 2^{-2 C}, \bar{\beta}_{2}=\right.$ $\left.\frac{N}{d_{2} P_{2}}\right)$,

$$
\begin{aligned}
\varrho_{\mathrm{VQ}}^{*} & \triangleq \sup _{\beta_{1}, \beta_{2}}\left(\rho \sqrt{\bar{\beta}_{1} \bar{\beta}_{2}}+\sqrt{\beta_{1} \beta_{2}}\right) \\
& \geq \rho \sqrt{\frac{N}{d_{1} P_{1}} 2^{-2 C}} \sqrt{\frac{N}{d_{2} P_{2}}}+\sqrt{1-\frac{N}{d_{1} P_{1}} 2^{-2 C}} \sqrt{1-\frac{N}{d_{2} P_{2}}}
\end{aligned}
$$

This concludes the proof of Corollary 6

\section{APPENDIX}

The first step in the calculation of the expected distortion of the vector-quantizer scheme is showing that under certain rate constraints the normalized asymptotic distortion of the genieaided scheme is the same as for the originally proposed scheme.

Proposition 6 For every $\delta>0$ and $0<\epsilon<0.3$ there exists an $n^{\prime}(\delta, \epsilon)$ such that for all $n>$ $n^{\prime}(\delta, \epsilon)$,

$$
\frac{1}{n} \mathrm{E}\left[\left\|\mathbf{S}_{\nu}-\hat{\mathbf{S}}_{\nu}\right\|^{2}\right] \leq \frac{1}{n} \mathrm{E}\left[\left\|\mathbf{S}_{\nu}-\hat{\mathbf{S}}_{\nu}^{\mathrm{G}}\right\|^{2}\right]+2 \sigma^{2}(\epsilon+(126 \sqrt{1+\epsilon}+226) \delta) \quad, \quad \nu=1,2
$$


whenever $\left(R_{1}, R_{2}, R_{\mathrm{c}}\right)$ is in the rate region $\mathcal{R}(\epsilon)$ given by

$$
\begin{aligned}
\mathcal{R}(\epsilon)=\left\{\begin{aligned}
R_{1} & \leq \frac{1}{2} \log \left(\frac{\bar{\beta}_{1} P_{1}\left(1-\tilde{\rho}^{2}\right)+N-\bar{\rho}^{2}\left(\bar{\beta}_{1} P_{1}+N\right)}{N\left(1-\tilde{\rho}^{2}\right)-\bar{\rho}^{2} N}-\kappa_{1} \epsilon\right) \\
R_{2} & \leq \frac{1}{2} \log \left(\frac{\bar{\beta}_{2} P_{2}\left(1-\tilde{\rho}^{2}-\bar{\rho}^{2}\right)+N}{N\left(1-\tilde{\rho}^{2}-\bar{\rho}^{2}\right)+\lambda_{2}}-\kappa_{2} \epsilon\right) \\
R_{\mathrm{c}} & \leq \frac{1}{2} \log \left(\frac{\eta^{2}\left(1-\tilde{\rho}^{2}-\bar{\rho}^{2}\right)+N\left(1-\tilde{\rho}^{2}\right)}{N\left(1-\tilde{\rho}^{2}-\bar{\rho}^{2}\right)+\lambda_{c}}-\kappa_{3} \epsilon\right) \\
R_{1}+R_{2} & \leq \frac{1}{2} \log \left(\frac{\lambda_{12}-\bar{\beta}_{2} P_{2} \bar{\rho}^{2}+N}{\left(1-\bar{\beta}_{2} P_{2} \bar{\rho}^{2} \lambda_{12}{ }^{-1}\right) N\left(1-\tilde{\rho}^{2}\right)}-\kappa_{4} \epsilon\right) \\
R_{1}+R_{\mathrm{c}} & \leq \frac{1}{2} \log \left(\frac{\left(\lambda_{1 c}+N\right)\left(\bar{\beta}_{1} P_{1}+\eta^{2}\right)}{\lambda_{1 c} N}-\kappa_{5} \epsilon\right) \\
R_{2}+R_{\mathrm{c}} & \leq \frac{1}{2} \log \left(\frac{\lambda_{2 c}-\bar{\beta}_{2} P_{2} \tilde{\rho}^{2}+N}{\left(1-\bar{\beta}_{2} P_{2} \tilde{\rho}^{2} \lambda_{2 c}{ }^{-1}\right) N\left(1-\bar{\rho}^{2}\right)}-\kappa_{6} \epsilon\right) \\
R_{1}+R_{2}+R_{\mathrm{c}} & \leq \frac{1}{2} \log \left(\frac{\lambda_{12}+2 \eta \bar{\rho} \sqrt{\bar{\beta}_{2} P_{2}}+\eta^{2}+N}{N\left(1-\tilde{\rho}^{2}\right)\left(1-\bar{\rho}^{2}\right)}-\kappa_{7} \epsilon\right) \\
C_{12} & \geq R_{\mathrm{c}}+\frac{1}{2} \log \left(1-\rho^{2} 2^{-2 R_{1}}\left(1-2^{-2 R_{\mathrm{c}}}\right)\right)
\end{aligned}\right\}
\end{aligned}
$$

where $\kappa_{1}, \kappa_{2}, \kappa_{3}, \kappa_{4}, \kappa_{5}, \kappa_{6}$ and $\kappa_{7}$ depend only on $P_{1}, P_{2}, \tilde{\rho}, \bar{\rho}, \beta_{1}, \beta_{2}$, and $N$.

\section{Proof of Proposition 6}

We show that for any $\left(R_{1}, R_{2}, R_{\mathrm{C}}\right) \in \mathcal{R}(\epsilon)$ and sufficiently large $n$, the probability of a decoding error, and consequently $\operatorname{Pr}\left[\left(\hat{\mathbf{S}}_{1}, \hat{\mathbf{S}}_{2}\right) \neq\left(\hat{\mathbf{S}}_{1}^{\mathrm{G}}, \hat{\mathbf{S}}_{2}^{\mathrm{G}}\right)\right]$ is arbitrarily small. To this end, we consider the event consisting of all tuples $\left(\mathbf{s}_{1}, \mathbf{s}_{2}, \mathcal{C}_{1}, \mathcal{C}_{2}, \mathcal{C}_{c}, \mathbf{z}\right)$ for which there exists a triplet $\left(\tilde{\mathbf{u}}_{1}, \tilde{\mathbf{v}}_{\mathbf{v}}, \tilde{\mathbf{u}}_{2}\right) \neq\left(\mathbf{u}_{1}^{*}, \mathbf{v}^{*}, \mathbf{u}_{2}^{*}\right)$ in $\mathcal{C}_{1} \times \mathcal{C}_{c} \times \mathcal{C}_{2}$ that satisfies conditions 60 of the reconstructor, and for which the Euclidean distance between $\mathbf{X}_{\tilde{\mathbf{u}}_{1}, \tilde{\mathbf{v}}, \tilde{\mathbf{u}}_{2}}$ and $\mathbf{y}$ is smaller or equal to the Euclidean distance between $\mathbf{X}_{\mathbf{u}_{1}^{*}, \mathbf{v}^{*}, \mathbf{u}_{2}^{*}}$ and $\mathbf{y}$.

This event is split into seven sub-events:

$$
\mathcal{E}_{\hat{\mathbf{U}}}=\mathcal{E}_{\hat{\mathbf{U}}_{1}} \cup \mathcal{E}_{\hat{\mathbf{U}}_{2}} \cup \mathcal{E}_{\hat{\mathbf{V}}} \cup \mathcal{E}_{\left(\hat{\mathbf{U}}_{1}, \hat{\mathbf{U}}_{2}\right)} \cup \mathcal{E}_{\left(\hat{\mathbf{U}}_{1}, \hat{\mathbf{V}}\right)} \cup \mathcal{E}_{\left(\hat{\mathbf{U}}_{2}, \hat{\mathbf{V}}\right)} \cup \mathcal{E}_{\left(\hat{\mathbf{U}}_{1}, \hat{\mathbf{U}}_{2}, \hat{\mathbf{V}}\right)}
$$

where

$$
\begin{aligned}
& \mathcal{E}_{\hat{\mathbf{U}}_{1}}=\left\{\left(\mathbf{s}_{1}, \mathbf{s}_{2}, \mathcal{C}_{1}, \mathcal{C}_{2}, \mathcal{C}_{c}, \mathbf{z}\right): \exists \tilde{\mathbf{u}}_{1} \in \mathcal{C}_{1} \backslash\left\{\mathbf{u}_{1}^{*}\right\} \text { s.t. }\left|\tilde{\rho}-\cos \varangle\left(\tilde{\mathbf{u}}_{1}, \mathbf{u}_{2}^{*}\right)\right| \leq 7 \epsilon,\right. \\
& \text { and } \left.\left|\cos \varangle\left(\tilde{\mathbf{u}}_{1}, \mathbf{v}^{*}\right)\right| \leq 3 \epsilon \text {, and }\left\|\mathbf{y}-\mathbf{X}_{\tilde{\mathbf{u}}_{1}, \mathbf{v}^{*}, \mathbf{u}_{2}^{*}}\right\|^{2} \leq\left\|\mathbf{y}-\mathbf{X}_{\mathbf{u}_{1}^{*}, \mathbf{v}^{*}, \mathbf{u}_{2}^{*}}\right\|^{2}\right\} \\
& \mathcal{E}_{\hat{\mathbf{U}}_{2}}=\left\{\left(\mathbf{s}_{1}, \mathbf{s}_{2}, \mathcal{C}_{1}, \mathcal{C}_{2}, \mathcal{C}_{c}, \mathbf{z}\right): \exists \tilde{\mathbf{u}}_{2} \in \mathcal{C}_{2} \backslash\left\{\mathbf{u}_{2}^{*}\right\} \text { s.t. }\left|\tilde{\rho}-\cos \varangle\left(\mathbf{u}_{1}^{*}, \tilde{\mathbf{u}}_{2}\right)\right| \leq 7 \epsilon\right. \text {, } \\
& \text { and } \left.\left|\bar{\rho}-\cos \varangle\left(\tilde{\mathbf{u}}_{2}, \mathbf{v}^{*}\right)\right| \leq 7 \epsilon \text {, and }\left\|\mathbf{y}-\mathbf{X}_{\mathbf{u}_{1}^{*}, \mathbf{v}^{*}, \tilde{\mathbf{u}}_{2}}\right\|^{2} \leq\left\|\mathbf{y}-\mathbf{X}_{\mathbf{u}_{1}^{*}, \mathbf{v}^{*}, \mathbf{u}_{2}^{*}}\right\|^{2}\right\} \\
& \mathcal{E}_{\hat{\mathbf{v}}}=\left\{\left(\mathbf{s}_{1}, \mathbf{s}_{2}, \mathcal{C}_{1}, \mathcal{C}_{2}, \mathcal{C}_{c}, \mathbf{z}\right): \exists \tilde{\mathbf{v}} \in \mathcal{C}_{c} \backslash\left\{\mathbf{v}^{*}\right\} \text { s.t. }\left|\cos \varangle\left(\mathbf{u}_{1}^{*}, \tilde{\mathbf{v}}\right)\right| \leq 3 \epsilon,\right. \\
& \text { and } \left.\left|\bar{\rho}-\cos \varangle\left(\mathbf{u}_{2}^{*}, \tilde{\mathbf{v}}\right)\right| \leq 7 \epsilon \text {, and }\left\|\mathbf{y}-\mathbf{X}_{\mathbf{u}_{1}^{*}, \tilde{\mathbf{v}}, \mathbf{u}_{2}^{*}}\right\|^{2} \leq\left\|\mathbf{y}-\mathbf{X}_{\mathbf{u}_{1}^{*}, \mathbf{v}^{*}, \mathbf{u}_{2}^{*}}\right\|^{2}\right\} \\
& \mathcal{E}_{\left(\hat{\mathbf{U}}_{1}, \hat{\mathbf{U}}_{2}\right)}=\left\{\left(\mathbf{s}_{1}, \mathbf{s}_{2}, \mathcal{C}_{1}, \mathcal{C}_{2}, \mathcal{C}_{c}, \mathbf{z}\right): \exists \tilde{\mathbf{u}}_{1} \in \mathcal{C}_{1} \backslash\left\{\mathbf{u}_{1}^{*}\right\} \text { and } \exists \tilde{\mathbf{u}}_{2} \in \mathcal{C}_{2} \backslash\left\{\mathbf{u}_{2}^{*}\right\}\right. \text { s.t. } \\
& \left|\tilde{\rho}-\cos \varangle\left(\tilde{\mathbf{u}}_{1}, \tilde{\mathbf{u}}_{2}\right)\right| \leq 7 \epsilon \text {, and }\left|\cos \varangle\left(\tilde{\mathbf{u}}_{1}, \mathbf{v}^{*}\right)\right| \leq 3 \epsilon \text {, }
\end{aligned}
$$




$$
\begin{aligned}
& \text { and } \left.\left|\bar{\rho}-\cos \varangle\left(\tilde{\mathbf{u}}_{2}, \mathbf{v}^{*}\right)\right| \leq 7 \epsilon \text {, and } \mid \mathbf{y}-\mathbf{X}_{\tilde{\mathbf{u}}_{1}, \mathbf{v}^{*}, \tilde{\mathbf{u}}_{2}}\left\|^{2} \leq\right\| \mathbf{y}-\mathbf{X}_{\mathbf{u}_{1}^{*}, \mathbf{v}^{*}, \mathbf{u}_{2}^{*}} \|^{2}\right\} \\
& \mathcal{E}_{\left(\hat{\mathbf{U}}_{1}, \hat{\mathbf{v}}\right)}=\left\{\left(\mathbf{s}_{1}, \mathbf{s}_{2}, \mathcal{C}_{1}, \mathcal{C}_{2}, \mathcal{C}_{c}, \mathbf{z}\right): \exists \tilde{\mathbf{u}}_{1} \in \mathcal{C}_{1} \backslash\left\{\mathbf{u}_{1}^{*}\right\} \text { and } \exists \tilde{\mathbf{v}} \in \mathcal{C}_{c} \backslash\left\{\mathbf{v}^{*}\right\}\right. \text { s.t. } \\
& \left|\tilde{\rho}-\cos \varangle\left(\tilde{\mathbf{u}}_{1}, \mathbf{u}_{2}^{*}\right)\right| \leq 7 \epsilon \text {, and }\left|\cos \varangle\left(\tilde{\mathbf{u}}_{1}, \tilde{\mathbf{v}}\right)\right| \leq 3 \epsilon \text {, } \\
& \text { and } \left.\left|\bar{\rho}-\cos \varangle\left(\mathbf{u}_{2}^{*}, \tilde{\mathbf{v}}\right)\right| \leq 7 \epsilon \text {, and } \mid \mathbf{y}-\mathbf{X}_{\tilde{\mathbf{u}}_{1}, \tilde{\mathbf{v}}, \mathbf{u}_{2}^{*}}\left\|^{2} \leq\right\| \mathbf{y}-\mathbf{X}_{\mathbf{u}_{1}^{*}, \mathbf{v}^{*}, \mathbf{u}_{2}^{*}} \|^{2}\right\} \\
& \mathcal{E}_{\left(\hat{\mathbf{U}}_{2}, \hat{\mathbf{v}}\right)}=\left\{\left(\mathbf{s}_{1}, \mathbf{s}_{2}, \mathcal{C}_{1}, \mathcal{C}_{2}, \mathcal{C}_{c}, \mathbf{z}\right): \exists \tilde{\mathbf{u}}_{2} \in \mathcal{C}_{2} \backslash\left\{\mathbf{u}_{2}^{*}\right\} \text { and } \exists \tilde{\mathbf{v}} \in \mathcal{C}_{c} \backslash\left\{\mathbf{v}^{*}\right\}\right. \text { s.t. } \\
& \left|\tilde{\rho}-\cos \varangle\left(\mathbf{u}_{1}^{*}, \tilde{\mathbf{u}}_{2}\right)\right| \leq 7 \epsilon \text {, and }\left|\cos \varangle\left(\mathbf{u}_{1}^{*}, \tilde{\mathbf{v}}\right)\right| \leq 3 \epsilon \text {, } \\
& \text { and } \left.\left|\bar{\rho}-\cos \varangle\left(\tilde{\mathbf{u}}_{2}, \tilde{\mathbf{v}}\right)\right| \leq 7 \epsilon \text {, and } \mid \mathbf{y}-\mathbf{X}_{\mathbf{u}_{1}^{*}, \tilde{\mathbf{v}}, \tilde{\mathbf{u}}_{2}}\left\|^{2} \leq\right\| \mathbf{y}-\mathbf{X}_{\mathbf{u}_{1}^{*}, \mathbf{v}^{*}, \mathbf{u}_{2}^{*}} \|^{2}\right\} \\
& \mathcal{E}_{\left(\hat{\mathbf{U}}_{1}, \hat{\mathbf{U}}_{2}, \hat{\mathbf{v}}\right)}=\left\{\left(\mathbf{s}_{1}, \mathbf{s}_{2}, \mathcal{C}_{1}, \mathcal{C}_{2}, \mathcal{C}_{c}, \mathbf{z}\right): \exists \tilde{\mathbf{u}}_{1} \in \mathcal{C}_{1} \backslash\left\{\mathbf{u}_{1}^{*}\right\} \text { and } \exists \tilde{\mathbf{u}}_{2} \in \mathcal{C}_{2} \backslash\left\{\mathbf{u}_{2}^{*}\right\} \text { and } \exists \tilde{\mathbf{v}} \in \mathcal{C}_{c} \backslash\left\{\mathbf{v}^{*}\right\}\right. \\
& \text { s.t. }\left|\tilde{\rho}-\cos \varangle\left(\tilde{\mathbf{u}}_{1}, \tilde{\mathbf{u}}_{2}\right)\right| \leq 7 \epsilon \text {, and }\left|\cos \varangle\left(\tilde{\mathbf{u}}_{1}, \tilde{\mathbf{v}}\right)\right| \leq 3 \epsilon \text {, } \\
& \text { and } \left.\left|\bar{\rho}-\cos \varangle\left(\tilde{\mathbf{u}}_{2}, \tilde{\mathbf{v}}\right)\right| \leq 7 \epsilon \text {, and } \mid \mathbf{y}-\mathbf{X}_{\tilde{\mathbf{u}}_{1}, \tilde{\mathbf{v}}, \tilde{\mathbf{u}}_{2}}\left\|^{2} \leq\right\| \mathbf{y}-\mathbf{X}_{\mathbf{u}_{1}^{*}, \mathbf{v}^{*}, \mathbf{u}_{2}^{*}} \|^{2}\right\} \text {, }
\end{aligned}
$$

where $\mathbf{y} \triangleq a_{1,1} \mathbf{u}_{1}^{*}+a_{2,1} \mathbf{u}_{2}^{*}+\alpha \mathbf{v}^{*}+\mathbf{z}$.

Note that a decoding error occurs only if $\left(\mathbf{s}_{1}, \mathbf{s}_{2}, \mathcal{C}_{1}, \mathcal{C}_{2}, \mathcal{C}_{c}, \mathbf{z}\right) \in \mathcal{E}_{\hat{\mathbf{U}}}$.

The main result of this section can now be stated as follows:

Lemma 5 For every $\delta>0$ and $0.3>\epsilon>0$, there exists an $n_{4}^{\prime}(\delta, \epsilon)$ such that for all $n>$ $n_{4}^{\prime}(\delta, \epsilon)$

$$
\operatorname{Pr}\left[\mathcal{E}_{\hat{\mathbf{U}}}\right]<21 \delta, \quad \text { whenever }\left(R_{1}, R_{2}, R_{\mathrm{c}}\right) \in \mathcal{R}(\epsilon) .
$$

To prove Lemma [5 we introduce three auxiliary error events. The first auxiliary event $\mathcal{E}_{\mathbf{S}}$ corresponds to an atypical source output. More precisely,

$$
\begin{aligned}
\mathcal{E}_{\mathbf{S}}=\left\{\left(\mathbf{s}_{1}, \mathbf{s}_{2}\right) \in \mathbb{R}^{n} \times \mathbb{R}^{n}:\left|\frac{1}{n}\left\|\mathbf{s}_{1}\right\|^{2}-\sigma^{2}\right|>\epsilon \sigma^{2}\right. \\
\left.\quad \text { or }\left|\frac{1}{n}\left\|\mathbf{s}_{2}\right\|^{2}-\sigma^{2}\right|>\epsilon \sigma^{2} \text { or }\left|\cos \varangle\left(\mathbf{s}_{1}, \mathbf{s}_{2}\right)-\rho\right|>\epsilon \rho\right\} .
\end{aligned}
$$

The second auxiliary event is denoted by $\mathcal{E}_{\mathbf{Z}}$ and corresponds to an atypical behavior of the additive noise:

$$
\begin{aligned}
\mathcal{E}_{\mathbf{Z}}=\left\{\left(\mathbf{s}_{1}, \mathbf{s}_{2}, \mathcal{C}_{1}, \mathcal{C}_{2}, \mathcal{C}_{c}, \mathbf{z}\right):\right. & \frac{1}{n}\|\mathbf{z}\|^{2}-N \mid>\epsilon N \\
& \text { or } \frac{1}{n}\left|\left\langle a_{1,1} \mathbf{u}_{1}^{*}\left(\mathbf{s}_{1}, \mathcal{C}_{1}\right), \mathbf{z}\right\rangle\right|>\sqrt{\bar{\beta}_{1} P_{1} N} \epsilon \text { or } \frac{1}{n}\left|\left\langle a_{2,1} \mathbf{u}_{2}^{*}\left(\mathbf{s}_{2}, \mathcal{C}_{2}\right), \mathbf{z}\right\rangle\right|>\sqrt{\bar{\beta}_{2} P_{2} N \epsilon} \\
& \text { or } \left.\frac{1}{n}\left|\left\langle\alpha \mathbf{v}^{*}\left(\mathbf{s}_{1}, \mathcal{C}_{1}, \mathcal{C}_{c}\right), \mathbf{z}\right\rangle\right|>\frac{1}{n}\left\|\alpha \mathbf{v}^{*}\left(\mathbf{s}_{1}, \mathcal{C}_{1}, \mathcal{C}_{c}\right)\right\| \sqrt{n N} \epsilon\right\} .
\end{aligned}
$$

Finally, the third auxiliary event is denoted by $\mathcal{E}_{\mathbf{X}}$ and corresponds to irregularities at the encoders. That is, the event that one of the codebooks contains no codeword satisfying Condition (57) or condition (59) of the vector-quantizer, or that the quantized sequences $\mathbf{u}_{1}^{*}$ and $\mathbf{u}_{2}^{*}$ and $\mathbf{v}^{*}$ have an atypical angle to each other, or that Encoder 2 recovers a codeword $\tilde{\mathbf{v}} \neq \mathbf{v}^{*}$. More formally, $\mathcal{E}_{\mathbf{X}}=\mathcal{E}_{\mathbf{X}_{1}} \cup \mathcal{E}_{\mathbf{X}_{2}} \cup \mathcal{E}_{\mathbf{X}_{v}} \cup \mathcal{E}_{\left(\mathbf{X}_{1}, \mathbf{X}_{2}\right)} \cup \mathcal{E}_{\left(\mathbf{X}_{1}, \mathbf{X}_{v}\right)} \cup \mathcal{E}_{\left(\mathbf{X}_{2}, \mathbf{X}_{v}\right)} \cup \mathcal{E}_{\mathbf{X}_{\mathrm{WZ}}}$ where

$$
\begin{aligned}
& \mathcal{E}_{\mathbf{X}_{1}}=\left\{\left(\mathbf{s}_{1}, \mathbf{s}_{2}, \mathcal{C}_{1}, \mathcal{C}_{2}, \mathcal{C}_{c}\right): \nexists \mathbf{u}_{1} \in \mathcal{C}_{1}\right. \text { s.t. } \\
&\left.\left|\sqrt{1-2^{-2 R_{1}}}-\cos \varangle\left(\mathbf{s}_{1}, \mathbf{u}_{1}\right)\right| \leq \epsilon \sqrt{1-2^{-2 R_{1}}}\right\} \\
& \mathcal{E}_{\mathbf{X}_{2}}=\left\{\left(\mathbf{s}_{1}, \mathbf{s}_{2}, \mathcal{C}_{1}, \mathcal{C}_{2}, \mathcal{C}_{c}\right): \nexists \mathbf{u}_{2} \in \mathcal{C}_{2}\right. \text { s.t. }
\end{aligned}
$$




$$
\begin{aligned}
& \left.\left|\sqrt{1-2^{-2 R_{2}}}-\cos \varangle\left(\mathbf{s}_{2}, \mathbf{u}_{2}\right)\right| \leq \epsilon \sqrt{1-2^{-2 R_{2}}}\right\} \\
& \mathcal{E}_{\mathbf{X}_{v}}=\left\{\left(\mathbf{s}_{1}, \mathbf{s}_{2}, \mathcal{C}_{1}, \mathcal{C}_{2}, \mathcal{C}_{c}\right): \nexists \mathbf{v} \in \mathcal{C}_{c}\right. \text { s.t. } \\
& \left.\left|\sqrt{1-2^{-2 R_{\mathrm{c}}}}-\cos \varangle\left(\mathbf{z}_{Q_{1}}, \mathbf{v}\right)\right| \leq \epsilon \sqrt{1-2^{-2 R_{\mathrm{c}}}}\right\} \\
& \mathcal{E}_{\left(\mathbf{x}_{1}, \mathbf{X}_{2}\right)}=\left\{\left(\mathbf{s}_{1}, \mathbf{s}_{2}, \mathcal{C}_{1}, \mathcal{C}_{2}, \mathcal{C}_{c}\right):\left|\tilde{\rho}-\cos \varangle\left(\mathbf{u}_{1}^{*}, \mathbf{u}_{2}^{*}\right)\right|>7 \epsilon\right\} \\
& \mathcal{E}_{\left(\mathbf{x}_{1}, \mathbf{x}_{v}\right)}=\left\{\left(\mathbf{s}_{1}, \mathbf{s}_{2}, \mathcal{C}_{1}, \mathcal{C}_{2}, \mathcal{C}_{c}\right):\left|\cos \varangle\left(\mathbf{u}_{1}^{*}, \mathbf{v}^{*}\right)\right|>3 \epsilon\right\} \\
& \mathcal{E}_{\left(\mathbf{x}_{2}, \mathbf{X}_{v}\right)}=\left\{\left(\mathbf{s}_{1}, \mathbf{s}_{2}, \mathcal{C}_{1}, \mathcal{C}_{2}, \mathcal{C}_{c}\right):\left|\bar{\rho}-\cos \varangle\left(\mathbf{v}^{*}, \mathbf{u}_{2}^{*}\right)\right|>7 \epsilon\right\} \\
& \mathcal{E}_{\mathbf{X}_{\mathrm{wZ}}}=\left\{\left(\mathbf{s}_{1}, \mathbf{s}_{2}, \mathcal{C}_{1}, \mathcal{C}_{2}, \mathcal{C}_{c}\right): \exists \tilde{\mathbf{v}} \in \mathcal{C}_{c} \backslash\left\{\mathbf{v}^{*}\right\} \text { s.t. }\left|\rho_{\mathbf{v}, \mathbf{s}_{2}}-\cos \varangle\left(\tilde{\mathbf{v}}, \mathbf{s}_{2}\right)\right| \leq 5 \epsilon\right\} .
\end{aligned}
$$

To prove Lemma [5] we now start with the decomposition

$$
\begin{aligned}
\operatorname{Pr}\left[\mathcal{E}_{\hat{\mathbf{U}}}\right]= & \operatorname{Pr}\left[\mathcal{E}_{\hat{\mathbf{U}}} \cap \mathcal{E}_{\mathbf{S}}^{c} \cap \mathcal{E}_{\mathbf{X}}^{c} \cap \mathcal{E}_{\mathbf{Z}}^{c}\right]+\operatorname{Pr}\left[\mathcal{E}_{\hat{\mathbf{U}}} \mid \mathcal{E}_{\mathbf{S}} \cup \mathcal{E}_{\mathbf{X}} \cup \mathcal{E}_{\mathbf{Z}}\right] \operatorname{Pr}\left[\mathcal{E}_{\mathbf{S}} \cup \mathcal{E}_{\mathbf{X}} \cup \mathcal{E}_{\mathbf{Z}}\right] \\
\leq & \operatorname{Pr}\left[\mathcal{E}_{\hat{\mathbf{U}}} \cap \mathcal{E}_{\mathbf{S}}^{c} \cap \mathcal{E}_{\mathbf{X}}^{c} \cap \mathcal{E}_{\mathbf{Z}}^{c}\right]+\operatorname{Pr}\left[\mathcal{E}_{\mathbf{S}}\right]+\operatorname{Pr}\left[\mathcal{E}_{\mathbf{X}}\right]+\operatorname{Pr}\left[\mathcal{E}_{\mathbf{Z}}\right] \\
\leq & \operatorname{Pr}\left[\mathcal{E}_{\hat{\mathbf{U}}_{1}} \cap \mathcal{E}_{\mathbf{S}}^{c} \cap \mathcal{E}_{\mathbf{X}}^{c} \cap \mathcal{E}_{\mathbf{Z}}^{c}\right]+\operatorname{Pr}\left[\mathcal{E}_{\hat{\mathbf{U}}_{2}} \cap \mathcal{E}_{\mathbf{S}}^{c} \cap \mathcal{E}_{\mathbf{X}}^{c} \cap \mathcal{E}_{\mathbf{Z}}^{c}\right]+\operatorname{Pr}\left[\mathcal{E}_{\hat{\mathbf{V}}} \cap \mathcal{E}_{\mathbf{S}}^{c} \cap \mathcal{E}_{\mathbf{X}}^{c} \cap \mathcal{E}_{\mathbf{Z}}^{c}\right] \\
& +\operatorname{Pr}\left[\mathcal{E}_{\left(\hat{\mathbf{U}}_{1}, \hat{\mathbf{U}}_{2}\right)} \cap \mathcal{E}_{\mathbf{S}}^{c} \cap \mathcal{E}_{\mathbf{X}}^{c} \cap \mathcal{E}_{\mathbf{Z}}^{c}\right]+\operatorname{Pr}\left[\mathcal{E}_{\left(\hat{\mathbf{U}}_{1}, \hat{\mathbf{V}}\right)} \cap \mathcal{E}_{\mathbf{S}}^{c} \cap \mathcal{E}_{\mathbf{X}}^{c} \cap \mathcal{E}_{\mathbf{Z}}^{c}\right] \\
& +\operatorname{Pr}\left[\mathcal{E}_{\left(\hat{\mathbf{U}}_{2}, \hat{\mathbf{V}}\right)} \cap \mathcal{E}_{\mathbf{S}}^{c} \cap \mathcal{E}_{\mathbf{X}}^{c} \cap \mathcal{E}_{\mathbf{Z}}^{c}\right]+\operatorname{Pr}\left[\mathcal{E}_{\left(\hat{\mathbf{U}}_{1}, \hat{\mathbf{U}}_{2}, \hat{\mathbf{V}}\right)} \cap \mathcal{E}_{\mathbf{S}}^{c} \cap \mathcal{E}_{\mathbf{X}}^{c} \cap \mathcal{E}_{\mathbf{Z}}^{c}\right]+\operatorname{Pr}\left[\mathcal{E}_{\mathbf{S}}\right] \\
& +\operatorname{Pr}\left[\mathcal{E}_{\mathbf{X}}\right]+\operatorname{Pr}\left[\mathcal{E}_{\mathbf{Z}}\right],
\end{aligned}
$$

where we have used the shorthand notation $\operatorname{Pr}\left[\mathcal{E}_{\nu}\right]$ for the probability

$\operatorname{Pr}\left[\left(\mathbf{s}_{1}, \mathbf{s}_{2}, \mathcal{C}_{1}, \mathcal{C}_{2}, \mathcal{C}_{c}, \mathbf{z}\right) \in \mathcal{E}_{\nu}\right]$, and where $\mathcal{E}_{\nu}^{c}$ denotes the complement of $\mathcal{E}_{\nu}$. Lemma 5 now follows from upper-bounding the probability terms on the r.h.s. of (101).

Lemma 6 For every $\delta>0$ and $\epsilon>0$ there exists an $n^{\prime}(\delta, \epsilon) \in \mathbb{N}$ such that for all $n>n^{\prime}(\delta, \epsilon)$

$$
\operatorname{Pr}\left[\mathcal{E}_{\mathbf{S}}\right]<\delta .
$$

Proof: The proof follows by the weak law of large numbers.

Lemma 7 For every $\delta>0$ and $\epsilon>0$ there exists an $n^{\prime}(\delta, \epsilon) \in \mathbb{N}$ such that for all $n>n^{\prime}(\delta, \epsilon)$

$$
\operatorname{Pr}\left[\mathcal{E}_{\mathbf{Z}}\right]<\delta \text {. }
$$

Proof: The proof follows by the weak law of large numbers, and since for every $\epsilon>0$

$$
\sup _{\| \mathbf{u} \in \mathbb{R}^{n}:} \operatorname{Pr}\left[\frac{1}{n}\left\langle a_{i, 1} \mathbf{u}, \mathbf{z}\right\rangle>\sqrt{P_{i} N} \epsilon\right] \rightarrow 0 \quad \text { as } n \rightarrow \infty,
$$

where $i \in\{1,2\}$. The same argument holds for $\mathbf{v}$.

Lemma 8 For every $\delta>0$ and $1>\epsilon>0$ there exists an $n^{\prime}(\delta, \epsilon) \in \mathbb{N}$ such that for all $n>n^{\prime}(\delta, \epsilon)$

$$
\operatorname{Pr}\left[\mathcal{E}_{\mathbf{X}}\right]<12 \delta .
$$

Proof: This result follows from rate-distortion theory. The detailed proof for our setting is given in Section $\mathrm{H}$ of the Appendix. 
Lemma 9 For every $\delta>0$ and $\epsilon>0$ there exists an $n_{4}^{\prime \prime}(\delta, \epsilon) \in \mathbb{N}$ such that for all $n>n_{4}^{\prime \prime}(\delta, \epsilon)$

$$
\begin{aligned}
& \operatorname{Pr} {\left[\mathcal{E}_{\hat{\mathbf{U}}_{1}} \cap \mathcal{E}_{\mathbf{S}}^{c} \cap \mathcal{E}_{\mathbf{X}}^{c} \cap \mathcal{E}_{\mathbf{Z}}^{c}\right] \leq \delta, } \\
& \text { if } R_{1}<\frac{1}{2} \log \left(\frac{\bar{\beta}_{1} P_{1}\left(1-\tilde{\rho}^{2}\right)+N-\bar{\rho}^{2}\left(\bar{\beta}_{1} P_{1}+N\right)}{N\left(1-\tilde{\rho}^{2}\right)-\bar{\rho}^{2} N}-\kappa_{1} \epsilon\right) \\
& \operatorname{Pr} {\left[\mathcal{E}_{\hat{\mathbf{U}}_{2}} \cap \mathcal{E}_{\mathbf{S}}^{c} \cap \mathcal{E}_{\mathbf{X}}^{c} \cap \mathcal{E}_{\mathbf{Z}}^{c}\right] \leq \delta, } \\
& \text { if } R_{2}<\frac{1}{2} \log \left(\frac{\bar{\beta}_{2} P_{2}\left(1-\tilde{\rho}^{2}-\bar{\rho}^{2}\right)+N}{N\left(1-\tilde{\rho}^{2}-\bar{\rho}^{2}\right)+\lambda_{2}}-\kappa_{2} \epsilon\right) \\
& \operatorname{Pr} {\left[\mathcal{E}_{\left.\hat{\mathbf{V}} \cap \mathcal{E}_{\mathbf{S}}^{c} \cap \mathcal{E}_{\mathbf{X}}^{c} \cap \mathcal{E}_{\mathbf{Z}}^{c}\right] \leq \delta,}\right.} \\
& \text { if } R_{\mathbf{c}}<\frac{1}{2} \log \left(\frac{\eta^{2}\left(1-\tilde{\rho}^{2}-\bar{\rho}^{2}\right)+N\left(1-\tilde{\rho}^{2}\right)}{N\left(1-\tilde{\rho}^{2}-\bar{\rho}^{2}\right)+\lambda_{c}}-\kappa_{3} \epsilon\right) \\
& \operatorname{Pr}\left[\mathcal{E}_{\left(\hat{\mathbf{U}}_{1}, \hat{\mathbf{U}}_{2}\right)} \cap \mathcal{E}_{\mathbf{S}}^{c} \cap \mathcal{E}_{\mathbf{X}}^{c} \cap \mathcal{E}_{\mathbf{Z}}^{c}\right] \leq \delta, \\
& \text { if } R_{1}+R_{2}<\frac{1}{2} \log \left(\frac{\lambda_{12}-\bar{\beta}_{2} P_{2} \bar{\rho}^{2}+N}{\left(1-\bar{\beta}_{2} P_{2} \bar{\rho}^{2} \lambda_{12}{ }^{-1}\right) N\left(1-\tilde{\rho}^{2}\right)}-\kappa_{4} \epsilon\right) \\
& \operatorname{Pr}\left[\mathcal{E}_{\left(\hat{\mathbf{U}}_{1}, \hat{\mathbf{V}}\right)} \cap \mathcal{E}_{\mathbf{S}}^{c} \cap \mathcal{E}_{\mathbf{X}}^{c} \cap \mathcal{E}_{\mathbf{Z}}^{c}\right] \leq \delta, \\
& \text { if } R_{1}+R_{\mathrm{c}}<\frac{1}{2} \log \left(\frac{\left(\lambda_{1 c}+N\right)\left(\bar{\beta}_{1} P_{1}+\eta^{2}\right)}{\lambda_{1 c} N}-\kappa_{5} \epsilon\right) \\
& \operatorname{Pr}\left[\mathcal{E}_{\left(\hat{\mathbf{U}}_{2}, \hat{\mathbf{V}}\right)} \cap \mathcal{E}_{\mathbf{S}}^{c} \cap \mathcal{E}_{\mathbf{X}}^{c} \cap \mathcal{E}_{\mathbf{Z}}^{c}\right] \leq \delta, \\
& \text { if } R_{2}+R_{\mathrm{c}}<\frac{1}{2} \log \left(\frac{\lambda_{2 c}-\bar{\beta}_{2} P_{2} \tilde{\rho}^{2}+N}{\left(1-\bar{\beta}_{2} P_{2} \tilde{\rho}^{2} \lambda_{2 c}{ }^{-1}\right) N\left(1-\bar{\rho}^{2}\right)}-\kappa_{6} \epsilon\right) \\
& \operatorname{Pr}\left[\mathcal{E}_{\left(\hat{\mathbf{U}}_{1}, \hat{\mathbf{U}}_{2}, \hat{\mathbf{V}}\right)} \cap \mathcal{E}_{\mathbf{S}}^{c} \cap \mathcal{E}_{\mathbf{X}}^{c} \cap \mathcal{E}_{\mathbf{Z}}^{c}\right] \leq \delta, \\
& \text { if } R_{1}+R_{2}+R_{\mathrm{c}}<\frac{1}{2} \log \left(\frac{\lambda_{12}+2 \eta \bar{\rho} \sqrt{\beta_{2} P_{2}}+\eta^{2}+N}{N\left(1-\tilde{\rho}^{2}\right)\left(1-\bar{\rho}^{2}\right)}-\kappa_{7} \epsilon\right),
\end{aligned}
$$

where $\kappa_{1}, \kappa_{2}, \kappa_{3}, \kappa_{4}, \kappa_{5}, \kappa_{6}$ and $\kappa_{7}$ are positive constants determined by $P_{1}, P_{2}$, and $N$.

The proof of this lemma appears in subsections $A-G$ of the Appendix.

\section{Concluding the proof of Proposition 6}

We start with five lemmas. The first lemma upper bounds the impact of atypical source outputs on the expected distortion.

Lemma 10 For every $\epsilon>0$

$$
\frac{1}{n} \mathrm{E}\left[\left\|\mathbf{S}_{1}\right\|^{2} \mid \mathcal{E}_{\mathbf{S}}\right] \operatorname{Pr}\left[\mathcal{E}_{\mathbf{S}}\right] \leq \sigma^{2}\left(\epsilon+\operatorname{Pr}\left[\mathcal{E}_{\mathbf{S}}\right]\right) .
$$

Proof:

$$
\begin{aligned}
\frac{1}{n} \mathrm{E}\left[\left\|\mathbf{S}_{1}\right\|^{2} \mid \mathcal{E}_{\mathbf{S}}\right] \operatorname{Pr}\left[\mathcal{E}_{\mathbf{S}}\right] & =\frac{1}{n} \mathrm{E}\left[\left\|\mathbf{S}_{1}\right\|^{2}\right]-\frac{1}{n} \mathrm{E}\left[\left\|\mathbf{S}_{1}\right\|^{2} \mid \mathcal{E}_{\mathbf{S}}^{c}\right] \operatorname{Pr}\left[\mathcal{E}_{\mathbf{S}}^{c}\right] \\
& \leq \sigma^{2}-\sigma^{2}(1-\epsilon) \operatorname{Pr}\left[\mathcal{E}_{\mathbf{S}}^{c}\right] \\
& =\sigma^{2}-\sigma^{2}(1-\epsilon)\left(1-\operatorname{Pr}\left[\mathcal{E}_{\mathbf{S}}\right]\right)
\end{aligned}
$$




$$
\begin{aligned}
& =\sigma^{2} \epsilon+\sigma^{2}(1-\epsilon) \operatorname{Pr}\left[\mathcal{E}_{\mathbf{S}}\right] \\
& \leq \sigma^{2}\left(\epsilon+\operatorname{Pr}\left[\mathcal{E}_{\mathbf{S}}\right]\right)
\end{aligned}
$$

The second lemma considers the properties of the estimator coefficients.

Lemma 11 The gain coefficients in (15) satisfy $\gamma_{1,1}, \gamma_{1,3}, \gamma_{2,2} \leq 1$ and $\gamma_{1,2}, \gamma_{2,1}, \gamma_{2,3} \leq \rho$.

Proof: The first claim is obvious, so we will first show that $\gamma_{1,2} \leq \rho$. Note that

$$
\begin{aligned}
\gamma_{1,2} & =\frac{\rho 2^{-2\left(R_{1}+R_{\mathrm{c}}\right)}}{1-\rho^{2}\left(1-2^{-2 R_{2}}\right)\left(1-2^{-2\left(R_{1}+R_{\mathrm{c}}\right)}\right)} \\
& =\frac{\rho}{2^{2\left(R_{1}+R_{\mathrm{c}}\right)}-\rho^{2}\left(1-2^{-2 R_{2}}\right)\left(2^{2\left(R_{1}+R_{\mathrm{c}}\right)}-1\right)} \\
& =\frac{\rho}{2^{2\left(R_{1}+R_{\mathrm{c}}\right)}\left(1-\rho^{2}\left(1-2^{-2 R_{2}}\right)\right)+\rho^{2}\left(1-2^{-2 R_{2}}\right)} .
\end{aligned}
$$

Now, consider the function

$$
f(\alpha, \beta)=\frac{1}{\beta(1-\alpha)+\alpha},
$$

where $0<\alpha<1$ and $\beta \geq 1$. Note that $f(\alpha, 1)=1$, and that $\frac{\partial f(\alpha, \beta)}{\partial \beta}=-\frac{(1-\alpha)}{(\beta(1-\alpha)+\alpha)^{2}}<0$. Thus $f(\alpha, \beta)$ is continuous and monotonically decreasing in $\beta$ for $\beta \geq 1$.

On the other hand, note that $f(0, \beta)=\frac{1}{\beta}<1$ assuming $\beta>1$, and $f(1, \beta)=1$ and $\frac{\partial f(\alpha, \beta)}{\partial \alpha}=$ $-\frac{(1-\beta)}{(\beta(1-\alpha)+\alpha)^{2}}>0$. Thus, $f(\alpha, \beta)$ is continuous and monotonically increasing in $\alpha$ for $0<\alpha<$ 1 , and therefore $0<f(\alpha, \beta) \leq 1$ for $0<\alpha<1$ and $\beta>1$.

The proof that $\gamma_{2,1}, \gamma_{2,3} \leq \rho$ follows in a similar way.

The third lemma gives upper bounds on norms related to the reconstructions $\hat{\mathbf{s}}_{1}$ and $\hat{\mathbf{s}}_{1}^{\mathrm{G}}$.

Lemma 12 Let the reconstructions $\hat{\mathbf{s}}_{1}$ and $\hat{\mathbf{s}}_{1}^{\mathrm{G}}$ be as defined in (14) and (62). Then,

$$
\left\|\hat{\mathbf{s}}_{1}\right\|^{2} \leq 9 n \sigma^{2}, \quad\left\|\hat{\mathbf{s}}_{1}^{\mathrm{G}}\right\|^{2} \leq 9 n \sigma^{2}, \quad\left\|\hat{\mathbf{s}}_{1}^{\mathrm{G}}-\hat{\mathbf{s}}_{1}\right\|^{2} \leq 36 n \sigma^{2} .
$$

Proof: We start by upper-bounding the squared norm of $\hat{\mathbf{s}}_{1}$

$$
\begin{aligned}
\left\|\hat{\mathbf{s}}_{1}\right\|^{2}= & \left\|\gamma_{1,1} \hat{\mathbf{u}}_{1}+\gamma_{1,2} \hat{\mathbf{u}}_{2}+\gamma_{1,3} \hat{\mathbf{v}}\right\|^{2} \\
= & \gamma_{1,1}^{2}\left\|\hat{\mathbf{u}}_{1}\right\|^{2}+2 \gamma_{1,1} \gamma_{1,2}\left\langle\hat{\mathbf{u}}_{1}, \hat{\mathbf{u}}_{2}\right\rangle+\gamma_{1,2}^{2}\left\|\hat{\mathbf{u}}_{2}\right\|^{2}+2 \gamma_{1,1} \gamma_{1,3}\left\langle\hat{\mathbf{u}}_{1}, \hat{\mathbf{v}}\right\rangle+2 \gamma_{1,2} \gamma_{1,3}\left\langle\hat{\mathbf{u}}_{2}, \hat{\mathbf{v}}\right\rangle \\
& +\gamma_{1,3}^{2}\left\|\hat{\mathbf{v}}^{2}\right\|^{2} \\
\leq & \gamma_{1,1}^{2}\left\|\hat{\mathbf{u}}_{1}\right\|^{2}+2 \gamma_{1,1} \gamma_{1,2}\left\|\hat{\mathbf{u}}_{1}\right\|\left\|\hat{\mathbf{u}}_{2}\right\|+\gamma_{1,2}^{2}\left\|\hat{\mathbf{u}}_{2}\right\|^{2}+2 \gamma_{1,1} \gamma_{1,3}\left\|\hat{\mathbf{u}}_{1}\right\|\|\hat{\mathbf{v}}\| \\
& +2 \gamma_{1,2} \gamma_{1,3}\left\|\hat{\mathbf{u}}_{2}\right\|\|\hat{\mathbf{v}}\|+\gamma_{1,3}^{2}\|\hat{\mathbf{v}}\|^{2} \\
= & \left(\gamma_{1,1}\left\|\hat{\mathbf{u}}_{1}\right\|+\gamma_{1,2}\left\|\hat{\mathbf{u}}_{2}\right\|+\gamma_{1,3}\|\hat{\mathbf{v}}\|\right)^{2} \\
& (a) \\
\leq & n \sigma^{2}(2+\rho)^{2} \\
\leq & 9 n \sigma^{2},
\end{aligned}
$$

where in (a) we have used Lemma 11 i.e. that $\gamma_{1,1}, \gamma_{1,3} \leq 1$ and $\gamma_{1,2} \leq \rho$, and that $\left\|\hat{\mathbf{u}}_{i}\right\| \leq$ $\sqrt{n \sigma^{2}}, i \in\{1,2\}$ and $\|\hat{\mathbf{v}}\| \leq \sqrt{n \sigma^{2}}$. The upper bound on the squared norm of $\hat{\mathbf{s}}_{1}^{\mathrm{G}}$ is obtained similarly. Its proof is therefore omitted. The upper bound on the squared norm of the difference 
between $\hat{\mathbf{s}}_{1}$ and $\hat{\mathbf{s}}_{1}^{\mathrm{G}}$ now follows easily:

$$
\begin{aligned}
\left\|\hat{\mathbf{s}}_{1}^{\mathrm{G}}-\hat{\mathbf{s}}_{1}\right\|^{2} & \leq\left\|\hat{\mathbf{s}}_{1}^{\mathrm{G}}\right\|^{2}+2\left\|\hat{\mathbf{s}}_{1}^{\mathrm{G}}\right\|\left\|\hat{\mathbf{s}}_{1}\right\|+\left\|\hat{\mathbf{s}}_{1}\right\|^{2} \\
& =\left(\left\|\hat{\mathbf{s}}_{1}^{\mathrm{G}}\right\|+\left\|\hat{\mathbf{s}}_{1}\right\|\right)^{2} \\
& \leq 36 n \sigma^{2}
\end{aligned}
$$

The next two lemmas are used directly in the upcoming proof of Proposition 6 They rely on Lemma 10 and Lemma 12

\section{Lemma 13}

$$
\frac{1}{n} \mathrm{E}\left[\left\langle\mathbf{S}_{1}, \hat{\mathbf{S}}_{1}^{G}-\hat{\mathbf{S}}_{1}\right\rangle\right] \leq \sigma^{2}\left(\epsilon+37 \operatorname{Pr}\left[\mathcal{E}_{\mathbf{S}}\right]+6 \sqrt{1+\epsilon} \operatorname{Pr}\left[\mathcal{E}_{\hat{\mathbf{U}}}\right]\right) .
$$

Proof:

$$
\begin{aligned}
& \frac{1}{n} \mathrm{E}\left[\left\langle\mathbf{S}_{1}, \hat{\mathbf{S}}_{1}^{\mathrm{G}}-\hat{\mathbf{S}}_{1}\right\rangle\right]=\frac{1}{n} \mathrm{E}\left[\left\langle\mathbf{S}_{1}, \hat{\mathbf{S}}_{1}^{\mathrm{G}}-\hat{\mathbf{S}}_{1}\right\rangle \mid \mathcal{E}_{\mathbf{S}}\right] \operatorname{Pr}\left[\mathcal{E}_{\mathbf{S}}\right] \\
& +\frac{1}{n} \mathrm{E}\left[\left\langle\mathbf{S}_{1}, \hat{\mathbf{S}}_{1}^{G}-\hat{\mathbf{S}}_{1}\right\rangle \mid \mathcal{E}_{\mathbf{S}}^{c} \cap \mathcal{E}_{\hat{\mathbf{U}}}\right] \operatorname{Pr}\left[\mathcal{E}_{\mathbf{S}}^{c} \cap \mathcal{E}_{\hat{\mathbf{U}}}\right] \\
& +\frac{1}{n} \underbrace{\mathrm{E}\left[\left\langle\mathbf{S}_{1}, \hat{\mathbf{S}}_{1}^{G}-\hat{\mathbf{S}}_{1}\right\rangle \mid \mathcal{E}_{\mathbf{S}}^{c} \cap \mathcal{E}_{\hat{\mathbf{U}}}^{c}\right]}_{=0} \operatorname{Pr}\left[\mathcal{E}_{\mathbf{S}}^{c} \cap \mathcal{E}_{\hat{\mathbf{U}}}^{c}\right] \\
& \stackrel{(a)}{\leq} \frac{1}{n} \mathrm{E}\left[\left\|\mathbf{S}_{1}\right\|^{2}+\left\|\hat{\mathbf{S}}_{1}^{\mathrm{G}}-\hat{\mathbf{S}}_{1}\right\|^{2} \mid \mathcal{E}_{\mathbf{S}}\right] \operatorname{Pr}\left[\mathcal{E}_{\mathbf{S}}\right] \\
& +\frac{1}{n} \mathrm{E}\left[\left\|\mathbf{S}_{1}\right\|\left\|\hat{\mathbf{S}}_{1}^{\mathrm{G}}-\hat{\mathbf{S}}_{1}\right\| \mid \mathcal{E}_{\mathbf{S}}^{c} \cap \mathcal{E}_{\hat{\mathbf{U}}}\right] \operatorname{Pr}\left[\mathcal{E}_{\hat{\mathbf{U}}}\right] \\
& \stackrel{(b)}{\leq} \frac{1}{n} \mathrm{E}\left[\left\|\mathbf{S}_{1}\right\|^{2} \mid \mathcal{E}_{\mathbf{S}}\right] \operatorname{Pr}\left[\mathcal{E}_{\mathbf{S}}\right]+36 \sigma^{2} \operatorname{Pr}\left[\mathcal{E}_{\mathbf{S}}\right] \\
& +\sqrt{\sigma^{2}(1+\epsilon)} \sqrt{36 \sigma^{2}} \operatorname{Pr}\left[\mathcal{E}_{\hat{\mathbf{U}}}\right] \\
& \stackrel{(c)}{\leq} \sigma^{2}\left(\epsilon+\operatorname{Pr}\left[\mathcal{E}_{\mathbf{S}}\right]\right)+36 \sigma^{2} \operatorname{Pr}\left[\mathcal{E}_{\mathbf{S}}\right] \\
& +6 \sigma^{2} \sqrt{1+\epsilon} \operatorname{Pr}\left[\mathcal{E}_{\hat{\mathbf{U}}}\right] \\
& =\sigma^{2}\left(\epsilon+37 \operatorname{Pr}\left[\mathcal{E}_{\mathbf{S}}\right]\right)+6 \sqrt{1+\epsilon} \operatorname{Pr}\left[\mathcal{E}_{\hat{\mathbf{U}}}\right] .
\end{aligned}
$$

In the first equality the third expectation term equals zero because by $\mathcal{E}_{\hat{\mathbf{U}}}^{c}$ we have $\left\|\hat{\mathbf{s}}_{1}^{\mathrm{G}}-\mathbf{s}_{1}\right\|=0$ and by $\mathcal{E}_{\mathbf{s}}^{c}$ the norm $\left\|\mathbf{s}_{1}\right\|$ is bounded. In (a) we have used two inequalities: in the first term the inner product is upper bounded by using (234). The second term is upper bounded by the Cauchy-Schwarz inequality and by $\operatorname{Pr}\left[\mathcal{E}_{\mathbf{S}}^{c} \cap \mathcal{E}_{\hat{\mathbf{U}}}\right] \leq \operatorname{Pr}\left[\mathcal{E}_{\hat{\mathbf{U}}}\right]$. In (b) we have used Lemma 12 and in (c) we have used Lemma 10.

\section{Lemma 14}

$$
\frac{1}{n} \mathrm{E}\left[\left\|\hat{\mathbf{S}}_{1}\right\|^{2}-\left\|\hat{\mathbf{S}}_{1}^{\mathrm{G}}\right\|^{2}\right] \leq 18 \sigma^{2} \operatorname{Pr}\left[\mathcal{E}_{\hat{\mathbf{U}}}\right]
$$

Proof:

$$
\begin{aligned}
\frac{1}{n} \mathrm{E}\left[\left\|\hat{\mathbf{S}}_{1}\right\|^{2}-\left\|\hat{\mathbf{S}}_{1}^{\mathrm{G}}\right\|^{2}\right]= & \frac{1}{n} \mathrm{E}\left[\left\|\hat{\mathbf{S}}_{1}\right\|^{2}-\left\|\hat{\mathbf{S}}_{1}^{\mathrm{G}}\right\|^{2} \mid \mathcal{E}_{\hat{\mathbf{U}}}\right] \operatorname{Pr}\left[\mathcal{E}_{\hat{\mathbf{U}}}\right] \\
& +\frac{1}{n} \mathrm{E}\left[\left\|\hat{\mathbf{S}}_{1}\right\|^{2}-\left\|\hat{\mathbf{S}}_{1}^{\mathrm{G}}\right\|^{2} \mid \mathcal{E}_{\hat{\mathbf{U}}}^{c}\right] \operatorname{Pr}\left[\mathcal{E}_{\hat{\mathbf{U}}}^{c}\right] \\
& \stackrel{(a)}{\leq} \frac{1}{n} \mathrm{E}\left[\left\|\hat{\mathbf{S}}_{1}\right\|^{2}+\left\|\hat{\mathbf{S}}_{1}^{\mathrm{G}}\right\|^{2} \mid \mathcal{E}_{\hat{\mathbf{U}}}\right] \operatorname{Pr}\left[\mathcal{E}_{\hat{\mathbf{U}}}\right]
\end{aligned}
$$




$$
\stackrel{(b)}{\leq} 18 \sigma^{2} \operatorname{Pr}\left[\mathcal{E}_{\hat{\mathbf{U}}}\right]
$$

where (a) follows since conditioned on $\mathcal{E}_{\hat{\mathbf{U}}}^{c}$ we have $\hat{\mathbf{s}}_{1}=\hat{\mathbf{s}}_{1}^{\mathrm{G}}$ and therefore $\left\|\hat{\mathbf{s}}_{1}\right\|^{2}-\left\|\hat{\mathbf{s}}_{1}^{\mathrm{G}}\right\|=0$, and where (b) follows by Lemma 12

Proof of Proposition 6 We show that the asymptotic normalized distortion resulting from the proposed vector-quantizer scheme, is the same as the asymptotic normalized distortion resulting from the genie-aided version of this scheme.

$$
\begin{aligned}
\frac{1}{n} \mathrm{E}[ & \left.\left\|\mathbf{S}_{1}-\hat{\mathbf{S}}_{1}\right\|^{2}\right]-\frac{1}{n} \mathrm{E}\left[\left\|\mathbf{S}_{1}-\hat{\mathbf{S}}_{1}^{\mathrm{G}}\right\|^{2}\right] \\
= & \frac{1}{n}\left(\mathrm{E}\left[\left\|\mathbf{S}_{1}\right\|^{2}\right]-2 \mathrm{E}\left[\left\langle\mathbf{S}_{1}, \hat{\mathbf{S}}_{1}\right\rangle\right]+\mathrm{E}\left[\left\|\hat{\mathbf{S}}_{1}\right\|^{2}\right]\right. \\
& \left.\quad-\mathrm{E}\left[\left\|\mathbf{S}_{1}\right\|^{2}\right]+2 \mathrm{E}\left[\left\langle\mathbf{S}_{1}, \hat{\mathbf{S}}_{1}^{\mathrm{G}}\right\rangle\right]-\mathrm{E}\left[\left\|\hat{\mathbf{S}}_{1}^{\mathrm{G}}\right\|^{2}\right]\right) \\
= & 2 \frac{1}{n} \mathrm{E}\left[\left\langle\mathbf{S}_{1}, \hat{\mathbf{S}}_{1}^{\mathrm{G}}-\hat{\mathbf{S}}_{1}\right\rangle\right]+\frac{1}{n} \mathrm{E}\left[\left\|\hat{\mathbf{S}}_{1}\right\|^{2}-\left\|\hat{\mathbf{S}}_{1}^{\mathrm{G}}\right\|^{2}\right] \\
& \stackrel{(a)}{\leq} 2 \sigma^{2}\left(\epsilon+37 \operatorname{Pr}\left[\mathcal{E}_{\mathbf{S}}\right]+6 \sqrt{1+\epsilon} \operatorname{Pr}\left[\mathcal{E}_{\hat{\mathbf{U}}}\right]\right)+18 \sigma^{2} \operatorname{Pr}\left[\mathcal{E}_{\hat{\mathbf{U}}}\right] \\
= & 2 \sigma^{2}\left(\epsilon+37 \operatorname{Pr}\left[\mathcal{E}_{\mathbf{S}}\right]+(6 \sqrt{1+\epsilon}+9) \operatorname{Pr}\left[\mathcal{E}_{\hat{\mathbf{U}}}\right]\right),
\end{aligned}
$$

where in step (a) we have used Lemma 13 and Lemma 14. Combining (A) with Lemma 5 and Lemma 6. gives that for every $\delta>0$ and $0.3>\epsilon>0$, there exists an $n^{\prime}(\delta, \epsilon)>0$ such that for all $\left(R_{1}, R_{2}, R_{\mathrm{c}}\right) \in \mathcal{R}(\epsilon)$ and $n>n^{\prime}(\delta, \epsilon)$

$$
\frac{1}{n} \mathrm{E}\left[\left\|\mathbf{S}_{1}-\hat{\mathbf{S}}_{1}\right\|^{2}\right]-\frac{1}{n} \mathrm{E}\left[\left\|\mathbf{S}_{1}-\hat{\mathbf{S}}_{1}^{\mathrm{G}}\right\|^{2}\right]<2 \sigma^{2}(\epsilon+(126 \sqrt{1+\epsilon}+226) \delta) .
$$

\section{A. Proof of rate constraint (102)}

Define

$$
\mathbf{w}\left(\mathbf{s}_{1}, \mathbf{s}_{2}, \mathcal{C}_{1}, \mathcal{C}_{2}, \mathcal{C}_{c}, \mathbf{z}\right)=\varsigma_{1}\left(\mathbf{y}-\left(\alpha \mathbf{v}^{*}+a_{2,1} \mathbf{u}_{2}^{*}\right)\right)+\varsigma_{2} a_{2,1} \mathbf{u}_{2}^{*}+\varsigma_{3} \alpha \mathbf{v}^{*}
$$

where

$$
\begin{aligned}
& \varsigma_{1}=\frac{\sigma^{2} a_{1,1}^{2}\left(1-2^{-2 R_{1}}\right)\left(1-\rho^{2}\left(1-2^{-2 R_{2}}\right)\left(1-2^{-2\left(R_{1}+R_{\mathrm{c}}\right)}\right)\right)}{\sigma^{2} a_{1,1}^{2}\left(1-2^{-2 R_{1}}\right)\left(1-\rho^{2}\left(1-2^{-2 R_{2}}\right)\left(1-2^{-2\left(R_{1}+R_{\mathrm{c}}\right)}\right)\right)+N\left(1-\bar{\rho}^{2}\right)} \\
& \varsigma_{2}=\frac{a_{1,1} \rho\left(1-2^{-2 R_{1}}\right) N}{a_{2,1}\left(\sigma^{2} a_{1,1}^{2}\left(1-2^{-2 R_{1}}\right)\left(1-\rho^{2}\left(1-2^{-2 R_{2}}\right)\left(1-2^{-2\left(R_{1}+R_{\mathrm{c}}\right)}\right)\right)+N\left(1-\bar{\rho}^{2}\right)\right)} \\
& \varsigma_{3}=\frac{-a_{1,1} \tilde{\rho}^{2} N}{\alpha\left(\sigma^{2} a_{1,1}^{2}\left(1-2^{-2 R_{1}}\right)\left(1-\rho^{2}\left(1-2^{-2 R_{2}}\right)\left(1-2^{-2\left(R_{1}+R_{\mathrm{c}}\right)}\right)\right)+N\left(1-\bar{\rho}^{2}\right)\right)} .
\end{aligned}
$$

In the remainder we shall use the shorthand notation $\mathbf{w}$ instead of $\mathbf{w}\left(\mathbf{s}_{1}, \mathbf{s}_{2}, \mathcal{C}_{1}, \mathcal{C}_{2}, \mathcal{C}_{c}, \mathbf{z}\right)$. We now start with a lemma that will be used to prove (102).

Lemma 15 Let $\varphi_{j} \in[0, \pi]$ be the angle between $\mathbf{w}$ and $\mathbf{u}_{1}(j)$, and let the set $\mathcal{E}_{\hat{\mathbf{U}}_{1}}^{\prime}$ be defined as

$$
\begin{aligned}
\mathcal{E}_{\hat{\mathbf{U}}_{1}}^{\prime} \triangleq\left\{\left(\mathbf{s}_{1}, \mathbf{s}_{2}, \mathcal{C}_{1}, \mathcal{C}_{2}, \mathcal{C}_{c}, \mathbf{z}\right): \exists \mathbf{u}_{1}(j) \in \mathcal{C}_{1} \backslash\left\{\mathbf{u}_{1}^{*}\right\}\right. \text { s.t. } \\
\left.\quad \cos \left(\varphi_{j}\right) \geq \sqrt{\frac{\bar{\beta}_{1} P_{1}\left(1-\tilde{\rho}^{2}\right)+N \tilde{\rho}^{2}-\bar{\rho}^{2} \bar{\beta}_{1} P_{1}}{\bar{\beta}_{1} P_{1}\left(1-\tilde{\rho}^{2}\right)+N-\bar{\rho}^{2}\left(\bar{\beta}_{1} P_{1}+N\right)}-\kappa^{\prime \prime} \epsilon}\right\},
\end{aligned}
$$


where $\kappa^{\prime \prime}$ is a positive constant determined by $P_{1}, P_{2}, N, \varsigma_{1}, \varsigma_{2}$ and $\varsigma_{3}$. Then,

$$
\mathcal{E}_{\hat{\mathbf{U}}_{1}} \cap \mathcal{E}_{\mathbf{S}}^{c} \cap \mathcal{E}_{\mathbf{X}}^{c} \cap \mathcal{E}_{\mathbf{Z}}^{c} \subseteq \mathcal{E}_{\hat{\mathbf{U}}_{1}}^{\prime} \cap \mathcal{E}_{\mathbf{S}}^{c} \cap \mathcal{E}_{\mathbf{X}}^{c} \cap \mathcal{E}_{\mathbf{Z}}^{c}
$$

and, in particular

$$
\operatorname{Pr}\left[\mathcal{E}_{\hat{\mathbf{U}}_{1}} \cap \mathcal{E}_{\mathbf{S}}^{c} \cap \mathcal{E}_{\mathbf{X}}^{c} \cap \mathcal{E}_{\mathbf{Z}}^{c}\right] \leq \operatorname{Pr}\left[\mathcal{E}_{\hat{\mathbf{U}}_{1}}^{\prime} \cap \mathcal{E}_{\mathbf{S}}^{c} \cap \mathcal{E}_{\mathbf{X}}^{c} \cap \mathcal{E}_{\mathbf{Z}}^{c}\right] .
$$

Proof: We first recall that for the event $\mathcal{E}_{\hat{\mathbf{U}}_{1}}$ to occur, there must exist a codeword $\mathbf{u}_{1}(j) \in$ $\mathcal{C}_{1} \backslash\left\{\mathbf{u}_{1}^{*}\right\}$ that satisfies the following three conditions

$$
\begin{aligned}
\left|\tilde{\rho}-\cos \varangle\left(\mathbf{u}_{1}(j), \mathbf{u}_{2}^{*}\right)\right| & \leq 7 \epsilon \\
\left|\cos \varangle\left(\mathbf{v}^{*}, \mathbf{u}_{1}(j)\right)\right| & \leq 3 \epsilon \\
\left\|\mathbf{y}-\mathbf{X}_{\mathbf{u}_{1}(j), \mathbf{v}^{*}, \mathbf{u}_{2}^{*}}\right\|^{2} & \leq\left\|\mathbf{y}-\mathbf{X}_{\mathbf{u}_{1}^{*}, \mathbf{v}^{*}, \mathbf{u}_{2}^{*}}\right\|^{2} .
\end{aligned}
$$

The proof is now based on a sequence of statements related to these three conditions:

A) For every $\left(\mathbf{s}_{1}, \mathbf{s}_{2}, \mathcal{C}_{1}, \mathcal{C}_{2}, \mathcal{C}_{c}, \mathbf{z}\right) \in \mathcal{E}_{\mathbf{X}}^{c}$ and every $\mathbf{u} \in \mathcal{S}_{1}$, where $\mathcal{S}_{1}$ is the surface area of the codeword sphere of $\mathcal{C}_{1}$ defined in the code construction,

$$
\begin{aligned}
& \left|\tilde{\rho}-\cos \varangle\left(\mathbf{u}, \mathbf{u}_{2}^{*}\right)\right| \leq 7 \epsilon \\
& \quad \Longrightarrow\left|n \tilde{\rho} \sqrt{\bar{\beta}_{1} \bar{\beta}_{2} P_{1} P_{2}}-\left\langle a_{1,1} \mathbf{u}, a_{2,1} \mathbf{u}_{2}^{*}\right\rangle\right| \leq 7 n \sqrt{\bar{\beta}_{1} \bar{\beta}_{2} P_{1} P_{2}} \epsilon .
\end{aligned}
$$

Statement A) follows by rewriting $\cos \varangle\left(\mathbf{u}, \mathbf{u}_{2}^{*}\right)$ as $\left\langle\mathbf{u}, \mathbf{u}_{2}^{*}\right\rangle /\left(\|\mathbf{u}\|\left\|\mathbf{u}_{2}^{*}\right\|\right)$, and then multiplying the inequality on the 1.h.s. of (117) by $\left\|a_{1,1} \mathbf{u}\right\| \cdot\left\|a_{2,1} \mathbf{u}_{2}^{*}\right\|$ and recalling that $\left\|a_{1,1} \mathbf{u}\right\|=\sqrt{n \bar{\beta}_{1} P_{1}}$ and that $\left\|a_{2,1} \mathbf{u}_{2}^{*}\right\|=\sqrt{n \bar{\beta}_{2} P_{2}}$.

A1) For every $\left(\mathbf{s}_{1}, \mathbf{s}_{2}, \mathcal{C}_{1}, \mathcal{C}_{2}, \mathcal{C}_{c}, \mathbf{z}\right) \in \mathcal{E}_{\mathbf{X}}^{c}$

$$
\begin{aligned}
& \left|\cos \varangle\left(\mathbf{v}^{*}, \mathbf{u}\right)\right| \leq 3 \epsilon \\
& \Longrightarrow \quad\left|\left\langle\alpha \mathbf{v}^{*}, a_{1,1} \mathbf{u}\right\rangle\right| \leq 3\left\|\alpha \mathbf{v}^{*}\right\| \sqrt{n \overline{\beta_{1} P_{1}} \epsilon .}
\end{aligned}
$$

Statement A1) follows by rewriting $\cos \varangle\left(\mathbf{v}^{*}, \mathbf{u}\right)$ as $\left\langle\mathbf{v}^{*}, \mathbf{u}\right\rangle /\left(\left\|\mathbf{v}^{*}\right\|\|\mathbf{u}\|\right)$, and then multiplying the inequality on the 1.h.s. of (118) by $\left\|\alpha \mathbf{v}^{*}\right\| \cdot\left\|a_{1,1} \mathbf{u}\right\|$.

A2) For every $\left(\mathbf{s}_{1}, \mathbf{s}_{2}, \mathcal{C}_{1}, \mathcal{C}_{2}, \mathcal{C}_{c}, \mathbf{z}\right) \in \mathcal{E}_{\mathbf{X}}^{c}$

$$
\begin{aligned}
& \left|\bar{\rho}-\cos \varangle\left(\mathbf{v}^{*}, \mathbf{u}_{2}^{*}\right)\right| \leq 7 \epsilon \\
& \quad \Longrightarrow\left|\left\|\alpha \mathbf{v}^{*}\right\| \sqrt{n \bar{\beta}_{2} P_{2}} \bar{\rho}-\left\langle\alpha \mathbf{v}^{*}, a_{2,1} \mathbf{u}_{2}^{*}\right\rangle\right| \leq 7 \epsilon\left\|\alpha \mathbf{v}^{*}\right\| \sqrt{n \bar{\beta}_{2} P_{2}} .
\end{aligned}
$$

Statement A2) follows by rewriting $\cos \varangle\left(\mathbf{v}^{*}, \mathbf{u}_{2}^{*}\right)$ as $\left\langle\mathbf{v}^{*}, \mathbf{u}_{2}^{*}\right\rangle /\left(\left\|\mathbf{v}^{*}\right\|\left\|\mathbf{u}_{2}^{*}\right\|\right)$, and then multiplying the inequality on the 1.h.s. of (119) by $\left\|\alpha \mathbf{v}^{*}\right\| \cdot\left\|a_{2,1} \mathbf{u}_{2}^{*}\right\|$.

B) For every $\left(\mathbf{s}_{1}, \mathbf{s}_{2}, \mathcal{C}_{1}, \mathcal{C}_{2}, \mathcal{C}_{c}, \mathbf{z}\right) \in \mathcal{E}_{\mathbf{X}}^{c} \cap \mathcal{E}_{\mathbf{Z}}^{c}$ and every $\mathbf{u} \in \mathcal{S}_{1}$

$$
\begin{aligned}
& \mid \mathbf{y}-\mathbf{X}_{\mathbf{u}, \mathbf{v}^{*}, \mathbf{u}_{2}^{*}}\left\|^{2} \leq\right\| \mathbf{y}-\mathbf{X}_{\mathbf{u}_{1}^{*}, \mathbf{v}^{*}, \mathbf{u}_{2}^{*}} \|^{2} \\
& \quad \Longrightarrow\left\langle\mathbf{y}-\left(a_{2,1} \mathbf{u}_{2}^{*}+\alpha \mathbf{v}^{*}\right), a_{1,1} \mathbf{u}\right\rangle \geq n\left(\bar{\beta}_{1} P_{1}-\sqrt{\bar{\beta}_{1} P_{1} N \epsilon}\right) .
\end{aligned}
$$

Statement B) follows from rewriting the inequality on the 1.h.s. of (120) as

$$
\left\|\mathbf{y}-\left(a_{2,1} \mathbf{u}_{2}^{*}+\alpha \mathbf{v}^{*}\right)-a_{1,1} \mathbf{u}\right\|^{2} \leq\left\|\mathbf{y}-\left(a_{2,1} \mathbf{u}_{2}^{*}+\alpha \mathbf{v}^{*}\right)-a_{1,1} \mathbf{u}_{1}^{*}\right\|^{2}
$$


or equivalently as

$$
\begin{aligned}
\left\langle\mathbf{y}-\left(a_{2,1} \mathbf{u}_{2}^{*}+\alpha \mathbf{v}^{*}\right), a_{1,1} \mathbf{u}\right\rangle & \geq\left\langle\mathbf{y}-\left(a_{2,1} \mathbf{u}_{2}^{*}+\alpha \mathbf{v}^{*}\right), a_{1,1} \mathbf{u}_{1}^{*}\right\rangle \\
& =\left\langle a_{1,1} \mathbf{u}_{1}^{*}+\mathbf{z}, a_{1,1} \mathbf{u}_{1}^{*}\right\rangle \\
& =\left\|a_{1,1} \mathbf{u}_{1}^{*}\right\|^{2}+\left\langle\mathbf{z}, a_{1,1} \mathbf{u}_{1}^{*}\right\rangle .
\end{aligned}
$$

It now follows from the equivalence of the first inequality in (120) with 121) that for $\left(\mathbf{s}_{1}, \mathbf{s}_{2}, \mathcal{C}_{1}, \mathcal{C}_{2}, \mathcal{C}_{c}, \mathbf{z}\right) \in \mathcal{E}_{\mathbf{Z}}^{c}$, the first inequality in 120 ) can only hold if

$$
\left\langle\mathbf{y}-\left(a_{2,1} \mathbf{u}_{2}^{*}+\alpha \mathbf{v}^{*}\right), a_{1,1} \mathbf{u}\right\rangle \geq n\left(\bar{\beta}_{1} P_{1}-\sqrt{\bar{\beta}_{1} P_{1} N \epsilon}\right),
$$

thus establising B).

C) For every $\left(\mathbf{s}_{1}, \mathbf{s}_{2}, \mathcal{C}_{1}, \mathcal{C}_{2}, \mathcal{C}_{c}, \mathbf{z}\right) \in \mathcal{E}_{\mathbf{X}}^{c} \cap \mathcal{E}_{\mathbf{Z}}^{c}$ and every $\mathbf{u} \in \mathcal{S}_{1}$,

$$
\begin{gathered}
\left|\tilde{\rho}-\cos \varangle\left(\mathbf{u}, \mathbf{u}_{2}^{*}\right)\right| \leq 7 \epsilon \quad \text { and } \quad \mid \mathbf{y}-\mathbf{X}_{\mathbf{u}, \mathbf{v}^{*}, \mathbf{u}_{2}^{*}}\left\|^{2} \leq\right\| \mathbf{y}-\mathbf{X}_{\mathbf{u}_{1}^{*}, \mathbf{v}^{*}, \mathbf{u}_{2}^{*}} \|^{2} \\
\Longrightarrow \\
\left\|a_{1,1} \mathbf{u}-\mathbf{w}\right\|^{2} \leq n \bar{\beta}_{1} P_{1}-2\left(n \varsigma_{1}\left(\bar{\beta}_{1} P_{1}-\sqrt{\bar{\beta}_{1} P_{1} N}\right)+n \varsigma_{2}\left(\sqrt{\bar{\beta}_{1} \bar{\beta}_{2} P_{1} P_{2}}(\tilde{\rho}-7 \epsilon)\right)\right. \\
\left.\quad-\varsigma_{3}\left(\left\|\alpha \mathbf{v}^{*}\right\| \sqrt{n \bar{\beta}_{1} P_{1}} 3 \epsilon\right)\right)+\|\mathbf{w}\|^{2} .
\end{gathered}
$$

Statement C) is obtained as follows:

$$
\begin{aligned}
& \left\|a_{1,1} \mathbf{u}-\mathbf{w}\right\|^{2}=\left\|a_{1,1} \mathbf{u}\right\|^{2}-2\left\langle a_{1,1} \mathbf{u}, \mathbf{w}\right\rangle+\|\mathbf{w}\|^{2} \\
& =\left\|a_{1,1} \mathbf{u}\right\|^{2}-2\left\langle a_{1,1} \mathbf{u}, \varsigma_{1}\left(a_{1,1} \mathbf{u}_{1}^{*}+\mathbf{z}\right)+\varsigma_{2} a_{2,1} \mathbf{u}_{2}^{*}+\varsigma_{3} \alpha \mathbf{v}^{*}\right\rangle+\|\mathbf{w}\|^{2} \\
& =n \bar{\beta}_{1} P_{1}-2\left[\varsigma_{1}\left\langle a_{1,1} \mathbf{u}, a_{1,1} \mathbf{u}_{1}^{*}+\mathbf{z}\right\rangle+\varsigma_{2}\left\langle a_{1,1} \mathbf{u}, a_{2,1} \mathbf{u}_{2}^{*}\right\rangle+\varsigma_{3}\left\langle a_{1,1} \mathbf{u}, \alpha \mathbf{v}^{*}\right\rangle\right]+\|\mathbf{w}\|^{2} \\
& \quad \stackrel{(a)}{\leq} n \bar{\beta}_{1} P_{1}-2\left(n \varsigma_{1}\left(\bar{\beta}_{1} P_{1}-\sqrt{\bar{\beta}_{1} P_{1} N \epsilon}\right)+n \varsigma_{2}\left(\sqrt{\bar{\beta}_{1} \bar{\beta}_{2} P_{1} P_{2}}(\tilde{\rho}-7 \epsilon)\right)\right. \\
& \left.\quad-\varsigma_{3}\left(\left\|\alpha \mathbf{v}^{*}\right\| \sqrt{n \bar{\beta}_{1} P_{1}} 3 \epsilon\right)\right)+\|\mathbf{w}\|^{2},
\end{aligned}
$$

where in (a) we have used Statement A), Statement A1) and Statement B).

D) For every $\left(\mathbf{s}_{1}, \mathbf{s}_{2}, \mathcal{C}_{1}, \mathcal{C}_{2}, \mathcal{C}_{c}, \mathbf{z}\right) \in \mathcal{E}_{\mathbf{X}}^{c} \cap \mathcal{E}_{\mathbf{Z}}^{c}$

$$
\begin{aligned}
\|\mathbf{w}\|^{2} \leq & n\left(\varsigma_{1}{ }^{2}\left(\bar{\beta}_{1} P_{1}+N\right)+2 \varsigma_{1} \varsigma_{2} \sqrt{\bar{\beta}_{1} \bar{\beta}_{2} P_{1} P_{2}} \tilde{\rho}+\varsigma_{2}{ }^{2} \bar{\beta}_{2} P_{2}+2 \varsigma_{2} \varsigma_{3}\|\alpha \mathbf{v}\| \sqrt{n \bar{\beta}_{2} P_{2}} \bar{\rho}\right. \\
& \left.+\frac{1}{n} \varsigma_{3}{ }^{2} \alpha^{2}\left\|\mathbf{v}^{*}\right\|^{2}+\kappa \epsilon\right),
\end{aligned}
$$

where $\kappa$ depends on $P_{1}, P_{2}, N, \varsigma_{1}, \varsigma_{2}$ and $\varsigma_{3}$ only.

Statement D) is obtained as follows:

$$
\begin{aligned}
& \|\mathbf{w}\|^{2}=\left\|\varsigma_{1}\left(a_{1,1} \mathbf{u}_{1}^{*}+\mathbf{z}\right)+\varsigma_{2} a_{2,1} \mathbf{u}_{2}^{*}+\varsigma_{3} \alpha \mathbf{v}^{*}\right\|^{2} \\
& =\varsigma_{1}^{2}\left\|a_{1,1} \mathbf{u}_{1}^{*}+\mathbf{z}\right\|^{2}+2 \varsigma_{1} \varsigma_{2}\left\langle a_{1,1} \mathbf{u}_{1}^{*}+\mathbf{z}, a_{2,1} \mathbf{u}_{2}^{*}\right\rangle+\varsigma_{2}{ }^{2}\left\|a_{2,1} \mathbf{u}_{2}^{*}\right\|^{2} \\
& \quad+2 \varsigma_{1} \varsigma_{3}\left\langle a_{1,1} \mathbf{u}_{1}^{*}+\mathbf{z}, \alpha \mathbf{v}^{*}\right\rangle+2 \varsigma_{2} \varsigma_{3}\left\langle a_{2,1} \mathbf{u}_{2}^{*}, \alpha \mathbf{v}^{*}\right\rangle+\varsigma_{3}{ }^{2}\left\|\alpha \mathbf{v}^{*}\right\|^{2} \\
& =\varsigma_{1}^{2}\left(\left\|a_{1,1} \mathbf{u}_{1}^{*}\right\|^{2}+2\left\langle a_{1,1} \mathbf{u}_{1}^{*}, \mathbf{z}\right\rangle+\|\mathbf{z}\|^{2}\right)+2 \varsigma_{1} \varsigma_{2}\left(\left\langle a_{1,1} \mathbf{u}_{1}^{*}, a_{2,1} \mathbf{u}_{2}^{*}\right\rangle+\left\langle\mathbf{z}, a_{2,1} \mathbf{u}_{2}^{*}\right\rangle\right) \\
& \quad+\varsigma_{2}{ }^{2}\left(n \bar{\beta}_{2} P_{2}\right)+2 \varsigma_{1} \varsigma_{3}\left\langle\mathbf{z}, \alpha \mathbf{v}^{*}\right\rangle+2 \varsigma_{2} \varsigma_{3}\left\langle a_{2,1} \mathbf{u}_{2}^{*}, \alpha \mathbf{v}^{*}\right\rangle+\varsigma_{3}{ }^{2} \alpha^{2}\left\|\mathbf{v}^{*}\right\|^{2} \\
& \quad \stackrel{(a)}{\leq} n\left(\varsigma_{1}^{2}\left(\bar{\beta}_{1} P_{1}+N\right)+2 \varsigma_{1} \varsigma_{2} \sqrt{\bar{\beta}_{1} \bar{\beta}_{2} P_{1} P_{2} \tilde{\rho}+\varsigma_{2}{ }^{2}\left(\bar{\beta}_{2} P_{2}\right)+2 \varsigma_{2} \varsigma_{3}\|\alpha \mathbf{v}\| \sqrt{n \bar{\beta}_{2} P_{2} \bar{\rho}}}\right.
\end{aligned}
$$




$$
\left.+\frac{1}{n} \varsigma_{3}^{2} \alpha^{2}\left\|\mathbf{v}^{*}\right\|^{2}+\kappa \epsilon\right)
$$

where in (a) we have used that $\left(\mathbf{s}_{1}, \mathbf{s}_{2}, \mathcal{C}_{1}, \mathcal{C}_{2}, \mathcal{C}_{c}, \mathbf{z}\right) \in \mathcal{E}_{\mathbf{X}}^{c}$, and statements A) and A2).

E) For every $\left(\mathbf{s}_{1}, \mathbf{s}_{2}, \mathcal{C}_{1}, \mathcal{C}_{2}, \mathcal{C}_{c}, \mathbf{z}\right) \in \mathcal{E}_{\mathbf{X}}^{c} \cap \mathcal{E}_{\mathbf{Z}}^{c}$ and an arbitrary $\mathbf{u} \in \mathcal{S}_{1}$,

$$
\begin{aligned}
& \left|\tilde{\rho}-\cos \varangle\left(\mathbf{u}, \mathbf{u}_{2}^{*}\right)\right| \leq 7 \epsilon \text { and }\left|\bar{\rho}-\cos \varangle\left(\mathbf{v}^{*}, \mathbf{u}_{2}^{*}\right)\right| \leq 7 \epsilon \text { and } \\
& \left\|\mathbf{y}-\left(a_{1,1} \mathbf{u}+a_{2,1} \mathbf{u}_{2}^{*}+\left(a_{1,2}+a_{2,2}\right) \mathbf{v}^{*}\right)\right\|^{2} \leq\left\|\mathbf{y}-\left(a_{1,1} \mathbf{u}_{1}^{*}+a_{2,1} \mathbf{u}_{2}^{*}+\alpha \mathbf{v}^{*}\right)\right\|^{2} \\
& \Longrightarrow\left\|a_{1,1} \mathbf{u}-\mathbf{w}\right\|^{2} \leq \Upsilon(\epsilon),
\end{aligned}
$$

where

$$
\Upsilon(\epsilon)=n \frac{\bar{\beta}_{1} P_{1} N\left(1-\tilde{\rho}^{2}-\bar{\rho}^{2}\right)}{\bar{\beta}_{1} P_{1}\left(1-\tilde{\rho}^{2}\right)+N-\bar{\rho}^{2}\left(\bar{\beta}_{1} P_{1}+N\right)}+n \kappa^{\prime} \epsilon,
$$

and where $\kappa^{\prime}$ only depends on $P_{1}, N_{1}, N_{2}, \varsigma_{1}, \varsigma_{2}$ and $\varsigma_{3}$.

Statement E) follows from combining Statement C) with Statement D) and the explicit values of $\varsigma_{1}, \varsigma_{2}$ and $\varsigma_{3}$ given in (112).

F) For every $\mathbf{u} \in \mathcal{S}_{1}$, denote by $\varphi \in[0, \pi]$ the angle between $\mathbf{u}$ and $\mathbf{w}$, and let

$$
\begin{aligned}
\mathcal{B}\left(\mathbf{s}_{1}, \mathbf{s}_{2}, \mathbf{u}_{1}^{*}, \mathbf{v}^{*}, \mathbf{u}_{2}^{*}, \mathbf{z}\right) \triangleq\left\{\mathbf{u} \in S_{1}^{(n)}\right. & : \cos (\varphi) \geq \sqrt{1-\frac{\Upsilon(\epsilon)}{n \bar{\beta}_{1} P_{1}}} \\
& \left.=\sqrt{\frac{\bar{\beta}_{1} P_{1}\left(1-\tilde{\rho}^{2}\right)+N \tilde{\rho}^{2}-\bar{\rho}^{2} \bar{\beta}_{1} P_{1}}{\bar{\beta}_{1} P_{1}\left(1-\tilde{\rho}^{2}\right)+N-\bar{\rho}^{2}\left(\bar{\beta}_{1} P_{1}+N\right)}-\kappa^{\prime \prime} \epsilon}\right\},
\end{aligned}
$$

where $\kappa^{\prime \prime}$ only depends on $P_{1}, N_{1}, N_{2}, \varsigma_{1}, \varsigma_{2}$ and $\varsigma_{3}$, and where we assume $\epsilon$ sufficiently small such that

$$
\frac{\bar{\beta}_{1} P_{1}\left(1-\tilde{\rho}^{2}\right)+N \tilde{\rho}^{2}-\bar{\rho}^{2} \bar{\beta}_{1} P_{1}}{\bar{\beta}_{1} P_{1}\left(1-\tilde{\rho}^{2}\right)+N-\bar{\rho}^{2}\left(\bar{\beta}_{1} P_{1}+N\right)}-\kappa^{\prime \prime} \epsilon>0 .
$$

Then, for every $\left(\mathbf{s}_{1}, \mathbf{s}_{2}, \mathcal{C}_{1}, \mathcal{C}_{2}, \mathcal{C}_{c}, \mathbf{z}\right) \in \mathcal{E}_{\mathbf{X}}^{c} \cap \mathcal{E}_{\mathbf{Z}}^{c}$,

$$
\begin{aligned}
& \left|\tilde{\rho}-\cos \varangle\left(\mathbf{u}, \mathbf{u}_{2}^{*}\right)\right| \leq 7 \epsilon \quad \text { and } \quad\left|\bar{\rho}-\cos \varangle\left(\mathbf{v}^{*}, \mathbf{u}_{2}^{*}\right)\right| \leq 7 \epsilon \quad \text { and } \\
& \left\|\mathbf{y}-\left(a_{1,1} \mathbf{u}+a_{2,1} \mathbf{u}_{2}^{*}+\alpha \mathbf{v}^{*}\right)\right\|^{2} \leq\left\|\mathbf{y}-\left(a_{1,1} \mathbf{u}_{1}^{*}+a_{2,1} \mathbf{u}_{2}^{*}+\alpha \mathbf{v}^{*}\right)\right\|^{2} \\
& \Longrightarrow \mathbf{u} \in \mathcal{B}\left(\mathbf{s}_{1}, \mathbf{s}_{2}, \mathbf{u}_{1}^{*}, \mathbf{v}^{*}, \mathbf{u}_{2}^{*}, \mathbf{z}\right) .
\end{aligned}
$$

Statement F) follows from Statement E) by noting that if $\mathbf{w} \neq 0$ and $1-\frac{\Upsilon(\epsilon)}{n \bar{\beta}_{1} P_{1}}>0$, then

$$
\left.\begin{array}{l}
\left\|a_{1,1} \mathbf{u}\right\|^{2}=n \bar{\beta}_{1} P_{1} \\
\left\|a_{1,1} \mathbf{u}-\mathbf{w}\right\|^{2} \leq \Upsilon(\epsilon)
\end{array}\right\} \Longrightarrow \cos \varangle(\mathbf{u}, \mathbf{w}) \geq \sqrt{1-\frac{\Upsilon(\epsilon)}{n \bar{\beta}_{1} P_{1}}} .
$$

To see this, first note that every $a_{1,1} \mathbf{u}$, where $\mathbf{u} \in \mathcal{S}_{1}$, satisfying the condition on the 1.h.s. of (122) lies within a sphere of radius $\sqrt{\Upsilon(\epsilon)}$ centered at $\mathbf{w}$. In addition, for every $\mathbf{u} \in \mathcal{S}_{1}$ we have that $a_{1,1} \mathbf{u}$ also lies on the centered $\mathbb{R}^{n}$-sphere of radius $\sqrt{n \bar{\beta}_{1} P_{1}}$. Hence, every $\mathbf{u} \in \mathcal{S}_{1}$ satisfying the condition on the 1.h.s. of (122) lies in the intersection of these two regions, which is a polar cap on the centered sphere of radius $\sqrt{n \bar{\beta}_{1} P_{1}}$. The area of this polar cap is outer bounded as follows. Let $\mathbf{r}$ be an arbitrary point on the boundary of this polar cap. The half-angle of the polar cap would be maximized if $\mathbf{w}$ and $\mathbf{r}-\mathbf{w}$ would lie perpendicular to each other. Hence, every $\mathbf{u} \in \mathcal{S}_{1}^{(n)}$ satisfying the upper conditions of (122), also satisfies

$$
\cos \varphi \geq \sqrt{1-\frac{\Upsilon(\epsilon)}{n \bar{\beta}_{1} P_{1}}}
$$




$$
=\sqrt{\frac{\bar{\beta}_{1} P_{1}\left(1-\tilde{\rho}^{2}\right)+N \tilde{\rho}^{2}-\bar{\rho}^{2} \bar{\beta}_{1} P_{1}}{\bar{\beta}_{1} P_{1}\left(1-\tilde{\rho}^{2}\right)+N-\bar{\rho}^{2}\left(\bar{\beta}_{1} P_{1}+N\right)}-\kappa^{\prime \prime} \epsilon},
$$

where we assume $\epsilon$ sufficiently small such that $1-\frac{\Upsilon(\epsilon)}{n \beta_{1} P_{1}}>0$ and where $\kappa^{\prime \prime}=\frac{\kappa^{\prime}}{n \beta_{1} P_{1}}$.

The proof of Lemma 15 is now concluded by noticing that the set $\mathcal{E}_{\hat{\mathbf{U}}_{1}}^{\prime}$, defined in [113), is the set of tuples $\left(\mathbf{s}_{1}, \mathbf{s}_{2}, \mathcal{C}_{1}, \mathcal{C}_{2}, \mathcal{C}_{c}, \mathbf{z}\right)$ for which there exists a $\mathbf{u}_{1}(j) \in \mathcal{C}_{1} \backslash\left\{\mathbf{u}_{1}^{*}\right\}$ such that $\mathbf{u}_{1}(j) \in \mathcal{B}\left(\mathbf{s}_{1}, \mathbf{s}_{2}, \mathbf{u}_{1}^{*}, \mathbf{v}^{*}, \mathbf{u}_{2}^{*}, \mathbf{z}\right)$. Thus, by Statement F) and by the definition of $\mathcal{E}_{\hat{\mathbf{U}}_{1}}$ in 87] it follows that

$$
\mathcal{E}_{\hat{\mathbf{U}}_{1}} \cap \mathcal{E}_{\mathbf{S}}^{c} \cap \mathcal{E}_{\mathbf{X}}^{c} \cap \mathcal{E}_{\mathbf{Z}}^{c} \subseteq \mathcal{E}_{\hat{\mathbf{U}}_{1}}^{\prime} \cap \mathcal{E}_{\mathbf{S}}^{c} \cap \mathcal{E}_{\mathbf{X}}^{c} \cap \mathcal{E}_{\mathbf{Z}}^{c}
$$

and therefore

$$
\operatorname{Pr}\left[\mathcal{E}_{\hat{\mathbf{U}}_{1}} \cap \mathcal{E}_{\mathbf{S}}^{c} \cap \mathcal{E}_{\mathbf{X}}^{c} \cap \mathcal{E}_{\mathbf{Z}}^{c}\right] \leq \operatorname{Pr}\left[\mathcal{E}_{\hat{\mathbf{U}}_{1}}^{\prime} \cap \mathcal{E}_{\mathbf{S}}^{c} \cap \mathcal{E}_{\mathbf{X}}^{c} \cap \mathcal{E}_{\mathbf{Z}}^{c}\right]
$$

We now state one more lemma that will be used for the proof of (102).

Lemma 16 For every $\Delta \in(0,1]$, let the set $\mathcal{G}$ be given by

$$
\mathcal{G}=\left\{\left(\mathbf{s}_{1}, \mathbf{s}_{2}, \mathcal{C}_{1}, \mathcal{C}_{2}, \mathcal{C}_{c}, \mathbf{z}\right): \exists \mathbf{u}_{1}(j) \in \mathcal{C}_{1} \backslash\left\{\mathbf{u}_{1}^{*}\right\} \text { s.t. } \cos \varangle\left(\mathbf{w}, \mathbf{u}_{1}(j)\right) \geq \Delta\right\},
$$

where $\mathbf{w}$ is defined in (111). Then,

$$
R_{1}<-\frac{1}{2} \log \left(1-\Delta^{2}\right) \quad \Longrightarrow \quad\left(\lim _{n \rightarrow \infty} \operatorname{Pr}\left[\mathcal{G} \mid \mathcal{E}_{\mathbf{X}_{1}}^{c}\right]=0, \epsilon>0\right),
$$

where $\mathcal{E}_{\mathbf{X}_{1}}$ is defined in (94).

Proof: The proof follows from upper-bounding in every point on $\mathcal{S}_{1}$ the density of every $\mathbf{u}_{1}(j) \in \mathcal{C}_{1} \backslash\left\{\mathbf{u}_{1}^{*}\right\}$ and then using a standard argument from sphere-packing. See [1, Appendix D-E2].

Next,

$$
\begin{aligned}
\operatorname{Pr}\left[\mathcal{E}_{\hat{\mathbf{U}}_{1}} \cap \mathcal{E}_{\mathbf{S}}^{c} \cap \mathcal{E}_{\mathbf{X}}^{c} \cap \mathcal{E}_{\mathbf{Z}}^{c}\right] \stackrel{(a)}{\stackrel{(a)}{\leq}} \operatorname{Pr}\left[\mathcal{E}_{\hat{\mathbf{U}}_{1}}^{\prime} \cap \mathcal{E}_{\mathbf{S}}^{c} \cap \mathcal{E}_{\mathbf{X}}^{c} \cap \mathcal{E}_{\mathbf{Z}}^{c}\right] \\
\stackrel{(b)}{\leq} \operatorname{Pr}\left[\mathcal{E}_{\hat{\mathbf{U}}_{1}}^{\prime} \mid \mathcal{E}_{\mathbf{X}_{1}}^{c}\right]
\end{aligned}
$$

where (a) follows by Lemma 15 and (b) follows because $\mathcal{E}_{\mathbf{X}}^{c} \subseteq \mathcal{E}_{\mathbf{X}_{1}}^{c}$. The proof of 102) is now completed by combining (124) with Lemma 16. This gives that for every $\delta>0$ and every $\epsilon>0$ there exists some $n^{\prime}(\delta, \epsilon)$ such that for all $n>n^{\prime}(\delta, \epsilon)$, we have

$$
\operatorname{Pr}\left[\mathcal{E}_{\hat{\mathbf{U}}_{1}} \cap \mathcal{E}_{\mathbf{S}}^{c} \cap \mathcal{E}_{\mathbf{X}}^{c} \cap \mathcal{E}_{\mathbf{Z}}^{c}\right] \leq \operatorname{Pr}\left[\mathcal{E}_{\hat{\mathbf{U}}_{1}}^{\prime} \mid \mathcal{E}_{\mathbf{X}_{1}}^{c}\right]<\delta,
$$

whenever

$$
R_{1}<\frac{1}{2} \log \left(\frac{\bar{\beta}_{1} P_{1}\left(1-\tilde{\rho}^{2}-\bar{\rho}^{2}\right)+N\left(1-\bar{\rho}^{2}\right)}{N\left(1-\tilde{\rho}^{2}\right)-\bar{\rho}^{2} N}-\kappa_{1} \epsilon\right),
$$

where $\kappa_{1}$ is a positive constant determined by $P_{1}, P_{2}, N, \varsigma_{1}, \varsigma_{2}$ and $\varsigma_{3}$.

\section{B. Proof of rate constraint (103)}

Define

$$
\mathbf{w}\left(\mathbf{s}_{1}, \mathbf{s}_{2}, \mathcal{C}_{1}, \mathcal{C}_{2}, \mathcal{C}_{c}, \mathbf{z}\right)=\varsigma_{1}\left(\mathbf{y}-\left(a_{1,1} \mathbf{u}_{1}^{*}+\alpha \mathbf{v}^{*}\right)\right)+\varsigma_{2} a_{1,1} \mathbf{u}_{1}^{*}+\varsigma_{3} \alpha \mathbf{v}^{*}
$$


where

$$
\begin{aligned}
& \varsigma_{1}=\frac{\sigma^{2} a_{2,1}^{2}\left(1-2^{-2 R_{2}}\right)\left(1-\bar{\rho}^{2}-\tilde{\rho}^{2}\right)}{\sigma^{2} a_{2,1}^{2}\left(1-2^{-2 R_{2}}\right)\left(1-\bar{\rho}^{2}-\tilde{\rho}^{2}\right)+N} \\
& \varsigma_{2}=\frac{a_{2,1}\left(1-2^{-2 R_{2}}\right) \rho\left(1-\bar{\rho}^{2}\right) N}{a_{1,1}\left(\sigma^{2} a_{2,1}^{2}\left(1-2^{-2 R_{2}}\right)\left(1-\bar{\rho}^{2}-\tilde{\rho}^{2}\right)+N\right)} \\
& \varsigma_{3}=\frac{a_{2,1}\left(1-2^{-2 R_{2}}\right) \rho\left(1-\tilde{\rho}^{2}\right) N}{\alpha\left(\sigma^{2} a_{2,1}^{2}\left(1-2^{-2 R_{2}}\right)\left(1-\bar{\rho}^{2}-\tilde{\rho}^{2}\right)+N\right)} .
\end{aligned}
$$

We now start with a lemma that will be used to prove (103).

Lemma 17 Let $\varphi_{j} \in[0, \pi]$ be the angle between $\mathbf{w}$ and $\mathbf{u}_{2}(j)$, and let the set $\mathcal{E}_{\hat{\mathbf{U}}_{2}}^{\prime}$ be defined as

$$
\begin{aligned}
& \mathcal{E}_{\hat{\mathbf{U}}_{2}}^{\prime} \triangleq\left\{\left(\mathbf{s}_{1}, \mathbf{s}_{2}, \mathcal{C}_{1}, \mathcal{C}_{2}, \mathcal{C}_{c}, \mathbf{z}\right): \exists \mathbf{u}_{2}(j) \in \mathcal{C}_{2} \backslash\left\{\mathbf{u}_{2}^{*}\right\}\right. \text { s.t. } \\
& \left.\cos \left(\varphi_{j}\right) \geq \sqrt{\frac{\bar{\beta}_{2} P_{2}\left(1-\tilde{\rho}^{2}\right)+N \tilde{\rho}^{2}-\bar{\rho}^{2}\left(\bar{\beta}_{2} P_{2}-N\right)}{\bar{\beta}_{2} P_{2}\left[1-\tilde{\rho}^{2}\right]+N-\bar{\rho}^{2} \bar{\beta}_{2} P_{2}}-\frac{N^{2} \tilde{\rho}^{2} \bar{\rho}^{2}\left(2+\tilde{\rho}^{2}\right)}{\left(\bar{\beta}_{2} P_{2}\left[1-\bar{\rho}^{2}-\tilde{\rho}^{2}\right]+N\right)^{2}}-\kappa^{\prime \prime} \epsilon}\right\},
\end{aligned}
$$

where $\kappa^{\prime \prime}$ is a positive constant determined by $P_{1}, P_{2}, N, \varsigma_{1}, \varsigma_{2}$ and $\varsigma_{3}$. Then,

$$
\mathcal{E}_{\hat{\mathbf{U}}_{2}} \cap \mathcal{E}_{\mathbf{S}}^{c} \cap \mathcal{E}_{\mathbf{X}}^{c} \cap \mathcal{E}_{\mathbf{Z}}^{c} \subseteq \mathcal{E}_{\hat{\mathbf{U}}_{2}}^{\prime} \cap \mathcal{E}_{\mathbf{S}}^{c} \cap \mathcal{E}_{\mathbf{X}}^{c} \cap \mathcal{E}_{\mathbf{Z}}^{c}
$$

and, in particular

$$
\operatorname{Pr}\left[\mathcal{E}_{\hat{\mathbf{U}}_{2}} \cap \mathcal{E}_{\mathbf{S}}^{c} \cap \mathcal{E}_{\mathbf{X}}^{c} \cap \mathcal{E}_{\mathbf{Z}}^{c}\right] \leq \operatorname{Pr}\left[\mathcal{E}_{\hat{\mathbf{U}}_{2}}^{\prime} \cap \mathcal{E}_{\mathbf{S}}^{c} \cap \mathcal{E}_{\mathbf{X}}^{c} \cap \mathcal{E}_{\mathbf{Z}}^{c}\right] .
$$

Proof: We first recall that for the event $\mathcal{E}_{\hat{\mathbf{U}}_{2}}$ to occur, there must exist a codeword $\mathbf{u}_{2}(j) \in$ $\mathcal{C}_{2} \backslash\left\{\mathbf{u}_{2}^{*}\right\}$ that satisfies the following three conditions

$$
\begin{aligned}
\left|\tilde{\rho}-\cos \varangle\left(\mathbf{u}_{1}^{*}, \mathbf{u}_{2}(j)\right)\right| & \leq 7 \epsilon \\
\left|\bar{\rho}-\cos \varangle\left(\mathbf{v}^{*}, \mathbf{u}_{2}(j)\right)\right| & \leq 7 \epsilon \\
\left\|\mathbf{y}-\mathbf{X}_{\mathbf{u}_{1}^{*}, \mathbf{v}^{*}, \mathbf{u}_{2}(j)}\right\|^{2} & \leq\left\|\mathbf{y}-\mathbf{X}_{\mathbf{u}_{1}^{*}, \mathbf{v}^{*}, \mathbf{u}_{2}^{*}}\right\|^{2} .
\end{aligned}
$$

The proof is now based on a sequence of statements related to these three conditions.

A) For every $\left(\mathbf{s}_{1}, \mathbf{s}_{2}, \mathcal{C}_{1}, \mathcal{C}_{2}, \mathcal{C}_{c}, \mathbf{z}\right) \in \mathcal{E}_{\mathbf{X}}^{c}$ and every $\mathbf{u} \in \mathcal{S}_{2}$, where $\mathcal{S}_{2}$ is the surface area of the codeword sphere of $\mathcal{C}_{2}$ defined in the code construction,

$$
\begin{aligned}
& \left|\tilde{\rho}-\cos \varangle\left(\mathbf{u}_{1}^{*}, \mathbf{u}\right)\right| \leq 7 \epsilon \\
& \quad \Longrightarrow\left|n \tilde{\rho} \sqrt{\bar{\beta}_{1} \bar{\beta}_{2} P_{1} P_{2}}-\left\langle a_{1,1} \mathbf{u}_{1}^{*}, a_{2,1} \mathbf{u}\right\rangle\right| \leq 7 n \sqrt{\bar{\beta}_{1} \bar{\beta}_{2} P_{1} P_{2}} \epsilon .
\end{aligned}
$$

Statement A) follows by rewriting $\cos \varangle\left(\mathbf{u}_{1}^{*}, \mathbf{u}\right)$ as $\left\langle\mathbf{u}_{1}^{*}, \mathbf{u}\right\rangle /\left(\left\|\mathbf{u}_{1}^{*}\right\|\|\mathbf{u}\|\right)$, and then multiplying the inequality on the 1.h.s. of (131) by $\left\|a_{1,1} \mathbf{u}_{1}^{*}\right\| \cdot\left\|a_{2,1} \mathbf{u}\right\|$ and recalling that $\left\|a_{1,1} \mathbf{u}_{1}^{*}\right\|=\sqrt{n \bar{\beta}_{1} P_{1}}$ and that $\left\|a_{2,1} \mathbf{u}\right\|=\sqrt{n \bar{\beta}_{2} P_{2}}$.

A1) For every $\left(\mathbf{s}_{1}, \mathbf{s}_{2}, \mathcal{C}_{1}, \mathcal{C}_{2}, \mathcal{C}_{c}, \mathbf{z}\right) \in \mathcal{E}_{\mathbf{X}}^{c}$,

$$
\begin{aligned}
& \left|\cos \varangle\left(\mathbf{v}^{*}, \mathbf{u}_{1}^{*}\right)\right| \leq 3 \epsilon \\
& \Longrightarrow\left|\left\langle\alpha \mathbf{v}^{*}, a_{1,1} \mathbf{u}_{1}^{*}\right\rangle\right| \leq 3\left\|\alpha \mathbf{v}^{*}\right\| \sqrt{n \bar{\beta}_{1} P_{1}} \epsilon .
\end{aligned}
$$


Statement A1) follows by rewriting $\cos \varangle\left(\mathbf{v}^{*}, \mathbf{u}_{1}^{*}\right)$ as $\left\langle\mathbf{v}^{*}, \mathbf{u}_{1}^{*}\right\rangle /\left(\left\|\mathbf{v}^{*}\right\|\left\|\mathbf{u}_{1}^{*}\right\|\right)$, and then multiplying the inequality on the 1.h.s. of (132) by $\left\|\alpha \mathbf{v}^{*}\right\| \cdot\left\|a_{1,1} \mathbf{u}_{1}^{*}\right\|$.

A2) For every $\left(\mathbf{s}_{1}, \mathbf{s}_{2}, \mathcal{C}_{1}, \mathcal{C}_{2}, \mathcal{C}_{c}, \mathbf{z}\right) \in \mathcal{E}_{\mathbf{X}}^{c}$ and every $\mathbf{u} \in \mathcal{S}_{2}$,

$$
\begin{aligned}
& \left|\bar{\rho}-\cos \varangle\left(\mathbf{v}^{*}, \mathbf{u}\right)\right| \leq 7 \epsilon \\
& \quad \Longrightarrow\left|\left\|\alpha \mathbf{v}^{*}\right\| \sqrt{n \bar{\beta}_{2} P_{2}} \bar{\rho}-\left\langle\alpha \mathbf{v}^{*}, a_{2,1} \mathbf{u}\right\rangle\right| \leq 7 \epsilon\left\|\alpha \mathbf{v}^{*}\right\| \sqrt{n \bar{\beta}_{2} P_{2}} .
\end{aligned}
$$

Statement A2) follows by rewriting $\cos \varangle\left(\mathbf{v}^{*}, \mathbf{u}_{2}\right)$ as $\left\langle\mathbf{v}^{*}, \mathbf{u}_{2}\right\rangle /\left(\left\|\mathbf{v}^{*}\right\|\left\|\mathbf{u}_{2}\right\|\right)$, and then multiplying the inequality on the 1.h.s. of (133) by $\left\|\alpha \mathbf{v}^{*}\right\| \cdot\left\|a_{2,1} \mathbf{u}\right\|$.

B) For every $\left(\mathbf{s}_{1}, \mathbf{s}_{2}, \mathcal{C}_{1}, \mathcal{C}_{2}, \mathcal{C}_{c}, \mathbf{z}\right) \in \mathcal{E}_{\mathbf{X}}^{c} \cap \mathcal{E}_{\mathbf{Z}}^{c}$ and every $\mathbf{u} \in \mathcal{S}_{2}$

$$
\begin{aligned}
& \mid \mathbf{y}-\mathbf{X}_{\mathbf{u}_{1}^{*}, \mathbf{v}^{*}, \mathbf{u}}\left\|^{2} \leq\right\| \mathbf{y}-\mathbf{X}_{\mathbf{u}_{1}^{*}, \mathbf{v}^{*}, \mathbf{u}_{2}^{*}} \|^{2} \\
& \quad \Longrightarrow\left\langle a_{2,1} \mathbf{u}_{2}^{*}+\mathbf{z}, a_{2,1} \mathbf{u}\right\rangle \geq n\left(\bar{\beta}_{2} P_{2}-\sqrt{\bar{\beta}_{2} P_{2} N \epsilon}\right) .
\end{aligned}
$$

Statement B) follows from rewriting the inequality on the 1.h.s. of (134) as

$$
\left\|\left(\mathbf{y}-a_{1,1} \mathbf{u}_{1}^{*}-\alpha \mathbf{v}^{*}\right)-a_{2,1} \mathbf{u}\right\|^{2} \leq\left\|\left(\mathbf{y}-a_{1,1} \mathbf{u}_{1}^{*}-\alpha \mathbf{v}^{*}\right)-a_{2,1} \mathbf{u}_{2}^{*}\right\|^{2},
$$

or equivalently as

$$
\begin{aligned}
\left\langle\mathbf{y}-a_{1,1} \mathbf{u}_{1}^{*}-\alpha \mathbf{v}^{*}, a_{2,1} \mathbf{u}\right\rangle & \geq\left\langle\mathbf{y}-a_{1,1} \mathbf{u}_{1}^{*}-\alpha \mathbf{v}^{*}, a_{2,1} \mathbf{u}_{2}^{*}\right\rangle \\
& =\left\langle a_{2,1} \mathbf{u}_{2}^{*}+\mathbf{z}, a_{2,1} \mathbf{u}\right\rangle \\
& =\left\|a_{2,1} \mathbf{u}_{2}^{*}\right\|^{2}+\left\langle\mathbf{z}, a_{2,1} \mathbf{u}_{2}^{*}\right\rangle \\
& \geq n\left(\bar{\beta}_{2} P_{2}-\sqrt{\bar{\beta}_{2} P_{2} N \epsilon}\right)
\end{aligned}
$$

thus establishing B).

C) For every $\left(\mathbf{s}_{1}, \mathbf{s}_{2}, \mathcal{C}_{1}, \mathcal{C}_{2}, \mathcal{C}_{c}, \mathbf{z}\right) \in \mathcal{E}_{\mathbf{X}}^{c} \cap \mathcal{E}_{\mathbf{Z}}^{c}$ and every $\mathbf{u} \in \mathcal{S}_{2}$,

$$
\begin{gathered}
\left|\tilde{\rho}-\cos \varangle\left(\mathbf{u}_{1}^{*}, \mathbf{u}\right)\right| \leq 7 \epsilon \text { and }\left|\bar{\rho}-\cos \varangle\left(\mathbf{u}_{2}, \mathbf{v}^{*}\right)\right| \leq 7 \epsilon \text { and } \mid \mathbf{y}-\mathbf{X}_{\mathbf{u}_{1}^{*}, \mathbf{v}^{*}, \mathbf{u}}\left\|^{2} \leq\right\| \mathbf{y}-\mathbf{X}_{\mathbf{u}_{1}^{*}, \mathbf{v}^{*}, \mathbf{u}_{2}^{*}} \|^{2} \\
\Longrightarrow \\
\left\|a_{2,1} \mathbf{u}-\mathbf{w}\right\|^{2} \leq n \bar{\beta}_{2} P_{2}-2\left(n \varsigma_{1}\left(\bar{\beta}_{2} P_{2}-\sqrt{\bar{\beta}_{2} P_{2} N \epsilon}\right)+n \varsigma_{2}\left(\sqrt{\bar{\beta}_{1} \bar{\beta}_{2} P_{1} P_{2}}(\tilde{\rho}-7 \epsilon)\right)\right. \\
\left.+n \varsigma_{3}\left(\frac{1}{n}\left\|\alpha \mathbf{v}^{*}\right\| \sqrt{n \bar{\beta}_{2} P_{2}}(\bar{\rho}-7 \epsilon)\right)\right)+\|\mathbf{w}\|^{2} .
\end{gathered}
$$

Statement C) is obtained as follows:

$$
\begin{aligned}
& \left\|a_{2,1} \mathbf{u}-\mathbf{w}\right\|^{2} \quad=\left\|a_{2,1} \mathbf{u}\right\|^{2}-2\left\langle a_{2,1} \mathbf{u}, \mathbf{w}\right\rangle+\|\mathbf{w}\|^{2} \\
& =\left\|a_{2,1} \mathbf{u}\right\|^{2}-2\left\langle a_{2,1} \mathbf{u}, \varsigma_{1}\left(a_{2,1} \mathbf{u}_{2}^{*}+\mathbf{z}\right)+\varsigma_{2} a_{1,1} \mathbf{u}_{1}^{*}+\varsigma_{3} \alpha \mathbf{v}^{*}\right\rangle+\|\mathbf{w}\|^{2} \\
& =n \bar{\beta}_{2} P_{2}-2\left[\varsigma_{1}\left\langle a_{2,1} \mathbf{u}, a_{2,1} \mathbf{u}_{2}^{*}+\mathbf{z}\right\rangle+\varsigma_{2}\left\langle a_{2,1} \mathbf{u}, a_{1,1} \mathbf{u}_{1}^{*}\right\rangle+\varsigma_{3}\left\langle a_{2,1} \mathbf{u}, \alpha \mathbf{v}^{*}\right\rangle\right]+\|\mathbf{w}\|^{2} \\
& \stackrel{(a)}{\leq} n \bar{\beta}_{2} P_{2}-2 n\left(\varsigma_{1}\left(\bar{\beta}_{2} P_{2}-\sqrt{\bar{\beta}_{2} P_{2} N \epsilon}\right)+\varsigma_{2}\left(\sqrt{\bar{\beta}_{1} \bar{\beta}_{2} P_{1} P_{2}}(\tilde{\rho}-7 \epsilon)\right)\right. \\
& \left.\quad+\varsigma_{3}\left(\frac{1}{n}\left\|\alpha \mathbf{v}^{*}\right\| \sqrt{n \bar{\beta}_{2} P_{2}}(\bar{\rho}-7 \epsilon)\right)\right)+\|\mathbf{w}\|^{2},
\end{aligned}
$$

where in (a) we have used Statement A), Statement A2) and Statement B). 
D) For every $\left(\mathbf{s}_{1}, \mathbf{s}_{2}, \mathcal{C}_{1}, \mathcal{C}_{2}, \mathcal{C}_{c}, \mathbf{z}\right) \in \mathcal{E}_{\mathbf{X}}^{c} \cap \mathcal{E}_{\mathbf{Z}}^{c}$

$$
\begin{aligned}
\|\mathbf{w}\|^{2} \leq & n\left(\varsigma_{1}{ }^{2}\left(\bar{\beta}_{2} P_{2}+N\right)+2 \varsigma_{1} \varsigma_{2} \sqrt{\bar{\beta}_{1} \bar{\beta}_{2} P_{1} P_{2}} \tilde{\rho}+\varsigma_{2}{ }^{2}\left(\bar{\beta}_{1} P_{1}\right)\right. \\
& \left.+\frac{1}{n} 2 \varsigma_{1} \varsigma_{3}\left\|\alpha \mathbf{v}^{*}\right\| \sqrt{n \bar{\beta}_{2} P_{2}} \bar{\rho}+\frac{1}{n} \varsigma_{3}{ }^{2} \alpha^{2}\left\|\mathbf{v}^{*}\right\|^{2}+\kappa \epsilon\right),
\end{aligned}
$$

where $\kappa$ depends on $P_{1}, P_{2}, N, \varsigma_{1}, \varsigma_{2}$ and $\varsigma_{3}$ only.

Statement D) is obtained as follows

$$
\begin{aligned}
\|\mathbf{w}\|^{2}= & \left\|\varsigma_{1}\left(a_{2,1} \mathbf{u}_{2}^{*}+\mathbf{z}\right)+\varsigma_{2} a_{1,1} \mathbf{u}_{1}^{*}+\varsigma_{3} \alpha \mathbf{v}^{*}\right\|^{2} \\
= & \varsigma_{1}{ }^{2}\left\|a_{2,1} \mathbf{u}_{2}^{*}+\mathbf{z}\right\|^{2}+2 \varsigma_{1} \varsigma_{2}\left\langle a_{2,1} \mathbf{u}_{2}^{*}+\mathbf{z}, a_{1,1} \mathbf{u}_{1}^{*}\right\rangle+\varsigma_{2}{ }^{2}\left\|a_{1,1} \mathbf{u}_{1}^{*}\right\|^{2}+2 \varsigma_{1} \varsigma_{3}\left\langle a_{2,1} \mathbf{u}_{2}^{*}+\mathbf{z}, \alpha \mathbf{v}^{*}\right\rangle \\
& +2 \varsigma_{2} \varsigma_{3}\left\langle a_{1,1} \mathbf{u}_{1}^{*}, \alpha \mathbf{v}^{*}\right\rangle+\varsigma_{3}{ }^{2}\left\|\alpha \mathbf{v}^{*}\right\|^{2} \\
= & \varsigma_{1}{ }^{2}\left(\left\|a_{2,1} \mathbf{u}_{2}^{*}\right\|^{2}+2\left\langle a_{2,1} \mathbf{u}_{2}^{*}, \mathbf{z}\right\rangle+\|\mathbf{z}\|^{2}\right)+2 \varsigma_{1} \varsigma_{2}\left(\left\langle a_{2,1} \mathbf{u}_{2}^{*}, a_{1,1} \mathbf{u}_{1}^{*}\right\rangle+\left\langle\mathbf{z}, a_{1,1} \mathbf{u}_{1}^{*}\right\rangle\right) \\
& +\varsigma_{2}{ }^{2}\left(n \bar{\beta}_{1} P_{1}\right)+2 \varsigma_{1} \varsigma_{3}\left[\left\langle a_{2,1} \mathbf{u}_{2}^{*}, \alpha \mathbf{v}^{*}\right\rangle+\left\langle\mathbf{z}, \alpha \mathbf{v}^{*}\right\rangle\right]+2 \varsigma_{2} \varsigma_{3}\left\langle a_{1,1} \mathbf{u}_{1}^{*}, \alpha \mathbf{v}^{*}\right\rangle+\varsigma_{3}{ }^{2} \alpha^{2}\left\|\mathbf{v}^{*}\right\|^{2} \\
(a) & n\left(\varsigma_{1}^{2}\left(\bar{\beta}_{2} P_{2}+N\right)+2 \varsigma_{1} \varsigma_{2} \sqrt{\bar{\beta}_{1} \bar{\beta}_{2} P_{1} P_{2}} \tilde{\rho}+\varsigma_{2}{ }^{2}\left(\bar{\beta}_{1} P_{1}\right)+\frac{1}{n} 2 \varsigma_{1} \varsigma_{3}\left\|\alpha \mathbf{v}^{*}\right\| \sqrt{n \bar{\beta}_{2} P_{2}} \bar{\rho}\right. \\
& \left.+\frac{1}{n} \varsigma_{3}{ }^{2} \alpha^{2}\left\|\mathbf{v}^{*}\right\|^{2}+k \epsilon\right),
\end{aligned}
$$

where in (a) we have used that $\left(\mathbf{s}_{1}, \mathbf{s}_{2}, \mathcal{C}_{1}, \mathcal{C}_{2}, \mathcal{C}_{c}, \mathbf{z}\right) \in \mathcal{E}_{\mathbf{X}}^{c}$, and statements A),A1) and A2).

E) For every $\left(\mathbf{s}_{1}, \mathbf{s}_{2}, \mathcal{C}_{1}, \mathcal{C}_{2}, \mathcal{C}_{c}, \mathbf{z}\right) \in \mathcal{E}_{\mathbf{X}}^{c} \cap \mathcal{E}_{\mathbf{Z}}^{c}$ and an arbitrary $\mathbf{u} \in \mathcal{S}_{2}$,

$$
\begin{aligned}
& \left|\tilde{\rho}-\cos \varangle\left(\mathbf{u}_{1}^{*}, \mathbf{u}\right)\right| \leq 7 \epsilon \text { and }\left|\bar{\rho}-\cos \varangle\left(\mathbf{v}^{*}, \mathbf{u}\right)\right| \leq 7 \epsilon \text { and } \mid \mathbf{y}-\mathbf{X}_{\mathbf{u}_{1}^{*}, \mathbf{v}^{*}, \mathbf{u}}\left\|^{2} \leq\right\| \mathbf{y}-\mathbf{X}_{\mathbf{u}_{1}^{*}, \mathbf{v}^{*}, \mathbf{u}_{2}^{*}} \|^{2} \\
& \Longrightarrow\left\|a_{2,1} \mathbf{u}-\mathbf{w}\right\|^{2} \leq \Upsilon(\epsilon),
\end{aligned}
$$

where

$$
\Upsilon(\epsilon)=n\left(\frac{\bar{\beta}_{2} P_{2} N\left(1-\bar{\rho}^{2}-\tilde{\rho}^{2}\right)}{\bar{\beta}_{2} P_{2}\left(1-\bar{\rho}^{2}-\tilde{\rho}^{2}\right)+N}+\frac{\bar{\beta}_{2} P_{2} N^{2} \tilde{\rho}^{2} \bar{\rho}^{2}\left(2+\tilde{\rho}^{2}\right)}{\left(\bar{\beta}_{2} P_{2}\left(1-\bar{\rho}^{2}-\tilde{\rho}^{2}\right)+N\right)^{2}}\right)+n \kappa^{\prime} \epsilon,
$$

and where $\kappa^{\prime}$ only depends on $P_{2}, N_{1}, N_{2}, \varsigma_{1}, \varsigma_{2}$ and $\varsigma_{3}$.

Statement E) follows from combining Statement C) with Statement D) and the explicit values of $\varsigma_{1}, \varsigma_{2}$ and $\varsigma_{3}$ given in (126).

F) For every $\mathbf{u} \in \mathcal{S}_{2}$, denote by $\varphi \in[0, \pi]$ the angle between $\mathbf{u}$ and $\mathbf{w}$, and let

$$
\begin{aligned}
& \mathcal{B}\left(\mathbf{s}_{1}, \mathbf{s}_{2}, \mathbf{u}_{1}^{*}, \mathbf{v}^{*}, \mathbf{u}_{2}^{*}, \mathbf{z}\right) \triangleq\left\{\mathbf{u} \in S_{2}^{(n)}:\right. \\
& \cos (\varphi) \geq \sqrt{\left.\frac{\bar{\beta}_{2} P_{2}\left[1-\tilde{\rho}^{2}\right]+N \tilde{\rho}^{2}-\bar{\rho}^{2}\left(\bar{\beta}_{2} P_{2}-N\right)}{\bar{\beta}_{2} P_{2}\left(1-\tilde{\rho}^{2}\right)+N-\bar{\rho}^{2} \bar{\beta}_{2} P_{2}}-\frac{N^{2} \tilde{\rho}^{2} \bar{\rho}^{2}\left(2+\tilde{\rho}^{2}\right)}{\left(\bar{\beta}_{2} P_{2}\left[1-\bar{\rho}^{2}-\tilde{\rho}^{2}\right]+N\right)^{2}}-\kappa^{\prime \prime} \epsilon\right\}},
\end{aligned}
$$

where $\kappa^{\prime \prime}$ only depends on $P_{1}, N_{1}, N_{2}, \varsigma_{1}, \varsigma_{2}$ and $\varsigma_{3}$.

Then, for every $\left(\mathbf{s}_{1}, \mathbf{s}_{2}, \mathcal{C}_{1}, \mathcal{C}_{2}, \mathcal{C}_{c}, \mathbf{z}\right) \in \mathcal{E}_{\mathbf{X}}^{c} \cap \mathcal{E}_{\mathbf{Z}}^{c}$,

$$
\begin{aligned}
& \left|\tilde{\rho}-\cos \varangle\left(\mathbf{u}_{1}^{*}, \mathbf{u}\right)\right| \leq 7 \epsilon \quad \text { and } \quad\left|\bar{\rho}-\cos \varangle\left(\mathbf{v}^{*}, \mathbf{u}\right)\right| \leq 7 \epsilon \quad \text { and } \\
& \left\|\mathbf{y}-\left(a_{1,1} \mathbf{u}_{1}^{*}+a_{2,1} \mathbf{u}+\alpha \mathbf{v}^{*}\right)\right\|^{2} \leq\left\|\mathbf{y}-\left(a_{1,1} \mathbf{u}_{1}^{*}+a_{2,1} \mathbf{u}_{2}^{*}+\alpha \mathbf{v}^{*}\right)\right\|^{2} \\
& \Longrightarrow \mathbf{u} \in \mathcal{B}\left(\mathbf{s}_{1}, \mathbf{s}_{2}, \mathbf{u}_{1}^{*}, \mathbf{v}^{*}, \mathbf{u}_{2}^{*}, \mathbf{z}\right) .
\end{aligned}
$$


Statement F) follows from Statement E) by noting that if $\mathbf{w} \neq 0$ and $1-\frac{\Upsilon(\epsilon)}{n \beta_{2} P_{2}}>0$, then

$$
\left.\begin{array}{l}
\left\|a_{2,1} \mathbf{u}\right\|^{2}=n \bar{\beta}_{2} P_{2} \\
\left\|a_{2,1} \mathbf{u}-\mathbf{w}\right\|^{2} \leq \Upsilon(\epsilon)
\end{array}\right\} \Longrightarrow \cos \varangle(\mathbf{u}, \mathbf{w}) \geq \sqrt{1-\frac{\Upsilon(\epsilon)}{n \bar{\beta}_{2} P_{2}}},
$$

which follows by the same argument as (123).

The proof of Lemma 17 is now concluded by noticing that the set $\mathcal{E}_{\hat{\mathbf{U}}_{2}}^{\prime}$, defined in 127], is the set of tuples $\left(\mathbf{s}_{1}, \mathbf{s}_{2}, \mathcal{C}_{1}, \mathcal{C}_{2}, \mathcal{C}_{c}, \mathbf{z}\right)$ for which there exists a $\mathbf{u}_{2}(j) \in \mathcal{C}_{2} \backslash\left\{\mathbf{u}_{2}^{*}\right\}$ such that $\mathbf{u}_{2}(j) \in \mathcal{B}\left(\mathbf{s}_{1}, \mathbf{s}_{2}, \mathbf{u}_{1}^{*}, \mathbf{v}^{*}, \mathbf{u}_{2}^{*}, \mathbf{z}\right)$. Thus, by Statement F) and by the definition of $\mathcal{E}_{\hat{\mathbf{U}}_{2}}$ in 88) it follows that

$$
\mathcal{E}_{\hat{\mathbf{U}}_{2}} \cap \mathcal{E}_{\mathbf{S}}^{c} \cap \mathcal{E}_{\mathbf{X}}^{c} \cap \mathcal{E}_{\mathbf{Z}}^{c} \subseteq \mathcal{E}_{\hat{\mathbf{U}}_{2}}^{\prime} \cap \mathcal{E}_{\mathbf{S}}^{c} \cap \mathcal{E}_{\mathbf{X}}^{c} \cap \mathcal{E}_{\mathbf{Z}}^{c}
$$

and therefore

$$
\operatorname{Pr}\left[\mathcal{E}_{\hat{\mathbf{U}}_{2}} \cap \mathcal{E}_{\mathbf{S}}^{c} \cap \mathcal{E}_{\mathbf{X}}^{c} \cap \mathcal{E}_{\mathbf{Z}}^{c}\right] \leq \operatorname{Pr}\left[\mathcal{E}_{\hat{\mathbf{U}}_{2}}^{\prime} \cap \mathcal{E}_{\mathbf{S}}^{c} \cap \mathcal{E}_{\mathbf{X}}^{c} \cap \mathcal{E}_{\mathbf{Z}}^{c}\right]
$$

Next,

$$
\begin{aligned}
\operatorname{Pr}\left[\mathcal{E}_{\hat{\mathbf{U}}_{2}} \cap \mathcal{E}_{\mathbf{S}}^{c} \cap \mathcal{E}_{\mathbf{X}}^{c} \cap \mathcal{E}_{\mathbf{Z}}^{c}\right] \stackrel{(a)}{\leq} \operatorname{Pr}\left[\mathcal{E}_{\hat{\mathbf{U}}_{2}}^{\prime} \cap \mathcal{E}_{\mathbf{S}}^{c} \cap \mathcal{E}_{\mathbf{X}}^{c} \cap \mathcal{E}_{\mathbf{Z}}^{c}\right] \\
\stackrel{(b)}{\leq} \operatorname{Pr}\left[\mathcal{E}_{\hat{\mathbf{U}}_{2}}^{\prime} \mid \mathcal{E}_{\mathbf{X}_{1}}^{c}\right],
\end{aligned}
$$

where (a) follows by Lemma 17 and (b) follows because $\mathcal{E}_{\mathbf{X}}^{c} \subseteq \mathcal{E}_{\mathbf{X}_{2}}^{c}$. The proof of (103) is now completed by combining (137) with Lemma 16, This gives that for every $\delta>0$ and every $\epsilon>0$ there exists some $n^{\prime}(\delta, \epsilon)$ such that for all $n>n^{\prime}(\delta, \epsilon)$, we have

$$
\operatorname{Pr}\left[\mathcal{E}_{\hat{\mathbf{U}}_{2}} \cap \mathcal{E}_{\mathbf{S}}^{c} \cap \mathcal{E}_{\mathbf{X}}^{c} \cap \mathcal{E}_{\mathbf{Z}}^{c}\right] \leq \operatorname{Pr}\left[\mathcal{E}_{\hat{\mathbf{U}}_{2}}^{\prime} \mid \mathcal{E}_{\mathbf{X}_{2}}^{c}\right]<\delta,
$$

whenever

$$
R_{2}<\frac{1}{2} \log \left(\frac{\bar{\beta}_{2} P_{2}\left(1-\tilde{\rho}^{2}-\bar{\rho}^{2}\right)+N}{N\left(1-\tilde{\rho}^{2}-\bar{\rho}^{2}\right)+\lambda_{2}}-\kappa_{2} \epsilon\right),
$$

where $\kappa_{2}$ is a positive constant determined by $P_{1}, P_{2}, N, \varsigma_{1}, \varsigma_{2}$ and $\varsigma_{3}$.

\section{Proof of rate constraint (104)}

Define

$$
\mathbf{w}\left(\mathbf{s}_{1}, \mathbf{s}_{2}, \mathcal{C}_{1}, \mathcal{C}_{2}, \mathcal{C}_{c}, \mathbf{z}\right)=\varsigma_{1}\left(\mathbf{y}-\left(a_{1,1} \mathbf{u}_{1}^{*}+a_{2,1} \mathbf{u}_{2}^{*}\right)\right)+\varsigma_{2} a_{1,1} \mathbf{u}_{1}^{*}+\varsigma_{3} a_{2,1} \mathbf{u}_{2}^{*},
$$

where

$$
\begin{aligned}
& \varsigma_{1}=\frac{\sigma^{2} \alpha^{2}\left(1-2^{-2 R_{\mathrm{c}}}\right) 2^{-2 R_{1}}\left(1-\tilde{\rho}^{2}-\bar{\rho}^{2}\right)}{\sigma^{2} \alpha^{2}\left(1-2^{-2 R_{\mathrm{c}}}\right) 2^{-2 R_{1}}\left(1-\tilde{\rho}^{2}-\bar{\rho}^{2}\right)+N\left(1-\tilde{\rho}^{2}\right)} \\
& \varsigma_{2}=-\frac{\alpha \bar{\rho}^{2} N}{a_{1,1}\left(\sigma^{2} \alpha^{2}\left(1-2^{-2 R_{\mathrm{c}}}\right) 2^{-2 R_{1}}\left(1-\tilde{\rho}^{2}-\bar{\rho}^{2}\right)+N\left(1-\tilde{\rho}^{2}\right)\right)} \\
& \varsigma_{3}=\frac{\alpha \rho\left(1-2^{-2 R_{\mathrm{c}}}\right) 2^{-2 R_{1}} N}{a_{2,1}\left(\sigma^{2} \alpha^{2}\left(1-2^{-2 R_{\mathrm{c}}}\right) 2^{-2 R_{1}}\left(1-\tilde{\rho}^{2}-\bar{\rho}^{2}\right)+N\left(1-\tilde{\rho}^{2}\right)\right)} .
\end{aligned}
$$

We now start with a lemma that will be used to prove (104). 
Lemma 18 Let $\varphi_{j} \in[0, \pi]$ be the angle between $\mathbf{w}$ and $\mathbf{v}(j)$, and let the set $\mathcal{E}_{\hat{\mathbf{v}}}^{\prime}$ be defined as

$$
\begin{array}{r}
\mathcal{E}_{\hat{\mathbf{v}}}^{\prime} \triangleq\left\{\left(\mathbf{s}_{1}, \mathbf{s}_{2}, \mathcal{C}_{1}, \mathcal{C}_{2}, \mathcal{C}_{c}, \mathbf{z}\right): \exists \mathbf{v}(j) \in \mathcal{C}_{c} \backslash\left\{\mathbf{v}^{*}\right\}\right. \text { s.t. } \\
\left.\quad \cos \left(\varphi_{j}\right) \geq \sqrt{1-\frac{\Upsilon(\epsilon)}{n \alpha^{2} \sigma^{2} 2^{-2 R_{1}}\left(1-2^{-2 R_{\mathrm{c}}}\right)}}\right\},
\end{array}
$$

where $\Upsilon(\epsilon)$ is defined in 148. Then,

$$
\mathcal{E}_{\hat{\mathbf{V}}} \cap \mathcal{E}_{\mathbf{S}}^{c} \cap \mathcal{E}_{\mathbf{X}}^{c} \cap \mathcal{E}_{\mathbf{Z}}^{c} \subseteq \mathcal{E}_{\hat{\mathbf{V}}}^{\prime} \cap \mathcal{E}_{\mathbf{S}}^{c} \cap \mathcal{E}_{\mathbf{X}}^{c} \cap \mathcal{E}_{\mathbf{Z}}^{c},
$$

and, in particular

$$
\operatorname{Pr}\left[\mathcal{E}_{\hat{\mathbf{V}}} \cap \mathcal{E}_{\mathbf{S}}^{c} \cap \mathcal{E}_{\mathbf{X}}^{c} \cap \mathcal{E}_{\mathbf{Z}}^{c}\right] \leq \operatorname{Pr}\left[\mathcal{E}_{\hat{\mathbf{V}}}^{\prime} \cap \mathcal{E}_{\mathbf{S}}^{c} \cap \mathcal{E}_{\mathbf{X}}^{c} \cap \mathcal{E}_{\mathbf{Z}}^{c}\right] .
$$

Proof: We first recall that for the event $\mathcal{E}_{\hat{\mathbf{v}}}$ to occur, there must exist a codeword $\mathbf{v}(j) \in$ $\mathcal{C}_{c} \backslash\left\{\mathbf{v}^{*}\right\}$ that satisfies the following three conditions

$$
\begin{aligned}
\left|\bar{\rho}-\cos \varangle\left(\mathbf{v}(j), \mathbf{u}_{2}^{*}\right)\right| & \leq 7 \epsilon \\
\left|\cos \varangle\left(\mathbf{v}(j), \mathbf{u}_{1}^{*}\right)\right| & \leq 3 \epsilon \\
\left\|\mathbf{y}-\mathbf{X}_{\mathbf{u}_{1}^{*}, \mathbf{v}(j), \mathbf{u}_{2}^{*}}\right\|^{2} & \leq\left\|\mathbf{y}-\mathbf{X}_{\mathbf{u}_{1}^{*}, \mathbf{v}^{*}, \mathbf{u}_{2}^{*}}\right\|^{2} .
\end{aligned}
$$

The proof is now based on a sequence of statements related to these three conditions.

A) For every $\left(\mathbf{s}_{1}, \mathbf{s}_{2}, \mathcal{C}_{1}, \mathcal{C}_{2}, \mathcal{C}_{c}, \mathbf{z}\right) \in \mathcal{E}_{\mathbf{X}}^{c}$ and every $\mathbf{v} \in \mathcal{S}_{c}$, where $S_{c}$ is the surface area of the codeword sphere of $\mathcal{C}_{c}$ defined in the code construction,

$$
\begin{aligned}
& \left|\bar{\rho}-\cos \varangle\left(\mathbf{v}, \mathbf{u}_{2}^{*}\right)\right| \leq 7 \epsilon \\
& \Longrightarrow\left|\left\|\alpha \mathbf{v}^{*}\right\| \sqrt{n \bar{\beta}_{2} P_{2}} \bar{\rho}-\left\langle\alpha \mathbf{v}, a_{2,1} \mathbf{u}_{2}^{*}\right\rangle\right| \leq\left\|\alpha \mathbf{v}^{*}\right\| \sqrt{n \bar{\beta}_{2} P_{2}} 7 \epsilon .
\end{aligned}
$$

Statement A) follows by rewriting $\cos \varangle\left(\mathbf{v}, \mathbf{u}_{2}^{*}\right)$ as $\left\langle\mathbf{v}, \mathbf{u}_{2}^{*}\right\rangle /\left(\|\mathbf{v}\|\left\|\mathbf{u}_{2}^{*}\right\|\right)$, and then multiplying the inequality on the 1.h.s. of (144) by $\left\|\alpha \mathbf{v}^{*}\right\| \cdot\left\|a_{2,1} \mathbf{u}_{2}^{*}\right\|$.

A1) For every $\left(\mathbf{s}_{1}, \mathbf{s}_{2}, \mathcal{C}_{1}, \mathcal{C}_{2}, \mathcal{C}_{c}, \mathbf{z}\right) \in \mathcal{E}_{\mathbf{X}}^{c}$,

$$
\begin{aligned}
& \left|\cos \varangle\left(\mathbf{v}, \mathbf{u}_{1}^{*}\right)\right| \leq 3 \epsilon \\
& \quad \Longrightarrow \quad\left|\left\langle\alpha \mathbf{v}, a_{1,1} \mathbf{u}_{1}^{*}\right\rangle\right| \leq 3\|\alpha \mathbf{v}\| \sqrt{n \bar{\beta}_{1} P_{1}} \epsilon .
\end{aligned}
$$

Statement A1) follows by rewriting $\cos \varangle\left(\mathbf{v}, \mathbf{u}_{1}^{*}\right)$ as $\left\langle\mathbf{v}, \mathbf{u}_{1}^{*}\right\rangle /\left(\left\|\mathbf{v}^{*}\right\|\left\|\mathbf{u}_{1}^{*}\right\|\right)$, and then multiplying the inequality on the 1.h.s. of (145) by $\|\alpha \mathbf{v}\| \cdot\left\|a_{1,1} \mathbf{u}_{1}^{*}\right\|$ and recalling that $\left\|a_{1,1} \mathbf{u}_{1}^{*}\right\|=\sqrt{n \bar{\beta}_{1} P_{1}}$.

B) For every $\left(\mathbf{s}_{1}, \mathbf{s}_{2}, \mathcal{C}_{1}, \mathcal{C}_{2}, \mathcal{C}_{c}, \mathbf{z}\right) \in \mathcal{E}_{\mathbf{X}}^{c} \cap \mathcal{E}_{\mathbf{Z}}^{c}$ and every $\mathbf{v} \in \mathcal{S}_{c}$

$$
\begin{aligned}
& \mid \mathbf{y}-\mathbf{X}_{\mathbf{u}_{1}^{*}, \mathbf{v}, \mathbf{u}_{2}^{*}}\left\|^{2} \leq\right\| \mathbf{y}-\mathbf{X}_{\mathbf{u}_{1}^{*}, \mathbf{v}^{*}, \mathbf{u}_{2}^{*}} \|^{2} \\
& \quad \Longrightarrow\left\langle\mathbf{y}-\left(a_{1,1} \mathbf{u}_{1}^{*}-a_{2,1} \mathbf{u}_{2}^{*}\right), \alpha \mathbf{v}\right\rangle \geq\left\|\alpha \mathbf{v}^{*}\right\|^{2}-\left\|\alpha \mathbf{v}^{*}\right\| \sqrt{n N} \epsilon .
\end{aligned}
$$

Statement B) follows from rewriting the inequality on the 1.h.s. of (146) as

$$
\left\|\left(\mathbf{y}-a_{1,1} \mathbf{u}_{1}^{*}-a_{2,1} \mathbf{u}_{2}^{*}\right)-\alpha \mathbf{v}\right\|^{2} \leq\left\|\left(\mathbf{y}-a_{1,1} \mathbf{u}_{1}^{*}-a_{2,1} \mathbf{u}_{2}^{*}\right)-\alpha \mathbf{v}^{*}\right\|^{2},
$$

or equivalently as

$$
\begin{aligned}
\left\langle\mathbf{y}-a_{1,1} \mathbf{u}_{1}^{*}-a_{2,1} \mathbf{u}_{2}^{*}, \alpha \mathbf{v}\right\rangle & \geq\left\langle\mathbf{y}-a_{1,1} \mathbf{u}_{1}^{*}-a_{2,1} \mathbf{u}_{2}^{*}, \alpha \mathbf{v}^{*}\right\rangle \\
& =\left\langle\alpha \mathbf{v}^{*}+\mathbf{z}, \alpha \mathbf{v}^{*}\right\rangle \\
& =\left\|\alpha \mathbf{v}^{*}\right\|^{2}+\left\langle\mathbf{z}, \alpha \mathbf{v}^{*}\right\rangle
\end{aligned}
$$




$$
\geq\left\|\alpha \mathbf{v}^{*}\right\|^{2}-\left\|\alpha \mathbf{v}^{*}\right\| \sqrt{n N} \epsilon
$$

thus establising B).

C) For every $\left(\mathbf{s}_{1}, \mathbf{s}_{2}, \mathcal{C}_{1}, \mathcal{C}_{2}, \mathcal{C}_{c}, \mathbf{z}\right) \in \mathcal{E}_{\mathbf{X}}^{c} \cap \mathcal{E}_{\mathbf{Z}}^{c}$ and every $\mathbf{v} \in \mathcal{S}_{c}$,

$$
\begin{aligned}
& \left|\bar{\rho}-\cos \varangle\left(\mathbf{v}, \mathbf{u}_{2}^{*}\right)\right| \leq 7 \epsilon \text { and }\left|\cos \varangle\left(\mathbf{v}, \mathbf{u}_{1}^{*}\right)\right| \leq 3 \epsilon \text { and } \mid \mathbf{y}-\mathbf{X}_{\mathbf{u}_{1}^{*}, \mathbf{v}, \mathbf{u}_{2}^{*}}\left\|^{2} \leq\right\| \mathbf{y}-\mathbf{X}_{\mathbf{u}_{1}^{*}, \mathbf{v}^{*}, \mathbf{u}_{2}^{*}} \|^{2} \\
& \Longrightarrow \alpha \mathbf{v}-\mathbf{w}\left\|^{2} \leq\right\| \alpha \mathbf{v} \|^{2}-2 n\left(\varsigma_{1}\left(\frac{1}{n}\left\|\alpha \mathbf{v}^{*}\right\|^{2}-\frac{1}{n}\left\|\alpha \mathbf{v}^{*}\right\| \sqrt{n N} \epsilon\right)-\varsigma_{2}\|\alpha \mathbf{v}\| \sqrt{n \bar{\beta}_{1} P_{1}} 3 \epsilon\right. \\
& \left.+\varsigma_{3}\left(\frac{1}{n}\left\|\alpha \mathbf{v}^{*}\right\| \sqrt{n \bar{\beta}_{2} P_{2}}(\bar{\rho}-7 \epsilon)\right)\right)+\|\mathbf{w}\|^{2} .
\end{aligned}
$$

Statement C) is obtained as follows:

$$
\begin{aligned}
\|\alpha \mathbf{v}-\mathbf{w}\|^{2}= & \|\alpha \mathbf{v}\|^{2}-2\langle\alpha \mathbf{v}, \mathbf{w}\rangle+\|\mathbf{w}\|^{2} \\
= & \|\alpha \mathbf{v}\|^{2}-2\left\langle\alpha \mathbf{v}, \varsigma_{1}\left(\alpha \mathbf{v}^{*}+\mathbf{z}\right)+\varsigma_{2} a_{1,1} \mathbf{u}_{1}^{*}+\varsigma_{3} a_{2,1} \mathbf{u}_{2}^{*}\right\rangle+\|\mathbf{w}\|^{2} \\
= & \|\alpha \mathbf{v}\|^{2}-2\left(\varsigma_{1}\left\langle\alpha \mathbf{v}, \alpha \mathbf{v}^{*}+\mathbf{z}\right\rangle+\varsigma_{2}\left\langle\alpha \mathbf{v}, a_{1,1} \mathbf{u}_{1}^{*}\right\rangle\right. \\
& \left.+\varsigma_{3}\left\langle\alpha \mathbf{v}, a_{2,1} \mathbf{u}_{2}^{*}\right\rangle\right)+\|\mathbf{w}\|^{2} \\
\stackrel{(a)}{\leq} & \|\alpha \mathbf{v}\|^{2}-2\left(n \varsigma_{1}\left(\frac{1}{n}\left\|\alpha \mathbf{v}^{*}\right\|^{2}-\frac{1}{n}\left\|\alpha \mathbf{v}^{*}\right\| \sqrt{n N} \epsilon\right)-\varsigma_{2}\|\alpha \mathbf{v}\| \sqrt{n \bar{\beta}_{1} P_{1}} 3 \epsilon\right. \\
& \left.+n \varsigma_{3}\left(\frac{1}{n}\left\|\alpha \mathbf{v}^{*}\right\| \sqrt{n \bar{\beta}_{2} P_{2}}(\bar{\rho}-7 \epsilon)\right)\right)+\|\mathbf{w}\|^{2},
\end{aligned}
$$

where in (a) we have used Statement A), A1) and Statement B).

D) For every $\left(\mathbf{s}_{1}, \mathbf{s}_{2}, \mathcal{C}_{1}, \mathcal{C}_{2}, \mathcal{C}_{c}, \mathbf{z}\right) \in \mathcal{E}_{\mathbf{X}}^{c} \cap \mathcal{E}_{\mathbf{Z}}^{c}$

$$
\begin{aligned}
\|\mathbf{w}\|^{2} \leq & n\left(\frac{1}{n} \varsigma_{1}^{2}\left\|\alpha \mathbf{v}^{*}\right\|^{2}+\frac{2}{n} \varsigma_{1} \varsigma_{3}\left\|\alpha \mathbf{v}^{*}\right\| \sqrt{n \bar{\beta}_{2} P_{2}} \bar{\rho}+\varsigma_{1}{ }^{2} N+\varsigma_{2}{ }^{2} \bar{\beta}_{1} P_{1}\right. \\
& \left.+2 \varsigma_{2} \varsigma_{3} \sqrt{\bar{\beta}_{1} \bar{\beta}_{2} P_{1} P_{2}} \tilde{\rho}+\varsigma_{3}{ }^{2} \bar{\beta}_{2} P_{2}+\kappa \epsilon\right),
\end{aligned}
$$

where $\kappa$ depends on $P_{1}, P_{2}, N, \varsigma_{1}, \varsigma_{2}$ and $\varsigma_{3}$ only.

Statement D) is obtained as follows

$$
\begin{aligned}
& \|\mathbf{w}\|^{2}=\left\|\varsigma_{1}\left(\alpha \mathbf{v}^{*}+\mathbf{z}\right)+\varsigma_{2} a_{1,1} \mathbf{u}_{1}^{*}+\varsigma_{3} a_{2,1} \mathbf{u}_{2}^{*}\right\|^{2} \\
& =\varsigma_{1}{ }^{2}\left\|\alpha \mathbf{v}^{*}+\mathbf{z}\right\|^{2}+2 \varsigma_{1} \varsigma_{2}\left\langle\alpha \mathbf{v}^{*}+\mathbf{z}, a_{1,1} \mathbf{u}_{1}^{*}\right\rangle+\varsigma_{2}{ }^{2}\left\|a_{1,1} \mathbf{u}_{1}^{*}\right\|^{2} \\
& \quad+2 \varsigma_{1} \varsigma_{3}\left\langle\alpha \mathbf{v}^{*}+\mathbf{z}, a_{2,1} \mathbf{u}_{2}^{*}\right\rangle+2 \varsigma_{2} \varsigma_{3}\left\langle a_{1,1} \mathbf{u}_{1}^{*}, a_{2,1} \mathbf{u}_{2}^{*}\right\rangle+\varsigma_{3}{ }^{2}\left\|a_{2,1} \mathbf{u}_{2}^{*}\right\|^{2} \\
& =\varsigma_{1}{ }^{2}\left(\left\|\left[a_{1,2}+a_{2,2}\right] \mathbf{v}^{*}\right\|^{2}+2\left\langle\alpha \mathbf{v}^{*}, \mathbf{z}\right\rangle+\|\mathbf{z}\|^{2}\right)+2 \varsigma_{1} \varsigma_{2}\left(\left\langle\alpha \mathbf{v}^{*}, a_{1,1} \mathbf{u}_{1}^{*}\right\rangle+\left\langle\mathbf{z}, a_{1,1} \mathbf{u}_{1}^{*}\right\rangle\right) \\
& \quad+\varsigma_{2}{ }^{2}\left(n \bar{\beta}_{1} P_{1}\right)+2 \varsigma_{1} \varsigma_{3}\left[\left\langle\alpha \mathbf{v}^{*}, a_{2,1} \mathbf{u}_{2}^{*}\right\rangle+\left\langle\mathbf{z}, a_{2,1} \mathbf{u}_{2}^{*}\right\rangle\right]+2 \varsigma_{2} \varsigma_{3}\left\langle a_{1,1} \mathbf{u}_{1}^{*}, a_{2,1} \mathbf{u}_{2}^{*}\right\rangle+\varsigma_{3}{ }^{2}\left(n \bar{\beta}_{2} P_{2}\right) \\
& \stackrel{(a)}{\leq} n\left(\frac{1}{n} \varsigma_{1}{ }^{2}\left\|\alpha \mathbf{v}^{*}\right\|^{2}+\frac{2}{n} \varsigma_{1} \varsigma_{3}\left\|\alpha \mathbf{v}^{*}\right\| \sqrt{n \bar{\beta}_{2} P_{2}} \bar{\rho}+\varsigma_{1}{ }^{2} N+\varsigma_{2}{ }^{2} \bar{\beta}_{1} P_{1}\right. \\
& \left.\quad+2 \varsigma_{2} \varsigma_{3} \sqrt{\bar{\beta}_{1} \bar{\beta}_{2} P_{1} P_{2}} \tilde{\rho}+\varsigma_{3}{ }^{2} \bar{\beta}_{2} P_{2}+\kappa \epsilon\right),
\end{aligned}
$$

where in (a) we have used that $\left(\mathbf{s}_{1}, \mathbf{s}_{2}, \mathcal{C}_{1}, \mathcal{C}_{2}, \mathcal{C}_{c}, \mathbf{z}\right) \in \mathcal{E}_{\mathbf{X}}^{c}$, and Statement A) and Statement A1).

E) For every $\left(\mathbf{s}_{1}, \mathbf{s}_{2}, \mathcal{C}_{1}, \mathcal{C}_{2}, \mathcal{C}_{c}, \mathbf{z}\right) \in \mathcal{E}_{\mathbf{X}}^{c} \cap \mathcal{E}_{\mathbf{Z}}^{c}$ and an arbitrary $\mathbf{v} \in \mathcal{S}_{c}$,

$$
\left|\bar{\rho}-\cos \varangle\left(\mathbf{v}, \mathbf{u}_{2}^{*}\right)\right| \leq 7 \epsilon \text { and }\left|\cos \varangle\left(\mathbf{v}, \mathbf{u}_{1}^{*}\right)\right| \leq 7 \epsilon \text { and }
$$




$$
\begin{aligned}
& \left\|\mathbf{y}-\left(a_{1,1} \mathbf{u}_{1}^{*}+a_{2,1} \mathbf{u}_{2}^{*}+\alpha \mathbf{v}\right)\right\|^{2} \leq\left\|\mathbf{y}-\left(a_{1,1} \mathbf{u}_{1}^{*}+a_{2,1} \mathbf{u}_{2}^{*}+\alpha \mathbf{v}^{*}\right)\right\|^{2} \\
& \quad \Longrightarrow\|\alpha \mathbf{v}-\mathbf{w}\|^{2} \leq \Upsilon(\epsilon),
\end{aligned}
$$

where

$$
\begin{aligned}
\Upsilon(\epsilon)= & n \frac{\sigma^{2} \alpha^{2}\left(1-2^{-2 R_{\mathrm{c}}}\right) 2^{-2 R_{1}}\left\{1-\tilde{\rho}^{2}-\bar{\rho}^{2}\right\} N}{\left[\sigma^{2} \alpha^{2}\left(1-2^{-2 R_{\mathrm{c}}}\right) 2^{-2 R_{1}}\left[1-\tilde{\rho}^{2}-\bar{\rho}^{2}\right]+N\left[1-\tilde{\rho}^{2}\right]\right]} \\
& +n \alpha^{2} N^{2} \bar{\rho}^{2} \frac{\left(\bar{\rho}^{2} \bar{\beta}_{1} P_{1}-\sigma^{2} \tilde{\rho}^{2}\left(1-2^{-2 R_{\mathrm{c}}}\right) 2^{-2 R_{1}}\right)}{\left[\sigma^{2} \alpha^{2}\left(1-2^{-2 R_{\mathrm{c}}}\right) 2^{-2 R_{1}}\left[1-\tilde{\rho}^{2}-\bar{\rho}^{2}\right]+N\left[1-\tilde{\rho}^{2}\right]\right]^{2}}+n \kappa^{\prime} \epsilon,
\end{aligned}
$$

and where $\kappa^{\prime}$ depends only on $P_{2}, N_{1}, N_{2}, \varsigma_{1}, \varsigma_{2}$ and $\varsigma_{3}$.

Statement E) follows from combining Statement C) with Statement D) and the explicit values of $\varsigma_{1}, \varsigma_{2}$ and $\varsigma_{3}$ given in (139).

F) For every $\mathbf{v} \in \mathcal{S}_{c}$, denote by $\varphi \in[0, \pi]$ the angle between $\mathbf{v}$ and $\mathbf{w}$, and let

$$
\mathcal{B}\left(\mathbf{s}_{1}, \mathbf{s}_{2}, \mathbf{u}_{1}^{*}, \mathbf{v}^{*}, \mathbf{u}_{2}^{*}, \mathbf{z}\right) \triangleq\left\{\mathbf{v} \in S_{c}^{(n)}: \quad \cos (\varphi) \geq \sqrt{1-\frac{\Upsilon(\epsilon)}{n \alpha^{2} \sigma^{2} 2^{-2 R_{1}}\left(1-2^{-2 R_{\mathrm{c}}}\right)}}\right\},
$$

where $\epsilon$ is sufficiently large such that the term inside the square is non-negative.

Then, for every $\left(\mathbf{s}_{1}, \mathbf{s}_{2}, \mathcal{C}_{1}, \mathcal{C}_{2}, \mathcal{C}_{c}, \mathbf{z}\right) \in \mathcal{E}_{\mathbf{X}}^{c} \cap \mathcal{E}_{\mathbf{Z}}^{c}$,

$$
\begin{aligned}
& \left|\bar{\rho}-\cos \varangle\left(\mathbf{v}, \mathbf{u}_{2}^{*}\right)\right| \leq 7 \epsilon \quad \text { and } \quad\left|\cos \varangle\left(\mathbf{v}, \mathbf{u}_{1}^{*}\right)\right| \leq 7 \epsilon \quad \text { and } \\
& \left\|\mathbf{y}-\left(a_{1,1} \mathbf{u}_{1}^{*}+a_{2,1} \mathbf{u}_{2}^{*}+\alpha \mathbf{v}\right)\right\|^{2} \leq\left\|\mathbf{y}-\left(a_{1,1} \mathbf{u}_{1}^{*}+a_{2,1} \mathbf{u}_{2}^{*}+\left(a_{1,2}+a_{2,2}\right) \mathbf{v}^{*}\right)\right\|^{2} \\
& \quad \Longrightarrow \mathbf{v} \in \mathcal{B}\left(\mathbf{s}_{1}, \mathbf{s}_{2}, \mathbf{u}_{1}^{*}, \mathbf{v}^{*}, \mathbf{u}_{2}^{*}, \mathbf{z}\right) .
\end{aligned}
$$

Statement F) follows from Statement E) by noting that if $\mathbf{w} \neq 0$ and

$$
1-\frac{\Upsilon(\epsilon)}{n \alpha^{2} \sigma^{2} 2^{-2 R_{1}}\left(1-2^{-2 R_{c}}\right)}>0,
$$

then

$$
\begin{aligned}
& \left.\begin{array}{l}
\|\alpha \mathbf{v}\|^{2}=n \alpha^{2} \sigma^{2} 2^{-2 R_{1}}\left(1-2^{-2 R_{\mathrm{c}}}\right) \\
\|\alpha \mathbf{v}-\mathbf{w}\|^{2} \leq \Upsilon(\epsilon)
\end{array}\right\} \\
& \Longrightarrow \cos \varangle(\mathbf{u}, \mathbf{w}) \geq \sqrt{1-\frac{\Upsilon(\epsilon)}{n \alpha^{2} \sigma^{2} 2^{-2 R_{1}}\left(1-2^{-2 R_{\mathrm{c}}}\right)}},
\end{aligned}
$$

which follows by the same argument as (123).

The proof of Lemma 18 is now concluded by noticing that the set $\mathcal{E}_{\hat{\mathbf{v}}}^{\prime}$, defined in (140), is the set of tuples $\left(\mathbf{s}_{1}, \mathbf{s}_{2}, \mathcal{C}_{1}, \mathcal{C}_{2}, \mathcal{C}_{c}, \mathbf{z}\right)$ for which there exists a $\mathbf{v}(j) \in \mathcal{C}_{c} \backslash\left\{\mathbf{v}^{*}\right\}$ such that $\mathbf{v}(j) \in \mathcal{B}\left(\mathbf{s}_{1}, \mathbf{s}_{2}, \mathbf{u}_{1}^{*}, \mathbf{v}^{*}, \mathbf{u}_{2}^{*}, \mathbf{z}\right)$. Thus, by Statement F) and by the definition of $\mathcal{E}_{\hat{\mathbf{v}}}$ in 89 it follows that

$$
\mathcal{E}_{\hat{\mathbf{V}}} \cap \mathcal{E}_{\mathbf{S}}^{c} \cap \mathcal{E}_{\mathbf{X}}^{c} \cap \mathcal{E}_{\mathbf{Z}}^{c} \subseteq \mathcal{E}_{\hat{\mathbf{V}}}^{\prime} \cap \mathcal{E}_{\mathbf{S}}^{c} \cap \mathcal{E}_{\mathbf{X}}^{c} \cap \mathcal{E}_{\mathbf{Z}}^{c},
$$

and therefore

$$
\operatorname{Pr}\left[\mathcal{E}_{\hat{\mathbf{V}}} \cap \mathcal{E}_{\mathbf{S}}^{c} \cap \mathcal{E}_{\mathbf{X}}^{c} \cap \mathcal{E}_{\mathbf{Z}}^{c}\right] \leq \operatorname{Pr}\left[\mathcal{E}^{\prime} \hat{\mathbf{V}} \cap \mathcal{E}_{\mathbf{S}}^{c} \cap \mathcal{E}_{\mathbf{X}}^{c} \cap \mathcal{E}_{\mathbf{V}}^{c}\right]
$$

Next,

$$
\begin{aligned}
\operatorname{Pr}\left[\mathcal{E}_{\hat{\mathbf{V}}} \cap \mathcal{E}_{\mathbf{S}}^{c} \cap \mathcal{E}_{\mathbf{X}}^{c} \cap \mathcal{E}_{\mathbf{Z}}^{c}\right] \stackrel{(a)}{\stackrel{(a)}{\leq}} \operatorname{Pr}\left[\mathcal{E}_{\hat{\mathbf{v}}}^{\prime} \cap \mathcal{E}_{\mathbf{S}}^{c} \cap \mathcal{E}_{\mathbf{X}}^{c} \cap \mathcal{E}_{\mathbf{Z}}^{c}\right] \\
\stackrel{(b)}{\leq} \operatorname{Pr}\left[\mathcal{E}^{\prime} \hat{\mathbf{v}}^{\prime} \mid \mathcal{E}_{\mathbf{X}_{v}}^{c}\right]
\end{aligned}
$$


where (a) follows by Lemma 18 and (b) follows because $\mathcal{E}_{\mathbf{X}}^{c} \subseteq \mathcal{E}_{\mathbf{X}_{v}}^{c}$. The proof of 104) is now completed by combining (150) with Lemma 16. This gives that for every $\delta>0$ and every $\epsilon>0$ there exists some $n^{\prime}(\delta, \epsilon)$ such that for all $n>n^{\prime}(\delta, \epsilon)$, we have

$$
\operatorname{Pr}\left[\mathcal{E}_{\hat{\mathbf{V}}} \cap \mathcal{E}_{\mathbf{S}}^{c} \cap \mathcal{E}_{\mathbf{X}}^{c} \cap \mathcal{E}_{\mathbf{Z}}^{c}\right] \leq \operatorname{Pr}\left[\mathcal{E}_{\hat{\mathbf{V}}}^{\prime} \mid \mathcal{E}_{\mathbf{X}_{V}}^{c}\right]<\delta,
$$

whenever

$$
R_{\mathrm{c}}<\frac{1}{2} \log \left(\frac{\eta^{2}\left(1-\tilde{\rho}^{2}-\bar{\rho}^{2}\right)+N\left(1-\tilde{\rho}^{2}\right)}{N\left(1-\tilde{\rho}^{2}-\bar{\rho}^{2}\right)+\lambda_{c}}-\kappa_{3} \epsilon\right),
$$

where $\kappa_{3}$ is a positive constant determined by $P_{1}, P_{2}, N, \varsigma_{1}, \varsigma_{2}$ and $\varsigma_{3}$.

\section{Proof of rate constraint 105}

Define

$$
\mathbf{w}\left(\mathbf{s}_{1}, \mathbf{s}_{2}, \mathcal{C}_{1}, \mathcal{C}_{2}, \mathcal{C}_{c}, \mathbf{z}\right)=\varsigma_{1}\left(\mathbf{y}-\alpha \mathbf{v}^{*}\right)+\varsigma_{2} \alpha \mathbf{v}^{*}
$$

where

$$
\begin{aligned}
& \varsigma_{1}=\frac{a_{1,1}^{2}\left(1-2^{-2 R_{1}}\right)+2 a_{1,1} a_{2,1} \rho\left(1-2^{-2 R_{1}}\right)\left(1-2^{-2 R_{2}}\right)+a_{2,1}^{2}\left(1-2^{-2 R_{2}}\right)\left(1-\bar{\rho}^{2}\right)}{a_{1,1}^{2}\left(1-2^{-2 R_{1}}\right)+2 a_{1,1} a_{2,1} \rho\left(1-2^{-2 R_{1}}\right)\left(1-2^{-2 R_{2}}\right)+a_{2,1}^{2}\left(1-2^{-2 R_{2}}\right)\left(1-\bar{\rho}^{2}\right)+\frac{N}{\sigma^{2}}} \\
& \varsigma_{2}=\frac{\frac{N}{\sigma^{2}} a_{2,1} \rho\left(1-2^{-2 R_{2}}\right)}{\alpha\left(a_{1,1}^{2}\left(1-2^{-2 R_{1}}\right)+2 a_{1,1} a_{2,1} \rho\left(1-2^{-2 R_{1}}\right)\left(1-2^{-2 R_{2}}\right)+a_{2,1}^{2}\left(1-2^{-2 R_{2}}\right)\left(1-\bar{\rho}^{2}\right)+\frac{N}{\sigma^{2}}\right)} .
\end{aligned}
$$

In the remainder we shall use the shorthand notation $\mathbf{w}$ instead of $\mathbf{w}\left(\mathbf{s}_{1}, \mathbf{s}_{2}, \mathcal{C}_{1}, \mathcal{C}_{2}, \mathcal{C}_{c}, \mathbf{z}\right)$. We now start with a lemma that will be used to prove 105.

Lemma 19 Let $\varphi_{j, l} \in[0, \pi]$ be the angle between $\mathbf{w}$ and $a_{1,1} \mathbf{u}_{1}(j)+a_{2,1} \mathbf{u}_{2}(l)$, and let the set $\mathcal{E}_{\left(\hat{\mathbf{U}}_{1}, \hat{\mathbf{U}}_{2}\right)}^{\prime}$ be defined as

$$
\begin{gathered}
\mathcal{E}_{\left(\hat{\mathbf{U}}_{1}, \hat{\mathbf{U}}_{2}\right)}^{\prime} \triangleq\left\{\left(\mathbf{s}_{1}, \mathbf{s}_{2}, \mathcal{C}_{1}, \mathcal{C}_{2}, \mathcal{C}_{c}, \mathbf{z}\right): \exists \mathbf{u}_{1}(j) \in \mathcal{C}_{1} \backslash\left\{\mathbf{u}_{1}^{*}\right\} \text { and } \exists \mathbf{u}_{2}(j) \in \mathcal{C}_{2} \backslash\left\{\mathbf{u}_{2}^{*}\right\}\right. \\
\text { s.t. } \left.\cos \left(\varphi_{j, l}\right) \geq \sqrt{1-\tilde{\Upsilon}-\kappa^{\prime \prime} \epsilon}\right\}
\end{gathered}
$$

where

$$
\tilde{\Upsilon} \triangleq \frac{\left(\bar{\beta}_{1} P_{1}+2 \sqrt{\bar{\beta}_{1} \bar{\beta}_{2} P_{1} P_{2}} \tilde{\rho}+\bar{\beta}_{2} P_{2}\left(1-\bar{\rho}^{2}\right)\right) N}{\left(\bar{\beta}_{1} P_{1}+2 \sqrt{\bar{\beta}_{1} P_{1} \bar{\beta}_{2} P_{2}} \tilde{\rho}+\bar{\beta}_{2} P_{2}\left[1-\bar{\rho}^{2}\right]+N\right)\left(\bar{\beta}_{1} P_{1}+2 \tilde{\rho} \sqrt{\bar{\beta}_{1} P_{1} \bar{\beta}_{2} P_{2}}+\bar{\beta}_{2} P_{2}\right)},
$$

and $\kappa^{\prime \prime}$ is a positive constant determined by $P_{1}, P_{2}, N$, $\varsigma_{1}$ and $\varsigma_{2}$. Then,

$$
\mathcal{E}_{\left(\hat{\mathbf{U}}_{1}, \hat{\mathbf{U}}_{2}\right)} \cap \mathcal{E}_{\mathbf{S}}^{c} \cap \mathcal{E}_{\mathbf{X}}^{c} \cap \mathcal{E}_{\mathbf{Z}}^{c} \subseteq \mathcal{E}_{\left(\hat{\mathbf{U}}_{1}, \hat{\mathbf{U}}_{2}\right)}^{\prime} \cap \mathcal{E}_{\mathbf{S}}^{c} \cap \mathcal{E}_{\mathbf{X}}^{c} \cap \mathcal{E}_{\mathbf{Z}}^{c}
$$

and, in particular

$$
\operatorname{Pr}\left[\mathcal{E}_{\left(\hat{\mathbf{U}}_{1}, \hat{\mathbf{U}}_{2}\right)} \cap \mathcal{E}_{\mathbf{S}}^{c} \cap \mathcal{E}_{\mathbf{X}}^{c} \cap \mathcal{E}_{\mathbf{Z}}^{c}\right] \leq \operatorname{Pr}\left[\mathcal{E}_{\left(\hat{\mathbf{U}}_{1}, \hat{\mathbf{U}}_{2}\right)}^{\prime} \cap \mathcal{E}_{\mathbf{S}}^{c} \cap \mathcal{E}_{\mathbf{X}}^{c} \cap \mathcal{E}_{\mathbf{Z}}^{c}\right] .
$$

Proof: We first recall that, for the event $\mathcal{E}_{\left(\hat{\mathbf{U}}_{1}, \hat{\mathbf{U}}_{2}\right)}$ to occur, there must exist codewords 
$\mathbf{u}_{1}(j) \in \mathcal{C}_{1} \backslash\left\{\mathbf{u}_{1}^{*}\right\}$ and $\mathbf{u}_{2}(l) \in \mathcal{C}_{2} \backslash\left\{\mathbf{u}_{2}^{*}\right\}$ that satisfy the following four conditions

$$
\begin{aligned}
\left|\tilde{\rho}-\cos \varangle\left(\mathbf{u}_{1}(j), \mathbf{u}_{2}(l)\right)\right| & \leq 7 \epsilon \\
\left|\bar{\rho}-\cos \varangle\left(\mathbf{v}^{*}, \mathbf{u}_{2}(l)\right)\right| & \leq 7 \epsilon \\
\left|\cos \varangle\left(\mathbf{v}^{*}, \mathbf{u}_{1}(j)\right)\right| & \leq 3 \epsilon \\
\left\|\mathbf{y}-\mathbf{X}_{\mathbf{u}_{1}(j), \mathbf{v}^{*}, \mathbf{u}_{2}(l)}\right\|^{2} & \leq\left\|\mathbf{y}-\mathbf{X}_{\mathbf{u}_{1}^{*}, \mathbf{v}^{*}, \mathbf{u}_{2}^{*}}\right\|^{2} .
\end{aligned}
$$

The proof is now based on a sequence of statements related to these conditions:

A) For every $\left(\mathbf{s}_{1}, \mathbf{s}_{2}, \mathcal{C}_{1}, \mathcal{C}_{2}, \mathcal{C}_{c}, \mathbf{z}\right) \in \mathcal{E}_{\mathbf{X}}^{c}$ and every $\mathbf{u}_{1} \in \mathcal{S}_{1}$ and $\mathbf{u}_{2} \in \mathcal{S}_{2}$,

$$
\left|\tilde{\rho}-\cos \varangle\left(\mathbf{u}_{1}, \mathbf{u}_{2}\right)\right| \leq 7 \epsilon \Longrightarrow\left|n \tilde{\rho} \sqrt{\bar{\beta}_{1} \bar{\beta}_{2} P_{1} P_{2}}-\left\langle a_{1,1} \mathbf{u}_{1}, a_{2,1} \mathbf{u}_{2}\right\rangle\right| \leq 7 n \sqrt{\bar{\beta}_{1} \bar{\beta}_{2} P_{1} P_{2}} \epsilon .
$$

Statement A) follows by rewriting $\cos \varangle\left(\mathbf{u}_{1}, \mathbf{u}_{2}\right)$ as $\left\langle\mathbf{u}_{1}, \mathbf{u}_{2}\right\rangle /\left(\left\|\mathbf{u}_{1}\right\|\left\|\mathbf{u}_{2}\right\|\right)$, and then multiplying the inequality on the 1.h.s. of (157) by $\left\|a_{1,1} \mathbf{u}_{1}\right\| \cdot\left\|a_{2,1} \mathbf{u}_{2}\right\|$ and recalling that $\left\|a_{1,1} \mathbf{u}_{1}\right\|=$ $\sqrt{n \bar{\beta}_{1} P_{1}}$ and that $\left\|a_{2,1} \mathbf{u}_{2}\right\|=\sqrt{n \bar{\beta}_{2} P_{2}}$.

A1) For every $\left(\mathbf{s}_{1}, \mathbf{s}_{2}, \mathcal{C}_{1}, \mathcal{C}_{2}, \mathcal{C}_{c}, \mathbf{z}\right) \in \mathcal{E}_{\mathbf{X}}^{c}$,

$$
\begin{aligned}
& \left|\cos \varangle\left(\mathbf{v}^{*}, \mathbf{u}_{1}\right)\right| \leq 3 \epsilon \\
& \Longrightarrow\left|\left\langle\alpha \mathbf{v}^{*}, a_{1,1} \mathbf{u}_{1}\right\rangle\right| \leq 3\left\|\alpha \mathbf{v}^{*}\right\| \sqrt{n \bar{\beta}_{1} P_{1}} \epsilon .
\end{aligned}
$$

Statement A1) follows by rewriting $\cos \varangle\left(\mathbf{v}^{*}, \mathbf{u}_{1}^{*}\right)$ as $\left\langle\mathbf{v}^{*}, \mathbf{u}_{1}\right\rangle /\left(\left\|\mathbf{v}^{*}\right\|\left\|\mathbf{u}_{1}\right\|\right)$, and then multiplying the inequality on the 1.h.s. of (158) by $\left\|\alpha \mathbf{v}^{*}\right\| \cdot\left\|a_{1,1} \mathbf{u}_{1}\right\|$.

A2) For every $\left(\mathbf{s}_{1}, \mathbf{s}_{2}, \mathcal{C}_{1}, \mathcal{C}_{2}, \mathcal{C}_{c}, \mathbf{z}\right) \in \mathcal{E}_{\mathbf{X}}^{c}$ and every $\mathbf{u}_{2} \in \mathcal{S}_{2}$,

$$
\begin{aligned}
& \left|\bar{\rho}-\cos \varangle\left(\mathbf{v}^{*}, \mathbf{u}_{2}\right)\right| \leq 7 \epsilon \\
& \quad \Longrightarrow\left|\left\|\alpha \mathbf{v}^{*}\right\| \sqrt{n \bar{\beta}_{2} P_{2}} \bar{\rho}-\left\langle\alpha \mathbf{v}^{*}, a_{2,1} \mathbf{u}_{2}\right\rangle\right| \leq 7 \epsilon\left\|\alpha \mathbf{v}^{*}\right\| \sqrt{n \bar{\beta}_{2} P_{2}} .
\end{aligned}
$$

Statement A2) follows by rewriting $\cos \varangle\left(\mathbf{v}^{*}, \mathbf{u}_{2}\right)$ as $\left\langle\mathbf{v}^{*}, \mathbf{u}_{2}\right\rangle /\left(\left\|\mathbf{v}^{*}\right\|\left\|\mathbf{u}_{2}\right\|\right)$, and then multiplying the inequality on the 1.h.s. of (159) by $\left\|\alpha \mathbf{v}^{*}\right\| \cdot\left\|a_{2,1} \mathbf{u}_{2}\right\|$.

B) For every $\left(\mathbf{s}_{1}, \mathbf{s}_{2}, \mathcal{C}_{1}, \mathcal{C}_{2}, \mathcal{C}_{c}, \mathbf{z}\right) \in \mathcal{E}_{\mathbf{X}}^{c} \cap \mathcal{E}_{\mathbf{Z}}^{c}$ and every $\mathbf{u}_{1} \in \mathcal{S}_{1}$ and $\mathbf{u}_{2} \in \mathcal{S}_{2}$,

$$
\begin{aligned}
& \mid \mathbf{y}-\mathbf{X}_{\mathbf{u}_{1}, \mathbf{v}^{*}, \mathbf{u}_{2}}\left\|^{2} \leq\right\| \mathbf{y}-\mathbf{X}_{\mathbf{u}_{1}^{*}, \mathbf{v}^{*}, \mathbf{u}_{2}^{*}} \|^{2} \\
& \quad \Longrightarrow\left\langle\mathbf{y}-\alpha \mathbf{v}^{*}, a_{1,1} \mathbf{u}_{1}+a_{2,1} \mathbf{u}_{2}\right\rangle \geq n\left(\bar{\beta}_{1} P_{1}+2 \sqrt{\bar{\beta}_{1} \bar{\beta}_{2} P_{1} P_{2}}(\widetilde{\rho}-7 \epsilon)+\bar{\beta}_{2} P_{2}-\kappa \epsilon\right) .
\end{aligned}
$$

Statement B) follows from rewriting the inequality on the 1.h.s. of (160) as

$$
\left\|\left(\mathbf{y}-\alpha \mathbf{v}^{*}\right)-\left(a_{1,1} \mathbf{u}_{1}+a_{2,1} \mathbf{u}_{2}\right)\right\|^{2} \leq\left\|\left(\mathbf{y}-\alpha \mathbf{v}^{*}\right)-\left(a_{1,1} \mathbf{u}_{1}^{*}+a_{2,1} \mathbf{u}_{2}^{*}\right)\right\|^{2},
$$

or equivalently as

$$
\begin{aligned}
\left\langle\left(\mathbf{y}-\alpha \mathbf{v}^{*}\right), a_{1,1} \mathbf{u}_{1}+a_{2,1} \mathbf{u}_{2}\right\rangle & \geq\left\langle\left(\mathbf{y}-\alpha \mathbf{v}^{*}\right), a_{1,1} \mathbf{u}_{1}^{*}+a_{2,1} \mathbf{u}_{2}^{*}\right\rangle \\
& =\left\langle a_{1,1} \mathbf{u}_{1}^{*}+a_{2,1} \mathbf{u}_{2}^{*}+\mathbf{z}, a_{1,1} \mathbf{u}_{1}^{*}+a_{2,1} \mathbf{u}_{2}^{*}\right\rangle \\
& =\left\|a_{1,1} \mathbf{u}_{1}^{*}+a_{2,1} \mathbf{u}_{2}^{*}\right\|^{2}+\left\langle\mathbf{z}, a_{1,1} \mathbf{u}_{1}^{*}+a_{2,1} \mathbf{u}_{2}^{*}\right\rangle .
\end{aligned}
$$

It now follows from the equivalence of the first inequality in (160) with (161) that for $\left(\mathbf{s}_{1}, \mathbf{s}_{2}, \mathcal{C}_{1}, \mathcal{C}_{2}, \mathcal{C}_{c}, \mathbf{z}\right) \in \mathcal{E}_{\mathbf{Z}}^{c}$, the first inequality in 160$)$ can only hold if

$$
\left\langle\mathbf{y}-\alpha \mathbf{v}^{*}, a_{1,1} \mathbf{u}_{1}+a_{2,1} \mathbf{u}_{2}\right\rangle \geq n\left(\bar{\beta}_{1} P_{1}+2 \sqrt{\bar{\beta}_{1} \bar{\beta}_{2} P_{1} P_{2}}(\widetilde{\rho}-7 \epsilon)+\bar{\beta}_{2} P_{2}-\kappa \epsilon\right),
$$


thus establishing B).

C) For every $\left(\mathbf{s}_{1}, \mathbf{s}_{2}, \mathcal{C}_{1}, \mathcal{C}_{2}, \mathcal{C}_{c}, \mathbf{z}\right) \in \mathcal{E}_{\mathbf{X}}^{c} \cap \mathcal{E}_{\mathbf{Z}}^{c}$ and every $\mathbf{u}_{1} \in \mathcal{S}_{1}$ and $\mathbf{u}_{2} \in \mathcal{S}_{2}$,

$\left|\tilde{\rho}-\cos \varangle\left(\mathbf{u}_{1}, \mathbf{u}_{2}\right)\right| \leq 7 \epsilon$ and $\left|\bar{\rho}-\cos \varangle\left(\mathbf{u}_{2}, \mathbf{v}^{*}\right)\right| \leq 7 \epsilon$ and $\left|\cos \varangle\left(\mathbf{u}_{1}, \mathbf{v}^{*}\right)\right| \leq 7 \epsilon$ and $\mid \mathbf{y}-\mathbf{X}_{\mathbf{u}_{1}, \mathbf{v}^{*}, \mathbf{u}_{2}}\left\|^{2} \leq\right\| \mathbf{y}-\mathbf{X}_{\mathbf{u}_{1}^{*}, \mathbf{v}^{*}, \mathbf{u}_{2}^{*}} \|^{2}$

$$
\begin{aligned}
\left\|a_{1,1} \mathbf{u}_{1}+a_{2,1} \mathbf{u}_{2}-\mathbf{w}\right\|^{2} \leq & n\left(\left(\bar{\beta}_{1} P_{1}+2 \sqrt{\bar{\beta}_{1} \bar{\beta}_{2} P_{1} P_{2}} \tilde{\rho}+\bar{\beta}_{2} P_{2}\right)\left(1-2 \varsigma_{1}\right)\right. \\
& \left.-2 \varsigma_{2} \frac{1}{n}\left\|\alpha v^{*}\right\| \sqrt{n \bar{\beta}_{2} P_{2}} \bar{\rho}\right)+\|\mathbf{w}\|^{2}+n k^{\prime} \epsilon .
\end{aligned}
$$

Statement C) is obtained as follows:

$$
\begin{aligned}
& \left\|a_{1,1} \mathbf{u}_{1}+a_{2,1} \mathbf{u}_{2}-\mathbf{w}\right\|^{2}=\left\|a_{1,1} \mathbf{u}_{1}+a_{2,1} \mathbf{u}_{2}\right\|^{2}-2\left\langle a_{1,1} \mathbf{u}_{1}+a_{2,1} \mathbf{u}_{2}, \mathbf{w}\right\rangle+\|\mathbf{w}\|^{2} \\
& =\left\|a_{1,1} \mathbf{u}_{1}+a_{2,1} \mathbf{u}_{2}\right\|^{2}-2\left\langle a_{1,1} \mathbf{u}_{1}+a_{2,1} \mathbf{u}_{2}, \varsigma_{1}\left(a_{1,1} \mathbf{u}_{1}^{*}+a_{2,1} \mathbf{u}_{2}^{*}+\mathbf{z}\right)+\varsigma_{2} \alpha \mathbf{v}^{*}\right\rangle+\|\mathbf{w}\|^{2} \\
& \quad \stackrel{(a)}{\leq} n\left(\left(\bar{\beta}_{1} P_{1}+2 \sqrt{\bar{\beta}_{1} \bar{\beta}_{2} P_{1} P_{2}} \tilde{\rho}+\bar{\beta}_{2} P_{2}\right)\left(1-2 \varsigma_{1}\right)-2 \varsigma_{2} \frac{1}{n}\left\|\alpha v^{*}\right\| \sqrt{n \bar{\beta}_{2} P_{2}} \bar{\rho}\right)+\|\mathbf{w}\|^{2} \\
& \quad+n \kappa^{\prime} \epsilon,
\end{aligned}
$$

where in (a) we have used Statement A), A1), A2) and Statement B).

D) For every $\left(\mathbf{s}_{1}, \mathbf{s}_{2}, \mathcal{C}_{1}, \mathcal{C}_{2}, \mathcal{C}_{c}, \mathbf{z}\right) \in \mathcal{E}_{\mathbf{X}}^{c} \cap \mathcal{E}_{\mathbf{Z}}^{c}$

$$
\begin{aligned}
\|\mathbf{w}\|^{2} \leq & n\left(\varsigma_{1}^{2}\left(\bar{\beta}_{1} P_{1}+2 \sqrt{\bar{\beta}_{1} \bar{\beta}_{2} P_{1} P_{2}} \tilde{\rho}+\bar{\beta}_{2} P_{2}+N\right)+\frac{1}{n} 2 \varsigma_{1} \varsigma_{2} \alpha\left\|\mathbf{v}^{*}\right\| \sqrt{n \bar{\beta}_{2} P_{2}} \bar{\rho}\right. \\
& \left.+\frac{1}{n} \varsigma_{2}^{2} \alpha^{2}\left\|\mathbf{v}^{*}\right\|^{2}+k \epsilon\right)
\end{aligned}
$$

where $k$ depends on $P_{1}, P_{2}, N, \varsigma_{1}$ and $\varsigma_{2}$ only.

Statement D) is obtained as follows:

$$
\begin{aligned}
\|\mathbf{w}\|^{2}= & \left\|\varsigma_{1}\left(a_{1,1} \mathbf{u}_{1}^{*}+a_{2,1} \mathbf{u}_{2}^{*}+\mathbf{z}\right)+\varsigma_{2} \alpha \mathbf{v}^{*}\right\|^{2} \\
= & \varsigma_{1}{ }^{2}\left\|a_{1,1} \mathbf{u}_{1}^{*}+a_{2,1} \mathbf{u}_{2}^{*}+\mathbf{z}\right\|^{2}+2 \varsigma_{1} \varsigma_{2}\left\langle a_{1,1} \mathbf{u}_{1}^{*}+a_{2,1} \mathbf{u}_{2}^{*}+\mathbf{z}, \alpha \mathbf{v}^{*}\right\rangle+\varsigma_{2}{ }^{2}\left\|\alpha \mathbf{v}^{*}\right\|^{2} \\
= & \varsigma_{1}{ }^{2}\left(\left\|a_{1,1} \mathbf{u}_{1}^{*}+a_{2,1} \mathbf{u}_{2}^{*}\right\|^{2}+2\left\langle a_{1,1} \mathbf{u}_{1}^{*}+a_{2,1} \mathbf{u}_{2}^{*}, \mathbf{z}\right\rangle+\|\mathbf{z}\|^{2}\right) \\
& +2 \varsigma_{1} \varsigma_{2}\left\langle a_{1,1} \mathbf{u}_{1}^{*}+a_{2,1} \mathbf{u}_{2}^{*}+\mathbf{z}, \alpha \mathbf{v}^{*}\right\rangle+\varsigma_{2}{ }^{2} \alpha^{2}\left\|\mathbf{v}^{*}\right\|^{2} \\
& \stackrel{(a)}{\leq} n\left(\varsigma_{1}{ }^{2}\left(\bar{\beta}_{1} P_{1}+2 \sqrt{\bar{\beta}_{1} \bar{\beta}_{2} P_{1} P_{2}} \tilde{\rho}+\bar{\beta}_{2} P_{2}+N\right)+\frac{1}{n} 2 \varsigma_{1} \varsigma_{2} \alpha\left\|\mathbf{v}^{*}\right\| \sqrt{n \bar{\beta}_{2} P_{2}} \bar{\rho}\right. \\
& \left.+\frac{1}{n} \varsigma_{2}{ }^{2} \alpha^{2}\left\|\mathbf{v}^{*}\right\|^{2}+\kappa \epsilon\right),
\end{aligned}
$$

where in (a) we have used that $\left(\mathbf{s}_{1}, \mathbf{s}_{2}, \mathcal{C}_{1}, \mathcal{C}_{2}, \mathcal{C}_{c}, \mathbf{z}\right) \in \mathcal{E}_{\mathbf{X}}^{c}$, and statements A), A1) and A2).

E) For every $\left(\mathbf{s}_{1}, \mathbf{s}_{2}, \mathcal{C}_{1}, \mathcal{C}_{2}, \mathcal{C}_{c}, \mathbf{z}\right) \in \mathcal{E}_{\mathbf{X}}^{c} \cap \mathcal{E}_{\mathbf{Z}}^{c}$ and an arbitrary $\mathbf{u}_{1} \in \mathcal{S}_{1}$ and $\mathbf{u}_{2} \in \mathcal{S}_{2}$,

$\left|\tilde{\rho}-\cos \varangle\left(\mathbf{u}_{1}, \mathbf{u}_{2}\right)\right| \leq 7 \epsilon$ and $\left|\bar{\rho}-\cos \varangle\left(\mathbf{v}, \mathbf{u}_{2}\right)\right| \leq 7 \epsilon$ and $\mid \mathbf{y}-\mathbf{X}_{\mathbf{u}_{1}, \mathbf{v}^{*}, \mathbf{u}_{2}}\left\|^{2} \leq\right\| \mathbf{y}-\mathbf{X}_{\mathbf{u}_{1}^{*}, \mathbf{v}^{*}, \mathbf{u}_{2}^{*}} \|^{2}$

$\Longrightarrow\left\|a_{1,1} \mathbf{u}_{1}+a_{2,1} \mathbf{u}_{2}-\mathbf{w}\right\|^{2} \leq \Upsilon(\epsilon)$, 
where

$$
\Upsilon(\epsilon)=n \frac{\left(\bar{\beta}_{1} P_{1}+2 \sqrt{\bar{\beta}_{1} \bar{\beta}_{2} P_{1} P_{2}} \tilde{\rho}+\bar{\beta}_{2} P_{2}\left(1-\bar{\rho}^{2}\right)\right) N}{\bar{\beta}_{1} P_{1}+2 \sqrt{\bar{\beta}_{1} \bar{\beta}_{2} P_{1} P_{2}} \tilde{\rho}+\bar{\beta}_{2} P_{2}\left(1-\bar{\rho}^{2}\right)+N}+n k^{\prime} \epsilon,
$$

and where $k^{\prime}$ only depends on $P_{1}, N_{1}, N_{2}, \varsigma_{1}$ and $\varsigma_{2}$.

Statement E) follows from combining Statement C) with Statement D) and the explicit values of $\varsigma_{1}$ and $\varsigma_{2}$ given in (151).

F) For every $\mathbf{u}_{1} \in \mathcal{S}_{1}, \mathbf{u}_{2} \in \mathcal{S}_{2}$, denote by $\varphi \in[0, \pi]$ the angle between $a_{1,1} \mathbf{u}_{1}+a_{2,1} \mathbf{u}_{2}$ and $\mathbf{w}$, and let

$$
\begin{aligned}
\mathcal{B}\left(\mathbf{s}_{1}, \mathbf{s}_{2}, \mathbf{u}_{1}^{*}, \mathbf{v}^{*}, \mathbf{u}_{2}^{*}, \mathbf{z}\right) \triangleq\left\{\mathbf{u}_{1} \in S_{1}^{(n)}, \mathbf{u}_{2} \in S_{2}^{(n)}:\right. & \\
& \left.\quad \cos (\varphi) \geq \sqrt{1-\frac{\Upsilon(\epsilon)}{n\left(\bar{\beta}_{1} P_{1}+2 \tilde{\rho} \sqrt{\bar{\beta}_{1} P_{1} \bar{\beta}_{2} P_{2}}+\bar{\beta}_{2} P_{2}\right)}}\right\},
\end{aligned}
$$

where $\epsilon$ is sufficiently small such that the term inside the square is non-negative. Then, for every $\left(\mathbf{s}_{1}, \mathbf{s}_{2}, \mathcal{C}_{1}, \mathcal{C}_{2}, \mathcal{C}_{c}, \mathbf{z}\right) \in \mathcal{E}_{\mathbf{X}}^{c} \cap \mathcal{E}_{\mathbf{Z}}^{c}$,

$$
\begin{aligned}
& \left|\tilde{\rho}-\cos \varangle\left(\mathbf{u}_{1}, \mathbf{u}_{2}\right)\right| \leq 7 \epsilon \quad \text { and } \quad\left|\bar{\rho}-\cos \varangle\left(\mathbf{v}^{*}, \mathbf{u}_{2}\right)\right| \leq 7 \epsilon \quad \text { and } \\
& \left|\cos \varangle\left(\mathbf{v}^{*}, \mathbf{u}_{1}\right)\right| \leq 3 \epsilon \quad \text { and } \quad \mid \mathbf{y}-\mathbf{X}_{\mathbf{u}_{1}, \mathbf{v}^{*}, \mathbf{u}_{2}}\left\|^{2} \leq\right\| \mathbf{y}-\mathbf{X}_{\mathbf{u}_{1}^{*}, \mathbf{v}^{*}, \mathbf{u}_{2}^{*}} \|^{2} \\
& \Longrightarrow \quad a_{1,1} \mathbf{u}_{1}+a_{2,1} \mathbf{u}_{2} \in \mathcal{B}\left(\mathbf{s}_{1}, \mathbf{s}_{2}, \mathbf{u}_{1}^{*}, \mathbf{v}^{*}, \mathbf{u}_{2}^{*}, \mathbf{z}\right) .
\end{aligned}
$$

Statement $\mathrm{F}$ ) follows from Statement $\mathrm{E}$ ) by noting that if $\mathbf{w} \neq 0$ and

$$
1-\frac{\Upsilon(\epsilon)}{n\left(\bar{\beta}_{1} P_{1}+2 \tilde{\rho} \sqrt{\bar{\beta}_{1} P_{1} \bar{\beta}_{2} P_{2}}+\bar{\beta}_{2} P_{2}\right)}>0,
$$

then

$$
\begin{aligned}
& \left\|a_{1,1} \mathbf{u}_{1}+a_{2,1} \mathbf{u}_{2}\right\|^{2}=n\left(\bar{\beta}_{1} P_{1}+2 \tilde{\rho} \sqrt{\bar{\beta}_{1} P_{1} \bar{\beta}_{2} P_{2}}+\bar{\beta}_{2} P_{2}\right) \\
& \text { and }\left\|a_{1,1} \mathbf{u}_{1}+a_{2,1} \mathbf{u}_{2}-\mathbf{w}\right\|^{2} \leq \Upsilon(\epsilon) \\
& \quad \Longrightarrow \cos \varangle\left(a_{1,1} \mathbf{u}_{1}+a_{2,1} \mathbf{u}_{2}, \mathbf{w}\right) \geq \sqrt{1-\frac{\Upsilon(\epsilon)}{n\left(\bar{\beta}_{1} P_{1}+2 \tilde{\rho} \sqrt{\bar{\beta}_{1} P_{1} \bar{\beta}_{2} P_{2}}+\bar{\beta}_{2} P_{2}\right)}} .
\end{aligned}
$$

To see this, first note that every $a_{1,1} \mathbf{u}_{1}+a_{2,1} \mathbf{u}_{2}$, where $\mathbf{u}_{1} \in \mathcal{S}_{1}, \mathbf{u}_{2} \in \mathcal{S}_{2}$, satisfying the condition on the 1.h.s. of (162) lies within a sphere of radius $\sqrt{\Upsilon(\epsilon)}$ centered at $\mathbf{w}$. In addition, for every $\mathbf{u}_{1} \in \mathcal{S}_{1}, \mathbf{u}_{2} \in \mathcal{S}_{2}$ we have that $a_{1,1} \mathbf{u}_{1}+a_{2,1} \mathbf{u}_{2}$ also lies on the centered $\mathbb{R}^{n}$-sphere of radius $n\left(\bar{\beta}_{1} P_{1}+2 \tilde{\rho} \sqrt{\bar{\beta}_{1} P_{1} \bar{\beta}_{2} P_{2}}+\bar{\beta}_{2} P_{2}\right)$. Hence, every $\mathbf{u}_{1} \in \mathcal{S}_{1}, \mathbf{u}_{2} \in \mathcal{S}_{2}$ satisfying the condition on the 1.h.s. of (162) lies in the intersection of these two regions, which is a polar cap on the centered sphere of radius $n\left(\bar{\beta}_{1} P_{1}+2 \tilde{\rho} \sqrt{\bar{\beta}_{1} P_{1} \bar{\beta}_{2} P_{2}}+\bar{\beta}_{2} P_{2}\right)$. Hence, every $\mathbf{u}_{1} \in \mathcal{S}_{1}^{(n)}, \mathbf{u}_{2} \in$ $\mathcal{S}_{2}^{(n)}$ satisfying the upper conditions of 162, also satisfies

$$
\begin{aligned}
\cos \varphi & \geq \sqrt{1-\frac{\Upsilon(\epsilon)}{n\left(\bar{\beta}_{1} P_{1}+2 \tilde{\rho} \sqrt{\bar{\beta}_{1} P_{1} \bar{\beta}_{2} P_{2}}+\bar{\beta}_{2} P_{2}\right)}} \\
& =\sqrt{1-\tilde{\Upsilon}-\kappa^{\prime \prime} \epsilon},
\end{aligned}
$$


where

$$
\begin{aligned}
& \tilde{\Upsilon} \triangleq \frac{\left(\bar{\beta}_{1} P_{1}+2 \sqrt{\bar{\beta}_{1} \bar{\beta}_{2} P_{1} P_{2}} \tilde{\rho}+\bar{\beta}_{2} P_{2}\left(1-\bar{\rho}^{2}\right)\right) N}{\left(\bar{\beta}_{1} P_{1}+2 \sqrt{\bar{\beta}_{1} P_{1} \bar{\beta}_{2} P_{2}} \tilde{\rho}+\bar{\beta}_{2} P_{2}\left(1-\bar{\rho}^{2}\right)+N\right)\left(\bar{\beta}_{1} P_{1}+2 \tilde{\rho} \sqrt{\bar{\beta}_{1} P_{1} \bar{\beta}_{2} P_{2}}+\bar{\beta}_{2} P_{2}\right)} \\
& \kappa^{\prime \prime} \triangleq \frac{k^{\prime}}{n\left(\bar{\beta}_{1} P_{1}+2 \tilde{\rho} \sqrt{\bar{\beta}_{1} P_{1} \bar{\beta}_{2} P_{2}}+\bar{\beta}_{2} P_{2}\right)} .
\end{aligned}
$$

The proof of Lemma 19 is now concluded by noticing that the set $\mathcal{E}_{\left(\hat{\mathbf{U}}_{1}, \hat{\mathbf{U}}_{2}\right)}$, defined in (152), is the set of tuples $\left(\mathbf{s}_{1}, \mathbf{s}_{2}, \mathcal{C}_{1}, \mathcal{C}_{2}, \mathcal{C}_{c}, \mathbf{z}\right)$ for which there exists a $\mathbf{u}_{1}(j) \in \mathcal{C}_{1} \backslash\left\{\mathbf{u}_{1}^{*}\right\}$ and $\mathbf{u}_{2}(l) \in \mathcal{C}_{2} \backslash\left\{\mathbf{u}_{2}^{*}\right\}$ such that $a_{1,1} \mathbf{u}_{1}(j)+a_{2,1} \mathbf{u}_{2}(l) \in \mathcal{B}\left(\mathbf{s}_{1}, \mathbf{s}_{2}, \mathbf{u}_{1}^{*}, \mathbf{v}^{*}, \mathbf{u}_{2}^{*}, \mathbf{z}\right)$. Thus, by Statement F) and by the definition of $\mathcal{E}_{\left(\hat{\mathbf{U}}_{1}, \hat{\mathbf{U}}_{2}\right)}$ in (90) it follows that

$$
\mathcal{E}_{\left(\hat{\mathbf{U}}_{1}, \hat{\mathbf{U}}_{2}\right)} \cap \mathcal{E}_{\mathbf{S}}^{c} \cap \mathcal{E}_{\mathbf{X}}^{c} \cap \mathcal{E}_{\mathbf{Z}}^{c} \subseteq \mathcal{E}_{\left(\hat{\mathbf{U}}_{1}, \hat{\mathbf{U}}_{2}\right)}^{\prime} \cap \mathcal{E}_{\mathbf{S}}^{c} \cap \mathcal{E}_{\mathbf{X}}^{c} \cap \mathcal{E}_{\mathbf{Z}}^{c}
$$

and therefore

$$
\operatorname{Pr}\left[\mathcal{E}_{\left(\hat{\mathbf{U}}_{1}, \hat{\mathbf{U}}_{2}\right)} \cap \mathcal{E}_{\mathbf{S}}^{c} \cap \mathcal{E}_{\mathbf{X}}^{c} \cap \mathcal{E}_{\mathbf{Z}}^{c}\right] \leq \operatorname{Pr}\left[\mathcal{E}_{\left(\hat{\mathbf{U}}_{1}, \hat{\mathbf{U}}_{2}\right)}^{\prime} \cap \mathcal{E}_{\mathbf{S}}^{c} \cap \mathcal{E}_{\mathbf{X}}^{c} \cap \mathcal{E}_{\mathbf{Z}}^{c}\right]
$$

We now state one more lemma that will be used for the proof of (105).

Lemma 20 For every $\Theta \in(0,1]$ and $\Delta \in(0,1]$, let the set $\mathcal{G}$ be given by

$$
\begin{aligned}
\mathcal{G}=\{ & \left(\mathbf{s}_{1}, \mathbf{s}_{2}, \mathcal{C}_{1}, \mathcal{C}_{2}, \mathcal{C}_{c}, \mathbf{z}\right): \exists \mathbf{u}_{1}(j) \in \mathcal{C}_{1} \backslash\left\{\mathbf{u}_{1}^{*}\right\} \text { and } \exists \mathbf{u}_{2}(l) \in \mathcal{C}_{2} \backslash\left\{\mathbf{u}_{2}^{*}\right\} \text { s.t. } \\
& \left.\cos \varangle\left(\mathbf{w}, a_{1,1} \mathbf{u}_{1}(j)+a_{2,1} \mathbf{u}_{2}(l)\right) \geq \Delta \text { and } \cos \varangle\left(\mathbf{u}_{1}(j), \mathbf{u}_{2}(l)\right) \geq \Theta\right\} .
\end{aligned}
$$

Then,

$$
R_{1}+R_{2}<-\frac{1}{2} \log \left(\left(1-\Theta^{2}\right)\left(1-\Delta^{2}\right)\right) \Longrightarrow\left(\lim _{n \rightarrow \infty} \operatorname{Pr}\left[\mathcal{G} \mid \mathcal{E}_{\mathbf{X}_{1}}^{c} \cap \mathcal{E}_{\mathbf{X}_{2}}^{c}\right]=0, \epsilon>0\right) .
$$

Proof: The proof follows from upper-bounding in every point on $\mathcal{S}_{1}, \mathcal{S}_{2}$ the density of every $\mathbf{u}_{1}(j) \in \mathcal{C}_{1} \backslash\left\{\mathbf{u}_{1}^{*}\right\}, \mathbf{u}_{2}(l) \in \mathcal{C}_{2} \backslash\left\{\mathbf{u}_{2}^{*}\right\}$ and then using a standard argument from sphere-packing.

Next,

$$
\begin{aligned}
\operatorname{Pr}\left[\mathcal{E}_{\left(\hat{\mathbf{U}}_{1}, \hat{\mathbf{U}}_{2}\right)} \cap \mathcal{E}_{\mathbf{S}}^{c} \cap \mathcal{E}_{\mathbf{X}}^{c} \cap \mathcal{E}_{\mathbf{Z}}^{c}\right]^{(a)} & \leq \operatorname{Pr}\left[\mathcal{E}_{\left(\hat{\mathbf{U}}_{1}, \hat{\mathbf{U}}_{2}\right)}^{\prime} \cap \mathcal{E}_{\mathbf{S}}^{c} \cap \mathcal{E}_{\mathbf{X}}^{c} \cap \mathcal{E}_{\mathbf{Z}}^{c}\right] \\
& \stackrel{(b)}{\leq} \operatorname{Pr}\left[\mathcal{E}^{\prime}{ }_{\left(\hat{\mathbf{U}}_{1}, \hat{\mathbf{U}}_{2}\right)} \mid \mathcal{E}_{\mathbf{X}_{1}}^{c} \cap \mathcal{E}_{\mathbf{X}_{2}}^{c}\right],
\end{aligned}
$$

where (a) follows by Lemma 19 and (b) follows because $\mathcal{E}_{\mathbf{X}}^{c} \subseteq \mathcal{E}_{\mathbf{X}_{1}}^{c} \cap \mathcal{E}_{\mathbf{X}_{2}}^{c}$.

The proof of (105) is now completed by combining (164) with Lemma 20. This gives that for every $\delta>0$ and every $\epsilon>0$ there exists some $n^{\prime}(\delta, \epsilon)$ such that for all $n>n^{\prime}(\delta, \epsilon)$, we have

whenever

$$
\operatorname{Pr}\left[\mathcal{E}_{\left(\hat{\mathbf{U}}_{1}, \hat{\mathbf{U}}_{2}\right)} \cap \mathcal{E}_{\mathbf{S}}^{c} \cap \mathcal{E}_{\mathbf{X}}^{c} \cap \mathcal{E}_{\mathbf{Z}}^{c}\right] \leq \operatorname{Pr}\left[\mathcal{E}_{\left(\hat{\mathbf{U}}_{1}, \hat{\mathbf{U}}_{2}\right)}^{\prime} \mid \mathcal{E}_{\mathbf{X}_{1}}^{c} \cap \mathcal{E}_{\mathbf{X}_{2}}^{c}\right]<\delta,
$$

$$
R_{1}+R_{2}<\frac{1}{2} \log \left(\frac{\lambda_{12}-\bar{\beta}_{2} P_{2} \bar{\rho}^{2}+N}{\left(1-\bar{\beta}_{2} P_{2} \bar{\rho}^{2} \lambda_{12}{ }^{-1}\right) N\left(1-\tilde{\rho}^{2}\right)}-\kappa_{4} \epsilon\right),
$$

where $\kappa_{4}$ is a positive constant determined by $P_{1}, P_{2}, N, \varsigma_{1}$ and $\varsigma_{2}$. 


\section{E. Proof of rate constraint (106)}

Define

$$
\mathbf{w}\left(\mathbf{s}_{1}, \mathbf{s}_{2}, \mathcal{C}_{1}, \mathcal{C}_{2}, \mathcal{C}_{c}, \mathbf{z}\right)=\varsigma_{1}\left(\mathbf{y}-a_{2,1} \mathbf{u}_{2}{ }^{*}\right)+\varsigma_{2} a_{2,1} \mathbf{u}_{2}{ }^{*},
$$

where

$$
\begin{aligned}
\varsigma_{1} & =\frac{\bar{\beta}_{1} P_{1}\left(1-\tilde{\rho}^{2}\right)+\left\|\alpha \mathbf{v}^{*}\right\|^{2}\left(1-\bar{\rho}^{2}\right)-2 \sqrt{\beta_{1} P_{1} \sigma^{2}\left(1-2^{-2 R_{1}}\right)} \alpha \bar{\rho}^{2}}{\bar{\beta}_{1} P_{1}\left(1-\tilde{\rho}^{2}\right)+\left\|\alpha \mathbf{v}^{*}\right\|^{2}\left(1-\bar{\rho}^{2}\right)-2 \sqrt{\bar{\beta}_{1} P_{1} \sigma^{2}\left(1-2^{-2 R_{1}}\right)} \alpha \bar{\rho}^{2}+N} \\
\varsigma_{2} & =\frac{\rho N\left(a_{1,1}\left(1-2^{-2 R_{1}}\right)+\alpha\left(1-2^{-2 R_{\mathrm{c}}}\right) 2^{-2 R_{1}}\right)}{a_{2,1}\left(\bar{\beta}_{1} P_{1}\left(1-\tilde{\rho}^{2}\right)+\left\|\alpha \mathbf{v}^{*}\right\|^{2}\left(1-\bar{\rho}^{2}\right)-2 \sqrt{\bar{\beta}_{1} P_{1} \sigma^{2}\left(1-2^{-2 R_{1}}\right)} \alpha \bar{\rho}^{2}+N\right)} .
\end{aligned}
$$

In the remainder we shall use the shorthand notation $\mathbf{w}$ instead of $\mathbf{w}\left(\mathbf{s}_{1}, \mathbf{s}_{2}, \mathcal{C}_{1}, \mathcal{C}_{2}, \mathcal{C}_{c}, \mathbf{z}\right)$. We now start with a lemma that will be used to prove (106).

Lemma 21 Let $\varphi_{j, l} \in[0, \pi]$ be the angle between $\mathbf{w}$ and $a_{1,1} \mathbf{u}_{1}(j)+\alpha \mathbf{v}(l)$, and let the set $\mathcal{E}_{\left(\hat{\mathbf{U}}_{1}, \hat{\mathbf{V}}\right)}^{\prime}$ be defined as

$$
\begin{gathered}
\mathcal{E}_{\left(\hat{\mathbf{U}}_{1}, \hat{\mathbf{V}}\right)}^{\prime} \triangleq\left\{\left(\mathbf{s}_{1}, \mathbf{s}_{2}, \mathcal{C}_{1}, \mathcal{C}_{2}, \mathcal{C}_{c}, \mathbf{z}\right): \exists \mathbf{u}_{1}(j) \in \mathcal{C}_{1} \backslash\left\{\mathbf{u}_{1}^{*}\right\} \text { and } \exists \mathbf{v}(j) \in \mathcal{C}_{c} \backslash\left\{\mathbf{v}^{*}\right\}\right. \text { s.t. } \\
\left.\cos \left(\varphi_{j}\right) \geq \sqrt{1-\frac{\Upsilon(\epsilon)}{n\left(\bar{\beta}_{1} P_{1}+\frac{1}{n}\|\alpha \mathbf{v}\|^{2}\right)}}\right\},
\end{gathered}
$$

where $\Upsilon(\epsilon)$ is defined in (175), and $\epsilon$ is sufficiently small such that the term inside the square is non-negative. Then,

$$
\mathcal{E}_{\left(\hat{\mathbf{U}}_{1}, \hat{\mathbf{V}}\right)} \cap \mathcal{E}_{\mathbf{S}}^{c} \cap \mathcal{E}_{\mathbf{X}}^{c} \cap \mathcal{E}_{\mathbf{Z}}^{c} \subseteq \mathcal{E}_{\left(\hat{\mathbf{U}}_{1}, \hat{\mathbf{V}}\right)}^{\prime} \cap \mathcal{E}_{\mathbf{S}}^{c} \cap \mathcal{E}_{\mathbf{X}}^{c} \cap \mathcal{E}_{\mathbf{Z}}^{c},
$$

and, in particular

$$
\operatorname{Pr}\left[\mathcal{E}_{\left(\hat{\mathbf{U}}_{1}, \hat{\mathbf{V}}\right)} \cap \mathcal{E}_{\mathbf{S}}^{c} \cap \mathcal{E}_{\mathbf{X}}^{c} \cap \mathcal{E}_{\mathbf{Z}}^{c}\right] \leq \operatorname{Pr}\left[\mathcal{E}_{\left(\hat{\mathbf{U}}_{1}, \hat{\mathbf{V}}\right)}^{\prime} \cap \mathcal{E}_{\mathbf{S}}^{c} \cap \mathcal{E}_{\mathbf{X}}^{c} \cap \mathcal{E}_{\mathbf{Z}}^{c}\right] .
$$

Proof: We first recall that for the event $\mathcal{E}_{\left(\hat{\mathbf{U}}_{1}, \hat{\mathbf{V}}\right)}$ to occur, there must exist codewords $\mathbf{u}_{1}(j) \in$ $\mathcal{C}_{1} \backslash\left\{\mathbf{u}_{1}^{*}\right\}$ and $\mathbf{v}(l) \in \mathcal{C}_{c} \backslash\left\{\mathbf{v}^{*}\right\}$ that satisfy the following four conditions

$$
\begin{aligned}
\left|\tilde{\rho}-\cos \varangle\left(\mathbf{u}_{1}(j), \mathbf{u}_{2}^{*}\right)\right| & \leq 7 \epsilon \\
\left|\bar{\rho}-\cos \varangle\left(\mathbf{v}(l), \mathbf{u}_{2}^{*}\right)\right| & \leq 7 \epsilon \\
\left|\cos \varangle\left(\mathbf{v}(l), \mathbf{u}_{1}(j)\right)\right| & \leq 7 \epsilon \\
\left\|\mathbf{y}-\mathbf{X}_{\mathbf{u}_{1}(j), \mathbf{v}(l), \mathbf{u}_{2}^{*}}\right\|^{2} & \leq\left\|\mathbf{y}-\mathbf{X}_{\mathbf{u}_{1}^{*}, \mathbf{v}^{*}, \mathbf{u}_{2}^{*}}\right\|^{2} .
\end{aligned}
$$

The proof is now based on a sequence of statements related to these conditions:

A) For every $\left(\mathbf{s}_{1}, \mathbf{s}_{2}, \mathcal{C}_{1}, \mathcal{C}_{2}, \mathcal{C}_{c}, \mathbf{z}\right) \in \mathcal{E}_{\mathbf{X}}^{c}$ and every $\mathbf{u}_{1} \in \mathcal{S}_{1}$ and $\mathbf{v} \in \mathcal{S}_{c}$,

$$
\left|\cos \varangle\left(\mathbf{u}_{1}, \mathbf{v}\right)\right| \leq 7 \epsilon \quad \Longrightarrow \quad\left|\left\langle a_{1,1} \mathbf{u}_{1}, \alpha \mathbf{v}\right\rangle\right| \leq 7 \sqrt{n \bar{\beta}_{1} P_{1}}\|\alpha \mathbf{v}\| \epsilon .
$$

Statement A) follows by rewriting $\cos \varangle\left(\mathbf{u}_{1}, \mathbf{v}\right)$ as $\left\langle\mathbf{u}_{1}, \mathbf{v}\right\rangle /\left(\left\|\mathbf{u}_{1}\right\|\|\mathbf{v}\|\right)$, and then multiplying the inequality on the 1.h.s. of (171) by $\left\|a_{1,1} \mathbf{u}_{1}\right\| \cdot\|\alpha \mathbf{v}\|$ and recalling that $\left\|a_{1,1} \mathbf{u}_{1}\right\|=\sqrt{n \bar{\beta}_{1} P_{1}}$. 
A1) For every $\left(\mathbf{s}_{1}, \mathbf{s}_{2}, \mathcal{C}_{1}, \mathcal{C}_{2}, \mathcal{C}_{c}, \mathbf{z}\right) \in \mathcal{E}_{\mathbf{X}}^{c}$ and every $\mathbf{u}_{1} \in \mathcal{S}_{1}$ and $\mathbf{v} \in \mathcal{S}_{c}$,

$$
\begin{aligned}
& \left|\bar{\rho}-\cos \varangle\left(\mathbf{v}, \mathbf{u}_{2}^{*}\right)\right| \leq 7 \epsilon \\
& \quad \Longrightarrow \quad|| \alpha \mathbf{v}\left\|\sqrt{n \overline{\beta_{2}} P_{2}} \bar{\rho}-\left\langle\alpha \mathbf{v}, a_{2,1} \mathbf{u}_{2}^{*}\right\rangle \mid \leq 7 \epsilon\right\| \alpha \mathbf{v} \| \sqrt{n \bar{\beta}_{2} P_{2}} .
\end{aligned}
$$

Statement A1) follows by rewriting $\cos \varangle\left(\mathbf{v}, \mathbf{u}_{2}^{*}\right)$ as $\left\langle\mathbf{v}, \mathbf{u}_{2}^{*}\right\rangle /\left(\|\mathbf{v}\|\left\|\mathbf{u}_{2}^{*}\right\|\right)$, and then multiplying the inequality on the 1.h.s. of (172) by $\|\alpha \mathbf{v}\| \cdot\left\|a_{2,1} \mathbf{u}_{2}^{*}\right\|$ and recalling that $\left\|a_{2,1} \mathbf{u}_{2}^{*}\right\|=\sqrt{n \bar{\beta}_{2} P_{2}}$.

B) For every $\left(\mathbf{s}_{1}, \mathbf{s}_{2}, \mathcal{C}_{1}, \mathcal{C}_{2}, \mathcal{C}_{c}, \mathbf{z}\right) \in \mathcal{E}_{\mathbf{X}}^{c} \cap \mathcal{E}_{\mathbf{Z}}^{c}$ and every $\mathbf{u}_{1} \in \mathcal{S}_{1}$ and $\mathbf{v} \in \mathcal{S}_{c}$

$$
\begin{aligned}
& \mid \mathbf{y}-\mathbf{X}_{\mathbf{u}_{1}, \mathbf{v}, \mathbf{u}_{2}^{*}}\left\|^{2} \leq\right\| \mathbf{y}-\mathbf{X}_{\mathbf{u}_{1}^{*}, \mathbf{v}^{*}, \mathbf{u}_{2}^{*}} \|^{2} \\
& \quad \Longrightarrow\left\langle\mathbf{y}-a_{2,1} \mathbf{u}_{2}^{*}, a_{1,1} \mathbf{u}_{1}+\alpha \mathbf{v}\right\rangle \geq n\left(\bar{\beta}_{1} P_{1}+\frac{1}{n}\left\|\alpha \mathbf{v}^{*}\right\|^{2}-\kappa \epsilon\right) .
\end{aligned}
$$

Statement B) follows from rewriting the inequality on the 1.h.s. of (173) as

$$
\left\|\left(\mathbf{y}-a_{2,1} \mathbf{u}_{2}^{*}\right)-\left(a_{1,1} \mathbf{u}_{1}+\alpha \mathbf{v}\right)\right\|^{2} \leq\left\|\left(\mathbf{y}-a_{2,1} \mathbf{u}_{2}^{*}\right)-\left(a_{1,1} \mathbf{u}_{1}^{*}+\alpha \mathbf{v}^{*}\right)\right\|^{2},
$$

or equivalently as

$$
\begin{aligned}
\left\langle\left(\mathbf{y}-a_{2,1} \mathbf{u}_{2}^{*}\right), a_{1,1} \mathbf{u}_{1}+\alpha \mathbf{v}\right\rangle & \geq\left\langle\left(\mathbf{y}-a_{2,1} \mathbf{u}_{2}^{*}\right), a_{1,1} \mathbf{u}_{1}^{*}+\alpha \mathbf{v}^{*}\right\rangle \\
& =\left\langle a_{1,1} \mathbf{u}_{1}^{*}+\alpha \mathbf{v}^{*}+\mathbf{z}, a_{1,1} \mathbf{u}_{1}^{*}+\alpha \mathbf{v}^{*}\right\rangle \\
& =\left\|a_{1,1} \mathbf{u}_{1}^{*}+\alpha \mathbf{v}^{*}\right\|^{2}+\left\langle\mathbf{z}, a_{1,1} \mathbf{u}_{1}^{*}+\alpha \mathbf{v}^{*}\right\rangle .
\end{aligned}
$$

It now follows from the equivalence of the first inequality in (173) with 174 that for $\left(\mathbf{s}_{1}, \mathbf{s}_{2}, \mathcal{C}_{1}, \mathcal{C}_{2}, \mathcal{C}_{c}, \mathbf{z}\right) \in \mathcal{E}_{\mathbf{Z}}^{c}$, the first inequality in (173) can only hold if

$$
\left\langle\mathbf{y}-a_{2,1} \mathbf{u}_{2}^{*}, a_{1,1} \mathbf{u}_{1}+\alpha \mathbf{v}\right\rangle \geq n\left(\bar{\beta}_{1} P_{1}+\frac{1}{n}\left\|\alpha \mathbf{v}^{*}\right\|^{2}-\kappa \epsilon\right),
$$

thus establishing B).

C) For every $\left(\mathbf{s}_{1}, \mathbf{s}_{2}, \mathcal{C}_{1}, \mathcal{C}_{2}, \mathcal{C}_{c}, \mathbf{z}\right) \in \mathcal{E}_{\mathbf{X}}^{c} \cap \mathcal{E}_{\mathbf{Z}}^{c}$ and every $\mathbf{u}_{1} \in \mathcal{S}_{1}$ and $\mathbf{v} \in \mathcal{S}_{c}$,

$$
\begin{aligned}
& \left|\tilde{\rho}-\cos \varangle\left(\mathbf{u}_{1}, \mathbf{u}_{2}^{*}\right)\right| \leq 7 \epsilon \text { and } \mid \mathbf{y}-\mathbf{X}_{\mathbf{u}_{1}, \mathbf{v}, \mathbf{u}_{2}^{*}}\left\|^{2} \leq\right\| \mathbf{y}-\mathbf{X}_{\mathbf{u}_{1}^{*}, \mathbf{v}^{*}, \mathbf{u}_{2}^{*}} \|^{2} \\
& \left\|a_{1,1} \mathbf{u}_{1}+\alpha \mathbf{v}-\mathbf{w}\right\|^{2} \leq n\left(\left(\bar{\beta}_{1} P_{1}+\frac{1}{n}\|\alpha \mathbf{v}\|^{2}\right)\left(1-2 \varsigma_{1}\right)-2 \varsigma_{2}\left(\sqrt{\bar{\beta}_{1} \bar{\beta}_{2} P_{1} P_{2}} \tilde{\rho}\right.\right. \\
& \left.\left.+\frac{1}{n}\left\|\alpha \mathbf{v}^{*}\right\| \sqrt{n \bar{\beta}_{2} P_{2}} \bar{\rho}\right)\right)+\|\mathbf{w}\|^{2}+n \kappa^{\prime} \epsilon .
\end{aligned}
$$

Statement C) is obtained as follows:

$$
\begin{aligned}
\left\|a_{1,1} \mathbf{u}_{1}+\alpha \mathbf{v}-\mathbf{w}\right\|^{2}= & \left\|a_{1,1} \mathbf{u}_{1}+\alpha \mathbf{v}\right\|^{2}-2\left\langle a_{1,1} \mathbf{u}_{1}+\alpha \mathbf{v}, \mathbf{w}\right\rangle+\|\mathbf{w}\|^{2} \\
= & \left\|a_{1,1} \mathbf{u}_{1}+\alpha \mathbf{v}\right\|^{2} \\
& -2\left\langle a_{1,1} \mathbf{u}_{1}+\alpha \mathbf{v}, \varsigma_{1}\left(a_{1,1} \mathbf{u}_{1}^{*}+\alpha \mathbf{v}^{*}+\mathbf{z}\right)+\varsigma_{2} a_{2,1} \mathbf{u}_{2}^{*}\right\rangle+\|\mathbf{w}\|^{2} \\
\stackrel{(a)}{\leq} & n\left(\left(\bar{\beta}_{1} P_{1}+\frac{1}{n}\|\alpha \mathbf{v}\|^{2}\right)\left(1-2 \varsigma_{1}\right)-2 \varsigma_{2}\left(\sqrt{\bar{\beta}_{1} \bar{\beta}_{2} P_{1} P_{2}} \tilde{\rho}\right.\right. \\
& \left.\left.+\frac{1}{n}\left\|\alpha \mathbf{v}^{*}\right\| \sqrt{n \bar{\beta}_{2} P_{2}} \bar{\rho}\right)\right)+\|\mathbf{w}\|^{2}+n \kappa^{\prime} \epsilon,
\end{aligned}
$$

where in (a) we have used Statement A) and Statement B). 
D) For every $\left(\mathbf{s}_{1}, \mathbf{s}_{2}, \mathcal{C}_{1}, \mathcal{C}_{2}, \mathcal{C}_{c}, \mathbf{z}\right) \in \mathcal{E}_{\mathbf{X}}^{c} \cap \mathcal{E}_{\mathbf{Z}}^{c}$

$$
\begin{aligned}
\|\mathbf{w}\|^{2} \leq & n\left(\varsigma_{1}^{2}\left(\bar{\beta}_{1} P_{1}+N+\frac{1}{n}\left\|\alpha \mathbf{v}^{*}\right\|^{2}\right)+2 \varsigma_{1} \varsigma_{2}\left(\sqrt{\bar{\beta}_{1} \bar{\beta}_{2} P_{1} P_{2}} \tilde{\rho}+\frac{1}{n}\left\|\alpha \mathbf{v}^{*}\right\| \sqrt{n \bar{\beta}_{2} P_{2}} \bar{\rho}\right)\right. \\
& \left.+\varsigma_{2}^{2} \bar{\beta}_{2} P_{2}+\kappa \epsilon\right)
\end{aligned}
$$

where $\kappa$ depends on $P_{1}, P_{2}, N, \varsigma_{1}$ and $\varsigma_{2}$ only.

Statement D) is obtained as follows:

$$
\begin{aligned}
\|\mathbf{w}\|^{2}= & \left\|\varsigma_{1}\left(a_{1,1} \mathbf{u}_{1}^{*}+\alpha \mathbf{v}^{*}+\mathbf{z}\right)+\varsigma_{2} a_{2,1} \mathbf{u}_{2}^{*}\right\|^{2} \\
= & \varsigma_{1}{ }^{2}\left\|a_{1,1} \mathbf{u}_{1}^{*}+\alpha \mathbf{v}^{*}+\mathbf{z}\right\|^{2}+2 \varsigma_{1} \varsigma_{2}\left\langle a_{1,1} \mathbf{u}_{1}^{*}+\alpha \mathbf{v}^{*}+\mathbf{z}, a_{2,1} \mathbf{u}_{2}^{*}\right\rangle+\varsigma_{2}{ }^{2}\left\|a_{2,1} \mathbf{u}_{2}^{*}\right\|^{2} \\
= & \varsigma_{1}{ }^{2}\left(\left\|a_{1,1} \mathbf{u}_{1}^{*}+\alpha \mathbf{v}^{*}\right\|^{2}+2\left\langle a_{1,1} \mathbf{u}_{1}^{*}+\alpha \mathbf{v}^{*}, \mathbf{z}\right\rangle+\|\mathbf{z}\|^{2}\right) \\
& +2 \varsigma_{1} \varsigma_{2}\left\langle a_{1,1} \mathbf{u}_{1}^{*}+\alpha \mathbf{v}^{*}+\mathbf{z}, a_{2,1} \mathbf{u}_{2}^{*}\right\rangle+\varsigma_{2}{ }^{2} n \bar{\beta}_{2} P_{2} \\
& \stackrel{(a)}{\leq} n\left(\varsigma_{1}{ }^{2}\left(\bar{\beta}_{1} P_{1}+N+\frac{1}{n}\left\|\alpha \mathbf{v}^{*}\right\|^{2}\right)+2 \varsigma_{1} \varsigma_{2}\left(\sqrt{\bar{\beta}_{1} \bar{\beta}_{2} P_{1} P_{2} \tilde{\rho}}+\frac{1}{n}\left\|\alpha \mathbf{v}^{*}\right\| \sqrt{n \bar{\beta}_{2} P_{2} \bar{\rho}}\right)\right. \\
& \left.+\varsigma_{2}{ }^{2} \bar{\beta}_{2} P_{2}+k \epsilon\right),
\end{aligned}
$$

where in (a) we have used that $\left(\mathbf{s}_{1}, \mathbf{s}_{2}, \mathcal{C}_{1}, \mathcal{C}_{2}, \mathcal{C}_{c}, \mathbf{z}\right) \in \mathcal{E}_{\mathbf{X}}^{c}$, and statements A) and A1).

E) For every $\left(\mathbf{s}_{1}, \mathbf{s}_{2}, \mathcal{C}_{1}, \mathcal{C}_{2}, \mathcal{C}_{c}, \mathbf{z}\right) \in \mathcal{E}_{\mathbf{X}}^{c} \cap \mathcal{E}_{\mathbf{Z}}^{c}$ and an arbitrary $\mathbf{u}_{1} \in \mathcal{S}_{1}$ and $\mathbf{v} \in \mathcal{S}_{c}$,

$$
\begin{aligned}
& \left|\tilde{\rho}-\cos \varangle\left(\mathbf{u}_{1}, \mathbf{u}_{2}^{*}\right)\right| \leq 7 \epsilon \text { and }\left|\bar{\rho}-\cos \varangle\left(\mathbf{v}, \mathbf{u}_{2}^{*}\right)\right| \leq 7 \epsilon \text { and } \mid \mathbf{y}-\mathbf{X}_{\mathbf{u}_{1}, \mathbf{v}, \mathbf{u}_{2}^{*}}\left\|^{2} \leq\right\| \mathbf{y}-\mathbf{X}_{\mathbf{u}_{1}^{*}, \mathbf{v}^{*}, \mathbf{u}_{2}^{*}} \|^{2} \\
& \Longrightarrow\left\|a_{1,1} \mathbf{u}_{1}+\alpha \mathbf{v}-\mathbf{w}\right\|^{2} \leq \Upsilon(\epsilon),
\end{aligned}
$$

where

$$
\Upsilon(\epsilon)=n \frac{\left(\bar{\beta}_{1} P_{1}\left(1-\tilde{\rho}^{2}\right)+\frac{1}{n}\|\alpha \mathbf{v}\|^{2}\left(1-\bar{\rho}^{2}\right)-2 \sqrt{\bar{\beta}_{1} P_{1} \sigma^{2}\left(1-2^{-2 R_{1}}\right)} \alpha \bar{\rho}^{2}\right) N}{\bar{\beta}_{1} P_{1}\left(1-\tilde{\rho}^{2}\right)+\frac{1}{n}\left\|\alpha \mathbf{v}^{*}\right\|^{2}\left(1-\bar{\rho}^{2}\right)-2 \sqrt{\beta_{1} P_{1} \sigma^{2}\left(1-2^{-2 R_{1}}\right)} \alpha \bar{\rho}^{2}+N}+n \kappa^{\prime} \epsilon,
$$

and where $\kappa^{\prime}$ only depends on $P_{1}, N_{1}, N_{2}, \varsigma_{1}$ and $\varsigma_{2}$.

Statement E) follows from combining Statement C) with Statement D) and the explicit values of $\varsigma_{1}$ and $\varsigma_{2}$ given in (165).

F) For every $\mathbf{u}_{1} \in \mathcal{S}_{1}, \mathbf{v} \in \mathcal{S}_{c}$, denote by $\varphi \in[0, \pi]$ the angle between $a_{1,1} \mathbf{u}_{1}+\alpha \mathbf{v}$ and $\mathbf{w}$, and let

$$
\mathcal{B}\left(\mathbf{s}_{1}, \mathbf{s}_{2}, \mathbf{u}_{1}^{*}, \mathbf{v}^{*}, \mathbf{u}_{2}^{*}, \mathbf{z}\right) \triangleq\left\{\mathbf{u}_{1} \in S_{1}^{(n)}, \mathbf{v} \in S_{c}^{(n)}: \quad \cos (\varphi) \geq \sqrt{1-\frac{\Upsilon(\epsilon)}{n\left(\bar{\beta}_{1} P_{1}+\frac{1}{n}\|\alpha \mathbf{v}\|^{2}\right)}}\right\},
$$

where $\epsilon$ is sufficiently small such that the term inside the square is non-negative. Then, for every $\left(\mathbf{s}_{1}, \mathbf{s}_{2}, \mathcal{C}_{1}, \mathcal{C}_{2}, \mathcal{C}_{c}, \mathbf{z}\right) \in \mathcal{E}_{\mathbf{X}}^{c} \cap \mathcal{E}_{\mathbf{Z}}^{c}$,

$$
\begin{aligned}
& \left|\tilde{\rho}-\cos \varangle\left(\mathbf{u}_{1}, \mathbf{u}_{2}^{*}\right)\right| \leq 7 \epsilon \quad \text { and } \quad\left|\bar{\rho}-\cos \varangle\left(\mathbf{v}, \mathbf{u}_{2}^{*}\right)\right| \leq 7 \epsilon \quad \text { and } \\
& \mid \mathbf{y}-\mathbf{X}_{\mathbf{u}_{1}, \mathbf{v}, \mathbf{u}_{2}^{*}}\left\|^{2} \leq\right\| \mathbf{y}-\mathbf{X}_{\mathbf{u}_{1}^{*}, \mathbf{v}^{*}, \mathbf{u}_{2}^{*}} \|^{2} \\
& \Longrightarrow \quad a_{1,1} \mathbf{u}_{1}+\alpha \mathbf{v} \in \mathcal{B}\left(\mathbf{s}_{1}, \mathbf{s}_{2}, \mathbf{u}_{1}^{*}, \mathbf{v}^{*}, \mathbf{u}_{2}^{*}, \mathbf{z}\right) .
\end{aligned}
$$


Statement F) follows from Statement E) by noting that if $\mathbf{w} \neq 0$ and

$$
1-\frac{\Upsilon(\epsilon)}{n\left(\bar{\beta}_{1} P_{1}+\frac{1}{n}\|\alpha \mathbf{v}\|^{2}\right)}>0,
$$

then

$$
\begin{gathered}
\left\|a_{1,1} \mathbf{u}_{1}+\alpha \mathbf{v}\right\|^{2}=n\left(\bar{\beta}_{1} P_{1}+\frac{1}{n}\|\alpha \mathbf{v}\|^{2}\right) \text { and }\left\|a_{1,1} \mathbf{u}_{1}+\alpha \mathbf{v}-\mathbf{w}\right\|^{2} \leq \Upsilon(\epsilon) \\
\Longrightarrow \cos \varangle\left(a_{1,1} \mathbf{u}_{1}+\alpha \mathbf{v}, \mathbf{w}\right) \geq \sqrt{1-\frac{\Upsilon(\epsilon)}{n\left(\bar{\beta}_{1} P_{1}+\frac{1}{n}\|\alpha \mathbf{v}\|^{2}\right)}},
\end{gathered}
$$

which follows by the same argument as 163).

The proof of Lemma 21 is now concluded by noticing that the set $\mathcal{E}_{\left(\hat{\mathbf{U}}_{1}, \hat{\mathbf{V}}\right)}$, defined in 166), is the set of tuples $\left(\mathbf{s}_{1}, \mathbf{s}_{2}, \mathcal{C}_{1}, \mathcal{C}_{2}, \mathcal{C}_{c}, \mathbf{z}\right)$ for which there exists a $\mathbf{u}_{1}(j) \in \mathcal{C}_{1} \backslash\left\{\mathbf{u}_{1}^{*}\right\}$ and $\mathbf{v}(l) \in$ $\mathcal{C}_{c} \backslash\left\{\mathbf{v}^{*}\right\}$ such that $a_{1,1} \mathbf{u}_{1}(j)+\alpha \mathbf{v}(l) \in \mathcal{B}\left(\mathbf{s}_{1}, \mathbf{s}_{2}, \mathbf{u}_{1}^{*}, \mathbf{v}^{*}, \mathbf{u}_{2}^{*}, \mathbf{z}\right)$. Thus, by Statement F) and by the definition of $\mathcal{E}_{\left(\hat{\mathbf{U}}_{1}, \hat{\mathbf{V}}\right)}$ in (91) it follows that

$$
\mathcal{E}_{\left(\hat{\mathbf{U}}_{1}, \hat{\mathbf{V}}\right)} \cap \mathcal{E}_{\mathbf{S}}^{c} \cap \mathcal{E}_{\mathbf{X}}^{c} \cap \mathcal{E}_{\mathbf{Z}}^{c} \subseteq \mathcal{E}_{\left(\hat{\mathbf{U}}_{1}, \hat{\mathbf{V}}\right)}^{\prime} \cap \mathcal{E}_{\mathbf{S}}^{c} \cap \mathcal{E}_{\mathbf{X}}^{c} \cap \mathcal{E}_{\mathbf{Z}}^{c}
$$

and therefore

$$
\operatorname{Pr}\left[\mathcal{E}_{\left(\hat{\mathbf{U}}_{1}, \hat{\mathbf{V}}\right)} \cap \mathcal{E}_{\mathbf{S}}^{c} \cap \mathcal{E}_{\mathbf{X}}^{c} \cap \mathcal{E}_{\mathbf{Z}}^{c}\right] \leq \operatorname{Pr}\left[\mathcal{E}_{\left(\hat{\mathbf{U}}_{1}, \hat{\mathbf{V}}\right)}^{\prime} \cap \mathcal{E}_{\mathbf{S}}^{c} \cap \mathcal{E}_{\mathbf{X}}^{c} \cap \mathcal{E}_{\mathbf{Z}}^{c}\right]
$$

Next,

$$
\begin{aligned}
\operatorname{Pr}\left[\mathcal{E}_{\left(\hat{\mathbf{U}}_{1}, \hat{\mathbf{V}}\right)} \cap \mathcal{E}_{\mathbf{S}}^{c} \cap \mathcal{E}_{\mathbf{X}}^{c} \cap \mathcal{E}_{\mathbf{Z}}^{c}\right] & \stackrel{(a)}{\leq} \operatorname{Pr}\left[\mathcal{E}_{\left(\hat{\mathbf{U}}_{1}, \hat{\mathbf{V}}\right)}^{\prime} \cap \mathcal{E}_{\mathbf{S}}^{c} \cap \mathcal{E}_{\mathbf{X}}^{c} \cap \mathcal{E}_{\mathbf{Z}}^{c}\right] \\
& \stackrel{(b)}{\leq} \operatorname{Pr}\left[\mathcal{E}_{\left(\hat{\mathbf{U}}_{1}, \hat{\mathbf{V}}\right)}^{\prime} \mid \mathcal{E}_{\mathbf{X}_{1}}^{c} \cap \mathcal{E}_{\mathbf{X}_{2}}^{c}\right]
\end{aligned}
$$

where (a) follows by Lemma 21 and (b) follows because $\mathcal{E}_{\mathbf{X}}^{c} \subseteq \mathcal{E}_{\mathbf{X}_{1}}^{c} \cap \mathcal{E}_{\mathbf{X}_{v}}^{c}$.

The proof of (106) is now completed by combining (177) with Lemma 20. This gives that for every $\delta>0$ and every $\epsilon>0$ there exists some $n^{\prime}(\delta, \epsilon)$ such that for all $n>n^{\prime}(\delta, \epsilon)$, we have

$$
\operatorname{Pr}\left[\mathcal{E}_{\left(\hat{\mathbf{U}}_{1}, \hat{\mathbf{V}}\right)} \cap \mathcal{E}_{\mathbf{S}}^{c} \cap \mathcal{E}_{\mathbf{X}}^{c} \cap \mathcal{E}_{\mathbf{Z}}^{c}\right] \leq \operatorname{Pr}\left[\mathcal{E}_{\left(\hat{\mathbf{U}}_{1}, \hat{\mathbf{V}}\right)}^{\prime} \mid \mathcal{E}_{\mathbf{X}_{1}}^{c} \cap \mathcal{E}_{\mathbf{X}_{v}}^{c}\right]<\delta
$$

whenever

$$
R_{1}+R_{\mathrm{c}}<\frac{1}{2} \log \left(\frac{\left(\lambda_{1 c}+N\right)\left(\bar{\beta}_{1} P_{1}+\eta^{2}\right)}{\lambda_{1 c} N}-\kappa_{5} \epsilon\right),
$$

where $\kappa_{5}$ is a positive constant determined by $P_{1}, P_{2}, N, \varsigma_{1}$ and $\varsigma_{2}$.

\section{F. Proof of rate constraint (107)}

Define

$$
\mathbf{w}\left(\mathbf{s}_{1}, \mathbf{s}_{2}, \mathcal{C}_{1}, \mathcal{C}_{2}, \mathcal{C}_{c}, \mathbf{z}\right)=\varsigma_{1}\left(\mathbf{y}-a_{1,1} \mathbf{u}_{1}{ }^{*}\right)+\varsigma_{2} a_{1,1} \mathbf{u}_{1}{ }^{*},
$$

where

$$
\begin{aligned}
\varsigma_{1} & =\frac{\bar{\beta}_{2} P_{2}\left(1-\tilde{\rho}^{2}\right)+2\|\alpha \mathbf{v}\| \sqrt{n \bar{\beta}_{2} P_{2}} \bar{\rho}+\|\alpha \mathbf{v}\|^{2}}{\bar{\beta}_{2} P_{2}\left(1-\tilde{\rho}^{2}\right)+2\|\alpha \mathbf{v}\| \sqrt{n \bar{\beta}_{2} P_{2}} \bar{\rho}+\|\alpha \mathbf{v}\|^{2}+N} \\
\varsigma_{2} & =\frac{a_{2,1} \rho\left(1-2^{-2 R_{2}}\right) N}{a_{1,1}\left(\bar{\beta}_{2} P_{2}\left(1-\tilde{\rho}^{2}\right)+2\|\alpha \mathbf{v}\| \sqrt{n \bar{\beta}_{2} P_{2}} \bar{\rho}+\|\alpha \mathbf{v}\|^{2}+N\right)} .
\end{aligned}
$$


In the remainder we shall use the shorthand notation $\mathbf{w}$ instead of $\mathbf{w}\left(\mathbf{s}_{1}, \mathbf{s}_{2}, \mathcal{C}_{1}, \mathcal{C}_{2}, \mathcal{C}_{c}, \mathbf{z}\right)$. We now start with a lemma that will be used to prove 107.

Lemma 22 Let $\varphi_{j, l} \in[0, \pi]$ be the angle between $\mathbf{w}$ and $a_{2,1} \mathbf{u}_{2}(j)+\alpha \mathbf{v}^{*}(l)$, and let the set $\mathcal{E}_{\left(\hat{\mathbf{U}}_{1}, \hat{\mathbf{V}}\right)}^{\prime}$ be defined as

$$
\begin{gathered}
\mathcal{E}_{\left(\hat{\mathbf{U}}_{2}, \hat{\mathbf{V}}\right)}^{\prime} \triangleq\left\{\left(\mathbf{s}_{1}, \mathbf{s}_{2}, \mathcal{C}_{1}, \mathcal{C}_{2}, \mathcal{C}_{c}, \mathbf{z}\right): \exists \mathbf{u}_{2}(j) \in \mathcal{C}_{2} \backslash\left\{\mathbf{u}_{2}^{*}\right\} \text { and } \exists \mathbf{v}(l) \in \mathcal{C}_{c} \backslash\left\{\mathbf{v}^{*}\right\}\right. \\
\left.\qquad \operatorname{\Upsilon os}\left(\varphi_{j, l}\right) \geq \sqrt{1-\frac{1 \epsilon)}{n\left(\bar{\beta}_{2} P_{2}+\frac{2}{n}\|\alpha \mathbf{v}\| \sqrt{n \bar{\beta}_{2} P_{2}} \bar{\rho}+\frac{1}{n}\|\alpha \mathbf{v}\|^{2}\right)}}\right\},
\end{gathered}
$$

where $\Upsilon(\epsilon)$ is defined in (189) and $\epsilon$ is sufficiently small such that the term inside the square is non-negative. Then,

$$
\mathcal{E}_{\left(\hat{\mathbf{U}}_{2}, \hat{\mathbf{V}}\right)} \cap \mathcal{E}_{\mathbf{S}}^{c} \cap \mathcal{E}_{\mathbf{X}}^{c} \cap \mathcal{E}_{\mathbf{Z}}^{c} \subseteq \mathcal{E}_{\left(\hat{\mathbf{U}}_{2}, \hat{\mathbf{V}}\right)}^{\prime} \cap \mathcal{E}_{\mathbf{S}}^{c} \cap \mathcal{E}_{\mathbf{X}}^{c} \cap \mathcal{E}_{\mathbf{Z}}^{c},
$$

and, in particular

$$
\operatorname{Pr}\left[\mathcal{E}_{\left(\hat{\mathbf{U}}_{2}, \hat{\mathbf{V}}\right)} \cap \mathcal{E}_{\mathbf{S}}^{c} \cap \mathcal{E}_{\mathbf{X}}^{c} \cap \mathcal{E}_{\mathbf{Z}}^{c}\right] \leq \operatorname{Pr}\left[\mathcal{E}_{\left(\hat{\mathbf{U}}_{2}, \hat{\mathbf{V}}\right)}^{\prime} \cap \mathcal{E}_{\mathbf{S}}^{c} \cap \mathcal{E}_{\mathbf{X}}^{c} \cap \mathcal{E}_{\mathbf{Z}}^{c}\right] .
$$

Proof: We first recall that for the event $\mathcal{E}_{\left(\hat{U}_{2}, \hat{V}\right)}$ to occur, there must exist codewords $\mathbf{u}_{2}(j) \in \mathcal{C}_{2} \backslash\left\{\mathbf{u}_{2}^{*}\right\}$ and $\mathbf{v}(l) \in \mathcal{C}_{c} \backslash\left\{\mathbf{v}^{*}\right\}$ that satisfy the following four conditions

$$
\begin{aligned}
\left|\tilde{\rho}-\cos \varangle\left(\mathbf{u}_{1}^{*}, \mathbf{u}_{2}(j)\right)\right| & \leq 7 \epsilon \\
\left|\bar{\rho}-\cos \varangle\left(\mathbf{v}(l), \mathbf{u}_{2}(j)\right)\right| & \leq 7 \epsilon \\
\left|\cos \varangle\left(\mathbf{v}(l), \mathbf{u}_{1}^{*}\right)\right| & \leq 3 \epsilon \\
\left\|\mathbf{y}-\mathbf{X}_{\mathbf{u}_{1}^{*}, \mathbf{v}(l), \mathbf{u}_{2}(j)}\right\|^{2} & \leq\left\|\mathbf{y}-\mathbf{X}_{\mathbf{u}_{1}^{*}, \mathbf{v}^{*}, \mathbf{u}_{2}^{*}}\right\|^{2} .
\end{aligned}
$$

The proof is now based on a sequence of statements related to these conditions:

A) For every $\left(\mathbf{s}_{1}, \mathbf{s}_{2}, \mathcal{C}_{1}, \mathcal{C}_{2}, \mathcal{C}_{c}, \mathbf{z}\right) \in \mathcal{E}_{X}^{c}$ and every $\mathbf{u}_{2} \in \mathcal{S}_{2}$ and $\mathbf{v} \in \mathcal{S}_{c}$,

$$
\left|\tilde{\rho}-\cos \varangle\left(\mathbf{u}_{1}^{*}, \mathbf{u}_{2}\right)\right| \leq 7 \epsilon \Longrightarrow\left|n \tilde{\rho} \sqrt{\bar{\beta}_{1} \bar{\beta}_{2} P_{1} P_{2}}-\left\langle a_{1,1} \mathbf{u}_{1}^{*}, a_{2,1} \mathbf{u}_{2}\right\rangle\right| \leq 7 n \sqrt{\bar{\beta}_{1} \bar{\beta}_{2} P_{1} P_{2}} \epsilon .
$$

Statement A) follows by rewriting $\cos \varangle\left(\mathbf{u}_{1}^{*}, \mathbf{u}_{2}\right)$ as $\left\langle\mathbf{u}_{1}^{*}, \mathbf{u}_{2}\right\rangle /\left(\left\|\mathbf{u}_{1}^{*}\right\|\left\|\mathbf{u}_{2}\right\|\right)$, and then multiplying the inequality on the 1.h.s. of (184) by $\left\|a_{1,1} \mathbf{u}_{1}^{*}\right\| \cdot\left\|a_{2,1} \mathbf{u}_{2}\right\|$.

A1) For every $\left(\mathbf{s}_{1}, \mathbf{s}_{2}, \mathcal{C}_{1}, \mathcal{C}_{2}, \mathcal{C}_{c}, \mathbf{z}\right) \in \mathcal{E}_{\mathbf{X}}^{c}$,

$$
\begin{aligned}
& \left|\cos \varangle\left(\mathbf{v}, \mathbf{u}_{1}^{*}\right)\right| \leq 3 \epsilon \\
& \quad \Longrightarrow \quad\left|\left\langle\alpha \mathbf{v}, a_{1,1} \mathbf{u}_{1}^{*}\right\rangle\right| \leq 3\|\alpha \mathbf{v}\| \sqrt{n \bar{\beta}_{1} P_{1}} \epsilon .
\end{aligned}
$$

Statement A1) follows by rewriting $\cos \varangle\left(\mathbf{v}, \mathbf{u}_{1}^{*}\right)$ as $\left\langle\mathbf{v}, \mathbf{u}_{1}^{*}\right\rangle /\left(\|\mathbf{v}\|\left\|\mathbf{u}_{1}^{*}\right\|\right)$, and then multiplying the inequality on the 1.h.s. of (185) by $\|\alpha \mathbf{v}\| \cdot\left\|a_{1,1} \mathbf{u}_{1}^{*}\right\|$ and recalling that $\left\|a_{1,1} \mathbf{u}_{1}^{*}\right\|=\sqrt{n \bar{\beta}_{1} P_{1}}$.

A2) For every $\left(\mathbf{s}_{1}, \mathbf{s}_{2}, \mathcal{C}_{1}, \mathcal{C}_{2}, \mathcal{C}_{c}, \mathbf{z}\right) \in \mathcal{E}_{\mathbf{X}}^{c}$ and every $\mathbf{u}_{2} \in \mathcal{S}_{2}$ and $\mathbf{v} \in \mathcal{S}_{c}$,

$$
\begin{aligned}
& \left|\bar{\rho}-\cos \varangle\left(\mathbf{v}, \mathbf{u}_{2}\right)\right| \leq 7 \epsilon \\
& \quad \Longrightarrow \quad|| \alpha \mathbf{v}\left\|\sqrt{n \bar{\beta}_{2} P_{2}} \bar{\rho}-\left\langle\alpha \mathbf{v}, a_{2,1} \mathbf{u}_{2}\right\rangle \mid \leq 7 \epsilon\right\| \alpha \mathbf{v} \| \sqrt{n \bar{\beta}_{2} P_{2}} .
\end{aligned}
$$

Statement A2) follows by rewriting $\cos \varangle\left(\mathbf{v}, \mathbf{u}_{2}\right)$ as $\left\langle\mathbf{v}, \mathbf{u}_{2}\right\rangle /\left(\|\mathbf{v}\|\left\|\mathbf{u}_{2}^{*}\right\|\right)$, and then multiplying the inequality on the 1.h.s. of (186) by $\|\alpha \mathbf{v}\| \cdot\left\|a_{2,1} \mathbf{u}_{2}\right\|$ and recalling that $\left\|a_{2,1} \mathbf{u}_{2}\right\|=\sqrt{n \bar{\beta}_{2} P_{2}}$. 
B) For every $\left(\mathbf{s}_{1}, \mathbf{s}_{2}, \mathcal{C}_{1}, \mathcal{C}_{2}, \mathcal{C}_{c}, \mathbf{z}\right) \in \mathcal{E}_{\mathbf{X}}^{c} \cap \mathcal{E}_{\mathbf{Z}}^{c}$ and every $\mathbf{u}_{2} \in \mathcal{S}_{2}$ and $\mathbf{v} \in \mathcal{S}_{c}$

$$
\begin{aligned}
& \mid \mathbf{y}-\mathbf{X}_{\mathbf{u}_{1}^{*}, \mathbf{v}, \mathbf{u}_{2}}\left\|^{2} \leq\right\| \mathbf{y}-\mathbf{X}_{\mathbf{u}_{1}^{*}, \mathbf{v}^{*}, \mathbf{u}_{2}^{*}} \|^{2} \\
& \Longrightarrow\left\langle\mathbf{y}-a_{1,1} \mathbf{u}_{1}^{*}, a_{2,1} \mathbf{u}_{2}+\alpha \mathbf{v}\right\rangle \geq n\left(\bar{\beta}_{2} P_{2}+\frac{2}{n}\|\alpha \mathbf{v}\| \sqrt{n \bar{\beta}_{2} P_{2}} \bar{\rho}+\frac{1}{n}\left\|\alpha \mathbf{v}^{*}\right\|^{2}-\kappa \epsilon\right) .
\end{aligned}
$$

Statement B) follows from rewriting the inequality on the 1.h.s. of 187) as

$$
\left\|\left(\mathbf{y}-a_{1,1} \mathbf{u}_{1}^{*}\right)-\left(a_{2,1} \mathbf{u}_{2}+\alpha \mathbf{v}\right)\right\|^{2} \leq\left\|\left(\mathbf{y}-a_{1,1} \mathbf{u}_{1}^{*}\right)-\left(a_{2,1} \mathbf{u}_{1}^{*}+\alpha \mathbf{v}^{*}\right)\right\|^{2},
$$

or equivalently as

$$
\begin{aligned}
\left\langle\left(\mathbf{y}-a_{1,1} \mathbf{u}_{1}^{*}\right), a_{2,1} \mathbf{u}_{2}+\alpha \mathbf{v}\right\rangle & \geq\left\langle\left(\mathbf{y}-a_{1,1} \mathbf{u}_{1}^{*}\right), a_{2,1} \mathbf{u}_{2}^{*}+\alpha \mathbf{v}^{*}\right\rangle \\
& =\left\langle a_{2,1} \mathbf{u}_{2}^{*}+\alpha \mathbf{v}^{*}+\mathbf{z}, a_{2,1} \mathbf{u}_{2}^{*}+\alpha \mathbf{v}^{*}\right\rangle \\
& =\left\|a_{2,1} \mathbf{u}_{2}^{*}+\alpha \mathbf{v}^{*}\right\|^{2}+\left\langle\mathbf{z}, a_{2,1} \mathbf{u}_{2}^{*}+\alpha \mathbf{v}^{*}\right\rangle .
\end{aligned}
$$

It now follows from the equivalence of the first inequality in (187) with 188 that for $\left(\mathbf{s}_{1}, \mathbf{s}_{2}, \mathcal{C}_{1}, \mathcal{C}_{2}, \mathcal{C}_{c}, \mathbf{z}\right) \in \mathcal{E}_{\mathbf{Z}}^{c}$, the first inequality in (187) can only hold if

$$
\left\langle\mathbf{y}-a_{1,1} \mathbf{u}_{1}^{*}, a_{2,1} \mathbf{u}_{2}+\alpha \mathbf{v}\right\rangle \geq n\left(\bar{\beta}_{2} P_{2}+\frac{2}{n}\|\alpha \mathbf{v}\| \sqrt{n \bar{\beta}_{2} P_{2}} \bar{\rho}+\frac{1}{n}\left\|\alpha \mathbf{v}^{*}\right\|^{2}-\kappa \epsilon\right),
$$

thus establishing B).

C) For every $\left(\mathbf{s}_{1}, \mathbf{s}_{2}, \mathcal{C}_{1}, \mathcal{C}_{2}, \mathcal{C}_{c}, \mathbf{z}\right) \in \mathcal{E}_{\mathbf{X}}^{c} \cap \mathcal{E}_{\mathbf{Z}}^{c}$ and every $\mathbf{u}_{2} \in \mathcal{S}_{2}$ and $\mathbf{v} \in \mathcal{S}_{c}$,

$$
\begin{aligned}
& \left|\tilde{\rho}-\cos \varangle\left(\mathbf{u}_{1}^{*}, \mathbf{u}_{2}\right)\right| \leq 7 \epsilon \text { and } \mid \mathbf{y}-\mathbf{X}_{\mathbf{u}_{1}^{*}, \mathbf{v}, \mathbf{u}_{2}}\left\|^{2} \leq\right\| \mathbf{y}-\mathbf{X}_{\mathbf{u}_{1}^{*}, \mathbf{v}^{*}, \mathbf{u}_{2}^{*}} \|^{2} \\
& \left\|a_{2,1} \mathbf{u}_{2}+\alpha \mathbf{v}-\mathbf{w}\right\|^{2} \leq n\left(\left(\bar{\beta}_{2} P_{2}+\frac{2}{n}\|\alpha \mathbf{v}\| \sqrt{n \bar{\beta}_{2} P_{2}} \bar{\rho}+\frac{1}{n}\|\alpha \mathbf{v}\|^{2}\right)\left(1-2 \varsigma_{1}\right)\right. \\
& \left.-2 \varsigma_{2} \sqrt{\bar{\beta}_{1} \bar{\beta}_{2} P_{1} P_{2}} \tilde{\rho}\right)+\|\mathbf{w}\|^{2}+n \kappa^{\prime} \epsilon .
\end{aligned}
$$

Statement C) is obtained as follows:

$$
\begin{aligned}
\left\|a_{2,1} \mathbf{u}_{2}+\alpha \mathbf{v}-\mathbf{w}\right\|^{2}= & \left\|a_{2,1} \mathbf{u}_{2}+\alpha \mathbf{v}\right\|^{2}-2\left\langle a_{2,1} \mathbf{u}_{2}+\alpha \mathbf{v}, \mathbf{w}\right\rangle+\|\mathbf{w}\|^{2} \\
= & \left\|a_{2,1} \mathbf{u}_{2}+\alpha \mathbf{v}\right\|^{2} \\
& -2\left\langle a_{2,1} \mathbf{u}_{2}+\alpha \mathbf{v}, \varsigma_{1}\left(a_{2,1} \mathbf{u}_{2}^{*}+\alpha \mathbf{v}^{*}+\mathbf{z}\right)+\varsigma_{2} a_{1,1} \mathbf{u}_{1}^{*}\right\rangle+\|\mathbf{w}\|^{2} \\
\stackrel{(a)}{\leq} & n\left(\left(\bar{\beta}_{2} P_{2}+\frac{2}{n}\|\alpha \mathbf{v}\| \sqrt{n \bar{\beta}_{2} P_{2}} \bar{\rho}+\frac{1}{n}\|\alpha \mathbf{v}\|^{2}\right)\left(1-2 \varsigma_{1}\right)\right. \\
& \left.-2 \varsigma_{2} \sqrt{\bar{\beta}_{1} \bar{\beta}_{2} P_{1} P_{2}} \tilde{\rho}\right)+\|\mathbf{w}\|^{2}+n \kappa^{\prime} \epsilon
\end{aligned}
$$

where in (a) we have used Statement A), A1) and Statement B).

D) For every $\left(\mathbf{s}_{1}, \mathbf{s}_{2}, \mathcal{C}_{1}, \mathcal{C}_{2}, \mathcal{C}_{c}, \mathbf{z}\right) \in \mathcal{E}_{\mathbf{X}}^{c} \cap \mathcal{E}_{\mathbf{Z}}^{c}$

$$
\begin{aligned}
\|\mathbf{w}\|^{2} \leq & n\left(\varsigma_{1}^{2}\left(\bar{\beta}_{2} P_{2}+N\right)+\varsigma_{1}^{2}\left(\frac{2}{n}\left\|\alpha \mathbf{v}^{*}\right\| \sqrt{n \bar{\beta}_{2} P_{2}} \bar{\rho}+\frac{1}{n}\left\|\alpha \mathbf{v}^{*}\right\|^{2}\right)\right. \\
& \left.+2 \varsigma_{1} \varsigma_{2} \sqrt{\overline{\beta_{1}} P_{1} \bar{\beta}_{2} P_{2}} \tilde{\rho}+\varsigma_{2}^{2} \bar{\beta}_{1} P_{1}+\kappa \epsilon\right)
\end{aligned}
$$

where $k$ depends on $P_{1}, P_{2}, N, \varsigma_{1}$ and $\varsigma_{2}$ only. 
Statement D) is obtained as follows:

$$
\begin{aligned}
\|\mathbf{w}\|^{2}= & \left\|\varsigma_{1}\left(a_{2,1} \mathbf{u}_{2}^{*}+\alpha \mathbf{v}^{*}+\mathbf{z}\right)+\varsigma_{2} a_{1,1} \mathbf{u}_{1}^{*}\right\|^{2} \\
= & \varsigma_{1}{ }^{2}\left\|a_{2,1} \mathbf{u}_{2}^{*}+\alpha \mathbf{v}^{*}+\mathbf{z}\right\|^{2}+2 \varsigma_{1} \varsigma_{2}\left\langle a_{2,1} \mathbf{u}_{2}^{*}+\alpha \mathbf{v}^{*}+\mathbf{z}, a_{1,1} \mathbf{u}_{1}^{*}\right\rangle+\varsigma_{2}{ }^{2}\left\|a_{1,1} \mathbf{u}_{1}^{*}\right\|^{2} \\
= & \varsigma_{1}{ }^{2}\left(\left\|a_{2,1} \mathbf{u}_{2}^{*}+\alpha \mathbf{v}^{*}\right\|^{2}+2\left\langle a_{2,1} \mathbf{u}_{2}^{*}+\alpha \mathbf{v}^{*}, \mathbf{z}\right\rangle+\|\mathbf{z}\|^{2}\right) \\
& +2 \varsigma_{1} \varsigma_{2}\left\langle a_{2,1} \mathbf{u}_{2}^{*}+\alpha \mathbf{v}^{*}+\mathbf{z}, a_{1,1} \mathbf{u}_{1}^{*}\right\rangle+\varsigma_{2}{ }^{2} n \bar{\beta}_{1} P_{1} \\
\stackrel{(a)}{\leq} & n\left(\varsigma_{1}{ }^{2}\left(\bar{\beta}_{2} P_{2}+N\right)+\varsigma_{1}{ }^{2}\left(\frac{2}{n}\|\alpha \mathbf{v}\| \sqrt{n \bar{\beta}_{2} P_{2}} \bar{\rho}+\frac{1}{n}\left\|\alpha \mathbf{v}^{*}\right\|^{2}\right)\right. \\
& \left.+2 \varsigma_{1} \varsigma_{2} \sqrt{\bar{\beta}_{1} P_{1} \bar{\beta}_{2} P_{2}} \tilde{\rho}+\varsigma_{2}{ }^{2} \bar{\beta}_{1} P_{1}+k \epsilon\right),
\end{aligned}
$$

where in (a) we have used that $\left(\mathbf{s}_{1}, \mathbf{s}_{2}, \mathcal{C}_{1}, \mathcal{C}_{2}, \mathcal{C}_{c}, \mathbf{z}\right) \in \mathcal{E}_{\mathbf{X}}^{c}$, and statements A) and A2).

E) For every $\left(\mathbf{s}_{1}, \mathbf{s}_{2}, \mathcal{C}_{1}, \mathcal{C}_{2}, \mathcal{C}_{c}, \mathbf{z}\right) \in \mathcal{E}_{\mathbf{X}}^{c} \cap \mathcal{E}_{\mathbf{Z}}^{c}$ and an arbitrary $\mathbf{u}_{2} \in \mathcal{S}_{2}$ and $\mathbf{v} \in \mathcal{S}_{c}$,

$$
\begin{aligned}
& \left|\tilde{\rho}-\cos \varangle\left(\mathbf{u}_{1}^{*}, \mathbf{u}_{2}\right)\right| \leq 7 \epsilon \text { and }\left|\bar{\rho}-\cos \varangle\left(\mathbf{v}, \mathbf{u}_{2}\right)\right| \leq 7 \epsilon \text { and } \mid \mathbf{y}-\mathbf{X}_{\mathbf{u}_{1}^{*}, \mathbf{v}, \mathbf{u}_{2}}\left\|^{2} \leq\right\| \mathbf{y}-\mathbf{X}_{\mathbf{u}_{1}^{*}, \mathbf{v}^{*}, \mathbf{u}_{2}^{*}} \|^{2} \\
& \Longrightarrow\left\|a_{2,1} \mathbf{u}_{2}+\alpha \mathbf{v}-\mathbf{w}\right\|^{2} \leq \Upsilon(\epsilon),
\end{aligned}
$$

where

$$
\Upsilon(\epsilon)=n \frac{\left(\bar{\beta}_{2} P_{2}\left(1-\tilde{\rho}^{2}\right)+\frac{2}{n}\|\alpha \mathbf{v}\| \sqrt{n \bar{\beta}_{2} P_{2}} \bar{\rho}+\frac{1}{n}\|\alpha \mathbf{v}\|^{2}\right) N}{\bar{\beta}_{2} P_{2}\left(1-\tilde{\rho}^{2}\right)+\frac{2}{n}\|\alpha \mathbf{v}\| \sqrt{n \bar{\beta}_{2} P_{2}} \bar{\rho}+\frac{1}{n}\|\alpha \mathbf{v}\|^{2}+N}+n \kappa^{\prime} \epsilon,
$$

and where $\kappa^{\prime}$ only depends on $P_{1}, N_{1}, N_{2}, \varsigma_{1}$ and $\varsigma_{2}$.

Statement E) follows from combining Statement C) with Statement D) and the explicit values of $\varsigma_{1}$ and $\varsigma_{2}$ given in (178).

F) For every $\mathbf{u}_{2} \in \mathcal{S}_{2}, \mathbf{v} \in \mathcal{S}_{c}$, denote by $\varphi \in[0, \pi]$ the angle between $a_{2,1} \mathbf{u}_{2}+\alpha \mathbf{v}$ and $\mathbf{w}$, and let

$$
\begin{aligned}
\mathcal{B}\left(\mathbf{s}_{1}, \mathbf{s}_{2}, \mathbf{u}_{1}^{*}, \mathbf{v}^{*}, \mathbf{u}_{2}^{*}, \mathbf{z}\right) \triangleq & \left\{\mathbf{u}_{2} \in S_{2}^{(n)}, \mathbf{v} \in S_{c}^{(n)}:\right. \\
& \left.\quad \cos (\varphi) \geq \sqrt{1-\frac{\Upsilon(\epsilon)}{n\left(\bar{\beta}_{2} P_{2}+\frac{2}{n}\|\alpha \mathbf{v}\| \sqrt{n \bar{\beta}_{2} P_{2}} \bar{\rho}+\frac{1}{n}\|\alpha \mathbf{v}\|^{2}\right)}}\right\},
\end{aligned}
$$

where $\epsilon$ is sufficiently small such that the term in the square is non-negative.

Then, for every $\left(\mathbf{s}_{1}, \mathbf{s}_{2}, \mathcal{C}_{1}, \mathcal{C}_{2}, \mathcal{C}_{c}, \mathbf{z}\right) \in \mathcal{E}_{\mathbf{X}}^{c} \cap \mathcal{E}_{\mathbf{Z}}^{c}$,

$$
\begin{aligned}
& \left|\tilde{\rho}-\cos \varangle\left(\mathbf{u}_{1}^{*}, \mathbf{u}_{2}\right)\right| \leq 7 \epsilon \quad \text { and } \quad\left|\bar{\rho}-\cos \varangle\left(\mathbf{v}, \mathbf{u}_{2}\right)\right| \leq 7 \epsilon \quad \text { and } \\
& \mid \mathbf{y}-\mathbf{X}_{\mathbf{u}_{1}^{*}, \mathbf{v}, \mathbf{u}_{2}}\left\|^{2} \leq\right\| \mathbf{y}-\mathbf{X}_{\mathbf{u}_{1}^{*}, \mathbf{v}^{*}, \mathbf{u}_{2}^{*}} \|^{2} \\
& \quad \Longrightarrow \quad a_{2,1} \mathbf{u}_{2}+\alpha \mathbf{v} \in \mathcal{B}\left(\mathbf{s}_{1}, \mathbf{s}_{2}, \mathbf{u}_{1}^{*}, \mathbf{v}^{*}, \mathbf{u}_{2}^{*}, \mathbf{z}\right) .
\end{aligned}
$$

Statement F) follows from Statement $\mathrm{E}$ ) by noting that if $\mathbf{w} \neq 0$ and

$$
1-\frac{\Upsilon(\epsilon)}{n\left(\bar{\beta}_{2} P_{2}+\frac{2}{n}\|\alpha \mathbf{v}\| \sqrt{n \bar{\beta}_{2} P_{2}} \bar{\rho}+\frac{1}{n}\|\alpha \mathbf{v}\|^{2}\right)}>0,
$$

then

$$
\begin{aligned}
& \left\|a_{2,1} \mathbf{u}_{2}+\alpha \mathbf{v}\right\|^{2}=n\left(\bar{\beta}_{2} P_{2}+\frac{2}{n}\|\alpha \mathbf{v}\| \sqrt{n \bar{\beta}_{2} P_{2}} \bar{\rho}+\frac{1}{n}\|\alpha \mathbf{v}\|^{2}\right) \\
& \text { and }\left\|a_{2,1} \mathbf{u}_{2}+\alpha \mathbf{v}-\mathbf{w}\right\|^{2} \leq \Upsilon(\epsilon)
\end{aligned}
$$




$$
\Longrightarrow \cos \varangle\left(a_{2,1} \mathbf{u}_{2}+\alpha \mathbf{v}, \mathbf{w}\right) \geq \sqrt{1-\frac{\Upsilon(\epsilon)}{n\left(\bar{\beta}_{2} P_{2}+\frac{2}{n}\|\alpha \mathbf{v}\| \sqrt{n \bar{\beta}_{2} P_{2}} \bar{\rho}+\frac{1}{n}\|\alpha \mathbf{v}\|^{2}\right)}},
$$

which follows by the same argument as (163).

The proof of Lemma 22 is now concluded by noticing that the set $\mathcal{E}_{\left(\hat{U}_{2}, \hat{V}\right)}$, defined in [179], is the set of tuples $\left(\mathbf{s}_{1}, \mathbf{s}_{2}, \mathcal{C}_{1}, \mathcal{C}_{2}, \mathcal{C}_{c}, \mathbf{z}\right)$ for which there exists a $\mathbf{u}_{2}(j) \in \mathcal{C}_{2} \backslash\left\{\mathbf{u}_{1}^{*}\right\}$ and $\mathbf{v}(l) \in$ $\mathcal{C}_{c} \backslash\left\{\mathbf{v}^{*}\right\}$ such that $a_{2,1} \mathbf{u}_{2}(j)+\alpha \mathbf{v}(l) \in B\left(\mathbf{s}_{1}, \mathbf{s}_{2}, \mathbf{u}_{1}^{*}, \mathbf{v}^{*}, \mathbf{u}_{2}^{*}, \mathbf{z}\right)$. Thus, by Statement F) and by the definition of $\mathcal{E}_{\left(\hat{\mathbf{U}}_{2}, \hat{\mathbf{v}}\right)}$ in 92$)$ it follows that

$$
\mathcal{E}_{\left(\hat{\mathbf{U}}_{2}, \hat{\mathbf{V}}\right)} \cap \mathcal{E}_{\mathbf{S}}^{c} \cap \mathcal{E}_{\mathbf{X}}^{c} \cap \mathcal{E}_{\mathbf{Z}}^{c} \subseteq \mathcal{E}_{\left(\hat{\mathbf{U}}_{2}, \hat{\mathbf{V}}\right)}^{\prime} \cap \mathcal{E}_{\mathbf{S}}^{c} \cap \mathcal{E}_{\mathbf{X}}^{c} \cap \mathcal{E}_{\mathbf{Z}}^{c}
$$

and therefore

$$
\operatorname{Pr}\left[\mathcal{E}_{\left(\hat{\mathbf{U}}_{2}, \hat{\mathbf{V}}\right)} \cap \mathcal{E}_{\mathbf{S}}^{c} \cap \mathcal{E}_{\mathbf{X}}^{c} \cap \mathcal{E}_{\mathbf{Z}}^{c}\right] \leq \operatorname{Pr}\left[\mathcal{E}_{\left(\hat{\mathbf{U}}_{2}, \hat{\mathbf{V}}\right)}^{\prime} \cap \mathcal{E}_{\mathbf{S}}^{c} \cap \mathcal{E}_{\mathbf{X}}^{c} \cap \mathcal{E}_{\mathbf{Z}}^{c}\right]
$$

Next,

$$
\begin{aligned}
\operatorname{Pr}\left[\mathcal{E}_{\left(\hat{\mathbf{U}}_{2}, \hat{\mathbf{V}}\right)} \cap \mathcal{E}_{\mathbf{S}}^{c} \cap \mathcal{E}_{\mathbf{X}}^{c} \cap \mathcal{E}_{\mathbf{Z}}^{c}\right] & \stackrel{(a)}{\leq} \operatorname{Pr}\left[\mathcal{E}_{\left(\hat{\mathbf{U}}_{2}, \hat{\mathbf{V}}\right)}^{\prime} \cap \mathcal{E}_{\mathbf{S}}^{c} \cap \mathcal{E}_{\mathbf{X}}^{c} \cap \mathcal{E}_{\mathbf{Z}}^{c}\right] \\
& \stackrel{(b)}{\leq} \operatorname{Pr}\left[\mathcal{E}_{\left(\hat{\mathbf{U}}_{2}, \hat{\mathbf{V}}\right)}^{\prime} \mid \mathcal{E}_{\mathbf{X}_{2}}^{c} \cap \mathcal{E}_{\mathbf{X}_{v}}^{c}\right],
\end{aligned}
$$

where (a) follows by Lemma 22 and (b) follows because $\mathcal{E}_{\mathbf{X}}^{c} \subseteq \mathcal{E}_{\mathbf{X}_{2}}^{c} \cap \mathcal{E}_{\mathbf{X}_{v}}^{c}$.

The proof of (107) is now completed by combining (191) with Lemma20. This gives that for every $\delta>0$ and every $\epsilon>0$ there exists some $n^{\prime}(\delta, \epsilon)$ such that for all $n>n^{\prime}(\delta, \epsilon)$, we have

$$
\operatorname{Pr}\left[\mathcal{E}_{\left(\hat{\mathbf{U}}_{2}, \hat{\mathbf{V}}\right)} \cap \mathcal{E}_{\mathbf{S}}^{c} \cap \mathcal{E}_{\mathbf{X}}^{c} \cap \mathcal{E}_{\mathbf{Z}}^{c}\right] \leq \operatorname{Pr}\left[\mathcal{E}_{\left(\hat{\mathbf{U}}_{2}, \hat{\mathbf{V}}\right)}^{\prime} \mid \mathcal{E}_{\mathbf{X}_{2}}^{c} \cap \mathcal{E}_{\mathbf{X}_{v}}^{c}\right]<\delta,
$$

whenever

$$
R_{2}+R_{\mathrm{c}}<\frac{1}{2} \log \left(\frac{\lambda_{2 c}-\bar{\beta}_{2} P_{2} \tilde{\rho}^{2}+N}{\left(1-\bar{\beta}_{2} P_{2} \tilde{\rho}^{2} \lambda_{2 c}{ }^{-1}\right) N\left(1-\bar{\rho}^{2}\right)}-\kappa_{6} \epsilon\right),
$$

where $\kappa_{6}$ is a positive constant determined by $P_{1}, P_{2}, N, \varsigma_{1}$ and $\varsigma_{2}$.

\section{G. Proof of rate constraint (108)}

Lemma 23 For every sufficiently small $\epsilon>0$, define the $\operatorname{set}_{\left(\hat{\mathbf{U}}_{1}, \hat{\mathbf{U}}_{2}, \hat{\mathbf{V}}\right)}^{\prime}$ as

$\mathcal{E}_{\left(\hat{\mathbf{U}}_{1}, \hat{\mathbf{U}}_{2}, \hat{\mathbf{v}}\right)}^{\prime} \triangleq\left\{\left(\mathbf{s}_{1}, \mathbf{s}_{2}, \mathcal{C}_{1}, \mathcal{C}_{2}, \mathcal{C}_{c}, \mathbf{z}\right): \exists \mathbf{u}_{1}(j) \in \mathcal{C}_{1} \backslash\left\{\mathbf{u}_{1}^{*}\right\}\right.$ and $\exists \mathbf{u}_{2}(l) \in \mathcal{C}_{2} \backslash\left\{\mathbf{u}_{2}^{*}\right\}$

and $\exists \mathbf{v}(k) \in \mathcal{C}_{c} \backslash\left\{\mathbf{v}^{*}\right\}$ s.t. $\cos \varangle\left(\mathbf{u}_{1}(j), \mathbf{u}_{2}(l)\right) \geq \tilde{\rho}-7 \epsilon$ and $\cos \varangle\left(\mathbf{u}_{1}(j), \mathbf{v}(k)\right) \geq-3 \epsilon$

and $\cos \varangle\left(\mathbf{v}(k), \mathbf{u}_{2}(l)\right) \geq \bar{\rho}-7 \epsilon$ and $\left.\cos \varangle\left(\mathbf{y}, a_{1,1} \mathbf{u}_{1}(j)+a_{2,1} \mathbf{u}_{2}(l)+\alpha \mathbf{v}(k)\right) \geq \Lambda(\epsilon)\right\}$,

where

$$
\Lambda(\epsilon)=\sqrt{\frac{\bar{\beta}_{1} P_{1}+2 \sqrt{\bar{\beta}_{1} P_{1} \bar{\beta}_{2} P_{2}} \tilde{\rho}+\bar{\beta}_{2} P_{2}+2 \frac{1}{n}\left\|\alpha \mathbf{v}^{*}\right\| \sqrt{n \bar{\beta}_{2} P_{2}} \bar{\rho}+\frac{1}{n}\left\|\alpha \mathbf{v}^{*}\right\|^{2}-\xi^{\prime} \epsilon}{\bar{\beta}_{1} P_{1}+2 \sqrt{\bar{\beta}_{1} P_{1} \bar{\beta}_{2} P_{2}} \tilde{\rho}+\bar{\beta}_{2} P_{2}+\frac{2}{n}\left\|\alpha \mathbf{v}^{*}\right\| \sqrt{n \bar{\beta}_{2} P_{2}} \bar{\rho}+\frac{1}{n}\left\|\alpha \mathbf{v}^{*}\right\|^{2}+N+\xi_{2} \epsilon}},
$$

and where $\xi^{\prime}$ and $\xi_{2}$ depend only on $P_{1}, P_{2}$ and $N$. Then, for sufficiently small $\epsilon>0$

$$
\mathcal{E}_{\left(\hat{\mathbf{U}}_{1}, \hat{\mathbf{U}}_{2}, \hat{\mathbf{V}}\right)} \cap \mathcal{E}_{\mathbf{S}}^{c} \cap \mathcal{E}_{\mathbf{X}}^{c} \cap \mathcal{E}_{\mathbf{Z}}^{c} \subseteq \mathcal{E}_{\left(\hat{\mathbf{U}}_{1}, \hat{\mathbf{U}}_{2}, \hat{\mathbf{V}}\right)}^{\prime} \cap \mathcal{E}_{\mathbf{S}}^{c} \cap \mathcal{E}_{\mathbf{X}}^{c} \cap \mathcal{E}_{\mathbf{Z}}^{c}
$$


and, in particular

$$
\operatorname{Pr}\left[\mathcal{E}_{\left(\hat{\mathbf{U}}_{1}, \hat{\mathbf{U}}_{2}, \hat{\mathbf{V}}\right)} \cap \mathcal{E}_{\mathbf{S}}^{c} \cap \mathcal{E}_{\mathbf{X}}^{c} \cap \mathcal{E}_{\mathbf{Z}}^{c}\right] \leq \operatorname{Pr}\left(\mathcal{E}_{\left(\hat{\mathbf{U}}_{1}, \hat{\mathbf{U}}_{2}, \hat{\mathbf{V}}\right)}^{\prime} \cap \mathcal{E}_{\mathbf{S}}^{c} \cap \mathcal{E}_{\mathbf{X}}^{c} \cap \mathcal{E}_{\mathbf{Z}}^{c}\right) .
$$

Proof: We first recall that for the event $\mathcal{E}_{\left(\hat{U}_{1}, \hat{U}_{2}, \hat{V}\right)}$ to occur, there must exist codewords $\mathbf{u}_{1}(j) \in \mathcal{C}_{1} \backslash\left\{\mathbf{u}_{1}^{*}\right\}$ and $\mathbf{u}_{2}(l) \in \mathcal{C}_{2} \backslash\left\{\mathbf{u}_{2}^{*}\right\}$ and $\mathbf{v}(k) \in \mathcal{C}_{c} \backslash\left\{\mathbf{v}^{*}\right\}$ such that the following inequalities are simultaneously satisfied

$$
\begin{aligned}
\left|\tilde{\rho}-\cos \varangle\left(\mathbf{u}_{1}(j), \mathbf{u}_{2}(l)\right)\right| & \leq 7 \epsilon \\
\left|\cos \varangle\left(\mathbf{v}(k), \mathbf{u}_{1}(j)\right)\right| & \leq 3 \epsilon \\
\left|\bar{\rho}-\cos \varangle\left(\mathbf{v}(k), \mathbf{u}_{2}(l)\right)\right| & \leq 7 \epsilon \\
\mid \mathbf{y}-\mathbf{X}_{\mathbf{u}_{1}(j), \mathbf{v}(k), \mathbf{u}_{2}(l)} \|^{2} & \leq\left\|\mathbf{y}-\mathbf{X}_{\mathbf{u}_{1}^{*}, \mathbf{v}^{*}, \mathbf{u}_{2}^{*}}\right\|^{2} .
\end{aligned}
$$

The proof is now based on a sequence of statements related to these conditions.

A) For every $\left(\mathbf{s}_{1}, \mathbf{s}_{2}, \mathcal{C}_{1}, \mathcal{C}_{2}, \mathcal{C}_{c}, \mathbf{z}\right) \in \mathcal{E}_{\mathbf{X}}^{c} \cap \mathcal{E}_{\mathbf{Z}}^{c}$,

$$
\begin{aligned}
& \mid \mathbf{y}-\mathbf{X}_{\mathbf{u}_{1}(j), \mathbf{v}(k), \mathbf{u}_{2}(l)}\left\|^{2} \leq\right\| \mathbf{y}-\mathbf{X}_{\mathbf{u}_{1}^{*}, \mathbf{v}^{*}, \mathbf{u}_{2}^{*}} \|^{2} \\
& \quad \Longrightarrow \\
& \quad\left\langle\mathbf{y}, a_{1} \mathbf{u}_{1}(j)+a_{2} \mathbf{u}_{2}(l)+\alpha \mathbf{v}(k)\right\rangle \\
& \quad \geq n\left(\bar{\beta}_{1} P_{1}+2 \sqrt{\bar{\beta}_{1} P_{1} \bar{\beta}_{2} P_{2}} \tilde{\rho}+\bar{\beta}_{2} P_{2}+2 \frac{1}{n}\left\|\alpha \mathbf{v}^{*}\right\| \sqrt{n \bar{\beta}_{2} P_{2}} \bar{\rho}+\frac{1}{n}\left\|\alpha \mathbf{v}^{*}\right\|^{2}-\xi_{1} \epsilon\right),
\end{aligned}
$$

where $\xi_{1}$ depends only on $P_{1}, P_{2}$ and $N$.

Statement A) follows by rewriting the 1.h.s. of (192) as

$$
\begin{aligned}
2\left\langle y, a_{1,1} \mathbf{u}_{1}(j)+a_{2,1} \mathbf{u}_{2}(l)+\alpha \mathbf{v}(k)\right\rangle \\
\geq 2\left\langle\mathbf{y}, a_{1,1} \mathbf{u}_{1}^{*}+a_{2,1} \mathbf{u}_{2}^{*}+\alpha \mathbf{v}^{*}\right\rangle+\left\|a_{1,1} \mathbf{u}_{1}(j)+a_{2,1} \mathbf{u}_{2}(l)+\alpha \mathbf{v}(k)\right\|^{2} \\
\quad-\left\|a_{1,1} \mathbf{u}_{1}^{*}+a_{2,1} \mathbf{u}_{2}^{*}+\alpha \mathbf{v}^{*}\right\|^{2} \\
=\left\|a_{1,1} \mathbf{u}_{1}^{*}+a_{2,1} \mathbf{u}_{2}^{*}+\alpha \mathbf{v}^{*}\right\|^{2}+2\left\langle\mathbf{z}, a_{1,1} \mathbf{u}_{1}^{*}+a_{2,1} \mathbf{u}_{2}^{*}+\alpha \mathbf{v}^{*}\right\rangle \\
\quad+\left\|a_{1,1} \mathbf{u}_{1}(j)+a_{2,1} \mathbf{u}_{2}(l)+\alpha \mathbf{v}(k)\right\|^{2} \\
\quad(a)) \\
\geq 2 n\left(\bar{\beta}_{1} P_{1}+2 \sqrt{\bar{\beta}_{1} P_{1} \bar{\beta}_{2} P_{2}} \tilde{\rho}+\bar{\beta}_{2} P_{2}+2 \frac{1}{n}\left\|\alpha \mathbf{v}^{*}\right\| \sqrt{n \bar{\beta}_{2} P_{2}} \bar{\rho}+\frac{1}{n}\left\|\alpha \mathbf{v}^{*}\right\|^{2}-\xi_{1} \epsilon\right),
\end{aligned}
$$

where in (a) we have used that $\left(\mathbf{s}_{1}, \mathbf{s}_{2}, \mathcal{C}_{1}, \mathcal{C}_{2}, \mathcal{C}_{c}, \mathbf{z}\right) \in \mathcal{E}_{\mathbf{X}}^{c} \cap \mathcal{E}_{\mathbf{Z}}^{c}$.

B) For every $\left(\mathbf{s}_{1}, \mathbf{s}_{2}, \mathcal{C}_{1}, \mathcal{C}_{2}, \mathcal{C}_{c}, \mathbf{z}\right) \in \mathcal{E}_{\mathbf{X}}^{c} \cap \mathcal{E}_{\mathbf{Z}}^{c}$,

$$
\|\mathbf{y}\|^{2} \leq n\left(\bar{\beta}_{1} P_{1}+2 \sqrt{\bar{\beta}_{1} P_{1} \bar{\beta}_{2} P_{2}} \tilde{\rho}+\bar{\beta}_{2} P_{2}+\frac{2}{n}\left\|\alpha \mathbf{v}^{*}\right\| \sqrt{n \bar{\beta}_{2} P_{2}} \bar{\rho}+\frac{1}{n}\left\|\alpha \mathbf{v}^{*}\right\|^{2}+N+\xi_{2} \epsilon\right),
$$

where $\xi_{2}$ depends only on $P_{1}, P_{2}$ and $N$.

Statement B) is obtained as follows:

$$
\begin{aligned}
& \|\mathbf{y}\|^{2}=\left\|a_{1,1} \mathbf{u}_{1}^{*}+a_{2,1} \mathbf{u}_{2}^{*}+\alpha \mathbf{v}^{*}+\mathbf{z}\right\|^{2} \\
& =\left\|a_{1,1} \mathbf{u}_{1}^{*}\right\|^{2}+2\left\langle a_{1,1} \mathbf{u}_{1}^{*}, a_{2,1} \mathbf{u}_{2}^{*}\right\rangle+\left\|a_{2,1} \mathbf{u}_{2}^{*}\right\|^{2}+2\left\langle a_{2,1} \mathbf{u}_{2}^{*}, \alpha \mathbf{v}^{*}\right\rangle+\left\|\alpha \mathbf{v}^{*}\right\|^{2} \\
& +2\left(\left\langle a_{1,1} \mathbf{u}_{1}^{*}, \mathbf{z}\right\rangle+\left\langle a_{2,1} \mathbf{u}_{2}^{*}, \mathbf{z}\right\rangle+\left\langle\alpha \mathbf{v}^{*}, \mathbf{z}\right\rangle\right)+\|\mathbf{z}\|^{2} \\
& \stackrel{(a)}{\leq} n \bar{\beta}_{1} P_{1}+2 n \sqrt{\bar{\beta}_{1} P_{1} \bar{\beta}_{2} P_{2}}(\tilde{\rho}+7 \epsilon)+\bar{\beta}_{2} P_{2}+2\left\|\alpha \mathbf{v}^{*}\right\| \sqrt{n \bar{\beta}_{2} P_{2}}(\bar{\rho}+7 \epsilon)+\left\|\alpha \mathbf{v}^{*}\right\|^{2} \\
& +2 n\left(\sqrt{\overline{\beta_{1}} P_{1} N} \epsilon+\sqrt{\bar{\beta}_{2} P_{2} N} \epsilon+\left\|\alpha \mathbf{v}^{*}\right\| \sqrt{n N} \epsilon\right)+n N(1+\epsilon)
\end{aligned}
$$




$$
=n\left(\bar{\beta}_{1} P_{1}+2 \sqrt{\bar{\beta}_{1} P_{1} \bar{\beta}_{2} P_{2}} \tilde{\rho}+\bar{\beta}_{2} P_{2}+\frac{2}{n}\left\|\alpha \mathbf{v}^{*}\right\| \sqrt{n \bar{\beta}_{2} P_{2}} \bar{\rho}+\frac{1}{n}\left\|\alpha \mathbf{v}^{*}\right\|^{2}+N+\xi_{2} \epsilon\right),
$$

where in (a) we have used that $\left(\mathbf{s}_{1}, \mathbf{s}_{2}, \mathcal{C}_{1}, \mathcal{C}_{2}, \mathcal{C}_{c}, \mathbf{z}\right) \in \mathcal{E}_{\mathbf{X}}^{c} \cap \mathcal{E}_{\mathbf{Z}}^{c}$.

C) For every $\left(\mathbf{s}_{1}, \mathbf{s}_{2}, \mathcal{C}_{1}, \mathcal{C}_{2}, \mathcal{C}_{c}, \mathbf{z}\right)$,

$$
\begin{aligned}
& \left|\tilde{\rho}-\left\langle\frac{\mathbf{u}_{1}(j)}{\left\|\mathbf{u}_{1}(j)\right\|}, \frac{\mathbf{u}_{2}(l)}{\left\|\mathbf{u}_{2}(l)\right\|}\right\rangle\right|<7 \epsilon \text { and }\left|\bar{\rho}-\left\langle\frac{\mathbf{u}_{2}(l)}{\left\|\mathbf{u}_{2}(l)\right\|}, \frac{\mathbf{v}(k)}{\|\mathbf{v}(k)\|}\right\rangle\right|<7 \epsilon \\
& \text { and }\left|\left\langle\frac{\mathbf{u}_{1}(j)}{\left\|\mathbf{u}_{1}(j)\right\|}, \frac{\mathbf{v}(k)}{\|\mathbf{v}(k)\|}\right\rangle\right|<3 \epsilon \\
& \quad \Longrightarrow\left\|a_{1,1} \mathbf{u}_{1}(j)+a_{2,1} \mathbf{u}_{2}(l)+\alpha \mathbf{v}(k)\right\|^{2} \\
& \quad \leq n\left(\bar{\beta}_{1} P_{1}+2 \sqrt{\bar{\beta}_{1} P_{1} \bar{\beta}_{2} P_{2}} \tilde{\rho}+\bar{\beta}_{2} P_{2}+2 \frac{1}{n}\left\|\alpha \mathbf{v}^{*}\right\| \sqrt{n \bar{\beta}_{2} P_{2}} \bar{\rho}+\frac{1}{n}\|\alpha \mathbf{v}(k)\|^{2}+\xi_{3} \epsilon\right) .
\end{aligned}
$$

Statement C) follows by

$$
\begin{aligned}
& \left\|a_{1,1} u_{1}(j)+a_{2,1} u_{2}(l)+\alpha \mathbf{v}(k)\right\|^{2} \\
& \quad=\left\|a_{1,1} u_{1}(j)\right\|^{2}+2\left\langle a_{1,1} \mathbf{u}_{1}(j), a_{2,1} \mathbf{u}_{2}(l)\right\rangle+\left\|a_{2,1} \mathbf{u}_{2}(l)\right\|^{2}+2\left\langle a_{2,1} \mathbf{u}_{2}(l), \alpha \mathbf{v}(k)\right\rangle+\|\alpha \mathbf{v}(k)\|^{2} \\
& \quad \stackrel{(a)}{\leq} n \bar{\beta}_{1} P_{1}+2 n \sqrt{\bar{\beta}_{1} P_{1} \bar{\beta}_{2} P_{2}}(\tilde{\rho}+7 \epsilon)+n \bar{\beta}_{2} P_{2}+2\left\|\alpha \mathbf{v}^{*}\right\| \sqrt{n \bar{\beta}_{2} P_{2}}(\bar{\rho}+7 \epsilon)+\|\alpha \mathbf{v}(k)\|^{2} \\
& \quad=n\left(\bar{\beta}_{1} P_{1}+2 \sqrt{\bar{\beta}_{1} P_{1} \bar{\beta}_{2} P_{2}} \tilde{\rho}+\bar{\beta}_{2} P_{2}+\frac{2}{n}\left\|\alpha \mathbf{v}^{*}\right\| \sqrt{n \bar{\beta}_{2} P_{2}} \bar{\rho}+\frac{1}{n}\|\alpha \mathbf{v}(k)\|^{2}+\xi_{3} \epsilon\right),
\end{aligned}
$$

where in (a) we have used that multiplying the first inequality on the 1.h.s. of (194) by $\left\|a_{1,1} \mathbf{u}_{1}(j)\right\| \cdot\left\|a_{2,1} \mathbf{u}_{2}(l)\right\|$ and recalling that $\left\|a_{1,1} \mathbf{u}_{1}(j)\right\| \leq \sqrt{n \bar{\beta}_{1} P_{1}}$ and that $\left\|a_{2,1} \mathbf{u}_{2}(l)\right\| \leq$ $\sqrt{n \bar{\beta}_{2} P_{2}}$ gives

$$
\left|n \tilde{\rho} \sqrt{\bar{\beta}_{1} P_{1} \bar{\beta}_{2} P_{2}}-\left\langle a_{1} \mathbf{u}_{1}(j), a_{2} \mathbf{u}_{2}(l)\right\rangle\right|<7 n \sqrt{\bar{\beta}_{1} P_{1} \bar{\beta}_{2} P_{2}} \epsilon
$$

and thus

$$
n \sqrt{\bar{\beta}_{1} P_{1} \bar{\beta}_{2} P_{2}}(\tilde{\rho}-7 \epsilon)<\left\langle a_{1,1} \mathbf{u}_{1}(j), a_{2,1} \mathbf{u}_{2}(l)\right\rangle<n \sqrt{\bar{\beta}_{1} P_{1} \bar{\beta}_{2} P_{2}}(\tilde{\rho}+7 \epsilon) .
$$

In a similar manner, we have used that multiplying the second inequality on the 1.h.s. of (194) by $\left\|a_{2,1} \mathbf{u}_{2}(l)\right\| \cdot\|\alpha \mathbf{v}(k)\|$ gives

$$
\left|\sqrt{n \bar{\beta}_{2} P_{2}} \bar{\rho}\|\alpha \mathbf{v}(k)\|-\left\langle a_{2} \mathbf{u}_{2}(l), \alpha \mathbf{v}(k)\right\rangle\right|<7 \sqrt{n \bar{\beta}_{2} P_{2}}\|\alpha \mathbf{v}(k)\| \epsilon,
$$

and thus

$$
\sqrt{n \overline{\beta_{2}} P_{2}}\|\alpha \mathbf{v}(k)\|(\bar{\rho}-7 \epsilon)<\left\langle a_{2} \mathbf{u}_{2}(l), \alpha \mathbf{v}(k)\right\rangle<\sqrt{n \overline{\beta_{2} P_{2}}}\|\alpha \mathbf{v}(k)\|(\bar{\rho}+7 \epsilon),
$$

thus establishing C).

D) For every $\left(\mathbf{s}_{1}, \mathbf{s}_{2}, \mathcal{C}_{1}, \mathcal{C}_{2}, \mathcal{C}_{c}, \mathbf{z}\right) \in \mathcal{E}_{\mathbf{X}}^{c} \cap \mathcal{E}_{\mathbf{Z}}^{c}$,

$$
\begin{aligned}
& \left(\left|\tilde{\rho}-\cos \varangle\left(\mathbf{u}_{1}(j), \mathbf{u}_{2}(l)\right)\right| \leq 7 \epsilon \quad \text { and } \quad\left|\cos \varangle\left(\mathbf{v}(k), \mathbf{u}_{1}(j)\right)\right| \leq 7 \epsilon\right. \\
& \text { and } \left.\quad\left|\bar{\rho}-\cos \varangle\left(\mathbf{v}(k), \mathbf{u}_{2}(l)\right)\right| \leq 7 \epsilon \quad \text { and } \quad \mid \mathbf{y}-\mathbf{X}_{\mathbf{u}_{1}(j), \mathbf{v}(k), \mathbf{u}_{2}(l)}\left\|^{2} \leq\right\| \mathbf{y}-\mathbf{X}_{\mathbf{u}_{1}^{*}, \mathbf{v}^{*}, \mathbf{u}_{2}^{*}} \|^{2}\right) \\
& \Longrightarrow \quad \cos \varangle\left(\mathbf{y}, a_{1,1} \mathbf{u}_{1}(j)+a_{2,1} \mathbf{u}_{2}(l)+\alpha \mathbf{v}(k)\right) \geq \Lambda(\epsilon) .
\end{aligned}
$$

Statement D) follows by rewriting $\cos \varangle\left(\mathbf{y}, a_{1,1} \mathbf{u}_{1}(j)+a_{2,1} \mathbf{u}_{2}(l)+\alpha \mathbf{v}(k)\right)$ as

$$
\cos \varangle\left(\mathbf{y}, a_{1,1} \mathbf{u}_{1}(j)+a_{2,1} \mathbf{u}_{2}(l)+\alpha \mathbf{v}(k)\right)=\frac{\left\langle\mathbf{y}, a_{1,1} \mathbf{u}_{1}(j)+a_{2,1} \mathbf{u}_{2}(l)+\alpha \mathbf{v}(k)\right\rangle}{\|\mathbf{y}\| \cdot\left\|a_{1,1} \mathbf{u}_{1}(j)+a_{2,1} \mathbf{u}_{2}(l)+\alpha \mathbf{v}(k)\right\|},
$$


and then lower bounding $\left\langle y, a_{1,1} \mathbf{u}_{1}(j)+a_{2,1} \mathbf{u}_{2}(l)+\alpha \mathbf{v}(k)\right\rangle$ using A), and upper-bounding $\|\mathbf{y}\|$ and $\left\|a_{1,1} \mathbf{u}_{1}(j)+a_{2,1} \mathbf{u}_{2}(l)+\alpha \mathbf{v}(k)\right\|$ using B) and C) respectively.

This yields that for every $\left(\mathbf{s}_{1}, \mathbf{s}_{2}, \mathcal{C}_{1}, \mathcal{C}_{2}, \mathcal{C}_{c}, \mathbf{z}\right) \in \mathcal{E}_{\mathbf{X}}^{c} \cap \mathcal{E}_{\mathbf{Z}}^{c}$,

$$
\begin{aligned}
\cos \varangle & \left(\mathbf{y}, a_{1,1} \mathbf{u}_{1}(j)+a_{2,1} \mathbf{u}_{2}(l)+\alpha \mathbf{v}(k)\right) \\
\geq & \frac{n\left(\bar{\beta}_{1} P_{1}+2 \sqrt{\bar{\beta}_{1} P_{1} \bar{\beta}_{2} P_{2}} \tilde{\rho}+\bar{\beta}_{2} P_{2}+2 \frac{1}{n}\left\|\alpha \mathbf{v}^{*}\right\| \sqrt{n \bar{\beta}_{2} P_{2}} \bar{\rho}+\frac{1}{n}\left\|\alpha \mathbf{v}^{*}\right\|^{2}-\xi_{1} \epsilon\right)}{\sqrt{n\left(\bar{\beta}_{1} P_{1}+2 \sqrt{\bar{\beta}_{1} P_{1} \bar{\beta}_{2} P_{2}} \tilde{\rho}+\bar{\beta}_{2} P_{2}+\frac{2}{n}\left\|\alpha \mathbf{v}^{*}\right\| \sqrt{n \bar{\beta}_{2} P_{2}} \bar{\rho}+\frac{1}{n}\left\|\alpha \mathbf{v}^{*}\right\|^{2}+N+\xi_{2} \epsilon\right)}} \\
& \cdot \frac{1}{\sqrt{n\left(\bar{\beta}_{1} P_{1}+2 \sqrt{\bar{\beta}_{1} P_{1} \bar{\beta}_{2} P_{2}} \tilde{\rho}+\bar{\beta}_{2} P_{2}+\frac{2}{n}\left\|\alpha \mathbf{v}^{*}\right\| \sqrt{n \bar{\beta}_{2} P_{2}} \bar{\rho}+\frac{1}{n}\|\alpha \mathbf{v}(k)\|^{2}+\xi_{3} \epsilon\right)}} \\
\geq & \sqrt{\frac{\bar{\beta}_{1} P_{1}+2 \sqrt{\bar{\beta}_{1} P_{1} \bar{\beta}_{2} P_{2}} \tilde{\rho}+\bar{\beta}_{2} P_{2}+2 \frac{1}{n}\left\|\alpha \mathbf{v}^{*}\right\| \sqrt{n \bar{\beta}_{2} P_{2}} \bar{\rho}+\frac{1}{n}\left\|\alpha \mathbf{v}^{*}\right\|^{2}-\xi^{\prime} \epsilon}{\bar{\beta}_{1}+2 \sqrt{\bar{\beta}_{1} P_{1} \bar{\beta}_{2} P_{2}} \tilde{\rho}+\bar{\beta}_{2} P_{2}+\frac{2}{n}\left\|\alpha \mathbf{v}^{*}\right\| \sqrt{n \bar{\beta}_{2} P_{2}} \bar{\rho}+\frac{1}{n}\left\|\alpha \mathbf{v}^{*}\right\|^{2}+N+\xi_{2} \epsilon}} \\
= & \Lambda(\epsilon) .
\end{aligned}
$$

Lemma 23 now follows by D) which gives

$$
\mathcal{E}_{\left(\hat{\mathbf{U}}_{1}, \hat{\mathbf{U}}_{2}, \hat{\mathbf{V}}\right)} \cap \mathcal{E}_{\mathbf{S}}^{c} \cap \mathcal{E}_{\mathbf{X}}^{c} \cap \mathcal{E}_{\mathbf{Z}}^{c} \subseteq \mathcal{E}_{\left(\hat{\mathbf{U}}_{1}, \hat{\mathbf{U}}_{2}, \hat{\mathbf{V}}\right)}^{\prime} \cap \mathcal{E}_{\mathbf{S}}^{c} \cap \mathcal{E}_{\mathbf{X}}^{c} \cap \mathcal{E}_{\mathbf{Z}}^{c},
$$

and therefore

$$
\operatorname{Pr}\left[\mathcal{E}_{\left(\hat{\mathbf{U}}_{1}, \hat{\mathbf{U}}_{2}, \hat{\mathbf{V}}\right)} \mid \epsilon_{S}^{c} \cap \mathcal{E}_{\mathbf{X}}^{c} \cap \mathcal{E}_{\mathbf{Z}}^{c}\right] \leq \operatorname{Pr}\left[\mathcal{E}_{\left(\hat{\mathbf{U}}_{1}, \hat{\mathbf{U}}_{2}, \hat{\mathbf{V}}\right)}^{\prime} \mid \mathcal{E}_{\mathbf{S}}^{c} \cap \mathcal{E}_{\mathbf{X}}^{c} \cap \mathcal{E}_{\mathbf{Z}}^{c}\right]
$$

We now state the second lemma needed for the proof of (108).

Lemma 24 For every $\Theta_{i} \in(0,1], i=1,2$ and $\Delta \in(0,1]$, let the set $\mathcal{G}$ be given by

$$
\begin{gathered}
\mathcal{G}=\left\{\left(\mathbf{s}_{1}, \mathbf{s}_{2}, \mathcal{C}_{1}, \mathcal{C}_{2}, \mathcal{C}_{c}, \mathbf{z}\right): \exists \mathbf{u}_{1}(j) \in \mathcal{C}_{1} \backslash\left\{\mathbf{u}_{1}^{*}\right\} \text { and } \exists \mathbf{u}_{2}(l) \in \mathcal{C}_{2} \backslash\left\{\mathbf{u}_{2}^{*}\right\}\right. \\
\quad \text { and } \exists \mathbf{v}(k) \in \mathcal{C}_{c} \backslash\left\{\mathbf{v}^{*}\right\} \text { s.t. } \cos \varangle\left(\mathbf{u}_{1}(j), \mathbf{u}_{2}(l)\right) \geq \Theta_{1}, \cos \varangle\left(\mathbf{u}_{2}(l), \mathbf{v}(l)\right) \geq \Theta_{2}, \\
\text { and } \left.\cos \varangle\left(\mathbf{y}, a_{1} \mathbf{u}_{1}(j)+a_{2} \mathbf{u}_{2}(l)+\alpha \mathbf{v}(k)\right) \geq \Delta\right\} .
\end{gathered}
$$

Then,

$$
\begin{array}{r}
R_{1}+R_{2}+R_{\mathrm{c}}<-\frac{1}{2} \log \left(\left(1-\Theta_{1}{ }^{2}\right)\left(1-\Theta_{2}{ }^{2}\right)\left(1-\Delta^{2}\right)\right) \\
\Longrightarrow\left(\lim _{n \rightarrow \infty} \operatorname{Pr}\left[\mathcal{G} \mid \mathcal{E}_{\mathbf{X}_{1}}^{c} \cap \mathcal{E}_{\mathbf{X}_{2}}^{c} \cap \mathcal{E}_{\mathbf{X}_{V}}^{c}\right]=0, \epsilon>0\right) .
\end{array}
$$

Proof: The proof follows from upper-bounding in every point on $\mathcal{S}_{i}, i \in 1,2$ and $\mathcal{S}_{c}$, the density of every $\mathbf{u}_{i}(j) \in \mathcal{C}_{i} \backslash\left\{\mathbf{u}_{i}^{*}\right\}$ and every $\mathbf{v}(k) \in \mathcal{C}_{c} \backslash\left\{\mathbf{v}^{*}\right\}$ and then using a standard argument from sphere-packing. This follows similarly as the proof of lemma D.9 in [1], using Lemma 27 ahead.

Now we can turn to the proof of (108).

$$
\begin{aligned}
\operatorname{Pr}\left[\mathcal{E}_{\left(\hat{\mathbf{U}}_{1}, \hat{\mathbf{U}}_{2}, \hat{\mathbf{V}}\right)} \cap \mathcal{E}_{\mathbf{S}}^{c} \cap \mathcal{E}_{\mathbf{X}}^{c} \cap \mathcal{E}_{\mathbf{Z}}^{c}\right] & \stackrel{(a)}{\leq} \operatorname{Pr}\left[\mathcal{E}_{\left(\hat{\mathbf{U}}_{1}, \hat{\mathbf{U}}_{2}, \hat{\mathbf{V}}\right)}^{\prime} \cap \mathcal{E}_{\mathbf{S}}^{c} \cap \mathcal{E}_{\mathbf{X}}^{c} \cap \mathcal{E}_{\mathbf{Z}}^{c}\right] \\
& \stackrel{(b)}{\leq} \operatorname{Pr}\left[\mathcal{E}_{\left(\hat{\mathbf{U}}_{1}, \hat{\mathbf{U}}_{2}, \hat{\mathbf{V}}\right)}^{\prime} \mid \mathcal{E}_{\mathbf{X}_{1}}^{c} \cap \mathcal{E}_{\mathbf{X}_{2}}^{c} \cap \mathcal{E}_{X v}^{c}\right],
\end{aligned}
$$

where (a) follows by Lemma 23 and (b) follows because $\mathcal{E}_{\mathbf{X}}^{c} \subseteq\left(\mathcal{E}_{\mathbf{X}_{1}}^{c} \cap \mathcal{E}_{\mathbf{X}_{2}}^{c} \cap \mathcal{E}_{X v}^{c}\right)$. 
The proof of (108) is now completed by combining Inequality (195) with Lemma 24, which gives that for every $\delta>0$ and every $\epsilon>0$, there exists some $n^{\prime}(\delta, \epsilon)$ such that for all $n>n^{\prime}(\delta, \epsilon)$, we have

whenever

$$
\operatorname{Pr}\left[\mathcal{E}_{\left(\hat{\mathbf{U}}_{1}, \hat{\mathbf{U}}_{2}, \hat{\mathbf{V}}\right)} \cap \mathcal{E}_{\mathbf{S}}^{c} \cap \mathcal{E}_{\mathbf{X}}^{c} \cap \mathcal{E}_{\mathbf{Z}}^{c}\right] \leq \operatorname{Pr}\left[\mathcal{E}_{\left(\hat{\mathbf{U}}_{1}, \hat{\mathbf{U}}_{2}, \hat{\mathbf{V}}\right)}^{\prime} \mid \mathcal{E}_{\mathbf{X}_{1}}^{c} \cap \mathcal{E}_{\mathbf{X}_{2}}^{c}\right]<\delta,
$$

$$
R_{1}+R_{2}+R_{\mathrm{c}}<\frac{1}{2} \log \left(\frac{\lambda_{12}+2 \eta \bar{\rho} \sqrt{\bar{\beta}_{2} P_{2}}+\eta^{2}+N}{N\left(1-\tilde{\rho}^{2}\right)\left(1-\bar{\rho}^{2}\right)}-\kappa_{7} \epsilon\right),
$$

where $\kappa_{7}$ is is a positive constant determined by $P_{1}, P_{2}$ and $N$.

The proof of Lemma 9 is now completed.

The proof of Lemma 5 now follows straight forwardly:

Proof of Lemma 5: Combining (101) with Lemma 6, Lemma 7 Lemma 8 and Lemma 9 yields that for every $\delta>0$ and $0<\epsilon<0.3$, there exists an $n^{\prime}(\delta, \epsilon) \in \mathbb{N}$ such that for all $n>n^{\prime}(\delta, \epsilon)$

$$
\operatorname{Pr}\left[\mathcal{E}_{\hat{\mathbf{U}}}\right]<21 \delta, \quad \text { if }\left(R_{1}, R_{2}, R_{\mathrm{c}}\right) \in \mathcal{R}(\epsilon)
$$

\section{H. Proof of Lemma 8}

The proofs in this section rely on bounds from the geometry of sphere packing. To this end, we denote by $C_{n}(\varphi)$ the surface area of a polar cap of half angle $\varphi$ on an $\mathbb{R}^{n}$-sphere of unit radius. Upper and lower bounds on the surface area $C_{n}(\varphi)$ are given in the following lemma.

Lemma 25 For any $\varphi \in[0, \pi / 2]$,

$$
\frac{\Gamma\left(\frac{n}{2}+1\right) \sin ^{(n-1)} \varphi}{n \Gamma\left(\frac{n+1}{2}\right) \sqrt{\pi} \cos \varphi}\left(1-\frac{1}{n} \tan ^{2} \varphi\right) \leq \frac{C_{n}(\varphi)}{C_{n}(\pi)} \leq \frac{\Gamma\left(\frac{n}{2}+1\right) \sin ^{(n-1)} \varphi}{n \Gamma\left(\frac{n+1}{2}\right) \sqrt{\pi} \cos \varphi} .
$$

Proof: See [21, Inequality (27)].

The ratio of the two gamma functions that appears in the upper bound and the lower bound of Lemma 25 has the following asymptotic series.

\section{Lemma 26}

$$
\frac{\Gamma\left(x+\frac{1}{2}\right)}{\Gamma(x)}=\sqrt{x}\left(1-\frac{1}{8 x}+\frac{1}{128 x^{2}}+\frac{5}{1024 x^{3}}-\frac{21}{32678 x^{4}}+\ldots\right),
$$

and in particular

$$
\lim _{x \rightarrow \infty} \frac{\Gamma\left(x+\frac{1}{2}\right)}{\Gamma(x)}=1
$$

\section{Proof: See [1, Appendix D-E].}

Before starting with the proofs of this section, we give one more lemma. To this end, whenever the vector-quantizer of Encoder 1 does not produce the all-zero sequence, denote by $\varsigma_{1}\left(\mathbf{s}_{1}, \mathcal{C}_{1}\right)$ the index of $\mathbf{u}_{1}^{*}$ in its codebook $\mathcal{C}_{1}$. And whenever the vector-quantizer of Encoder 1 produces the all-zero sequence, let $\varsigma_{1}\left(\mathbf{s}_{1}, \mathcal{C}_{1}\right)=0$. Further, let $\lambda_{1}(\cdot)$ denote the measure on the codeword sphere $\mathcal{S}_{1}$ induced by the uniform distribution, and let $f^{\lambda_{1}}(\cdot)$ denote the density on $\mathcal{S}_{1}$ with respect to $\lambda_{1}(\cdot)$. Similarly, for Encoder 2 define $\varsigma_{2}\left(\mathbf{s}_{2}, \mathcal{C}_{2}\right)$ and $f^{\lambda_{2}}(\cdot)$ accordingly. 
Lemma 27 Conditioned on $\varsigma_{1}\left(\mathbf{s}_{1}, \mathscr{C}_{1}\right)=1$, the density of $\mathbf{U}_{1}(j)$ is upper bounded for every $j \in\left\{2,3, \ldots, 2^{n R_{1}}\right\}$ and at every point $\mathbf{u} \in \mathcal{S}_{1}$ by twice the uniform density:

$$
f^{\lambda_{1}}\left(\mathbf{U}_{1}(j)=\mathbf{u} \mid \varsigma_{1}\left(\mathbf{s}_{1}, \mathcal{C}_{1}\right)=1\right) \leq 2 \cdot \frac{1}{r_{1}^{n-1} C_{n}(\pi)},
$$

and similarly for Encoder 2.

\section{Proof: See [1, Appendix D-E]}

\section{Proof of Lemma 8}

We begin with the following decomposition

$$
\begin{aligned}
& \operatorname{Pr}\left[\mathcal{E}_{\mathbf{X}}\right]=\operatorname{Pr}\left[\mathcal{E}_{\mathbf{X}} \cap \mathcal{E}_{\mathbf{S}}\right]+\operatorname{Pr}\left[\mathcal{E}_{\mathbf{X}} \cap \mathcal{E}_{\mathbf{S}}^{c}\right] \\
& \leq \operatorname{Pr}\left[\mathcal{E}_{\mathbf{S}}\right]+\operatorname{Pr}\left[\mathcal{E}_{\mathbf{X}_{1}} \cap \mathcal{E}_{\mathbf{S}}^{c}\right]+\operatorname{Pr}\left[\mathcal{E}_{\mathbf{X}_{2}} \cap \mathcal{E}_{\mathbf{S}}^{c}\right]+\operatorname{Pr}\left[\mathcal{E}_{\mathbf{X}_{v}} \cap \mathcal{E}_{\mathbf{S}}^{c}\right]+\operatorname{Pr}\left[\mathcal{E}_{\left(\mathbf{X}_{1}, \mathbf{X}_{2}\right)} \cap \mathcal{E}_{\mathbf{X}_{1}}^{c} \cap \mathcal{E}_{\mathbf{X}_{2}}^{c} \cap \mathcal{E}_{\mathbf{S}}^{c}\right] \\
& +\operatorname{Pr}\left[\mathcal{E}_{\left(\mathbf{X}_{1}, \mathbf{X}_{v}\right)} \cap \mathcal{E}_{\mathbf{X}_{1}}^{c} \cap \mathcal{E}_{\mathbf{X}_{v}}^{c} \cap \mathcal{E}_{\mathbf{S}}^{c}\right]+\operatorname{Pr}\left[\mathcal{E}_{\left(\mathbf{X}_{2}, \mathbf{X}_{v}\right)} \cap \mathcal{E}_{\mathbf{X}_{2}}^{c} \cap \mathcal{E}_{\mathbf{X}_{v}}^{c} \cap \mathcal{E}_{\mathbf{S}}^{c}\right]+\operatorname{Pr}\left[\mathcal{E}_{\mathbf{X}_{\mathrm{WZ}}} \cap \mathcal{E}_{\mathbf{X}_{v}}^{c} \cap \mathcal{E}_{\mathbf{S}}^{c}\right] \\
& \leq \operatorname{Pr}\left[\mathcal{E}_{\mathbf{S}}\right]+\operatorname{Pr}\left[\mathcal{E}_{\mathbf{X}_{1}}\right]+\operatorname{Pr}\left[\mathcal{E}_{\mathbf{X}_{2}}\right]+\operatorname{Pr}\left[\mathcal{E}_{\mathbf{X}_{v}}\right]+\operatorname{Pr}\left[\mathcal{E}_{\left(\mathbf{X}_{1}, \mathbf{X}_{2}\right)} \cap \mathcal{E}_{\mathbf{X}_{1}}^{c} \cap \mathcal{E}_{\mathbf{X}_{2}}^{c} \cap \mathcal{E}_{\mathbf{S}}^{c}\right] \\
& +\operatorname{Pr}\left[\mathcal{E}_{\left(\mathbf{X}_{1}, \mathbf{X}_{v}\right)} \cap \mathcal{E}_{\mathbf{X}_{1}}^{c} \cap \mathcal{E}_{\mathbf{X}_{v}}^{c} \cap \mathcal{E}_{\mathbf{S}}^{c}\right]+\operatorname{Pr}\left[\mathcal{E}_{\left(\mathbf{X}_{2}, \mathbf{X}_{v}\right)} \cap \mathcal{E}_{\mathbf{X}_{2}}^{c} \cap \mathcal{E}_{\mathbf{X}_{v}}^{c} \cap \mathcal{E}_{\mathbf{S}}^{c}\right]+\operatorname{Pr}\left[\mathcal{E}_{\mathbf{X}_{\mathrm{wZ}}} \cap \mathcal{E}_{\mathbf{X}_{v}}^{c} \cap \mathcal{E}_{\mathbf{S}}^{c}\right] .
\end{aligned}
$$

The proof of Lemma 8 now follows by showing that for every $\delta>0$ and $0.3>\epsilon>0$ there exists an $n_{2}^{\prime}(\delta, \epsilon)>0$ such that for all $n>n_{2}^{\prime}(\delta, \epsilon)>0$

$$
\begin{aligned}
& \operatorname{Pr}\left[\mathcal{E}_{\mathbf{X}_{i}}\right] \leq \delta, \quad i \in\{1,2, v\} \\
& \operatorname{Pr}\left[\mathcal{E}_{\left(\mathbf{X}_{1}, \mathbf{X}_{2}\right)} \cap \mathcal{E}_{\mathbf{X}_{1}}^{c} \cap \mathcal{E}_{\mathbf{X}_{2}}^{c} \cap \mathcal{E}_{\mathbf{S}}^{c}\right] \leq 3 \delta, \\
& \operatorname{Pr}\left[\mathcal{E}_{\left(\mathbf{X}_{1}, \mathbf{X}_{v}\right)} \cap \mathcal{E}_{\mathbf{X}_{1}}^{c} \cap \mathcal{E}_{\mathbf{X}_{v}}^{c} \cap \mathcal{E}_{\mathbf{S}}^{c}\right] \leq \delta, \\
& \operatorname{Pr}\left[\mathcal{E}_{\left(\mathbf{X}_{2}, \mathbf{X}_{v}\right)} \cap \mathcal{E}_{\mathbf{X}_{2}}^{c} \cap \mathcal{E}_{\mathbf{X}_{v}}^{c} \cap \mathcal{E}_{\mathbf{S}}^{c}\right] \leq 3 \delta, \\
& \operatorname{Pr}\left[\mathcal{E}_{\mathbf{X}_{\mathrm{WZ}}} \cap \mathcal{E}_{\mathbf{X}_{v}}^{c} \cap \mathcal{E}_{\mathbf{S}}^{c}\right] \leq \delta .
\end{aligned}
$$

Proof of [196): We give the proof for $\mathcal{E}_{\mathbf{X}_{1}}$. Due to the symmetry the proof for $\mathcal{E}_{\mathbf{X}_{2}}$ and $\mathcal{E}_{\mathbf{V}}$ then follows by similar arguments. Let $\mathcal{E}_{\mathbf{X}_{1}}(j)$ be the event that $\mathbf{U}_{1}(j)$ does not have a typical angle to $\mathbf{S}_{1}$, i.e.

$$
\mathcal{E}_{\mathbf{X}_{1}}(j)=\left\{\left(\mathbf{s}_{1}, \mathbf{s}_{2}, \mathcal{C}_{1}, \mathcal{C}_{2}, \mathcal{C}_{c}\right):\left|\cos \varangle\left(\mathbf{u}_{1}(j), \mathbf{s}_{1}\right)-\sqrt{1-2^{-2 R_{1}}}\right|>\epsilon \sqrt{1-2^{-2 R_{1}}}\right\} .
$$

Then,

$$
\begin{aligned}
\operatorname{Pr}\left[\mathcal{E}_{\mathbf{X}_{1}}\right] & =\operatorname{Pr}\left[\mathcal{E}_{\mathbf{X}_{1}} \mid \mathbf{S}_{1}=\mathbf{s}_{1}\right] \\
& =\operatorname{Pr}\left[\bigcap_{j=1}^{2^{n R_{1}}} \mathcal{E}_{\mathbf{X}_{1}}(j) \mid \mathbf{S}_{1}=\mathbf{s}_{1}\right] \\
& =\prod_{j=1}^{2^{n R_{1}}} \operatorname{Pr}\left[\mathcal{E}_{\mathbf{X}_{1}}(j) \mid \mathbf{S}_{1}=\mathbf{s}_{1}\right] \\
& \stackrel{(a)}{=} \prod_{j=1}^{2^{n R_{1}}} \operatorname{Pr}\left[\mathcal{E}_{\mathbf{X}_{1}}(j)\right] \\
& \stackrel{(b)}{=}\left(\operatorname{Pr}\left[\mathcal{E}_{\mathbf{X}_{1}}(1)\right]\right)^{2^{n R_{1}}} \\
& =\left(1-\operatorname{Pr}\left[\mathcal{E}_{\mathbf{X}_{1}}^{c}(1)\right]\right)^{2^{n R_{1}}},
\end{aligned}
$$

where in (a) we have used that the probability of $\mathcal{E}_{\mathbf{X}_{1}}(j)$ does not depend on $\mathbf{S}_{1}=\mathbf{s}_{1}$, and in (b) we have used that all $\mathbf{U}_{1}(j)$ have the same distribution. To upper-bound 201) we now rewrite 
$\mathcal{E}_{\mathbf{X}_{1}}^{c}(1)$ as

$$
\begin{aligned}
& \mathcal{E}_{\mathbf{X}_{1}}^{c}(1) \\
& =\left\{\left(\mathbf{s}_{1}, \mathbf{s}_{2}, \mathcal{C}_{1}, \mathcal{C}_{2}, \mathcal{C}_{c}\right):\left|\cos \varangle\left(\mathbf{u}_{1}(1), \mathbf{s}_{1}\right)-\sqrt{1-2^{-2 R_{1}}}\right| \leq \epsilon \sqrt{1-2^{-2 R_{1}}}\right\} \\
& =\left\{\left(\mathbf{s}_{1}, \mathbf{s}_{2}, \mathcal{C}_{1}, \mathcal{C}_{2}, \mathcal{C}_{c}\right): \quad \sqrt{1-2^{-2 R_{1}}}(1-\epsilon) \leq \cos \varangle\left(\mathbf{u}_{1}(1), \mathbf{s}_{1}\right) \leq \sqrt{1-2^{-2 R_{1}}}(1+\epsilon)\right\} \\
& =\left\{\left(\mathbf{s}_{1}, \mathbf{s}_{2}, \mathcal{C}_{1}, \mathcal{C}_{2}, \mathcal{C}_{c}\right): \quad \cos \theta_{1, \max } \leq \cos \varangle\left(\mathbf{u}_{1}(1), \mathbf{s}_{1}\right) \leq \cos \theta_{1, \min }\right\},
\end{aligned}
$$

where we have used the notation

$$
\begin{aligned}
& \cos \theta_{1, \max } \triangleq \sqrt{1-2^{-2 R_{1}}}(1-\epsilon) \\
& \cos \theta_{1, \text { min }} \triangleq \sqrt{1-2^{-2 R_{1}}}(1+\epsilon) .
\end{aligned}
$$

Hence, since $\mathbf{U}_{1}(1)$ is generated independently of $\mathbf{S}_{1}$ and distributed uniformly on $\mathcal{S}_{1}$,

$$
\operatorname{Pr}\left[\mathcal{E}_{\mathbf{X}_{1}}^{c}(1)\right]=\frac{C_{n}\left(\theta_{1, \max }\right)-C_{n}\left(\theta_{1, \min }\right)}{C_{n}(\pi)} .
$$

Combining (202) with (201) gives, as reported in [1, Appendix D-E1],

$$
\begin{gathered}
\operatorname{Pr}\left[\mathcal{E}_{\mathbf{X}_{1}}\right] \leq \exp \left(-\frac{\Gamma\left(\frac{n}{2}+1\right)}{n \Gamma\left(\frac{n+1}{2}\right) \sqrt{\pi}}\left[\frac{2^{n\left(R_{1}+\log _{2}\left(\sin \theta_{1, \max }\right)\right)}}{\sin \theta_{1, \max } \cos \theta_{1, \max }}\left(1-\frac{1}{n} \tan ^{2} \theta_{1, \max }\right)\right.\right. \\
\left.\left.-\frac{2^{n\left(R_{1}+\log _{2}\left(\sin \theta_{1, \min }\right)\right)}}{\sin \theta_{1, \min } \cos \theta_{1, \min }}\right]\right) .
\end{gathered}
$$

It now follows from sphere-packing and covering, that for every $\epsilon>0$ we have $\operatorname{Pr}\left[\epsilon_{x_{1}}\right] \rightarrow 0$ as $n \rightarrow \infty$, as reported in [1, Appendix D-E1].

Proof of inequality (197): By the notation in 236) we have

$$
\begin{aligned}
\cos \varangle\left(\mathbf{u}_{1}^{*}, \mathbf{u}_{2}^{*}\right) & =\frac{\left\langle\mathbf{u}_{1}^{*}, \mathbf{u}_{2}^{*}\right\rangle}{\left\|\mathbf{u}_{1}^{*}\right\|\left\|\mathbf{u}_{2}^{*}\right\|} \\
& =\frac{\left\langle\nu_{1} \mathbf{s}_{1}+\mathbf{w}_{1}, \nu_{2} \mathbf{s}_{2}+\mathbf{w}_{2}\right\rangle}{\left\|\mathbf{u}_{1}^{*}\right\|\left\|\mathbf{u}_{2}^{*}\right\|} \\
& =\frac{\nu_{1} \nu_{2}\left\langle\mathbf{s}_{1}, \mathbf{s}_{2}\right\rangle+\nu_{1}\left\langle\mathbf{s}_{1}, \mathbf{w}_{2}\right\rangle+\nu_{2}\left\langle\mathbf{w}_{1}, \mathbf{s}_{2}\right\rangle+\left\langle\mathbf{w}_{1}, \mathbf{w}_{2}\right\rangle}{\left\|\mathbf{u}_{1}^{*}\right\|\left\|\mathbf{u}_{2}^{*}\right\|},
\end{aligned}
$$

where we recall that $\nu_{1}$ is a function of $\left\|\mathbf{s}_{1}\right\|$ and $\cos \varangle\left(\mathbf{s}_{1}, \mathbf{u}_{1}^{*}\right)$ and similarly $\nu_{2}$ is a function of $\left\|\mathbf{s}_{2}\right\|$ and $\cos \varangle\left(\mathbf{s}_{2}, \mathbf{u}_{2}^{*}\right)$. Now, define the four events

$$
\begin{aligned}
& \mathcal{A}_{1}=\left\{\left(\mathbf{s}_{1}, \mathbf{s}_{2}, \mathcal{C}_{1}, \mathcal{C}_{2}, \mathcal{C}_{c}\right):\left|\tilde{\rho}-\frac{\nu_{1} \nu_{2}}{\left\|\mathbf{u}_{1}^{*}\right\|\left\|\mathbf{u}_{2}^{*}\right\|}\left\langle\mathbf{s}_{1}, \mathbf{s}_{2}\right\rangle\right|>4 \epsilon\right\} \\
& \mathcal{A}_{2}=\left\{\left(\mathbf{s}_{1}, \mathbf{s}_{2}, \mathcal{C}_{1}, \mathcal{C}_{2}, \mathcal{C}_{c}\right):\left|\frac{\nu_{1}}{\left\|\mathbf{u}_{1}^{*}\right\|\left\|\mathbf{u}_{2}^{*}\right\|}\left\langle\mathbf{s}_{1}, \mathbf{w}_{2}\right\rangle\right|>\epsilon\right\} \\
& \mathcal{A}_{3}=\left\{\left(\mathbf{s}_{1}, \mathbf{s}_{2}, \mathcal{C}_{1}, \mathcal{C}_{2}, \mathcal{C}_{c}\right):\left|\frac{\nu_{2}}{\left\|\mathbf{u}_{1}^{*}\right\|\left\|\mathbf{u}_{2}^{*}\right\|}\left\langle\mathbf{w}_{1}, \mathbf{s}_{2}\right\rangle\right|>\epsilon\right\} \\
& \mathcal{A}_{4}=\left\{\left(\mathbf{s}_{1}, \mathbf{s}_{2}, \mathcal{C}_{1}, \mathcal{C}_{2}, \mathcal{C}_{c}\right):\left|\frac{1}{\left\|\mathbf{u}_{1}^{*}\right\|\left\|\mathbf{u}_{2}^{*}\right\|}\left\langle\mathbf{w}_{1}, \mathbf{w}_{2}\right\rangle\right|>\epsilon\right\} .
\end{aligned}
$$

Note that by (204),

$$
\begin{gathered}
\mathcal{E}_{\left(\mathbf{X}_{1}, \mathbf{X}_{2}\right)}=\left\{\left(\mathbf{s}_{1}, \mathbf{s}_{2}, \mathcal{C}_{1}, \mathcal{C}_{2}, \mathcal{C}_{c}\right):\left|\tilde{\rho}-\cos \varangle\left(\mathbf{u}_{1}^{*}, \mathbf{u}_{2}^{*}\right)\right|>7 \epsilon\right\} \subset\left(\mathcal{A}_{1} \cup \mathcal{A}_{2} \cup \mathcal{A}_{3} \cup \mathcal{A}_{4}\right) . \text { Thus, } \\
\operatorname{Pr}\left[\mathcal{E}_{\left(\mathbf{X}_{1}, \mathbf{X}_{2}\right)} \cap \mathcal{E}_{\mathbf{X}_{1}}^{c} \cap \mathcal{E}_{\mathbf{X}_{2}}^{c} \cap \mathcal{E}_{\mathbf{S}}^{c}\right] \\
\leq \operatorname{Pr}\left[\mathcal{A}_{1} \cap \mathcal{E}_{\mathbf{X}_{1}}^{c} \cap \mathcal{E}_{\mathbf{X}_{2}}^{c} \cap \mathcal{E}_{\mathbf{S}}^{c}\right]+\operatorname{Pr}\left[\mathcal{A}_{2} \cap \mathcal{E}_{\mathbf{X}_{1}}^{c} \cap \mathcal{E}_{\mathbf{X}_{2}}^{c} \cap \mathcal{E}_{\mathbf{S}}^{c}\right]
\end{gathered}
$$




$$
\begin{aligned}
& +\operatorname{Pr}\left[\mathcal{A}_{3} \cap \mathcal{E}_{\mathbf{X}_{1}}^{c} \cap \mathcal{E}_{\mathbf{X}_{2}}^{c} \cap \mathcal{E}_{\mathbf{S}}^{c}\right]+\operatorname{Pr}\left[\mathcal{A}_{4} \cap \mathcal{E}_{\mathbf{X}_{1}}^{c} \cap \mathcal{E}_{\mathbf{X}_{2}}^{c} \cap \mathcal{E}_{\mathbf{S}}^{c}\right] \\
\leq & \operatorname{Pr}\left[\mathcal{A}_{1} \mid \mathcal{E}_{\mathbf{X}_{1}}^{c} \cap \mathcal{E}_{\mathbf{X}_{2}}^{c} \cap \mathcal{E}_{\mathbf{S}}^{c}\right]+\operatorname{Pr}\left[\mathcal{A}_{2} \mid \mathcal{E}_{\mathbf{S}}^{c}\right]+\operatorname{Pr}\left[\mathcal{A}_{3} \mid \mathcal{E}_{\mathbf{S}}^{c}\right]+\operatorname{Pr}\left[\mathcal{A}_{4} \mid \mathcal{E}_{\mathbf{S}}^{c}\right]
\end{aligned}
$$

The four terms on the r.h.s. of (205) are now bounded in the following two lemmas.

Lemma 28 For $\epsilon<0.3$

$$
\operatorname{Pr}\left[\mathcal{A}_{1} \mid \mathcal{E}_{\mathbf{S}}^{c} \cap \mathcal{E}_{\mathbf{X}_{1}}^{c} \cap \mathcal{E}_{\mathbf{X}_{2}}^{c}\right]=0 .
$$

Proof: We first note that the term in the definition of $\mathcal{A}_{1}$ can be rewritten as

$$
\frac{\nu_{1} \nu_{2}}{\left\|\mathbf{u}_{1}^{*}\right\|\left\|\mathbf{u}_{2}^{*}\right\|}\left\langle\mathbf{s}_{1}, \mathbf{s}_{2}\right\rangle=\cos \varangle\left(\mathbf{s}_{1}, \mathbf{u}_{1}^{*}\right) \cos \varangle\left(\mathbf{s}_{2}, \mathbf{u}_{1}^{*}\right) \cos \varangle\left(\mathbf{s}_{1}, \mathbf{s}_{2}\right) .
$$

We can now upper and lower bound the r.h.s. of (206) for $\left(\mathbf{s}_{1}, \mathbf{s}_{2}, \mathcal{C}_{1}, \mathcal{C}_{2}, \mathcal{C}_{c}\right) \in \mathcal{E}_{\mathbf{S}}^{c} \cap \mathcal{E}_{\mathbf{X}_{1}}^{c} \cap \mathcal{E}_{\mathbf{X}_{2}}^{c}$ by noticing that $\left(\mathbf{s}_{1}, \mathbf{s}_{2}, \mathcal{C}_{1}, \mathcal{C}_{2}, \mathcal{C}_{c}\right) \in \mathcal{E}_{\mathbf{S}}^{c}$ implies

$$
\left|\cos \varangle\left(\mathbf{s}_{1}, \mathbf{s}_{2}\right)-\rho\right|<\rho \epsilon,
$$

that $\left(\mathbf{s}_{1}, \mathbf{s}_{2}, \mathcal{C}_{1}, \mathcal{C}_{2}, \mathcal{C}_{c}\right) \in \mathcal{E}_{\mathbf{X}_{1}}^{c}$ implies

$$
\left|\sqrt{1-2^{-2 R_{1}}}-\cos \varangle\left(\mathbf{s}_{1}, \mathbf{u}_{1}^{*}\right)\right|<\epsilon \sqrt{1-2^{-2 R_{1}}},
$$

and that $\left(\mathbf{s}_{1}, \mathbf{s}_{2}, \mathcal{C}_{1}, \mathcal{C}_{2}, \mathcal{C}_{c}\right) \in \mathcal{E}_{\mathbf{X}_{2}}^{c}$ implies

$$
\left|\sqrt{1-2^{-2 R_{2}}}-\cos \varangle\left(\mathbf{s}_{2}, \mathbf{u}_{2}^{*}\right)\right|<\epsilon \sqrt{1-2^{-2 R_{2}}} .
$$

Hence, combined with (206) this gives

$$
\tilde{\rho}(1-\epsilon)^{3} \leq \frac{\nu_{1} \nu_{2}}{\left\|\mathbf{u}_{1}^{*}\right\|\left\|\mathbf{u}_{2}^{*}\right\|}\left\langle\mathbf{s}_{1}, \mathbf{s}_{2}\right\rangle \leq \tilde{\rho}(1+\epsilon)^{3},
$$

whenever $\left(\mathbf{s}_{1}, \mathbf{s}_{2}, \mathcal{C}_{1}, \mathcal{C}_{2}, \mathcal{C}_{c}\right) \in \mathcal{E}_{\mathbf{S}}^{c} \cap \mathcal{E}_{\mathbf{X}_{1}}^{c} \cap \mathcal{E}_{\mathbf{X}_{2}}^{c}$. The 1.h.s. can be lower bounded by $(1-3 \epsilon) \leq$ $(1-\epsilon)^{3}$, and the r.h.s. can be upper bounded by $(1+\epsilon)^{3} \leq(1+4 \epsilon)$ whenever $\epsilon \leq 0.3$. Hence, for $\epsilon \leq 0.3$

$$
\left|\tilde{\rho}-\frac{\nu_{1} \nu_{2}}{\left\|\mathbf{u}_{1}^{*}\right\|\left\|\mathbf{u}_{2}^{*}\right\|}\left\langle\mathbf{s}_{1}, \mathbf{s}_{2}\right\rangle\right| \leq 4 \tilde{\rho} \epsilon \leq 4 \epsilon
$$

and thus

$$
\operatorname{Pr}\left[\mathcal{A}_{1} \mid \mathcal{E}_{\mathbf{S}}^{c} \cap \mathcal{E}_{\mathbf{X}_{1}}^{c} \cap \mathcal{E}_{\mathbf{X}_{2}}^{c}\right]=0
$$

Lemma 29 For every $\delta>0$ and $\epsilon>0$ there exists an $n_{\mathcal{A}}^{\prime}(\delta, \epsilon)$ such that for all $n>n_{\mathcal{A}}^{\prime}(\delta, \epsilon)$

$$
\operatorname{Pr}\left[\mathcal{A}_{2} \mid \mathcal{E}_{\mathbf{S}}^{c}\right]<\delta, \quad \operatorname{Pr}\left[\mathcal{A}_{3} \mid \mathcal{E}_{\mathbf{S}}^{c}\right]<\delta, \quad \operatorname{Pr}\left[\mathcal{A}_{4} \mid \mathcal{E}_{\mathbf{S}}^{c}\right]<\delta .
$$

Proof: We start with the derivation of the bound on $\mathcal{A}_{2}$. To this end, we first upper-bound the inner product between $\mathbf{s}_{1}$ and $\mathbf{w}_{2}$. Let $\mathbf{s}_{1, P}$ denote the projection of $\mathbf{s}_{1}$ onto the subspace of $\mathbb{R}^{n}$ that is orthogonal to $\mathbf{s}_{2}$, and that thus contains $\mathbf{w}_{2}$. Hence,

$$
\begin{aligned}
\left|\frac{\nu_{1}}{\left\|\mathbf{u}_{1}^{*}\right\|\left\|\mathbf{u}_{2}^{*}\right\|}\left\langle\mathbf{s}_{1}, \mathbf{w}_{2}\right\rangle\right| & \stackrel{(a)}{=}\left|\cos \varangle\left(\mathbf{s}_{1}, \mathbf{u}_{1}^{*}\right)\left\langle\frac{\mathbf{s}_{1}}{\left\|\mathbf{s}_{1}\right\|}, \frac{\mathbf{w}_{2}}{\left\|\mathbf{u}_{2}^{*}\right\|}\right\rangle\right| \\
& \stackrel{(b)}{\leq}\left|\cos \varangle\left(\mathbf{s}_{1}, \mathbf{u}_{1}^{*}\right)\right|\left|\left\langle\frac{\mathbf{s}_{1}}{\left\|\mathbf{s}_{1}\right\|}, \frac{\mathbf{w}_{2}}{\left\|\mathbf{w}_{2}\right\|}\right\rangle\right| \\
& \leq\left|\left\langle\frac{\mathbf{s}_{1}}{\left\|\mathbf{s}_{1}\right\|}, \frac{\mathbf{w}_{2}}{\left\|\mathbf{w}_{2}\right\|}\right\rangle\right|
\end{aligned}
$$




$$
\begin{aligned}
& =\left|\left\langle\frac{\mathbf{s}_{1, P}}{\left\|\mathbf{s}_{1}\right\|}, \frac{\mathbf{w}_{2}}{\left\|\mathbf{w}_{2}\right\|}\right\rangle\right| \\
& \leq\left|\left\langle\frac{\mathbf{s}_{1, P}}{\left\|\mathbf{s}_{1, P}\right\|}, \frac{\mathbf{w}_{2}}{\left\|\mathbf{w}_{2}\right\|}\right\rangle\right| \\
& =\left|\cos \varangle\left(\mathbf{s}_{1, P}, \mathbf{w}_{2}\right)\right|,
\end{aligned}
$$

where (a) follows by the definition of $\nu_{1}$ and (b) follows since by the definition of $\mathbf{w}_{2}$ we have $\left\|\mathbf{w}_{2}\right\| \leq\left\|\mathbf{u}_{2}^{*}\right\|$. By (207) it now follows that

$$
\begin{aligned}
& \operatorname{Pr}\left[\mathcal{A}_{2} \mid \mathcal{E}_{\mathbf{S}}^{c}\right] \leq \operatorname{Pr}\left[\left(\mathbf{S}_{1}, \mathbf{S}_{2}, \mathscr{C}_{1}, \mathscr{C}_{2}, \mathscr{C}_{c}\right):\left|\cos \varangle\left(\mathbf{S}_{1, P}, \mathbf{W}_{2}\right)\right|>\epsilon \mid \mathcal{E}_{\mathbf{S}}^{c}\right] \\
& \quad=\mathrm{E}_{\mathbf{S}_{1}, \mathbf{S}_{2}}\left[\operatorname{Pr}_{\mathscr{C}_{1}, \mathscr{C}_{2}, \mathscr{C}_{c}}\left(\left|\cos \varangle\left(\mathbf{S}_{1, P}, \mathbf{W}_{2}\right)\right|>\epsilon \mid\left(\mathbf{S}_{1}, \mathbf{S}_{2}\right)=\left(\mathbf{s}_{1}, \mathbf{S}_{2}\right), \mathcal{E}_{\mathbf{S}}^{c}\right)\right],
\end{aligned}
$$

where in the last line we have denoted by $\operatorname{Pr}_{\mathscr{C}_{1}, \mathscr{C}_{2}, \mathscr{C}_{c}}(\cdot \mid \cdot)$ the conditional probability of the codebooks $\mathscr{C}_{1}, \mathscr{C}_{2}$ and $\mathscr{C}_{3}$ being such that $\left|\cos \varangle\left(\mathbf{S}_{1, P}, \mathbf{W}_{2}\right)\right|>\epsilon$, given $\left(\mathbf{S}_{1}, \mathbf{S}_{2}\right)=\left(\mathbf{s}_{1}, \mathbf{s}_{2}\right)$ and $\mathcal{E}_{\mathbf{S}}^{c}$. To conclude our bound we now notice that conditioned on $\left(\mathbf{S}_{1}, \mathbf{S}_{2}\right)=\left(\mathbf{s}_{1}, \mathbf{s}_{2}\right)$, the random vector $\mathbf{W}_{2} /\left\|\mathbf{W}_{2}\right\|$ is distributed uniformly on the surface of the centered $\mathbb{R}^{n-1}$-sphere of unit radius, that lies in the subspace that is orthogonal to $\mathbf{s}_{2}$. Hence, by [11, Lemma B.1]

$$
\begin{aligned}
\operatorname{Pr}\left[\mathcal{A}_{2} \mid \mathcal{E}_{\mathbf{S}}^{c}\right] & \leq E_{\mathbf{S}_{1}, \mathbf{S}_{2}}\left[\frac{2 C_{n-1}(\Theta)}{C_{n-1}(\pi)} \mid \mathcal{E}_{\mathbf{S}}^{c}\right] \\
& \leq \frac{2 C_{n-1}(\Theta)}{C_{n-1}(\pi)} \\
& \stackrel{(a)}{\leq} \frac{2 \Gamma\left(\frac{n+1}{2}\right)}{(n-1) \Gamma\left(\frac{n}{2}\right) \sqrt{\pi}} \frac{\sin ^{(n-2)}(\Theta)}{\cos (\Theta)} \\
& \leq \frac{2 \Gamma\left(\frac{n+1}{2}\right)}{(n-1) \Gamma\left(\frac{n}{2}\right) \sqrt{\pi} \cos (\Theta)},
\end{aligned}
$$

where $\Theta \triangleq \arccos (\epsilon)$, and where in (a) we have used Lemma 25

Upper bounding the ratio of Gamma functions by the asymptotic series of Lemma 26, gives for every $\epsilon>0$ that $\operatorname{Pr}\left[\mathcal{A}_{2} \mid \mathcal{E}_{\mathbf{S}}^{c}\right] \rightarrow 0$ as $n \rightarrow \infty$. By similar arguments it also follows that $\operatorname{Pr}\left[\mathcal{A}_{3} \mid \mathcal{E}_{\mathbf{S}}^{c}\right] \rightarrow 0$ as $n \rightarrow \infty$.

To conclude the proof of Lemma 29, we derive the bound on $\mathcal{A}_{4}$. The derivations are similar to those for $\mathcal{A}_{2}$. First, let $\mathbf{w}_{1, P}$ denote the projection of $\mathbf{w}_{1}$ onto the subspace of $\mathbb{R}^{n}$ that is orthogonal to $\mathbf{s}_{2}$, and that thus contains $\mathbf{w}_{2}$. As in (207), we can show that

$$
\left|\frac{\nu_{1}}{\left\|\mathbf{u}_{1}^{*}\right\|\left\|\mathbf{u}_{2}^{*}\right\|}\left\langle\mathbf{w}_{1}, \mathbf{w}_{2}\right\rangle\right| \leq\left|\cos \varangle\left(\mathbf{w}_{1, P}, \mathbf{w}_{2}\right)\right| .
$$

Consequently,

$$
\begin{aligned}
& \operatorname{Pr}\left[\mathcal{A}_{4} \mid \mathcal{E}_{\mathbf{S}}^{c}\right] \leq \operatorname{Pr}\left[\left(\mathbf{S}_{1}, \mathbf{S}_{2}, \mathscr{C}_{1}, \mathscr{C}_{2}, \mathscr{C}_{c}\right):\left|\cos \varangle\left(\mathbf{W}_{1, P}, \mathbf{W}_{2}\right)\right|>\epsilon \mid \mathcal{E}_{\mathbf{S}}^{c}\right] \\
& \quad=\mathrm{E}_{\mathbf{S}_{1}, \mathbf{S}_{2}, \mathscr{C}_{1}}\left[\operatorname{Pr}_{\mathscr{C}_{2}, \mathscr{C}_{c}}\left(\left|\cos \varangle\left(\mathbf{W}_{1, P}, \mathbf{W}_{2}\right)\right|>\epsilon \mid\left(\mathbf{S}_{1}, \mathbf{S}_{2}, \mathbf{U}_{1}\right)=\left(\mathbf{s}_{1}, \mathbf{s}_{2}, \mathbf{u}_{1}\right), \mathcal{E}_{\mathbf{S}}^{c}\right)\right],
\end{aligned}
$$

where in the last line we have denoted by $\operatorname{Pr}_{\mathscr{C}_{2}, \mathscr{C}_{c}}(\cdot \mid \cdot)$ the conditional probability of the codebooks $\mathscr{C}_{2}$ and $\mathscr{C}_{3}$ being such that $\left|\cos \varangle\left(\mathbf{W}_{1, P}, \mathbf{W}_{2}\right)\right|>\epsilon$, given $\left(\mathbf{S}_{1}, \mathbf{S}_{2}, \mathbf{U}_{1}\right)=\left(\mathbf{s}_{1}, \mathbf{s}_{2}, \mathbf{u}_{1}\right)$ (hence also given $\mathbf{W}_{1, P}$ ) and $\mathcal{E}_{\mathbf{S}}^{c}$.

The desired upper bound now follows by noticing that conditioned on $\left(\mathbf{S}_{1}, \mathbf{S}_{2}, \mathbf{U}_{1}\right)=\left(\mathbf{s}_{1}, \mathbf{s}_{2}, \mathbf{u}_{1}\right)$, and $\mathscr{C}_{1}=\mathcal{C}_{1}$, the random vector $\mathbf{W}_{2} /\left\|\mathbf{W}_{2}\right\|$ is distributed uniformly on the surface of the centered $\mathbb{R}^{n-1}$-sphere of unit radius, that lies in the subspace that is orthogonal to $\mathbf{s}_{2}$. Hence, similarly as in 208

$$
\operatorname{Pr}\left[\mathcal{A}_{4} \mid \mathcal{E}_{\mathbf{S}}^{c}\right] \leq \mathrm{E}_{\mathbf{S}_{1}, \mathbf{S}_{2}, \mathscr{C}_{1}}\left[\frac{2 C_{n-1}(\Theta)}{C_{n-1}(\pi)} \mid \mathcal{E}_{\mathbf{S}}^{c}\right] .
$$


Therefore, for every $\epsilon>0, \operatorname{Pr}\left[\mathcal{A}_{4} \mid \mathcal{E}_{\mathbf{S}}^{c}\right] \rightarrow 0$ as $n \rightarrow \infty$.

Combining Lemma 28 and Lemma 29 with 205) gives that for every $\delta>0$ and $0<\epsilon<0.3$ there exists an $n_{\mathcal{A}}^{\prime}(\delta, \epsilon)$ such that for all $n>n_{\mathcal{A}}^{\prime}(\delta, \epsilon)$

$$
\operatorname{Pr}\left[\mathcal{E}_{\left(\mathbf{X}_{1}, \mathbf{X}_{2}\right)} \cap \mathcal{E}_{\mathbf{X}_{1}}^{c} \cap \mathcal{E}_{\mathbf{X}_{2}}^{c} \cap \mathcal{E}_{\mathbf{S}}^{c}\right] \leq 3 \delta .
$$

Proof of inequality (198): By the notation in (236) we have

$$
\begin{aligned}
\cos \varangle\left(\mathbf{u}_{1}^{*}, \mathbf{v}^{*}\right) & =\frac{\left\langle\mathbf{u}_{1}^{*}, \mathbf{v}^{*}\right\rangle}{\left\|\mathbf{u}_{1}^{*}\right\|\left\|\mathbf{v}^{*}\right\|} \\
& =\frac{\left\langle\mathbf{u}_{1}^{*}, \nu_{3} \mathbf{z}_{\mathrm{Q}_{1}}+\mathbf{w}_{3}\right\rangle}{\left\|\mathbf{u}_{1}^{*}\right\|\left\|\mathbf{v}^{*}\right\|} \\
& =\frac{\nu_{3}\left\langle\mathbf{u}_{1}^{*}, \mathbf{z}_{\mathrm{Q}_{1}}\right\rangle+\left\langle\mathbf{u}_{1}^{*}, \mathbf{w}_{3}\right\rangle}{\left\|\mathbf{u}_{1}^{*}\right\|\left\|\mathbf{v}^{*}\right\|},
\end{aligned}
$$

where we recall that $\nu_{3}$ is a function of $\left\|\mathbf{z}_{\mathrm{Q}_{1}}\right\|$ and $\cos \varangle\left(\mathbf{z}_{\mathrm{Q}_{1}}, \mathbf{v}^{*}\right)$. Now, define the two events

$$
\begin{aligned}
& \mathcal{A}_{1}=\left\{\left(\mathbf{s}_{1}, \mathbf{s}_{2}, \mathcal{C}_{1}, \mathcal{C}_{2}, \mathcal{C}_{c}\right):\left|\frac{\nu_{3}}{\left\|\mathbf{u}_{1}^{*}\right\|\left\|\mathbf{v}^{*}\right\|}\left\langle\mathbf{u}_{1}^{*}, \mathbf{z}_{\mathrm{Q}_{1}}\right\rangle\right|>2 \epsilon\right\} \\
& \mathcal{A}_{2}=\left\{\left(\mathbf{s}_{1}, \mathbf{s}_{2}, \mathcal{C}_{1}, \mathcal{C}_{2}, \mathcal{C}_{c}\right):\left|\frac{\nu_{1}}{\left\|\mathbf{u}_{1}^{*}\right\|\left\|\mathbf{v}^{*}\right\|}\left\langle\mathbf{u}_{1}^{*}, \mathbf{w}_{3}\right\rangle\right|>\epsilon\right\} .
\end{aligned}
$$

Note that by (210), $\mathcal{E}_{\left(\mathbf{x}_{1}, \mathbf{x}_{v}\right)}=\left\{\left(\mathbf{s}_{1}, \mathbf{s}_{2}, \mathcal{C}_{1}, \mathcal{C}_{2}, \mathcal{C}_{c}\right):\left|\cos \varangle\left(\mathbf{u}_{1}^{*}, \mathbf{v}^{*}\right)\right|>3 \epsilon\right\} \subset\left(\mathcal{A}_{1} \cup \mathcal{A}_{2}\right)$. Thus,

$$
\begin{aligned}
\operatorname{Pr} & {\left[\mathcal{E}_{\left(\mathbf{X}_{1}, \mathbf{X}_{v}\right)} \cap \mathcal{E}_{\mathbf{X}_{1}}^{c} \cap \mathcal{E}_{\mathbf{X}_{v}}^{c} \cap \mathcal{E}_{\mathbf{S}}^{c}\right] } \\
& \leq \operatorname{Pr}\left[\mathcal{A}_{1} \cap \mathcal{E}_{\mathbf{X}_{1}}^{c} \cap \mathcal{E}_{\mathbf{X}_{v}}^{c} \cap \mathcal{E}_{\mathbf{S}}^{c}\right]+\operatorname{Pr}\left[\mathcal{A}_{2} \cap \mathcal{E}_{\mathbf{X}_{1}}^{c} \cap \mathcal{E}_{\mathbf{X}_{v}}^{c} \cap \mathcal{E}_{\mathbf{S}}^{c}\right] \\
& \leq \operatorname{Pr}\left[\mathcal{A}_{1} \mid \mathcal{E}_{\mathbf{X}_{1}}^{c} \cap \mathcal{E}_{\mathbf{X}_{v}}^{c} \cap \mathcal{E}_{\mathbf{S}}^{c}\right]+\operatorname{Pr}\left[\mathcal{A}_{2} \mid \mathcal{E}_{\mathbf{S}}^{c}\right] .
\end{aligned}
$$

The two terms on the r.h.s. of (211) are now bounded in the following two lemmas.

Lemma 30 For $0<\epsilon \leq 1$

$$
\operatorname{Pr}\left[\mathcal{A}_{1} \mid \mathcal{E}_{\mathbf{S}}^{c} \cap \mathcal{E}_{\mathbf{X}_{1}}^{c} \cap \mathcal{E}_{\mathbf{X}_{v}}^{c}\right]=0
$$

Proof: We first note that the term in the definition of $\mathcal{A}_{1}$ can be rewritten as

$$
\frac{\nu_{3}}{\left\|\mathbf{u}_{1}^{*}\right\|\left\|\mathbf{v}^{*}\right\|}\left\langle\mathbf{u}_{1}^{*}, \mathbf{z}_{\mathrm{Q}_{1}}\right\rangle=\cos \varangle\left(\mathbf{z}_{\mathrm{Q}_{1}}, \mathbf{v}^{*}\right) \cos \varangle\left(\mathbf{u}_{1}^{*}, \mathbf{z}_{\mathrm{Q}_{1}}\right) .
$$

We can now upper and lower bound the r.h.s. of (212) for $\left(\mathbf{s}_{1}, \mathbf{s}_{2}, \mathcal{C}_{1}, \mathcal{C}_{2}, \mathcal{C}_{c}\right) \in \mathcal{E}_{\mathbf{S}}^{c} \cap \mathcal{E}_{\mathbf{X}_{2}}^{c} \cap \mathcal{E}_{\mathbf{X}_{v}}^{c}$ by noticing that $\left(\mathbf{s}_{1}, \mathbf{s}_{2}, \mathcal{C}_{1}, \mathcal{C}_{2}, \mathcal{C}_{c}\right) \in \mathcal{E}_{\mathbf{X}_{1}}^{c}$ implies

$$
\left|\cos \varangle\left(\mathbf{u}_{1}^{*}, \mathbf{z}_{\mathbf{Q}_{1}}\right)\right|<\epsilon,
$$

and that $\left(\mathbf{s}_{1}, \mathbf{s}_{2}, \mathcal{C}_{1}, \mathcal{C}_{2}, \mathcal{C}_{c}\right) \in \mathcal{E}_{\mathbf{X}_{v}}^{c}$ implies

$$
\left|\sqrt{1-2^{-2 R_{\mathrm{c}}}}-\cos \varangle\left(\mathbf{z}_{\mathrm{Q}_{1}}, \mathbf{v}^{*}\right)\right|<\epsilon \sqrt{1-2^{-2 R_{\mathrm{c}}}} .
$$

Hence, combined with (212) this gives

$$
\left|\frac{\nu_{3}}{\left\|\mathbf{u}_{1}^{*}\right\|\left\|\mathbf{v}^{*}\right\|}\left\langle\mathbf{u}_{1}^{*}, \mathbf{z}_{\mathrm{Q}_{1}}\right\rangle\right| \leq \sqrt{1-2^{-2 R_{\mathrm{c}}}} \epsilon(1+\epsilon),
$$


whenever $\left(\mathbf{s}_{1}, \mathbf{s}_{2}, \mathcal{C}_{1}, \mathcal{C}_{2}, \mathcal{C}_{c}\right) \in \mathcal{E}_{\mathbf{S}}^{c} \cap \mathcal{E}_{\mathbf{X}_{1}}^{c} \cap \mathcal{E}_{\mathbf{X}_{v}}^{c}$. The r.h.s. can be upper bounded by $\epsilon(1+\epsilon) \leq 2 \epsilon$ whenever $\epsilon \leq 1$. Hence, for $\epsilon \leq 1$

$$
\left|\frac{\nu_{3}}{\left\|\mathbf{u}_{1}^{*}\right\|\left\|\mathbf{v}^{*}\right\|}\left\langle\mathbf{u}_{1}^{*}, \mathbf{z}_{\mathrm{Q}_{1}}\right\rangle\right| \leq 2 \sqrt{1-2^{-2 R_{\mathrm{c}}}} \epsilon \leq 2 \epsilon,
$$

and thus

$$
\operatorname{Pr}\left[\mathcal{A}_{1} \mid \mathcal{E}_{\mathbf{S}}^{c} \cap \mathcal{E}_{\mathbf{X}_{1}}^{c} \cap \mathcal{E}_{\mathbf{X}_{v}}^{c}\right]=0 .
$$

Lemma 31 For every $\delta>0$ and $\epsilon>0$ there exists an $n_{\mathcal{A}}^{\prime}(\delta, \epsilon)$ such that for all $n>n_{\mathcal{A}}^{\prime}(\delta, \epsilon)$

$$
\operatorname{Pr}\left[\mathcal{A}_{2} \mid \mathcal{E}_{\mathbf{S}}^{c}\right]<\delta .
$$

Proof: We first upper-bound the inner product between $\mathbf{u}_{1}^{*}$ and $\mathbf{w}_{3}$. Let $\mathbf{u}_{1, \mathrm{P}}$ denote the projection of $\mathbf{u}_{1}^{*}$ onto the subspace of $\mathbb{R}^{n}$ that is orthogonal to $\mathbf{z}_{\mathrm{Q}_{1}}$, and therfore contains $\mathbf{w}_{3}$. Hence,

$$
\begin{aligned}
\left|\frac{\nu_{1}}{\left\|\mathbf{u}_{1}^{*}\right\|\left\|\mathbf{v}^{*}\right\|}\left\langle\mathbf{u}_{1}^{*}, \mathbf{w}_{3}\right\rangle\right| & \stackrel{(a)}{=}\left|\cos \varangle\left(\mathbf{s}_{1}, \mathbf{u}_{1}^{*}\right)\left\langle\frac{\mathbf{u}_{1}^{*}}{\left\|\mathbf{s}_{1}\right\|}, \frac{\mathbf{w}_{3}}{\left\|\mathbf{v}^{*}\right\|}\right\rangle\right| \\
& \stackrel{(b)}{\leq}\left|\cos \varangle\left(\mathbf{s}_{1}, \mathbf{u}_{1}^{*}\right)\right|\left\langle\frac{\mathbf{u}_{1}^{*}}{\left\|\mathbf{s}_{1}\right\|}, \frac{\mathbf{w}_{3}}{\left\|\mathbf{w}_{3}\right\|}\right\rangle \mid \\
& \leq\left|\left\langle\frac{\mathbf{u}_{1}^{*}}{\left\|\mathbf{s}_{1}\right\|}, \frac{\mathbf{w}_{3}}{\left\|\mathbf{w}_{3}\right\|}\right\rangle\right| \\
& =\left|\left\langle\frac{\mathbf{u}_{1, \mathrm{P}}}{\left\|\mathbf{u}_{1}^{*}\right\|}, \frac{\mathbf{w}_{3}}{\left\|\mathbf{w}_{3}\right\|}\right\rangle\right| \\
& \leq\left|\left\langle\frac{\mathbf{u}_{1, \mathrm{P}}}{\left\|\mathbf{u}_{1, \mathrm{P}}\right\|}, \frac{\mathbf{w}_{3}}{\left\|\mathbf{w}_{3}\right\|}\right\rangle\right| \\
& =\left|\cos \varangle\left(\mathbf{u}_{1, \mathrm{P}}, \mathbf{w}_{3}\right)\right|,
\end{aligned}
$$

where (a) follows by the definition of $\nu_{1}$ and (b) follows since by the definition of $\mathbf{w}_{3}$ we have $\left\|\mathbf{w}_{3}\right\| \leq\left\|\mathbf{v}^{*}\right\|$. By (213) it now follows that

$$
\begin{aligned}
\operatorname{Pr}\left[\mathcal{A}_{2} \mid \mathcal{E}_{\mathbf{S}}^{c}\right] & \leq \operatorname{Pr}\left[\left(\mathbf{S}_{1}, \mathbf{S}_{2}, \mathscr{C}_{1}, \mathscr{C}_{2}, \mathscr{C}_{c}\right):\left|\cos \varangle\left(\mathbf{U}_{1, \mathrm{P}}, \mathbf{W}_{3}\right)\right|>\epsilon \mid \mathcal{E}_{\mathbf{S}}^{c}\right] \\
& \stackrel{(a)}{=} \mathrm{E}_{\mathbf{S}_{1}, \mathbf{S}_{2}, \mathscr{C}_{1}}\left[\operatorname{Pr}_{\mathscr{C}_{2}, \mathscr{C}_{c}}\left(\left|\cos \varangle\left(\mathbf{u}_{1, P}, \mathbf{W}_{3}\right)\right|>\epsilon \mid\left(\mathbf{S}_{1}, \mathbf{S}_{2}, \mathbf{U}_{1}\right)=\left(\mathbf{s}_{1}, \mathbf{s}_{2}, \mathbf{u}_{1}\right), \mathcal{E}_{\mathbf{S}}^{c}\right)\right],
\end{aligned}
$$

where $0<\epsilon \leq 1$, and where in (a) we have denoted by $\operatorname{Pr}_{c_{2}, c_{c}}(\cdot \mid \cdot)$ the conditional probability of the codebooks $c_{2}$ and $c_{c}$ being such that $\left|\cos \varangle\left(\mathbf{u}_{1, P}, \mathbf{W}_{3}\right)\right|>\epsilon$, given $\left(\mathbf{S}_{1}, \mathbf{S}_{2}, \mathbf{U}_{1}\right)=$ $\left(\mathbf{s}_{1}, \mathbf{s}_{2}, \mathbf{u}_{1}\right)$ and $\mathcal{E}_{\mathbf{S}}^{c}$. To conclude our bound, we now notice that conditioned on $\left(\mathbf{S}_{1}, \mathbf{S}_{2}, \mathbf{U}_{1}\right)=$ $\left(\mathbf{s}_{1}, \mathbf{s}_{2}, \mathbf{u}_{1}\right)$, the random vector $\mathbf{W}_{3} /\left\|\mathbf{W}_{3}\right\|$ is distributed uniformly on the surface of the centered $\mathbb{R}^{n-1}$-sphere of unit radius, that lies in the subspace that is orthogonal to $\mathbf{z}_{\mathrm{Q}_{1}}$. Hence, according to [11, Lemma B.1],

$$
\begin{aligned}
\operatorname{Pr}\left[\mathcal{A}_{2} \mid \mathcal{E}_{\mathbf{S}}^{c}\right] & \leq \mathrm{E}_{\mathbf{S}_{1}, \mathbf{S}_{2}, \mathscr{C}_{1}}\left[\frac{2 C_{n-1}(\Theta)}{C_{n-1}(\pi)} \mid \mathcal{E}_{\mathbf{S}}^{c}\right] \\
& \leq \frac{2 C_{n-1}(\Theta)}{C_{n-1}(\pi)}
\end{aligned}
$$

where $\Theta \triangleq \arccos (\epsilon)$. Note that as $0<\epsilon \leq 1, \Theta \in\left(0, \frac{\pi}{2}\right)$, and thus, by [11, Lemma B.4], the r.h.s. of (214) tends to 0 as $n \rightarrow \infty$, and therefore $\operatorname{Pr}\left[\mathcal{A}_{2} \mid \mathcal{E}_{\mathbf{S}}^{c}\right] \rightarrow 0$. 
Combining Lemma 30 and Lemma 31 with (211) gives that for every $\delta>0$ and $0<\epsilon \leq 1$ there exists an $n_{\mathcal{A}}^{\prime}(\delta, \epsilon)$ such that for all $n>n_{\mathcal{A}}^{\prime}(\delta, \epsilon)$

$$
\operatorname{Pr}\left[\mathcal{E}_{\left(\mathbf{X}_{1}, \mathbf{X}_{v}\right)} \cap \mathcal{E}_{\mathbf{X}_{2}}^{c} \cap \mathcal{E}_{\mathbf{X}_{v}}^{c} \cap \mathcal{E}_{\mathbf{S}}^{c}\right] \leq \delta .
$$

Proof of inequality (199): By the notation in 236) we have

$$
\begin{aligned}
\cos \varangle\left(\mathbf{u}_{2}^{*}, \mathbf{v}^{*}\right) & =\frac{\left\langle\mathbf{u}_{2}^{*}, \mathbf{v}^{*}\right\rangle}{\left\|\mathbf{u}_{2}^{*}\right\|\left\|\mathbf{v}^{*}\right\|} \\
& =\frac{\left\langle\nu_{2} \mathbf{s}_{2}+\mathbf{w}_{2}, \nu_{3} \mathbf{z}_{\mathrm{Q}_{1}}+\mathbf{w}_{3}\right\rangle}{\left\|\mathbf{u}_{2}^{*}\right\|\left\|\mathbf{v}^{*}\right\|} \\
& =\frac{\nu_{2} \nu_{3}\left\langle\mathbf{s}_{2}, \mathbf{z}_{\mathrm{Q}_{1}}\right\rangle+\nu_{2}\left\langle\mathbf{s}_{2}, \mathbf{w}_{3}\right\rangle+\nu_{3}\left\langle\mathbf{z}_{\mathrm{Q}_{1}}, \mathbf{w}_{2}\right\rangle+\left\langle\mathbf{w}_{2}, \mathbf{w}_{3}\right\rangle}{\left\|\mathbf{u}_{2}^{*}\right\|\left\|\mathbf{v}^{*}\right\|},
\end{aligned}
$$

where we recall that $\nu_{2}$ is a function of $\left\|\mathbf{s}_{2}\right\|$ and $\cos \varangle\left(\mathbf{s}_{2}, \mathbf{u}_{2}^{*}\right)$ and similarly $\nu_{3}$ is a function of $\left\|\mathbf{z}_{\mathrm{Q}_{1}}\right\|$ and $\cos \varangle\left(\mathbf{z}_{\mathrm{Q}_{1}}, \mathbf{v}^{*}\right)$. Now, define the four events

$$
\begin{aligned}
& \mathcal{A}_{1}=\left\{\left(\mathbf{s}_{1}, \mathbf{s}_{2}, \mathcal{C}_{1}, \mathcal{C}_{2}, \mathcal{C}_{c}\right):\left|\bar{\rho}-\frac{\nu_{2} \nu_{3}}{\left\|\mathbf{u}_{2}^{*}\right\|\left\|\mathbf{v}^{*}\right\|}\left\langle\mathbf{s}_{2}, \mathbf{z}_{\mathrm{Q}_{1}}\right\rangle\right|>4 \epsilon\right\} \\
& \mathcal{A}_{2}=\left\{\left(\mathbf{s}_{1}, \mathbf{s}_{2}, \mathcal{C}_{1}, \mathcal{C}_{2}, \mathcal{C}_{c}\right):\left|\frac{\nu_{2}}{\left\|\mathbf{u}_{2}^{*}\right\|\left\|\mathbf{v}^{*}\right\|}\left\langle\mathbf{s}_{2}, \mathbf{w}_{3}\right\rangle\right|>\epsilon\right\} \\
& \mathcal{A}_{3}=\left\{\left(\mathbf{s}_{1}, \mathbf{s}_{2}, \mathcal{C}_{1}, \mathcal{C}_{2}, \mathcal{C}_{c}\right):\left|\frac{\nu_{2}}{\left\|\mathbf{u}_{2}^{*}\right\|\left\|\mathbf{v}^{*}\right\|}\left\langle\mathbf{z}_{\mathrm{Q}_{1}}, \mathbf{w}_{2}\right\rangle\right|>\epsilon\right\} \\
& \mathcal{A}_{4}=\left\{\left(\mathbf{s}_{1}, \mathbf{s}_{2}, \mathcal{C}_{1}, \mathcal{C}_{2}, \mathcal{C}_{c}\right):\left|\frac{1}{\left\|\mathbf{u}_{2}^{*}\right\|\left\|\mathbf{v}^{*}\right\|}\left\langle\mathbf{w}_{2}, \mathbf{w}_{3}\right\rangle\right|>\epsilon\right\} .
\end{aligned}
$$

Note that by (215),

$$
\begin{aligned}
\mathcal{E}_{\left(\mathbf{X}_{2}, \mathbf{X}_{v}\right)}=\left\{\left(\mathbf{s}_{1}, \mathbf{s}_{2}, \mathcal{C}_{1}, \mathcal{C}_{2}, \mathcal{C}_{c}\right):\left|\bar{\rho}-\cos \varangle\left(\mathbf{u}_{2}^{*}, \mathbf{v}^{*}\right)\right|>7 \epsilon\right\} \subset\left(\mathcal{A}_{1} \cup \mathcal{A}_{2} \cup \mathcal{A}_{3} \cup \mathcal{A}_{4}\right) . \text { Thus, } \\
\operatorname{Pr}\left[\mathcal{E}_{\left(\mathbf{X}_{2}, \mathbf{X}_{v}\right)} \cap \mathcal{E}_{\mathbf{X}_{2}}^{c} \cap \mathcal{E}_{\mathbf{X}_{v}}^{c} \cap \mathcal{E}_{\mathbf{S}}^{c}\right] \\
\leq \operatorname{Pr}\left[\mathcal{A}_{1} \cap \mathcal{E}_{\mathbf{X}_{2}}^{c} \cap \mathcal{E}_{\mathbf{X}_{v}}^{c} \cap \mathcal{E}_{\mathbf{S}}^{c}\right]+\operatorname{Pr}\left[\mathcal{A}_{2} \cap \mathcal{E}_{\mathbf{X}_{2}}^{c} \cap \mathcal{E}_{\mathbf{X}_{v}}^{c} \cap \mathcal{E}_{\mathbf{S}}^{c}\right] \\
\quad+\operatorname{Pr}\left[\mathcal{A}_{3} \cap \mathcal{E}_{\mathbf{X}_{2}}^{c} \cap \mathcal{E}_{\mathbf{X}_{v}}^{c} \cap \mathcal{E}_{\mathbf{S}}^{c}\right]+\operatorname{Pr}\left[\mathcal{A}_{4} \cap \mathcal{E}_{\mathbf{X}_{2}}^{c} \cap \mathcal{E}_{\mathbf{X}_{v}}^{c} \cap \mathcal{E}_{\mathbf{S}}^{c}\right] \\
\leq \operatorname{Pr}\left[\mathcal{A}_{1} \mid \mathcal{E}_{\mathbf{X}_{2}}^{c} \cap \mathcal{E}_{\mathbf{X}_{v}}^{c} \cap \mathcal{E}_{\mathbf{S}}^{c}\right]+\operatorname{Pr}\left[\mathcal{A}_{2} \mid \mathcal{E}_{\mathbf{S}}^{c}\right]+\operatorname{Pr}\left[\mathcal{A}_{3} \mid \mathcal{E}_{\mathbf{S}}^{c}\right]+\operatorname{Pr}\left[\mathcal{A}_{4} \mid \mathcal{E}_{\mathbf{S}}^{c}\right] .
\end{aligned}
$$

The four terms on the r.h.s. of (216) are now bounded in the following two lemmas.

Lemma 32 For $\epsilon<0.3$

$$
\operatorname{Pr}\left[\mathcal{A}_{1} \mid \mathcal{E}_{\mathbf{S}}^{c} \cap \mathcal{E}_{\mathbf{X}_{2}}^{c} \cap \mathcal{E}_{\mathbf{X}_{v}}^{c}\right]=0 .
$$

Proof: We first note that the term in the definition of $\mathcal{A}_{1}$ can be rewritten as

$$
\frac{\nu_{2} \nu_{3}}{\left\|\mathbf{u}_{2}^{*}\right\|\left\|\mathbf{v}^{*}\right\|}\left\langle\mathbf{s}_{2}, \mathbf{z}_{\mathrm{Q}_{1}}\right\rangle=\cos \varangle\left(\mathbf{s}_{2}, \mathbf{u}_{2}^{*}\right) \cos \varangle\left(\mathbf{z}_{\mathrm{Q}_{1}}, \mathbf{v}^{*}\right) \cos \varangle\left(\mathbf{s}_{2}, \mathbf{z}_{\mathrm{Q}_{1}}\right) \text {. }
$$

We can now upper and lower bound the r.h.s. of 217) for $\left(\mathbf{s}_{1}, \mathbf{s}_{2}, \mathcal{C}_{1}, \mathcal{C}_{2}, \mathcal{C}_{c}\right) \in \mathcal{E}_{\mathbf{S}}^{c} \cap \mathcal{E}_{\mathbf{X}_{2}}^{c} \cap \mathcal{E}_{\mathbf{X}_{v}}^{c}$ by noticing that $\left(\mathbf{s}_{1}, \mathbf{s}_{2}, \mathcal{C}_{1}, \mathcal{C}_{2}, \mathcal{C}_{c}\right) \in \mathcal{E}_{\mathbf{S}}^{c}$ implies

$$
\left|\rho \sqrt{2^{-2 R_{1}}}-\cos \varangle\left(\mathbf{s}_{2}, \mathbf{z}_{\mathrm{Q}_{1}}\right)\right|<\rho \sqrt{2^{-2 R_{1}}} \epsilon,
$$

that $\left(\mathbf{s}_{1}, \mathbf{s}_{2}, \mathcal{C}_{1}, \mathcal{C}_{2}, \mathcal{C}_{c}\right) \in \mathcal{E}_{\mathbf{X}_{2}}^{c}$ implies

$$
\left|\sqrt{1-2^{-2 R_{2}}}-\cos \varangle\left(\mathbf{s}_{2}, \mathbf{u}_{2}^{*}\right)\right|<\epsilon \sqrt{1-2^{-2 R_{2}}},
$$


and that $\left(\mathbf{s}_{1}, \mathbf{s}_{2}, \mathcal{C}_{1}, \mathcal{C}_{2}, \mathcal{C}_{c}\right) \in \mathcal{E}_{\mathbf{X}_{v}}^{c}$ implies

$$
\left|\sqrt{1-2^{-2 R_{\mathrm{c}}}}-\cos \varangle\left(\mathbf{z}_{\mathrm{Q}_{1}}, \mathbf{v}^{*}\right)\right|<\epsilon \sqrt{1-2^{-2 R_{\mathrm{c}}}} .
$$

Hence, combined with (217) this gives

$$
\bar{\rho}(1-\epsilon)^{3} \leq \frac{\nu_{1} \nu_{2}}{\left\|\mathbf{u}_{1}^{*}\right\|\left\|\mathbf{u}_{2}^{*}\right\|}\left\langle\mathbf{s}_{1}, \mathbf{s}_{2}\right\rangle \leq \bar{\rho}(1+\epsilon)^{3},
$$

whenever $\left(\mathbf{s}_{1}, \mathbf{s}_{2}, \mathcal{C}_{1}, \mathcal{C}_{2}, \mathcal{C}_{c}\right) \in \mathcal{E}_{\mathbf{S}}^{c} \cap \mathcal{E}_{\mathbf{X}_{2}}^{c} \cap \mathcal{E}_{\mathbf{X}_{v}}^{c}$. The 1.h.s. can be lower bounded by $(1-3 \epsilon) \leq$ $(1-\epsilon)^{3}$, and the r.h.s. can be upper bounded by $(1+\epsilon)^{3} \leq(1+4 \epsilon)$ whenever $\epsilon \leq 0.3$. Hence, for $\epsilon \leq 0.3$

$$
\left|\bar{\rho}-\frac{\nu_{1} \nu_{2}}{\left\|\mathbf{u}_{1}^{*}\right\|\left\|\mathbf{u}_{2}^{*}\right\|}\left\langle\mathbf{s}_{1}, \mathbf{s}_{2}\right\rangle\right| \leq 4 \bar{\rho} \epsilon \leq 4 \epsilon,
$$

and thus

$$
\operatorname{Pr}\left[\mathcal{A}_{1} \mid \mathcal{E}_{\mathbf{S}}^{c} \cap \mathcal{E}_{\mathbf{X}_{2}}^{c} \cap \mathcal{E}_{\mathbf{X}_{v}}^{c}\right]=0 .
$$

Remark 6 To show (218), note that

$$
\begin{aligned}
\cos \varangle\left(\mathbf{s}_{2}, \mathbf{z}_{Q_{1}}\right) & =\frac{\left\langle\mathbf{s}_{2}, \mathbf{z}_{\mathrm{Q}_{1}}\right\rangle}{\left\|\mathbf{s}_{2}\right\|\left\|\mathbf{z}_{\mathrm{Q}_{1}}\right\|}=\frac{\left\langle\mathbf{s}_{2}, \mathbf{s}_{1}-\mathbf{u}_{1}^{*}\right\rangle}{\left\|\mathbf{s}_{2}\right\|\left\|\mathbf{z}_{\mathrm{Q}_{1}}\right\|}=\frac{\left\langle\mathbf{s}_{2}, \mathbf{s}_{1}\right\rangle-\left\langle\mathbf{s}_{2}, \mathbf{u}_{1}^{*}\right\rangle}{\left\|\mathbf{s}_{2}\right\|\left\|\mathbf{z}_{\mathrm{Q}_{1}}\right\|} \\
& =\frac{\left\langle\mathbf{s}_{2}, \mathbf{s}_{1}\right\rangle-\left\langle\mathbf{s}_{2}, \nu_{1} \mathbf{s}_{1}+\mathbf{w}_{1}\right\rangle}{\left\|\mathbf{s}_{2}\right\|\left\|\mathbf{z}_{\mathrm{Q}_{1}}\right\|} \\
& =\frac{\left(1-\nu_{1}\right)\left\langle\mathbf{s}_{2}, \mathbf{s}_{1}\right\rangle-\left\langle\mathbf{s}_{2}, \mathbf{w}_{1}\right\rangle}{\left\|\mathbf{s}_{2}\right\|\left\|\mathbf{z}_{\mathrm{Q}_{1}}\right\|}=\frac{2^{-2 R_{1}}\left\|\mathbf{s}_{1}\right\|\left\|\mathbf{s}_{2}\right\| \cos \varangle\left(\mathbf{s}_{1}, \mathbf{s}_{2}\right)}{\left\|\mathbf{s}_{2}\right\| \sqrt{n \sigma^{2} 2^{-2 R_{1}}}}-\frac{\left\langle\mathbf{s}_{2}, \mathbf{w}_{1}\right\rangle}{\left\|\mathbf{s}_{2}\right\|\left\|\mathbf{z}_{\mathrm{Q}_{1}}\right\|} \\
& =\sqrt{2^{-2 R_{1}}} \cos \varangle\left(\mathbf{s}_{1}, \mathbf{s}_{2}\right)-\frac{\left\langle\mathbf{s}_{2}, \mathbf{w}_{1}\right\rangle}{\left\|\mathbf{s}_{2}\right\|\left\|\mathbf{z}_{\mathrm{Q}_{1}}\right\|} .
\end{aligned}
$$

The second term vanishes when $n \rightarrow \infty$ as in proof of Lemma 29

Lemma 33 For every $\delta>0$ and $\epsilon>0$ there exists an $n_{\mathcal{A}}^{\prime}(\delta, \epsilon)$ such that for all $n>n_{\mathcal{A}}^{\prime}(\delta, \epsilon)$

$$
\operatorname{Pr}\left[\mathcal{A}_{2} \mid \mathcal{E}_{\mathbf{S}}^{c}\right]<\delta, \quad \operatorname{Pr}\left[\mathcal{A}_{3} \mid \mathcal{E}_{\mathbf{S}}^{c}\right]<\delta, \quad \operatorname{Pr}\left[\mathcal{A}_{4} \mid \mathcal{E}_{\mathbf{S}}^{c}\right]<\delta .
$$

Proof: We start with the derivation of the bound on $\mathcal{A}_{2}$. To this end, we first upper-bound the inner product between $\mathbf{s}_{2}$ and $\mathbf{w}_{3}$. Let $\mathbf{s}_{2, P}$ denote the projection of $\mathbf{s}_{2}$ onto the subspace of $\mathbb{R}^{n}$ that is orthogonal to $\mathbf{z}_{\mathrm{Q}_{1}}$, and that thus contains $\mathbf{w}_{2}$. Hence,

$$
\begin{aligned}
\left|\frac{\nu_{2}}{\left\|\mathbf{u}_{2}^{*}\right\|\left\|\mathbf{v}^{*}\right\|}\left\langle\mathbf{s}_{2}, \mathbf{w}_{3}\right\rangle\right| & \stackrel{(a)}{=}\left|\cos \varangle\left(\mathbf{s}_{2}, \mathbf{u}_{2}^{*}\right)\left\langle\frac{\mathbf{s}_{2}}{\left\|\mathbf{s}_{2}\right\|}, \frac{\mathbf{w}_{3}}{\left\|\mathbf{v}^{*}\right\|}\right\rangle\right| \\
& \stackrel{(b)}{\leq}\left|\cos \varangle\left(\mathbf{s}_{2}, \mathbf{u}_{2}^{*}\right)\right|\left\langle\frac{\mathbf{s}_{2}}{\left\|\mathbf{s}_{2}\right\|}, \frac{\mathbf{w}_{3}}{\left\|\mathbf{w}_{3}\right\|}\right\rangle \mid \\
& \leq\left|\left\langle\frac{\mathbf{s}_{2}}{\left\|\mathbf{s}_{2}\right\|}, \frac{\mathbf{w}_{3}}{\left\|\mathbf{w}_{3}\right\|}\right\rangle\right| \\
& =\left|\left\langle\frac{\mathbf{s}_{2, \mathrm{P}}}{\left\|\mathbf{s}_{2}\right\|}, \frac{\mathbf{w}_{3}}{\left\|\mathbf{w}_{3}\right\|}\right\rangle\right| \\
& \leq\left|\left\langle\frac{\mathbf{s}_{2, \mathrm{P}}}{\left\|\mathbf{s}_{2, \mathrm{P}}\right\|}, \frac{\mathbf{w}_{3}}{\left\|\mathbf{w}_{3}\right\|}\right\rangle\right| \\
& =\left|\cos \varangle\left(\mathbf{s}_{2, \mathrm{P}}, \mathbf{w}_{3}\right)\right|,
\end{aligned}
$$


where (a) follows by the definition of $\nu_{2}$ and (b) follows since by the definition of $\mathbf{w}_{3}$ we have $\left\|\mathbf{w}_{3}\right\| \leq\left\|\mathbf{v}^{*}\right\|$. By (218) it now follows that

$$
\begin{aligned}
\operatorname{Pr}\left[\mathcal{A}_{2} \mid \mathcal{E}_{\mathbf{S}}^{c}\right] & \leq \operatorname{Pr}\left[\left(\mathbf{S}_{1}, \mathbf{S}_{2}, \mathscr{C}_{1}, \mathscr{C}_{2}, \mathscr{C}_{c}\right):\left|\cos \varangle\left(\mathbf{S}_{2, \mathrm{P}}, \mathbf{W}_{3}\right)\right|>\epsilon \mid \mathcal{E}_{\mathbf{S}}^{c}\right] \\
& \stackrel{(a)}{=} \mathrm{E}_{\mathbf{S}_{1}, \mathbf{S}_{2}}\left[\operatorname{Pr}_{\mathscr{C}_{1}, \mathscr{C}_{2}, \mathscr{C}_{c}}\left(\left|\cos \varangle\left(\mathbf{s}_{2, P}, \mathbf{W}_{3}\right)\right|>\epsilon \mid\left(\mathbf{S}_{1}, \mathbf{S}_{2}\right)=\left(\mathbf{s}_{1}, \mathbf{s}_{2}\right), \mathcal{E}_{\mathbf{S}}^{c}\right)\right],
\end{aligned}
$$

where in (a) we have denoted by $\operatorname{Pr}_{\mathscr{C}_{1}, \mathscr{C}_{2}, \mathscr{C}_{c}}(\cdot \mid \cdot)$ the conditional probability of the codebooks $c_{1}, c_{2}$ and $c_{3}$ being such that $\left|\cos \varangle\left(\mathbf{s}_{2, P}, \mathbf{W}_{3}\right)\right|>\epsilon$, given $\left(\mathbf{S}_{1}, \mathbf{S}_{2}\right)=\left(\mathbf{s}_{1}, \mathbf{s}_{2}\right)$ and $\mathcal{E}_{\mathbf{S}}^{c}$. To conclude our bound we now notice that conditioned on $\left(\mathbf{S}_{1}, \mathbf{S}_{2}\right)=\left(\mathbf{s}_{1}, \mathbf{s}_{2}\right)$, the random vector $\mathbf{W}_{3} /\left\|\mathbf{W}_{3}\right\|$ is distributed uniformly on the surface of the centered $\mathbb{R}^{n-1}$-sphere of unit radius, that lies in the subspace that is orthogonal to $\mathbf{s}_{2}$. Hence,

$$
\begin{aligned}
\operatorname{Pr}\left[\mathcal{A}_{2} \mid \mathcal{E}_{\mathbf{S}}^{c}\right] & \leq \mathrm{E}_{\mathbf{S}_{1}, \mathbf{S}_{2}}\left[\frac{2 C_{n-1}(\Theta)}{C_{n-1}(\pi)} \mid \mathcal{E}_{\mathbf{S}}^{c}\right] \\
& \leq \frac{2 C_{n-1}(\Theta)}{C_{n-1}(\pi)}
\end{aligned}
$$

where $\Theta \triangleq \arccos (\epsilon)$. As $0<\epsilon \leq 1, \Theta \in\left(0, \frac{\pi}{2}\right)$, and thus by [11, Lemma B.4] the r.h.s. of (219) tends to 0 as $n \rightarrow \infty$, and therefore $\operatorname{Pr}\left[\mathcal{A}_{2} \mid \mathcal{E}_{\mathbf{S}}^{c}\right] \rightarrow 0$. By similar arguments it also follows that $\operatorname{Pr}\left[\mathcal{A}_{3} \mid \mathcal{E}_{\mathbf{S}}^{c}\right] \rightarrow 0$ as $n \rightarrow \infty$.

To conclude the proof of Lemma 33, we derive the bound on $\mathcal{A}_{4}$. The derivations are similar to those for $\mathcal{A}_{2}$. First, let $\mathbf{w}_{2, P}$ denote the projection of $\mathbf{w}_{2}$ onto the subspace of $\mathbb{R}^{n}$ that is orthogonal to $\mathbf{z}_{\mathrm{Q}_{1}}$, and that thus contains $\mathbf{w}_{3}$. As in (218), we can show that

$$
\left|\frac{1}{\left\|\mathbf{u}_{2}^{*}\right\|\left\|\mathbf{v}^{*}\right\|}\left\langle\mathbf{w}_{2}, \mathbf{w}_{3}\right\rangle\right| \leq\left|\cos \varangle\left(\mathbf{w}_{2, P}, \mathbf{w}_{3}\right)\right|,
$$

from which it then follows that

$$
\operatorname{Pr}\left[\mathcal{A}_{4} \mid \mathcal{E}_{\mathbf{S}}^{c}\right] \leq \mathrm{E}_{\mathbf{S}_{1}, \mathbf{S}_{2}, \mathscr{C}_{2}}\left[\operatorname{Pr}_{\mathscr{C}_{1}, \mathscr{C}_{c}}\left(\left|\cos \varangle\left(\mathbf{w}_{2, P}, \mathbf{w}_{3}\right)\right|>\epsilon \mid\left(\mathbf{S}_{1}, \mathbf{S}_{2}, \mathbf{U}_{2}\right)=\left(\mathbf{s}_{1}, \mathbf{s}_{2}, \mathbf{u}_{2}\right), \mathcal{E}_{\mathbf{S}}^{c}\right)\right] .
$$

The desired upper bound now follows by noticing that conditioned on $\left(\mathbf{S}_{1}, \mathbf{S}_{2}, \mathbf{U}_{2}\right)=\left(\mathbf{s}_{1}, \mathbf{s}_{2}, \mathbf{u}_{2}\right)$, and $\mathscr{C}_{2}=\mathcal{C}_{2}$, the random vector $\mathbf{W}_{3} /\left\|\mathbf{W}_{3}\right\|$ is distributed uniformly on the surface of the centered $\mathbb{R}^{n-1}$-sphere of unit radius, that lies in the subspace that is orthogonal to $\mathbf{z}_{\mathrm{Q}_{1}}$. Hence, similarly as in the derivation for $\mathcal{A}_{2}$

$$
\operatorname{Pr}\left[\mathcal{A}_{4} \mid \mathcal{E}_{\mathbf{S}}^{c}\right] \leq \mathrm{E}_{\mathbf{S}_{1}, \mathbf{S}_{2}, \mathscr{C}_{2}}\left[\frac{2 C_{n-1}(\Theta)}{C_{n-1}(\pi)} \mid \mathcal{E}_{\mathbf{S}}^{c}\right]
$$

where $\Theta \triangleq \arccos (\epsilon)$. As $0<\epsilon \leq 1, \Theta \in\left(0, \frac{\pi}{2}\right)$, and thus by [11, Lemma B.4] the r.h.s. of (221) tends to 0 as $n \rightarrow \infty$, and therefore $\operatorname{Pr}\left[\mathcal{A}_{4} \mid \mathcal{E}_{\mathbf{S}}^{c}\right] \rightarrow 0$.

Combining Lemma 32 and Lemma 33 with (216) gives that for every $\delta>0$ and $0<\epsilon<0.3$ there exists an $n_{\mathcal{A}}^{\prime}(\delta, \epsilon)$ such that for all $n>n_{\mathcal{A}}^{\prime}(\delta, \epsilon)$

$$
\operatorname{Pr}\left[\mathcal{E}_{\left(\mathbf{X}_{2}, \mathbf{X}_{v}\right)} \cap \mathcal{E}_{\mathbf{X}_{2}}^{c} \cap \mathcal{E}_{\mathbf{X}_{v}}^{c} \cap \mathcal{E}_{\mathbf{S}}^{c}\right] \leq 3 \delta .
$$

\section{Proof of inequality (200):}

The error probability analysis can be outlined as follows:

1) The pair $\left(\mathbf{z}_{\mathrm{Q}_{1}}, \mathbf{s}_{2}\right) \notin A_{\epsilon}^{*(n)}$, where $A_{\epsilon}^{*(n)}$ denotes the $\epsilon$ - strongly jointly typical set of sequences (see [24, Chapter 2]). The probability of this event is small for large enough $n$, by the weak low of large numbers.

2) The sequence $\mathbf{z}_{\mathrm{Q}_{1}}$ is typical, but there does not exist a sequence $\mathbf{v} \in \mathcal{C}_{c}$ such that $\left(\mathbf{z}_{\mathrm{Q}_{1}}, \mathbf{v}\right) \in$ $A_{\epsilon}^{*(n)}$. As in the proof of the rate distortion theorem, the probability of this event is small 
if for $\epsilon^{\prime}<\epsilon$

$$
R_{\mathrm{c}}>I\left(V ; Z_{\mathrm{Q}_{1}}\right)+\delta\left(\epsilon^{\prime}\right)
$$

where $\delta\left(\epsilon^{\prime}\right) \rightarrow 0$ as $\epsilon^{\prime} \rightarrow 0$.

3) The pair $\left(\mathbf{z}_{\mathrm{Q}_{1}}, \mathbf{v}\right) \in A_{\epsilon}^{*(n)}$, but $\left(\mathbf{v}, \mathbf{s}_{2}\right) \notin A_{\epsilon}^{*(n)}$, i.e. the codeword is not jointly typical with the $\mathbf{s}_{2}$ sequence. By the Markov lemma [24, Lemma 12.1], the probability of this event is small if $n$ is large enough, since $V \multimap-Z_{Q_{1}} \multimap S_{2}$ forms a Markov chain.

4) There exists $\tilde{\mathbf{v}} \in \mathcal{C}_{c} \backslash \mathbf{v}^{*}$ within the same bin of $\mathbf{v}^{*}$, such that $\left(\tilde{\mathbf{v}}, \mathbf{s}_{2}\right) \in A_{\epsilon}^{*(n)}$. Since the probability that a randomly chosen $\tilde{\mathbf{v}}$ is jointly typical with $\mathbf{s}_{2}$ is $\approx 2^{-n\left[I\left(S_{2} ; V\right)-\delta(\epsilon)\right]}$, the probability of the former event is upper bounded by

$$
\begin{aligned}
\operatorname{Pr}\left(\exists \tilde{\mathbf{v}} \in \mathcal{C}_{c} \backslash \mathbf{v}^{*}:\left(\tilde{\mathbf{v}}, \mathbf{s}_{2}\right) \in A_{\epsilon}^{*(n)}\right) & \leq \frac{2^{n R_{c}}}{2^{n\left[R_{c}-I\left(S_{2} ; V\right)-\frac{1}{2} \delta(\epsilon)\right]}} 2^{-n\left[I\left(S_{2} ; V\right)-\delta(\epsilon)\right]} \\
& =\frac{2^{n\left[I\left(V ; Z_{Q_{1}}\right)+\delta\left(\epsilon^{\prime}\right)\right]}}{2^{n\left[I\left(V ; Z_{Q_{1}}\right)+\delta\left(\epsilon^{\prime}\right)-I\left(S_{2} ; V\right)-\frac{1}{2} \delta(\epsilon)\right]}} 2^{-n\left[I\left(S_{2} ; V\right)-\delta(\epsilon)\right]} \\
& =2^{-\frac{n}{2} \delta(\epsilon)},
\end{aligned}
$$

which goes to zero as $n \rightarrow \infty$.

The formal detailed proof is as follows: We start with a lemma that will be used to prove (200).

Lemma 34 Define the event that the quantized sequence $\mathbf{v}^{*}$ and the source sequence $\mathbf{s}_{2}$ have an atypical angle to each other

$$
\mathcal{E}_{\mathbf{v}, \mathbf{s}_{2}}=\left\{\left(\mathbf{s}_{1}, \mathbf{s}_{2}, \mathcal{C}_{1}, \mathcal{C}_{2}, \mathcal{C}_{c}\right):\left|\rho_{\mathbf{v}, \mathbf{s}_{2}}-\cos \varangle\left(\mathbf{v}^{*}\left(\mathbf{s}_{1}, \mathcal{C}_{1}\right), \mathbf{s}_{2}\right)\right|>5 \epsilon\right\} .
$$

Then, for every $\delta>0$ and $\epsilon>0$ there exists an $n^{\prime}(\delta, \epsilon) \in \mathbb{N}$ such that for all $n>n^{\prime}(\delta, \epsilon)$

$$
\operatorname{Pr}\left[\mathcal{E}_{\mathbf{v}, \mathbf{s}_{2}} \cap \mathcal{E}_{\mathbf{X}_{v}}^{c} \cap \mathcal{E}_{\mathbf{S}}^{c}\right]<\delta .
$$

Proof: We start with the following decomposition

$$
\begin{aligned}
\cos \varangle\left(\mathbf{v}^{*}, \mathbf{s}_{2}\right) & =\frac{\left\langle\mathbf{v}^{*}, \mathbf{s}_{2}\right\rangle}{\left\|\mathbf{v}^{*}\right\|\left\|\mathbf{s}_{2}\right\|} \\
& \stackrel{(a)}{=} \frac{\left\langle\mathbf{v}^{*}, \rho \mathbf{s}_{1}+\mathbf{z}_{\mathrm{G}_{2}}\right\rangle}{\left\|\mathbf{v}^{*}\right\|\left\|\mathbf{s}_{2}\right\|} \\
& =\frac{\rho\left\langle\mathbf{v}^{*}, \mathbf{s}_{1}\right\rangle+\left\langle\mathbf{v}^{*}, \mathbf{z}_{\mathrm{G}_{2}}\right\rangle}{\left\|\mathbf{v}^{*}\right\|\left\|\mathbf{s}_{2}\right\|},
\end{aligned}
$$

where in (a) we represent $\mathbf{s}_{2}$ as a scaled version of $\mathbf{s}_{1}$ corrupted by an additive gaussian noise $\mathbf{z}_{G 2}$. More precisely,

$$
\mathbf{s}_{2}=\rho \mathbf{s}_{1}+\mathbf{z}_{\mathrm{G}_{2}} \quad \text { where } \quad \rho=\frac{\left\|\mathbf{s}_{2}\right\|}{\left\|\mathbf{s}_{1}\right\|} \cos \varangle\left(\mathbf{s}_{1}, \mathbf{s}_{2}\right) .
$$

With this choice of $\rho$, the vector $\mathbf{z}_{\mathrm{G} 2}$ is always orthogonal to $\mathbf{s}_{1}$.

Now, define the two events

$$
\begin{aligned}
& \mathcal{A}_{1}=\left\{\left(\mathbf{s}_{1}, \mathbf{s}_{2}, \mathcal{C}_{1}, \mathcal{C}_{2}, \mathcal{C}_{c}\right):\left|\rho_{\mathbf{v}, \mathbf{s}_{2}}-\frac{\rho}{\left\|\mathbf{v}^{*}\right\|\left\|\mathbf{s}_{2}\right\|}\left\langle\mathbf{v}^{*}, \mathbf{s}_{1}\right\rangle\right|>4 \epsilon\right\} \\
& \mathcal{A}_{2}=\left\{\left(\mathbf{s}_{1}, \mathbf{s}_{2}, \mathcal{C}_{1}, \mathcal{C}_{2}, \mathcal{C}_{c}\right):\left|\frac{1}{\left\|\mathbf{v}^{*}\right\|\left\|\mathbf{s}_{2}\right\|}\left\langle\mathbf{v}^{*}, \mathbf{z}_{\mathrm{G}_{2}}\right\rangle\right|>\epsilon\right\} .
\end{aligned}
$$


Note that by (222), $\mathcal{E}_{\mathbf{v}, \mathbf{s}_{2}}=\left\{\left(\mathbf{s}_{1}, \mathbf{s}_{2}, \mathcal{C}_{1}, \mathcal{C}_{2}, \mathcal{C}_{c}\right):\left|\rho_{\mathbf{v}, \mathbf{s}_{2}}-\cos \varangle\left(\mathbf{v}^{*}, \mathbf{s}_{2}\right)\right|>4 \epsilon\right\} \subset\left(\mathcal{A}_{1} \cup \mathcal{A}_{2}\right)$. Thus,

$$
\begin{aligned}
\operatorname{Pr}\left[\mathcal{E}_{\mathbf{v}, \mathbf{S}_{2}} \cap \mathcal{E}_{\mathbf{X}_{v}}^{c} \cap \mathcal{E}_{\mathbf{S}}^{c}\right] & \leq \operatorname{Pr}\left[\mathcal{A}_{1} \cap \mathcal{E}_{\mathbf{X}_{v}}^{c} \cap \mathcal{E}_{\mathbf{S}}^{c}\right]+\operatorname{Pr}\left[\mathcal{A}_{2} \cap \mathcal{E}_{\mathbf{X}_{v}}^{c} \cap \mathcal{E}_{\mathbf{S}}^{c}\right] \\
& \leq \operatorname{Pr}\left[\mathcal{A}_{1} \mid \mathcal{E}_{\mathbf{X}_{v}}^{c} \cap \mathcal{E}_{\mathbf{S}}^{c}\right]+\operatorname{Pr}\left[\mathcal{A}_{2} \mid \mathcal{E}_{\mathbf{S}}^{c}\right] .
\end{aligned}
$$

The two terms on the r.h.s. of (224) are now bounded in the following two lemmas.

Lemma 35 For $\epsilon<1$

$$
\operatorname{Pr}\left[\mathcal{A}_{1} \mid \mathcal{E}_{\mathbf{S}}^{c} \cap \mathcal{E}_{\mathbf{X}_{v}}^{c}\right]=0 .
$$

Proof: We first note that the term in the definition of $\mathcal{A}_{1}$ can be rewritten as

$$
\frac{\rho}{\left\|\mathbf{v}^{*}\right\|\left\|\mathbf{s}_{2}\right\|}\left\langle\mathbf{v}^{*}, \mathbf{s}_{1}\right\rangle=\cos \varangle\left(\mathbf{s}_{1}, \mathbf{s}_{2}\right) \cos \varangle\left(\mathbf{v}^{*}, \mathbf{s}_{1}\right) .
$$

Note that the second term satisfies

$$
\cos \varangle\left(\mathbf{v}^{*}, \mathbf{s}_{1}\right)=\frac{\left\langle\mathbf{v}^{*}, \mathbf{s}_{1}\right\rangle}{\left\|\mathbf{v}^{*}\right\|\left\|\mathbf{s}_{1}\right\|}=\frac{\left\langle\mathbf{v}^{*}, \mathbf{u}_{1}^{*}+\mathbf{z}_{\mathrm{Q}_{1}}\right\rangle}{\left\|\mathbf{v}^{*}\right\|\left\|\mathbf{s}_{1}\right\|}=\frac{\left\langle\mathbf{v}^{*}, \mathbf{u}_{1}^{*}\right\rangle}{\left\|\mathbf{v}^{*}\right\|\left\|\mathbf{s}_{1}\right\|}+\frac{\left\langle\mathbf{v}^{*}, \mathbf{z}_{\mathrm{Q}_{1}}\right\rangle}{\left\|\mathbf{v}^{*}\right\|\left\|\mathbf{s}_{1}\right\|} .
$$

By 239 and Lemma 42, the first term can be bounded by

$$
\left|\frac{\left\langle\mathbf{v}^{*}, \mathbf{u}_{1}^{*}\right\rangle}{\left\|\mathbf{v}^{*}\right\|\left\|\mathbf{s}_{1}\right\|}\right| \leq \frac{12 \delta+3 \epsilon}{\sqrt{2^{-2 R_{1}}\left(1-2^{-2 R_{\mathrm{c}}}\right)}}=\epsilon_{1} .
$$

The second term can be factorized

$$
\frac{\left\langle\mathbf{v}^{*}, \mathbf{z}_{\mathrm{Q}_{1}}\right\rangle}{\left\|\mathbf{v}^{*}\right\|\left\|\mathbf{s}_{1}\right\|}=\frac{\left\langle\mathbf{v}^{*}, \mathbf{z}_{\mathrm{Q}_{1}}\right\rangle}{\left\|\mathbf{v}^{*}\right\|\left\|\mathbf{z}_{\mathrm{Q}_{1}}\right\|} \frac{\left\|\mathbf{z}_{\mathrm{Q}_{1}}\right\|}{\left\|\mathbf{s}_{1}\right\|}=\cos \varangle\left(\mathbf{z}_{\mathrm{Q}_{1}}, \mathbf{v}^{*}\right) \sqrt{2^{-2 R_{1}}} .
$$

We can now upper and lower bound the r.h.s. of (225) for $\left(\mathbf{s}_{1}, \mathbf{s}_{2}, \mathcal{C}_{1}, \mathcal{C}_{2}, \mathcal{C}_{c}\right) \in \mathcal{E}_{\mathbf{S}}^{c} \cap \mathcal{E}_{\mathbf{X}_{v}}^{c}$ by noticing that $\left(\mathbf{s}_{1}, \mathbf{s}_{2}, \mathcal{C}_{1}, \mathcal{C}_{2}, \mathcal{C}_{c}\right) \in \mathcal{E}_{\mathbf{S}}^{c}$ implies

$$
\left|\cos \varangle\left(\mathbf{s}_{1}, \mathbf{s}_{2}\right)-\rho\right|<\rho \epsilon,
$$

and that $\left(\mathbf{s}_{1}, \mathbf{s}_{2}, \mathcal{C}_{1}, \mathcal{C}_{2}, \mathcal{C}_{c}\right) \in \mathcal{E}_{\mathbf{X}_{v}}^{c}$ implies

$$
\left|\sqrt{1-2^{-2 R_{\mathrm{c}}}}-\cos \varangle\left(\mathbf{z}_{\mathrm{Q}_{1}}, \mathbf{v}^{*}\right)\right|<\epsilon \sqrt{1-2^{-2 R_{\mathrm{c}}}} .
$$

Hence, combined with 225) this gives

$$
\rho_{\mathbf{v}, \mathbf{s}_{2}}(1-\epsilon)^{2}+\rho \epsilon_{1}(1-\epsilon) \leq \frac{\rho}{\left\|\mathbf{v}^{*}\right\|\left\|\mathbf{s}_{2}\right\|}\left\langle\mathbf{v}^{*}, \mathbf{s}_{1}\right\rangle \leq \rho_{\mathbf{v}, \mathbf{s}_{2}}(1+\epsilon)^{2}+\rho \epsilon_{1}(1+\epsilon),
$$

whenever $\left(\mathbf{s}_{1}, \mathbf{s}_{2}, \mathcal{C}_{1}, \mathcal{C}_{2}, \mathcal{C}_{c}\right) \in \mathcal{E}_{\mathbf{S}}^{c} \cap \mathcal{E}_{\mathbf{X}_{v}}^{c}$. The 1.h.s. can be lower bounded by $(1-2 \epsilon) \leq(1-\epsilon)^{2}$, and the r.h.s. can be upper bounded by $(1+\epsilon)^{2} \leq(1+3 \epsilon)$ whenever $\epsilon \leq 1$. Hence, for $\epsilon \leq 1$

$$
\left|\rho_{\mathbf{v}, \mathbf{s}_{2}}-\frac{\rho}{\left\|\mathbf{v}^{*}\right\|\left\|\mathbf{s}_{1}\right\|}\left\langle\mathbf{v}^{*}, \mathbf{s}_{2}\right\rangle\right| \leq 3 \rho_{\mathbf{v}, \mathbf{s}_{2}} \epsilon+\rho \epsilon_{1}(1+\epsilon) \leq 4 \epsilon
$$

and thus

$$
\operatorname{Pr}\left[\mathcal{A}_{1} \mid \mathcal{E}_{\mathbf{S}}^{c} \cap \mathcal{E}_{\mathbf{X}_{v}}^{c}\right]=0
$$

Lemma 36 For every $\delta>0$ and $\epsilon>0$ there exists an $n_{\mathcal{A}}^{\prime}(\delta, \epsilon)$ such that for all $n>n_{\mathcal{A}}^{\prime}(\delta, \epsilon)$

$$
\operatorname{Pr}\left[\mathcal{A}_{2} \mid \mathcal{E}_{\mathbf{S}}^{c}\right]<\delta
$$


Proof: By similar arguments as in proof of Lemma 29, it follows that for every $\epsilon>0$, $\operatorname{Pr}\left[\mathcal{A}_{2} \mid \mathcal{E}_{\mathbf{S}}^{c}\right] \rightarrow 0$ as $n \rightarrow \infty$.

Combining Lemma 35 and Lemma 36 with (224) gives that for every $\delta>0$ and $0<\epsilon<1$ there exists an $n_{\mathcal{A}}^{\prime}(\delta, \epsilon)$ such that for all $n>n_{\mathcal{A}}^{\prime}(\delta, \epsilon)$

$$
\operatorname{Pr}\left[\mathcal{E}_{\mathbf{v}, \mathbf{s}_{2}} \cap \mathcal{E}_{\mathbf{X}_{v}}^{c} \cap \mathcal{E}_{\mathbf{S}}^{c}\right] \leq \delta .
$$

We now start with a definition that will be used to prove (200).

$$
\mathcal{E}_{\mathbf{X}_{\mathrm{WZ}}}^{\prime} \triangleq\left\{\left(\mathbf{s}_{1}, \mathbf{s}_{2}, \mathcal{C}_{1}, \mathcal{C}_{2}, \mathcal{C}_{c}\right): \exists \tilde{\mathbf{v}} \in \mathcal{C}_{c} \backslash\left\{\mathbf{v}^{*}\right\} \text { s.t. } \cos \varangle\left(\tilde{\mathbf{v}}, \mathbf{s}_{2}\right) \geq \rho_{\mathbf{v}, \mathbf{s}_{2}}-5 \epsilon\right\} .
$$

Note that

$$
\mathcal{E}_{\mathbf{X}_{\mathrm{WZ}}}=\left\{\left(\mathbf{s}_{1}, \mathbf{s}_{2}, \mathcal{C}_{1}, \mathcal{C}_{2}, \mathcal{C}_{c}\right): \exists \tilde{\mathbf{v}} \in \mathcal{C}_{c} \backslash\left\{\mathbf{v}^{*}\right\} \text { s.t. }\left|\rho_{\mathbf{v}, \mathbf{s}_{2}}-\cos \varangle\left(\tilde{\mathbf{v}}, \mathbf{s}_{2}\right)\right| \leq 5 \epsilon\right\} \subseteq \mathcal{E}_{\mathbf{X}_{\mathrm{wZ}}}^{\prime} .
$$

We now state one more lemma that will be used for the proof of 200):

Lemma 37 For every $\Delta \in(0,1]$, let the set $\mathcal{G}$ be given by

$$
\mathcal{G}=\left\{\left(\mathbf{s}_{1}, \mathbf{s}_{2}, \mathcal{C}_{1}, \mathcal{C}_{2}, \mathcal{C}_{c}\right): \exists \mathbf{v} \in \mathcal{C}_{c} \backslash\left\{\mathbf{v}^{*}\right\} \text { s.t. } \cos \varangle\left(\mathbf{s}_{2}, \mathbf{v}\right) \geq \Delta\right\} .
$$

Then,

$$
\frac{1}{n} \log M_{b}<-\frac{1}{2} \log \left(1-\Delta^{2}\right) \quad \Longrightarrow \quad\left(\lim _{n \rightarrow \infty} \operatorname{Pr}\left[\mathcal{G} \mid \mathcal{E}_{\mathbf{X}_{v}}^{c}\right]=0, \epsilon>0\right),
$$

where $M_{b}$ denotes the bin size in the partitioned codebook $\mathcal{C}_{c}$, and $\mathcal{E}_{\mathbf{X}_{v}}$ is defined in (96).

Proof: The proof follows from upper-bounding in every point on $\mathcal{S}_{c}$ the density of every $\mathbf{v} \in \mathcal{C}_{c} \backslash\left\{\mathbf{v}^{*}\right\}$ and then using a standard argument from sphere-packing.

Next,

$$
\operatorname{Pr}\left[\mathcal{E}_{\mathbf{X}_{\mathrm{WZ}}} \cap \mathcal{E}_{\mathbf{X}_{v}}^{c} \cap \mathcal{E}_{\mathbf{S}}^{c}\right] \stackrel{(a)}{\leq} \operatorname{Pr}\left[\mathcal{E}_{\mathbf{X}_{\mathrm{WZ}}}^{\prime} \cap \mathcal{E}_{\mathbf{X}_{v}}^{c} \cap \mathcal{E}_{\mathbf{S}}^{c}\right] \stackrel{(b)}{\leq} \operatorname{Pr}\left[\mathcal{E}_{\mathbf{X}_{\mathrm{WZ}}}^{\prime} \mid \mathcal{E}_{\mathbf{X}_{v}}^{c}\right],
$$

where (a) follows by (228) and (b) follows because $\mathcal{E}_{\mathbf{X}}^{c} \subseteq \mathcal{E}_{\mathbf{X}}^{c}$.

The proof of 200) is now completed by combining (228) with Lemma 37 This gives that for every $\delta>0$ and every $\epsilon>0$ there exists some $n^{\prime}(\delta, \epsilon)$ such that for all $n>n^{\prime}(\delta, \epsilon)$, we have

$$
\operatorname{Pr}\left[\mathcal{E}_{\mathbf{X}_{\mathrm{wZ}}} \cap \mathcal{E}_{\mathbf{X}_{v}}^{c} \cap \mathcal{E}_{\mathbf{S}}^{c}\right]<\delta,
$$

whenever

$$
\frac{1}{n} \log M_{b}<-\frac{1}{2} \log \left(1-\left(\rho_{\mathbf{v}, \mathbf{s}_{2}}-5 \epsilon\right)^{2}\right) .
$$

The constraint (229) yields the following bound on the bin size

$$
M_{b} \leq\left(1-\rho_{\mathbf{v}, \mathbf{s}_{2}}^{2}\right)^{-\frac{n}{2}} 2^{-n \delta(\epsilon)},
$$

where $\delta(\epsilon) \rightarrow 0$ as $\epsilon \rightarrow 0$.

The desired result follows now by noticing that the bin size in our code construction (defined in (56)) satisfies (230).

\section{Upper bound on expected distortion - Proof of Proposition 5}

We derive an upper bound on the achievable distortion for the proposed vector-quantizer scheme. By Corollary 4 it suffices to analyze the genie-aided scheme. Since $\hat{\mathbf{S}}_{1}^{\mathrm{G}}=\gamma_{1,1} \mathbf{U}_{1}{ }^{*}+$ 
$\gamma_{1,2} \mathbf{U}_{2}{ }^{*}+\gamma_{1,3} \mathbf{V}^{*}$, we have

$$
\begin{aligned}
D_{1}= & \frac{1}{n} \mathrm{E}\left[\left\|\mathbf{S}_{1}-\hat{\mathbf{S}}_{1}^{\mathrm{G}}\right\|^{2}\right] \\
= & \frac{1}{n} \mathrm{E}\left[\left\|\mathbf{S}_{1}-\left(\gamma_{1,1} \mathbf{U}_{1}{ }^{*}+\gamma_{1,2} \mathbf{U}_{2}{ }^{*}+\gamma_{1,3} \mathbf{V}^{*}\right)\right\|^{2}\right] \\
= & \frac{1}{n}\left(\mathrm{E}\left[\left\|\mathbf{S}_{1}\right\|^{2}\right]-2 \gamma_{1,1} \mathrm{E}\left[\left\langle\mathbf{S}_{1}, \mathbf{U}_{1}{ }^{*}\right\rangle\right]-2 \gamma_{1,2} \mathrm{E}\left[\left\langle\mathbf{S}_{1}, \mathbf{U}_{2}{ }^{*}\right\rangle\right]-2 \gamma_{1,3} \mathrm{E}\left[\left\langle\mathbf{S}_{1}, \mathbf{V}^{*}\right\rangle\right]\right. \\
& +\gamma_{1,1}{ }^{2} \mathrm{E}\left[\left\|\mathbf{U}_{1}{ }^{*}\right\|^{2}\right]+2 \gamma_{1,1} \gamma_{1,2} \mathrm{E}\left[\left\langle\mathbf{U}_{1}{ }^{*}, \mathbf{U}_{2}{ }^{*}\right\rangle\right]+\gamma_{1,2}{ }^{2} \mathrm{E}\left[\left\|\mathbf{U}_{2}{ }^{*}\right\|^{2}\right] \\
& \left.+2 \gamma_{1,1} \gamma_{1,3} \mathrm{E}\left[\left\langle\mathbf{U}_{1}{ }^{*}, \mathbf{V}^{*}\right\rangle\right]+2 \gamma_{1,2} \gamma_{1,3} \mathrm{E}\left[\left\langle\mathbf{U}_{2}{ }^{*}, \mathbf{V}^{*}\right\rangle\right]+\gamma_{1,3}{ }^{2} \mathrm{E}\left[\left\|\mathbf{V}^{*}\right\|^{2}\right]\right) \\
= & \sigma^{2}-2 \gamma_{1,1} \frac{1}{n} \mathrm{E}\left[\left\langle\mathbf{S}_{1}, \mathbf{U}_{1}{ }^{*}\right\rangle\right]-2 \gamma_{1,2} \frac{1}{n} \mathrm{E}\left[\left\langle\mathbf{S}_{1}, \mathbf{U}_{2}{ }^{*}\right\rangle\right]-2 \gamma_{1,3} \frac{1}{n} \mathrm{E}\left[\left\langle\mathbf{S}_{1}, \mathbf{V}^{*}\right\rangle\right] \\
& +\gamma_{1,1}{ }^{2}\left(\sigma^{2}\left(1-2^{-2 R_{1}}\right)\right)+2 \gamma_{1,1} \gamma_{1,2} \frac{1}{n} \mathrm{E}\left[\left\langle\mathbf{U}_{1}{ }^{*}, \mathbf{U}_{2}{ }^{*}\right\rangle\right]+\gamma_{1,2}{ }^{2}\left(\sigma^{2}\left(1-2^{-2 R_{2}}\right)\right) \\
& +2 \gamma_{1,1} \gamma_{1,3} \frac{1}{n} \mathrm{E}\left[\left\langle\mathbf{U}_{1}{ }^{*}, \mathbf{V}^{*}\right\rangle\right]+2 \gamma_{1,2} \gamma_{1,3} \frac{1}{n} \mathrm{E}\left[\left\langle\mathbf{U}_{2}{ }^{*}, \mathbf{V}^{*}\right\rangle\right]+\gamma_{1,3}{ }^{2}\left(\sigma^{2} 2^{-2 R_{1}}\left(1-2^{-2 R_{\mathrm{c}}}\right)\right),
\end{aligned}
$$

where in the last equality all expected squared norms have been replaced by their explicit values, i.e. $\mathrm{E}\left[\left\|\mathbf{S}_{1}\right\|^{2}\right]=n \sigma^{2}$, and $\mathrm{E}\left[\left\|\mathbf{U}_{i}\right\|^{2}\right]=n \sigma^{2}\left(1-2^{-2 R_{i}}\right)$ for $i \in\{1,2\}$ and $\mathrm{E}\left[\|\mathbf{V}\|^{2}\right]=$ $n \sigma^{2} 2^{-2 R_{1}}\left(1-2^{-2 R_{\mathrm{c}}}\right)$. The remaining expectations of the inner products are bounded in the following six lemmas.

Lemma 38 For every $\delta>0$ and $0.3>\epsilon>0$ and every positive integer $n$

$$
\frac{1}{n} \mathrm{E}\left[\left\langle\mathbf{S}_{1}, \mathbf{U}_{1}^{*}\right\rangle\right] \geq \sigma^{2}\left(1-2^{-2 R_{1}}\right)(1-2 \epsilon)(1-13 \delta) .
$$

Proof:

$$
\begin{aligned}
\frac{1}{n} \mathrm{E}\left[\left\langle\mathbf{S}_{1}, \mathbf{U}_{1}{ }^{*}\right\rangle\right]= & \frac{1}{n} \underbrace{\mathrm{E}\left[\left\|\mathbf{S}_{1}\right\|\left\|\mathbf{U}_{1}{ }^{*}\right\| \cos \varangle\left(\mathbf{S}_{1}, \mathbf{U}_{1}{ }^{*}\right) \mid \mathcal{E}_{\mathbf{S}} \cup \mathcal{E}_{\mathbf{X}}\right]}_{\geq 0} \operatorname{Pr}\left[\mathcal{E}_{\mathbf{S}} \cup \mathcal{E}_{\mathbf{X}}\right] \\
& +\frac{1}{n} \mathrm{E}\left[\left\|\mathbf{S}_{1}\right\|\left\|\mathbf{U}_{1}{ }^{*}\right\| \cos \varangle\left(\mathbf{S}_{1}, \mathbf{U}_{1}{ }^{*}\right) \mid \mathcal{E}_{\mathbf{S}}^{c} \cap \mathcal{E}_{\mathbf{X}}^{c}\right] \operatorname{Pr}\left[\mathcal{E}_{\mathbf{S}}^{c} \cap \mathcal{E}_{\mathbf{X}}^{c}\right] \\
\geq & \frac{1}{n} \mathrm{E}\left[\left\|\mathbf{S}_{1}\right\|\left\|\mathbf{U}_{1}{ }^{*}\right\| \cos \varangle\left(\mathbf{S}_{1}, \mathbf{U}_{1}{ }^{*}\right) \mid \mathcal{E}_{\mathbf{S}}^{c} \cap \mathcal{E}_{\mathbf{X}}^{c}\right] \operatorname{Pr}\left[\mathcal{E}_{\mathbf{S}}^{c} \cap \mathcal{E}_{\mathbf{X}}^{c}\right] \\
\geq & \sqrt{\sigma^{2}(1-\epsilon) \sigma^{2}\left(1-2^{-2 R_{1}}\right)\left(1-2^{-2 R_{1}}\right)}(1-\epsilon) \operatorname{Pr}\left[\mathcal{E}_{\mathbf{S}}^{c} \cap \mathcal{E}_{\mathbf{X}}^{c}\right] \\
\geq & \sigma^{2}\left(1-2^{-2 R_{1}}\right)(1-\epsilon)^{2}\left(1-\operatorname{Pr}\left[\mathcal{E}_{\mathbf{S}} \cup \mathcal{E}_{\mathbf{X}}\right]\right) \\
\geq & \sigma^{2}\left(1-2^{-2 R_{1}}\right)(1-2 \epsilon)\left(1-\operatorname{Pr}\left[\mathcal{E}_{\mathbf{S}}\right]-\operatorname{Pr}\left[\mathcal{E}_{\mathbf{X}}\right]\right),
\end{aligned}
$$

where in the first equality the first expectation term is non-negative because if $\mathbf{U}_{1}^{*}=0$, then it is equal to zero, and if $\mathbf{U}_{1}^{*} \neq 0$, then by the conditioning on $\mathcal{E}_{\mathbf{X}}$ it follows that $\cos \varangle\left(\mathbf{S}_{1}, \mathbf{U}_{1}{ }^{*}\right)>0$.

By Lemma 6 and Lemma 8 it now follows that for every $\delta>0$ and $0.3>\epsilon>0$ there exists an $n^{\prime}(\delta, \epsilon) \in \mathbb{N}$ such that for all $n>n^{\prime}(\delta, \epsilon)$

$$
\frac{1}{n} \mathrm{E}\left[\left\langle\mathbf{S}_{1}, \mathbf{U}_{1}{ }^{*}\right\rangle\right] \geq \sigma^{2}\left(1-2^{-2 R_{1}}\right)(1-2 \epsilon)(1-13 \delta) .
$$


Lemma 39 For every $\delta>0$ and $0.3>\epsilon>0$, there exists an $n^{\prime}(\delta, \epsilon) \in \mathbb{N}$ such that for all $n>n^{\prime}(\delta, \epsilon)$

$$
\frac{1}{n} \mathrm{E}\left[\left\langle\mathbf{U}_{1}^{*}, \mathbf{U}_{2}^{*}\right\rangle\right] \leq \sigma^{2} 12 \delta+\rho \sigma^{2}\left(1-2^{-2 R_{1}}\right)\left(1-2^{-2 R_{2}}\right)(1+7 \epsilon) .
$$

Proof:

$$
\begin{aligned}
\frac{1}{n} \mathrm{E}\left[\left\langle\mathbf{U}_{1}{ }^{*}, \mathbf{U}_{2}{ }^{*}\right\rangle\right]= & \frac{1}{n} \mathrm{E}\left[\left\langle\mathbf{U}_{1}^{*}, \mathbf{U}_{2}^{*}\right\rangle \mid \mathcal{E}_{\mathbf{X}}\right] \operatorname{Pr}\left[\mathcal{E}_{\mathbf{X}}\right]+\frac{1}{n} \mathrm{E}\left[\left\langle\mathbf{U}_{1}^{*}, \mathbf{U}_{2}^{*}\right\rangle \mid \mathcal{E}_{\mathbf{X}}^{c}\right] \operatorname{Pr}\left[\mathcal{E}_{\mathbf{X}}^{c}\right] \\
\leq & \frac{1}{n} \mathrm{E}\left[\left\|\mathbf{U}_{1}^{*}\right\|\left\|\mathbf{U}_{2}^{*}\right\| \mid \mathcal{E}_{\mathbf{X}}\right] \operatorname{Pr}\left[\mathcal{E}_{\mathbf{X}}\right]+\frac{1}{n} \mathrm{E}\left[\left\|\mathbf{U}_{1}^{*}\right\|\left\|\mathbf{U}_{2}^{*}\right\|\left|\cos \varangle\left(\mathbf{U}_{1}^{*}, \mathbf{U}_{2}^{*}\right)\right| \mathcal{E}_{\mathbf{X}}{ }^{c}\right] \\
\leq & \frac{1}{n} \sqrt{n \sigma^{2}\left(1-2^{-2 R_{1}}\right)} \sqrt{n \sigma^{2}\left(1-2^{-2 R_{2}}\right)} \operatorname{Pr}\left[\mathcal{E}_{\mathbf{X}}\right] \\
& +\frac{1}{n} \sqrt{n \sigma^{2}\left(1-2^{-2 R_{1}}\right)} \sqrt{n \sigma^{2}\left(1-2^{-2 R_{2}}\right)} \tilde{\rho}(1+7 \epsilon) \\
\leq & \sigma^{2} \operatorname{Pr}\left[\mathcal{E}_{\mathbf{X}}\right]+\rho \sigma^{2}\left(1-2^{-2 R_{1}}\right)\left(1-2^{-2 R_{2}}\right)(1+7 \epsilon) .
\end{aligned}
$$

Thus, it follows by Lemma 8 that for every $\delta>0$ and $0.3>\epsilon>0$ there exists an $n^{\prime}(\delta, \epsilon) \in \mathbb{N}$ such that for all $n>n^{\prime}(\delta, \epsilon)$

$$
\frac{1}{n} \mathrm{E}\left[\left\langle\mathbf{U}_{1}^{*}, \mathbf{U}_{2}^{*}\right\rangle\right] \leq \sigma^{2} 12 \delta+\rho \sigma^{2}\left(1-2^{-2 R_{1}}\right)\left(1-2^{-2 R_{2}}\right)(1+7 \epsilon) .
$$

Lemma 40 For every $\delta>0$ and $0.3>\epsilon>0$ there exists an $n^{\prime}(\delta, \epsilon) \in \mathbb{N}$ such that for all $n>n^{\prime}(\delta, \epsilon)$

$$
\frac{1}{n} \mathrm{E}\left[\left\langle\mathbf{S}_{1}^{*}, \mathbf{U}_{2}^{*}\right\rangle\right] \geq \rho \sigma^{2}\left(1-2^{-2 R_{2}}\right)(1-\epsilon)^{3}-\sigma^{2}(\epsilon+39 \delta+12 \delta \epsilon) .
$$

Proof: We begin with the following decomposition:

$$
\begin{aligned}
\frac{1}{n} \mathrm{E}\left[\left\langle\mathbf{S}_{1}, \mathbf{U}_{2}^{*}\right\rangle\right]= & \frac{1}{n} \mathrm{E}\left[\left\langle\mathbf{S}_{1}, \mathbf{U}_{2}^{*}\right\rangle \mid \mathcal{E}_{\mathbf{S}} \cup \mathcal{E}_{\mathbf{X}_{2}}\right] \operatorname{Pr}\left[\mathcal{E}_{\mathbf{S}} \cup \mathcal{E}_{\mathbf{X}_{2}}\right] \\
& +\frac{1}{n} \mathrm{E}\left[\left\langle\mathbf{S}_{1}, \mathbf{U}_{2}{ }^{*}\right\rangle \mid \mathcal{E}_{\mathbf{S}}^{c} \cap \mathcal{E}_{\mathbf{X}_{2}}^{c}\right] \operatorname{Pr}\left[\mathcal{E}_{\mathbf{S}}^{c} \cap \mathcal{E}_{\mathbf{X}_{2}}^{c}\right] .
\end{aligned}
$$

The first term on the r.h.s. of (232) is lower bounded as follows:

$$
\begin{aligned}
& \frac{1}{n} \mathrm{E}\left[\left\langle\mathbf{S}_{1}, \mathbf{U}_{2}{ }^{*}\right\rangle \mid \mathcal{E}_{\mathbf{S}} \cup \mathcal{E}_{\mathbf{X}_{2}}\right] \operatorname{Pr}\left[\mathcal{E}_{\mathbf{S}} \cup \mathcal{E}_{\mathbf{X}_{2}}\right] \\
& \stackrel{(a)}{\geq}-\frac{1}{n} \mathrm{E}\left[\left\|\mathbf{S}_{1}\right\|^{2}+\left\|\mathbf{U}_{2}{ }^{*}\right\|^{2} \mid \mathcal{E}_{\mathbf{S}} \cup \mathcal{E}_{\mathbf{X}_{2}}\right] \operatorname{Pr}\left(\mathcal{E}_{\mathbf{S}} \cup \mathcal{E}_{\mathbf{X}_{2}}\right) \\
& \stackrel{(b)}{\geq}-\frac{1}{n}\left(\mathrm{E}\left[\left\|\mathbf{S}_{1}\right\|^{2} \mid \mathcal{E}_{\mathbf{S}}\right] \operatorname{Pr}\left[\mathcal{E}_{\mathbf{S}}\right]+\mathrm{E}\left[\left\|\mathbf{S}_{1}\right\|^{2} \mid \mathcal{E}_{\mathbf{S}}^{c} \cap \mathcal{E}_{\mathbf{X}_{2}}\right] \operatorname{Pr}\left[\mathcal{E}_{\mathbf{S}}^{c} \cap \mathcal{E}_{\mathbf{X}}\right]\right. \\
& \left.\quad+\left\|\mathbf{U}_{2}{ }^{*}\right\|^{2}\left(\operatorname{Pr}\left[\mathcal{E}_{\mathbf{S}}\right]+\operatorname{Pr}\left[\mathcal{E}_{\mathbf{X}}\right]\right)\right) \\
& \stackrel{(c)}{\geq}-\left(\sigma^{2}\left(\epsilon+\operatorname{Pr}\left[\mathcal{E}_{\mathbf{S}}\right]\right)+\sigma^{2}(1+\epsilon) \operatorname{Pr}\left[\mathcal{E}_{\mathbf{X}}\right]+\sigma^{2}\left(1-2^{-2 R_{2}}\right)\left(\operatorname{Pr}\left[\mathcal{E}_{\mathbf{S}}\right]+\operatorname{Pr}\left[\mathcal{E}_{\mathbf{X}}\right]\right)\right) \\
& \geq-\sigma^{2}\left(\epsilon+2 \operatorname{Pr}\left[\mathcal{E}_{\mathbf{S}}\right]+(2+\epsilon) \operatorname{Pr}\left[\mathcal{E}_{\mathbf{X}}\right]\right),
\end{aligned}
$$

where in (a) we have used that for any two vectors $\mathbf{v} \in \mathbb{R}^{n}$ and $\mathbf{w} \in \mathbb{R}^{n}$

$$
\mid\langle\mathbf{v}, \mathbf{w}\rangle\left\|\leq \frac{1}{2}\left(\|\mathbf{v}\|^{2}+\|\mathbf{w}\|^{2}\right) \leq\right\| \mathbf{v}\left\|^{2}+\right\| \mathbf{w} \|^{2},
$$

in (b) we have used that $\mathcal{E}_{\mathbf{X}} \supseteq \mathcal{E}_{\mathbf{X}_{2}}$, and in (c) we have used Lemma 10 
We now turn to lower bounding the second term on the r.h.s. of (232). The probability term is lower bounded as follows:

$$
\begin{aligned}
\operatorname{Pr}\left[\mathcal{E}_{\mathbf{S}}^{c} \cap \mathcal{E}_{\mathbf{X}_{2}}^{c}\right] & =1-\operatorname{Pr}\left[\mathcal{E}_{\mathbf{S}} \cup \mathcal{E}_{\mathbf{X}_{2}}\right] \\
& \geq 1-\left(\operatorname{Pr}\left[\mathcal{E}_{\mathbf{S}}\right]+\operatorname{Pr}\left[\mathcal{E}_{\mathbf{X}}\right]\right) .
\end{aligned}
$$

To lower bound the expectation term, we represent $\mathbf{u}_{i}^{*}$ as a scaled version of $\mathbf{s}_{i}$ corrupted by an additive "quantization noise" $\mathbf{w}_{i}^{*}$. More precisely,

$$
\mathbf{u}_{i}^{*}=\nu_{i} \mathbf{s}_{i}+\mathbf{w}_{i} \quad \text { where } \quad \nu_{i}=\frac{\left\|\mathbf{u}_{i}^{*}\right\|}{\left\|\mathbf{s}_{i}^{*}\right\|} \cos \varangle\left(\mathbf{s}_{i}, \mathbf{u}_{i}^{*}\right), i \in\{1,2\} .
$$

With this choice of $\nu_{i}$, the vector $\mathbf{w}_{i}$ is always orthogonal to $\mathbf{s}_{i}$. By (236), the inner product $\left\langle\mathbf{S}_{1}, \mathbf{U}_{2}{ }^{*}\right\rangle$ can now be rewritten as $\nu_{2}\left\langle\mathbf{S}_{1}, \mathbf{S}_{2}\right\rangle+\left\langle\mathbf{S}_{1}, \mathbf{W}_{2}\right\rangle$. Hence,

$$
\begin{aligned}
\mathrm{E} & {\left[\left\langle\mathbf{S}_{1}, \mathbf{U}_{2}{ }^{*}\right\rangle \mid \mathcal{E}_{\mathbf{S}}^{c} \cap \mathcal{E}_{\mathbf{X}_{2}}^{c}\right] } \\
& \stackrel{(a)}{=} \mathrm{E}_{\mathbf{S}_{1}, \mathbf{S}_{2}}\left[\mathrm{E}_{\mathscr{C}_{1}, \mathscr{C}_{2}}\left[\left\langle\mathbf{s}_{1}, \mathbf{U}_{2}{ }^{*}\right\rangle \mid\left(\mathbf{S}_{1}, \mathbf{S}_{2}\right)=\left(\mathbf{s}_{1}, \mathbf{s}_{2}\right), \mathcal{E}_{\mathbf{S}}^{c} \cap \mathcal{E}_{\mathbf{X}_{2}}^{c}\right]\right] \\
& \stackrel{(b)}{=} \mathrm{E}_{\mathbf{S}_{1}, \mathbf{S}_{2}}\left[\mathrm{E}_{\mathscr{C}_{1}, \mathscr{C}_{2}}\left[\nu_{2}\left\langle\mathbf{s}_{1}, \mathbf{s}_{2}\right\rangle \mid\left(\mathbf{S}_{1}, \mathbf{S}_{2}\right)=\left(\mathbf{s}_{1}, \mathbf{s}_{2}\right), \mathcal{E}_{\mathbf{S}}^{c} \cap \mathcal{E}_{\mathbf{X}_{2}}^{c}\right]\right. \\
& +\underbrace{\mathrm{E}_{\mathscr{C}_{1}, \mathscr{C}_{2}}\left[\left\langle\mathbf{s}_{1}, \mathbf{W}_{2}\right\rangle \mid\left(\mathbf{S}_{1}, \mathbf{S}_{2}\right)=\left(\mathbf{s}_{1}, \mathbf{s}_{2}\right), \mathcal{E}_{\mathbf{S}}^{c} \cap \mathcal{E}_{\mathbf{X}_{2}}^{c}\right]}_{=0}] \\
& =\mathrm{E}_{\mathbf{S}_{1}, \mathbf{S}_{2}}\left[\frac{\left\|\mathbf{U}_{2}{ }^{*}\right\|}{\left\|\mathbf{S}_{2}\right\|}\left\langle\mathbf{S}_{1}, \mathbf{S}_{2}\right\rangle \mathrm{E}_{\mathscr{C}_{1}, \mathscr{C}_{2}}\left[\cos \varangle\left(\mathbf{s}_{2}, \mathbf{U}_{2}{ }^{*}\right) \mid\left(\mathbf{S}_{1}, \mathbf{S}_{2}\right)=\left(\mathbf{s}_{1}, \mathbf{s}_{2}\right), \mathcal{E}_{\mathbf{S}}^{c} \cap \mathcal{E}_{\mathbf{X}_{2}}^{c}\right]\right] \\
& \stackrel{(c)}{\geq} \mathrm{E}_{\mathbf{S}_{1}, \mathbf{S}_{2}}\left[\left\|\mathbf{U}_{2}^{*}\right\|\left\|\mathbf{S}_{1}\right\| \cos \varangle\left(\mathbf{S}_{1}, \mathbf{S}_{2}\right) \sqrt{\left.\left(1-2^{-2 R_{2}}\right)(1-\epsilon) \mid \mathcal{E}_{\mathbf{S}}^{c} \cap \mathcal{E}_{\mathbf{X}_{2}}^{c}\right]}\right. \\
& \stackrel{(d)}{\geq} \sqrt{n \sigma^{2}\left(1-2^{-2 R_{2}}\right)} \sqrt{n \sigma^{2}(1-\epsilon)} \rho(1-\epsilon) \sqrt{\left(1-2^{-2 R_{2}}\right)(1-\epsilon)} \\
& \geq n \rho \sigma^{2}\left(1-2^{-2 R_{2}}\right)(1-\epsilon)^{3},
\end{aligned}
$$

where we have denoted by $\mathscr{C}_{i}$ the random codebook of user $i \in\{1,2\}$, and where in (a) we have used law of total expectation, in (b) the second expectation term is zero because for every $\left(\mathbf{s}_{1}, \mathbf{s}_{2}\right) \in \mathcal{E}_{\mathbf{S}}^{c}$

$$
\mathrm{E}_{\mathscr{C}_{2}}\left[\left\langle\mathbf{s}_{1}, \mathbf{W}_{2}\right\rangle \mid\left(\mathbf{S}_{1}, \mathbf{S}_{2}\right)=\left(\mathbf{s}_{1}, \mathbf{s}_{2}\right), \mathcal{E}_{\mathbf{X}_{2}}^{c}\right]=0 .
$$

This holds since in the expectation over the codebooks $\mathscr{C}_{2}$ with conditioning on $\mathcal{E}_{\mathbf{X}_{2}}^{c}$, for every $\mathbf{w}_{2} \in \mathbb{R}^{n}$, the sequences $\mathbf{w}_{2}$ and $-\mathbf{w}_{2}$ are equiprobable, and thus their inner products with $\mathbf{s}_{1}$ cancel off each other. Inequality (c) follows from lower bounding $\cos \varangle\left(\mathbf{s}_{2}, \mathbf{U}_{2}^{*}\right)$ conditioned on $\mathcal{E}_{\mathbf{X}}^{c}$ combined with the fact that conditioned on $\mathcal{E}_{\mathbf{S}}^{c}$ the term $\cos \varangle\left(\mathbf{S}_{1}, \mathbf{S}_{2}\right)$ is positive. Inequality (d) follows from lower bounding $\left\|\mathbf{S}_{1}\right\|$ and $\cos \varangle\left(\mathbf{S}_{1}, \mathbf{S}_{2}\right)$ conditioned on $\mathcal{E}_{\mathbf{S}}^{c}$.

Combining (232) with (233), (235) and (I) gives

$$
\begin{aligned}
\frac{1}{n} \mathrm{E}\left[\left\langle\mathbf{S}_{1}, \mathbf{U}_{2}{ }^{*}\right\rangle\right] \geq & -\sigma^{2}\left(\epsilon+2 \operatorname{Pr}\left[\mathcal{E}_{\mathbf{S}}\right]+(2+\epsilon) \operatorname{Pr}\left[\mathcal{E}_{\mathbf{X}}\right]\right) \\
& +\rho \sigma^{2}\left(1-2^{-2 R_{2}}\right)(1-\epsilon)^{3}\left(1-\left(\operatorname{Pr}\left[\mathcal{E}_{\mathbf{S}}\right]+\operatorname{Pr}\left[\mathcal{E}_{\mathbf{X}}\right]\right)\right) \\
\geq & \rho \sigma^{2}\left(1-2^{-2 R_{2}}\right)(1-\epsilon)^{3}-\sigma^{2}\left(\epsilon+3 \operatorname{Pr}\left[\mathcal{E}_{\mathbf{S}}\right]+(3+\epsilon) \operatorname{Pr}\left[\mathcal{E}_{\mathbf{X}}\right]\right) .
\end{aligned}
$$

Thus, by Lemma 6 and Lemma 8 it follows that for every $\delta>0$ and $0.3>\epsilon>0$ there exists an $n^{\prime}(\delta, \epsilon) \in \mathbb{N}$ such that for all $n>n^{\prime}(\delta, \epsilon)$ 


$$
\frac{1}{n} \mathrm{E}\left[\left\langle\mathbf{S}_{1}, \mathbf{U}_{2}{ }^{*}\right\rangle\right] \geq \rho \sigma^{2}\left(1-2^{-2 R_{2}}\right)(1-\epsilon)^{3}-\sigma^{2}(\epsilon+39 \delta+12 \delta \epsilon) .
$$

Lemma 41 For every $\delta>0$ and $0.3>\epsilon>0$ and every positive integer $n$

$$
\frac{1}{n} \mathrm{E}\left[\left\langle\mathbf{S}_{1}, \mathbf{V}^{*}\right\rangle\right] \geq-\sigma^{2}(12 \delta+3 \epsilon)+\sigma^{2} 2^{-2 R_{1}}\left(1-2^{-2 R_{\mathrm{c}}}\right)(1-2 \epsilon)(1-13 \delta) .
$$

Proof: We begin with the following decomposition:

$$
\frac{1}{n} \mathrm{E}\left[\left\langle\mathbf{S}_{1}, \mathbf{V}^{*}\right\rangle\right]=\frac{1}{n} \mathrm{E}\left[\left\langle\mathbf{U}_{1}^{*}+\mathbf{z}_{\mathrm{Q}_{1}}, \mathbf{V}^{*}\right\rangle\right]=\frac{1}{n}\left(\mathrm{E}\left[\left\langle\mathbf{U}_{1}^{*}, \mathbf{V}^{*}\right\rangle\right]+\mathrm{E}\left[\left\langle\mathbf{z}_{\mathrm{Q}_{1}}, \mathbf{V}^{*}\right\rangle\right]\right) .
$$

The first term on the r.h.s. of (238) is lower bounded as follows:

$$
\begin{aligned}
\frac{1}{n} \mathrm{E}\left[\left\langle\mathbf{U}_{1}^{*}, \mathbf{V}^{*}\right\rangle\right]= & \frac{1}{n} \mathrm{E}\left[\left\langle\mathbf{U}_{1}^{*}, \mathbf{V}^{*}\right\rangle \mid \mathcal{E}_{\mathbf{X}}\right] \operatorname{Pr}\left[\mathcal{E}_{\mathbf{X}}\right]+\frac{1}{n} \mathrm{E}\left[\left\langle\mathbf{U}_{1}^{*}, \mathbf{V}^{*}\right\rangle \mid \mathcal{E}_{\mathbf{X}}^{c}\right] \operatorname{Pr}\left[\mathcal{E}_{\mathbf{X}}^{c}\right] \\
\stackrel{(a)}{\geq} & -\frac{1}{n} \mathrm{E}\left[\left\|\mathbf{U}_{1}^{*}\right\|^{2}+\left\|\mathbf{V}^{*}\right\|^{2} \mid \mathcal{E}_{\mathbf{X}}\right] \operatorname{Pr}\left[\mathcal{E}_{\mathbf{X}}\right] \\
& +\frac{1}{n} \mathrm{E}\left[\left\|\mathbf{U}_{1}^{*}\right\|\left\|\mathbf{V}^{*}\right\|\left|\cos \varangle\left(\mathbf{U}_{1}^{*}, \mathbf{V}^{*}\right)\right| \mathcal{E}_{\mathbf{X}}^{c}\right] \operatorname{Pr}\left[\mathcal{E}_{\mathbf{X}}^{c}\right] \\
\geq & -\frac{1}{n}\left(n \sigma^{2}\left(1-2^{-2 R_{1}}\right)+n \sigma^{2} 2^{-2 R_{1}}\left(1-2^{-2 R_{\mathrm{c}}}\right)\right) \operatorname{Pr}\left[\mathcal{E}_{\mathbf{X}}\right] \\
& +\frac{1}{n} \sqrt{n \sigma^{2}\left(1-2^{-2 R_{1}}\right)} \sqrt{n \sigma^{2} 2^{-2 R_{1}}\left(1-2^{-2 R_{\mathrm{c}}}\right)}(-3 \epsilon) \\
\geq & -\sigma^{2}\left(\operatorname{Pr}\left[\mathcal{E}_{\mathbf{X}}\right]+3 \epsilon\right),
\end{aligned}
$$

where in (a) we have used (234).

We now turn to lower bounding the second term on the r.h.s. of (238).

$$
\begin{aligned}
\frac{1}{n} \mathrm{E}[ & \left.\left\langle\mathbf{z}_{\mathrm{Q}_{1}}, \mathbf{V}^{*}\right\rangle\right] \\
= & \frac{1}{n} \underbrace{\mathbb{E}\left[\left\|\mathbf{z}_{\mathrm{Q}_{1}}\right\|\left\|\mathbf{V}^{*}\right\| \cos \varangle\left(\mathbf{z}_{\mathrm{Q}_{1}}, \mathbf{V}^{*}\right) \mid \mathcal{E}_{\mathbf{S}} \cup \mathcal{E}_{\mathbf{X}}\right]}_{\geq 0} \operatorname{Pr}\left[\mathcal{E}_{\mathbf{S}} \cup \mathcal{E}_{\mathbf{X}}\right] \\
& +\frac{1}{n} \mathrm{E}\left[\left\|\mathbf{z}_{\mathrm{Q}_{1}}\right\|\left\|\mathbf{V}^{*}\right\| \cos \varangle\left(\mathbf{z}_{\mathrm{Q}_{1}}, \mathbf{V}^{*}\right) \mid \mathcal{E}_{\mathbf{S}}^{c} \cap \mathcal{E}_{\mathbf{X}}^{c}\right] \operatorname{Pr}\left[\mathcal{E}_{\mathbf{S}}^{c} \cap \mathcal{E}_{\mathbf{X}}^{c}\right] \\
\geq & \frac{1}{n} \mathrm{E}\left[\left\|\mathbf{z}_{\mathbf{Q}_{1}}\right\|\left\|\mathbf{V}^{*}\right\| \cos \varangle\left(\mathbf{z}_{\mathrm{Q}_{1}}, \mathbf{V}^{*}\right) \mid \mathcal{E}_{\mathbf{S}}^{c} \cap \mathcal{E}_{\mathbf{X}}^{c}\right] \operatorname{Pr}\left[\mathcal{E}_{\mathbf{S}}^{c} \cap \mathcal{E}_{\mathbf{X}}^{c}\right] \\
\geq & \sqrt{\sigma^{2} 2^{-2 R_{1}}(1-\epsilon) \sigma^{2} 2^{-2 R_{1}}\left(1-2^{-2 R_{\mathrm{c}}}\right)\left(1-2^{-2 R_{c}}\right)(1-\epsilon) \operatorname{Pr}\left[\mathcal{E}_{\mathbf{S}}^{c} \cap \mathcal{E}_{\mathbf{X}}^{c}\right]} \\
\geq & \sigma^{2} 2^{-2 R_{1}}\left(1-2^{-2 R_{c}}\right)(1-\epsilon)^{2}\left(1-\operatorname{Pr}\left[\mathcal{E}_{\mathbf{S}} \cup \mathcal{E}_{\mathbf{X}}\right]\right) \\
\geq & \sigma^{2} 2^{-2 R_{1}}\left(1-2^{-2 R_{c}}\right)(1-2 \epsilon)\left(1-\operatorname{Pr}\left[\mathcal{E}_{\mathbf{S}}\right]-\operatorname{Pr}\left[\mathcal{E}_{\mathbf{X}}\right]\right),
\end{aligned}
$$

where in the first equality the first expectation term is non-negative because if $\mathbf{V}^{*}=0$, then it is equal to zero, and if $\mathbf{V}^{*} \neq 0$, then by the conditioning on $\mathcal{E}_{\mathbf{X}}$ it follows that $\cos \varangle\left(\mathbf{z}_{\mathrm{Q}_{1}}, \mathbf{V}^{*}\right)>0$.

Combining (238), with 239) and (1) gives

$$
\frac{1}{n} \mathrm{E}\left[\left\langle\mathbf{S}_{1}, \mathbf{V}^{*}\right\rangle\right] \geq-\sigma^{2}\left(\operatorname{Pr}\left[\mathcal{E}_{\mathbf{X}}\right]+3 \epsilon\right)+\sigma^{2} 2^{-2 R_{1}}\left(1-2^{-2 R_{\mathrm{c}}}\right)(1-2 \epsilon)\left(1-\operatorname{Pr}\left[\mathcal{E}_{\mathbf{S}}\right]-\operatorname{Pr}\left[\mathcal{E}_{\mathbf{X}}\right]\right) .
$$

Thus, by Lemma 6 and Lemma 8 it now follows that for every $\delta>0$ and $0.3>\epsilon>0$ there exists an $n^{\prime}(\delta, \epsilon) \in \mathbb{N}$ such that for all $n>n^{\prime}(\delta, \epsilon)$ 


$$
\frac{1}{n} \mathrm{E}\left[\left\langle\mathbf{S}_{1}, \mathbf{V}^{*}\right\rangle\right] \geq-\sigma^{2}(12 \delta+3 \epsilon)+\sigma^{2} 2^{-2 R_{1}}\left(1-2^{-2 R_{\mathrm{c}}}\right)(1-2 \epsilon)(1-13 \delta) .
$$

Lemma 42 For every $\delta>0$ and $0.3>\epsilon>0$, there exists an $n^{\prime}(\delta, \epsilon) \in \mathbb{N}$ such that for all $n>n^{\prime}(\delta, \epsilon)$

$$
\frac{1}{n} \mathrm{E}\left[\left\langle\mathbf{U}_{1}^{*}, \mathbf{V}^{*}\right\rangle\right] \leq \sigma^{2}(12 \delta+3 \epsilon) .
$$

Proof:

$$
\begin{aligned}
\frac{1}{n} \mathrm{E}\left[\left\langle\mathbf{U}_{1}^{*}, \mathbf{V}^{*}\right\rangle\right]= & \frac{1}{n} \mathrm{E}\left[\left\langle\mathbf{U}_{1}^{*}, \mathbf{V}^{*}\right\rangle \mid \mathcal{E}_{\mathbf{X}}\right] \operatorname{Pr}\left[\mathcal{E}_{\mathbf{X}}\right]+\frac{1}{n} \mathrm{E}\left[\left\langle\mathbf{U}_{1}^{*}, \mathbf{V}^{*}\right\rangle \mid \mathcal{E}_{\mathbf{X}}^{c}\right] \operatorname{Pr}\left[\mathcal{E}_{\mathbf{X}}^{c}\right] \\
\leq & \frac{1}{n} \mathrm{E}\left[\left\|\mathbf{U}_{1}^{*}\right\|\left\|\mathbf{V}^{*}\right\| \mid \mathcal{E}_{\mathbf{X}}\right] \operatorname{Pr}\left[\mathcal{E}_{\mathbf{X}}\right]+\frac{1}{n} \mathrm{E}\left[\left\|\mathbf{U}_{1}^{*}\right\|\left\|\mathbf{V}^{*}\right\|\left|\cos \varangle\left(\mathbf{U}_{1}^{*}, \mathbf{V}^{*}\right)\right| \mathcal{E}_{\mathbf{X}}^{c}\right] \operatorname{Pr}\left[\mathcal{E}_{\mathbf{X}}^{c}\right] \\
\leq & \frac{1}{n} \sqrt{n \sigma^{2}\left(1-2^{-2 R_{1}}\right)} \sqrt{n \sigma^{2} 2^{-2 R_{1}}\left(1-2^{-2 R_{\mathrm{c}}}\right)} \operatorname{Pr}\left[\mathcal{E}_{\mathbf{X}}\right] \\
& +\frac{1}{n} \sqrt{n \sigma^{2}\left(1-2^{-2 R_{1}}\right)} \sqrt{n \sigma^{2} 2^{-2 R_{1}}\left(1-2^{-2 R_{\mathrm{c}}}\right)}(3 \epsilon) \\
\leq & \sigma^{2}\left(\operatorname{Pr}\left[\mathcal{E}_{\mathbf{X}}\right]+3 \epsilon\right) .
\end{aligned}
$$

Thus, it follows by Lemma 8 that for every $\delta>0$ and $0.3>\epsilon>0$ there exists an $n^{\prime}(\delta, \epsilon) \in \mathbb{N}$ such that for all $n>n^{\prime}(\delta, \epsilon)$

$$
\frac{1}{n} \mathrm{E}\left[\left\langle\mathbf{U}_{1}^{*}, \mathbf{V}^{*}\right\rangle\right] \leq \sigma^{2}(12 \delta+3 \epsilon) .
$$

Lemma 43 For every $\delta>0$ and $0.3>\epsilon>0$, there exists an $n^{\prime}(\delta, \epsilon) \in \mathbb{N}$ such that for all $n>n^{\prime}(\delta, \epsilon)$

$$
\frac{1}{n} \mathrm{E}\left[\left\langle\mathbf{U}_{2}^{*}, \mathbf{V}^{*}\right\rangle\right] \leq \sigma^{2} 11 \delta+\rho \sigma^{2} 2^{-2 R_{1}}\left(1-2^{-2 R_{2}}\right)\left(1-2^{-2 R_{\mathrm{c}}}\right)(1+7 \epsilon) .
$$

Proof:

$$
\begin{aligned}
\frac{1}{n} \mathrm{E}\left[\left\langle\mathbf{U}_{2}^{*}, \mathbf{V}^{*}\right\rangle\right]= & \frac{1}{n} \mathrm{E}\left[\left\langle\mathbf{U}_{2}^{*}, \mathbf{V}^{*}\right\rangle \mid \mathcal{E}_{\mathbf{X}}\right] \operatorname{Pr}\left[\mathcal{E}_{\mathbf{X}}\right]+\frac{1}{n} \mathrm{E}\left[\left\langle\mathbf{U}_{2}^{*}, \mathbf{V}^{*}\right\rangle \mid \mathcal{E}_{\mathbf{X}}^{c}\right] \operatorname{Pr}\left[\mathcal{E}_{\mathbf{X}}^{c}\right] \\
\leq & \frac{1}{n} \mathrm{E}\left[\left\|\mathbf{U}_{2}^{*}\right\|\left\|\mathbf{V}^{*}\right\| \mid \mathcal{E}_{\mathbf{X}}\right] \operatorname{Pr}\left[\mathcal{E}_{\mathbf{X}}\right]+\frac{1}{n} \mathrm{E}\left[\left\|\mathbf{U}_{2}^{*}\right\|\left\|\mathbf{V}^{*}\right\|\left|\cos \varangle\left(\mathbf{U}_{2}^{*}, \mathbf{V}^{*}\right)\right| \mathcal{E}_{\mathbf{X}}^{c}\right] \operatorname{Pr}\left[\mathcal{E}_{\mathbf{X}}^{c}\right] \\
\leq & \sqrt{\sigma^{2}\left(1-2^{-2 R_{2}}\right) \sigma^{2} 2^{-2 R_{1}}\left(1-2^{-2 R_{\mathrm{c}}}\right)} \operatorname{Pr}\left[\mathcal{E}_{\mathbf{X}}\right] \\
& +\sqrt{\sigma^{2}\left(1-2^{-2 R_{2}}\right) \sigma^{2} 2^{-2 R_{1}}\left(1-2^{-2 R_{\mathrm{c}}}\right)} \bar{\rho}(1+7 \epsilon) \\
\leq & \sigma^{2} \operatorname{Pr}\left[\mathcal{E}_{\mathbf{X}}\right]+\rho \sigma^{2} 2^{-2 R_{1}}\left(1-2^{-2 R_{2}}\right)\left(1-2^{-2 R_{\mathrm{c}}}\right)(1+7 \epsilon) .
\end{aligned}
$$

Thus, it follows by Lemma 8 that for every $\delta>0$ and $0.3>\epsilon>0$ there exists an $n^{\prime}(\delta, \epsilon) \in \mathbb{N}$ such that for all $n>n^{\prime}(\delta, \epsilon)$

$$
\frac{1}{n} \mathrm{E}\left[\left\langle\mathbf{U}_{2}^{*}, \mathbf{V}^{*}\right\rangle\right] \leq \sigma^{2} 11 \delta+\rho \sigma^{2} 2^{-2 R_{1}}\left(1-2^{-2 R_{2}}\right)\left(1-2^{-2 R_{\mathfrak{c}}}\right)(1+7 \epsilon) .
$$


The distortion $D_{1}$ of the genie-aided scheme is now upper bounded as follows:

$$
\begin{aligned}
D_{1}= & \frac{1}{n} \mathrm{E}\left[\left\|\mathbf{S}_{1}-\hat{\mathbf{S}}_{1}^{\mathrm{G}}\right\|^{2}\right] \\
= & \sigma^{2}-2 \gamma_{1,1} \frac{1}{n} \mathrm{E}\left[\left\langle\mathbf{S}_{1}, \mathbf{U}_{1}{ }^{*}\right\rangle\right]-2 \gamma_{1,2} \frac{1}{n} \mathrm{E}\left[\left\langle\mathbf{S}_{1}, \mathbf{U}_{2}{ }^{*}\right\rangle\right]-2 \gamma_{1,3} \frac{1}{n} \mathrm{E}\left[\left\langle\mathbf{S}_{1}, \mathbf{V}^{*}\right\rangle\right] \\
& +\gamma_{1,1}{ }^{2} \sigma^{2}\left(1-2^{-2 R_{1}}\right)+2 \gamma_{1,1} \gamma_{1,2} \frac{1}{n} \mathrm{E}\left[\left\langle\mathbf{U}_{1}{ }^{*}, \mathbf{U}_{2}{ }^{*}\right\rangle\right]+\gamma_{1,2}{ }^{2} \sigma^{2}\left(1-2^{-2 R_{2}}\right) \\
& +2 \gamma_{1,1} \gamma_{1,3} \frac{1}{n} \mathrm{E}\left[\left\langle\mathbf{U}_{1}{ }^{*}, \mathbf{V}^{*}\right\rangle\right]+2 \gamma_{1,2} \gamma_{1,3} \frac{1}{n} \mathrm{E}\left[\left\langle\mathbf{U}_{2}{ }^{*}, \mathbf{V}^{*}\right\rangle\right]+\gamma_{1,3}{ }^{2} \sigma^{2} 2^{-2 R_{1}}\left(1-2^{-2 R_{\mathrm{c}}}\right) \\
& \stackrel{(a)}{\leq} \sigma^{2} 2^{-2\left(R_{1}+R_{\mathrm{c}}\right)} \frac{1-\rho^{2}\left(1-2^{-2 R_{2}}\right)}{1-\rho^{2}\left(1-2^{-2 R_{2}}\right)\left(1-2^{-2\left(R_{1}+R_{\mathrm{c}}\right)}\right)}+\xi^{\prime}(\delta, \epsilon),
\end{aligned}
$$

where in (a) we have used Lemma 38, Lemma 39, Lemma 40, Lemma 41, Lemma 42, Lemma 43 and Lemma 11 and where $\lim _{\delta, \epsilon \rightarrow 0} \xi^{\prime}(\delta, \epsilon)=0$.

Now we upper-bound $D_{2}$. By Corollary 4 it suffices to analyze the genie-aided scheme. Since $\hat{\mathbf{S}}_{2}^{\mathrm{G}}=\gamma_{2,1} \mathbf{U}_{1}{ }^{*}+\gamma_{2,2} \mathbf{U}_{2}{ }^{*}+\gamma_{2,3} \mathbf{V}^{*}$, we have

$$
\begin{aligned}
D_{2}= & \frac{1}{n} \mathrm{E}\left[\left\|\mathbf{S}_{2}-\hat{\mathbf{S}}_{2}^{\mathrm{G}}\right\|^{2}\right]=\frac{1}{n} \mathrm{E}\left[\left\|\mathbf{S}_{2}-\left(\gamma_{2,1} \mathbf{U}_{1}{ }^{*}+\gamma_{2,2} \mathbf{U}_{2}{ }^{*}+\gamma_{2,3} \mathbf{V}^{*}\right)\right\|^{2}\right] \\
= & \frac{1}{n}\left(\mathrm{E}\left[\left\|\mathbf{S}_{2}\right\|^{2}\right]-2 \gamma_{2,1} \mathrm{E}\left[\left\langle\mathbf{S}_{2}, \mathbf{U}_{1}{ }^{*}\right\rangle\right]-2 \gamma_{2,2} \mathrm{E}\left[\left\langle\mathbf{S}_{2}, \mathbf{U}_{2}{ }^{*}\right\rangle\right]-2 \gamma_{2,3} \mathrm{E}\left[\left\langle\mathbf{S}_{2}, \mathbf{V}^{*}\right\rangle\right]\right. \\
& +\gamma_{2,1}{ }^{2} \mathrm{E}\left[\left\|\mathbf{U}_{1}{ }^{*}\right\|^{2}\right]+2 \gamma_{2,1} \gamma_{2,2} \mathrm{E}\left[\left\langle\mathbf{U}_{1}{ }^{*}, \mathbf{U}_{2}{ }^{*}\right\rangle\right]+\gamma_{2,2}{ }^{2} \mathrm{E}\left[\left\|\mathbf{U}_{2}{ }^{*}\right\|^{2}\right] \\
& \left.+2 \gamma_{2,1} \gamma_{2,3} \mathrm{E}\left[\left\langle\mathbf{U}_{1}{ }^{*}, \mathbf{V}^{*}\right\rangle\right]+2 \gamma_{2,2} \gamma_{2,3} \mathrm{E}\left[\left\langle\mathbf{U}_{2}{ }^{*}, \mathbf{V}^{*}\right\rangle\right]+\gamma_{2,3}{ }^{2} \mathrm{E}\left[\left\|\mathbf{V}^{*}\right\|^{2}\right]\right) \\
= & \sigma^{2}-2 \gamma_{2,1} \frac{1}{n} \mathrm{E}\left[\left\langle\mathbf{S}_{2}, \mathbf{U}_{1}{ }^{*}\right\rangle\right]-2 \gamma_{2,2} \frac{1}{n} \mathrm{E}\left[\left\langle\mathbf{S}_{2}, \mathbf{U}_{2}{ }^{*}\right\rangle\right]-2 \gamma_{2,3} \frac{1}{n} \mathrm{E}\left[\left\langle\mathbf{S}_{2}, \mathbf{V}^{*}\right\rangle\right] \\
& +\gamma_{2,1}{ }^{2}\left(\sigma^{2}\left(1-2^{-2 R_{1}}\right)\right)+2 \gamma_{2,1} \gamma_{2,2} \frac{1}{n} \mathrm{E}\left[\left\langle\mathbf{U}_{1}{ }^{*}, \mathbf{U}_{2}{ }^{*}\right\rangle\right]+\gamma_{2,2}{ }^{2}\left(\sigma^{2}\left(1-2^{-2 R_{2}}\right)\right) \\
& +2 \gamma_{2,1} \gamma_{2,3} \frac{1}{n} \mathrm{E}\left[\left\langle\mathbf{U}_{1}{ }^{*}, \mathbf{V}^{*}\right\rangle\right]+2 \gamma_{2,2} \gamma_{2,3} \frac{1}{n} \mathrm{E}\left[\left\langle\mathbf{U}_{2}{ }^{*}, \mathbf{V}^{*}\right\rangle\right]+\gamma_{2,3}{ }^{2}\left(\sigma^{2} 2^{-2 R_{1}}\left(1-2^{-2 R_{\mathrm{c}}}\right)\right),
\end{aligned}
$$

where in the last equality all expected squared norms have been replaced by their explicit values, i.e. $\mathrm{E}\left[\left\|\mathbf{S}_{2}\right\|^{2}\right]=n \sigma^{2}$, and $\mathrm{E}\left[\left\|\mathbf{U}_{i}\right\|^{2}\right]=n \sigma^{2}\left(1-2^{-2 R_{i}}\right)$ for $i \in\{1,2\}$ and $\mathrm{E}\left[\|\mathbf{V}\|^{2}\right]=$ $n \sigma^{2} 2^{-2 R_{1}}\left(1-2^{-2 R_{\mathrm{c}}}\right)$. The remaining expectations of the inner products are bounded in the following three lemmas.

Lemma 44 For every $\delta>0$ and $0.3>\epsilon>0$ and every positive integer $n$

$$
\frac{1}{n} \mathrm{E}\left[\left\langle\mathbf{S}_{2}, \mathbf{U}_{2}^{*}\right\rangle\right] \geq \sigma^{2}\left(1-2^{-2 R_{2}}\right)(1-2 \epsilon)(1-13 \delta) .
$$

Proof:

$$
\begin{aligned}
\frac{1}{n} \mathrm{E}\left[\left\langle\mathbf{S}_{2}, \mathbf{U}_{2}{ }^{*}\right\rangle\right]= & \frac{1}{n} \underbrace{\mathrm{E}\left[\left\|\mathbf{S}_{2}\right\|\left\|\mathbf{U}_{2}{ }^{*}\right\| \cos \varangle\left(\mathbf{S}_{2}, \mathbf{U}_{2}{ }^{*}\right) \mid \mathcal{E}_{\mathbf{S}} \cup \mathcal{E}_{\mathbf{X}}\right]}_{\geq 0} \operatorname{Pr}\left[\mathcal{E}_{\mathbf{S}} \cup \mathcal{E}_{\mathbf{X}}\right] \\
& +\frac{1}{n} \mathrm{E}\left[\left\|\mathbf{S}_{2}\right\|\left\|\mathbf{U}_{2}{ }^{*}\right\| \cos \varangle\left(\mathbf{S}_{2}, \mathbf{U}_{2}{ }^{*}\right) \mid \mathcal{E}_{\mathbf{S}}^{c} \cap \mathcal{E}_{\mathbf{X}}^{c}\right] \operatorname{Pr}\left[\mathcal{E}_{\mathbf{S}}^{c} \cap \mathcal{E}_{\mathbf{X}}^{c}\right] \\
\geq & \frac{1}{n} \mathrm{E}\left[\left\|\mathbf{S}_{2}\right\|\left\|\mathbf{U}_{2}{ }^{*}\right\| \cos \varangle\left(\mathbf{S}_{2}, \mathbf{U}_{2}{ }^{*}\right) \mid \mathcal{E}_{\mathbf{S}}^{c} \cap \mathcal{E}_{\mathbf{X}}^{c}\right] \operatorname{Pr}\left[\mathcal{E}_{\mathbf{S}}^{c} \cap \mathcal{E}_{\mathbf{X}}^{c}\right] \\
\geq & \sqrt{\sigma^{2}(1-\epsilon) \sigma^{2}\left(1-2^{-2 R_{2}}\right)\left(1-2^{-2 R_{2}}\right)}(1-\epsilon) \operatorname{Pr}\left[\mathcal{E}_{\mathbf{S}}^{c} \cap \mathcal{E}_{\mathbf{X}}^{c}\right]
\end{aligned}
$$




$$
\begin{aligned}
& \geq \sigma^{2}\left(1-2^{-2 R_{2}}\right)(1-\epsilon)^{2}\left(1-\operatorname{Pr}\left[\mathcal{E}_{\mathbf{S}} \cup \mathcal{E}_{\mathbf{X}}\right]\right) \\
& \geq \sigma^{2}\left(1-2^{-2 R_{2}}\right)(1-2 \epsilon)\left(1-\operatorname{Pr}\left[\mathcal{E}_{\mathbf{S}}\right]-\operatorname{Pr}\left[\mathcal{E}_{\mathbf{X}}\right]\right),
\end{aligned}
$$

where in the first equality the first expectation term is non-negative because if $\mathbf{U}_{2}^{*}=0$, then it is equal to zero, and if $\mathbf{U}_{2}^{*} \neq 0$, then by the conditioning on $\mathcal{E}_{\mathbf{X}}$ it follows that $\cos \varangle\left(\mathbf{S}_{2}, \mathbf{U}_{2}{ }^{*}\right)>0$.

By Lemma 6 and Lemma 8 it now follows that for every $\delta>0$ and $0.3>\epsilon>0$ there exists an $n^{\prime}(\delta, \epsilon) \in \mathbb{N}$ such that for all $n>n^{\prime}(\delta, \epsilon)$

$$
\frac{1}{n} \mathrm{E}\left[\left\langle\mathbf{S}_{2}, \mathbf{U}_{2}{ }^{*}\right\rangle\right] \geq \sigma^{2}\left(1-2^{-2 R_{2}}\right)(1-2 \epsilon)(1-13 \delta) .
$$

Lemma 45 For every $\delta>0$ and $0.3>\epsilon>0$ there exists an $n^{\prime}(\delta, \epsilon) \in \mathbb{N}$ such that for all $n>n^{\prime}(\delta, \epsilon)$

$$
\frac{1}{n} \mathrm{E}\left[\left\langle\mathbf{S}_{2}^{*}, \mathbf{U}_{1}^{*}\right\rangle\right] \geq \rho \sigma^{2}\left(1-2^{-2 R_{1}}\right)(1-\epsilon)^{3}-\sigma^{2}(\epsilon+39 \delta+12 \delta \epsilon) .
$$

Proof: The proof is following in a similar manner as the proof of Lemma 40 .

Lemma 46 For every $\delta>0$ and $0.3>\epsilon>0$ and every positive integer $n$

$$
\frac{1}{n} \mathrm{E}\left[\left\langle\mathbf{S}_{2}, \mathbf{V}^{*}\right\rangle\right] \geq-\rho \sigma^{2}(12 \delta+3 \epsilon)+\rho \sigma^{2} 2^{-2 R_{1}}\left(1-2^{-2 R_{\mathrm{c}}}\right)(1-2 \epsilon)(1-13 \delta) .
$$

Proof: We begin with the following decomposition.

$$
\begin{aligned}
\frac{1}{n} \mathrm{E}\left[\left\langle\mathbf{S}_{2}, \mathbf{V}^{*}\right\rangle\right] & =\frac{1}{n} \mathrm{E}\left[\left\langle\rho \mathbf{S}_{1}+\mathbf{Z}_{G_{2}}, \mathbf{V}^{*}\right\rangle\right] \\
& =\frac{1}{n}\left(\rho \mathrm{E}\left[\left\langle\mathbf{S}_{1}, \mathbf{V}^{*}\right\rangle\right]+\mathrm{E}\left[\left\langle\mathbf{Z}_{G_{2}}, \mathbf{V}^{*}\right\rangle\right]\right) .
\end{aligned}
$$

The second term on the r.h.s. of (243) vanishes as follows:

$$
\mathrm{E}\left[\left\langle\mathbf{Z}_{G_{2}}, \mathbf{V}^{*}\right\rangle\right]=\mathrm{E}_{\mathbf{S}_{1}, \mathscr{C}_{1}, \mathscr{C}_{c}}\left[\mathrm{E}_{\mathbf{S}_{2}}\left[\left\langle\mathbf{Z}_{G_{2}}, \mathbf{v}^{*}\right\rangle \mid \mathbf{S}_{1}=\mathbf{s}_{1}, \mathscr{C}_{1}=\mathcal{C}_{1}, \mathscr{C}_{c}=\mathcal{C}_{c}\right]\right]=0 .
$$

This holds, since conditionally on $\mathbf{S}_{1}$ the random variable $\mathbf{Z}_{G_{2}}$ is independent of $\left(\mathbf{S}_{1}, \mathbf{V}^{*}\right)$, and therefore in the expectation over $\mathbf{S}_{2}$, for every $\mathbf{z}_{\mathrm{G}_{2}} \in \mathbb{R}$, the sequences $\mathbf{z}_{\mathrm{G}_{2}}$ and $-\mathbf{z}_{\mathrm{G}_{2}}$ are equiprobable and thus their inner products with $\mathbf{v}^{*}$ cancel off each other.

The distortion $D_{2}$ of the genie-aided scheme is now upper bounded as follows:

$$
\begin{aligned}
D_{2}= & \frac{1}{n} \mathrm{E}\left[\left\|\mathbf{S}_{2}-\hat{\mathbf{S}}_{2}^{\mathrm{G}}\right\|^{2}\right] \\
= & \sigma^{2}-2 \gamma_{2,1} \frac{1}{n} \mathrm{E}\left[\left\langle\mathbf{S}_{2}, \mathbf{U}_{1}{ }^{*}\right\rangle\right]-2 \gamma_{2,2} \frac{1}{n} \mathrm{E}\left[\left\langle\mathbf{S}_{2}, \mathbf{U}_{2}{ }^{*}\right\rangle\right]-2 \gamma_{2,3} \frac{1}{n} \mathrm{E}\left[\left\langle\mathbf{S}_{2}, \mathbf{V}^{*}\right\rangle\right] \\
& +\gamma_{2,1}{ }^{2}\left(\sigma^{2}\left(1-2^{-2 R_{1}}\right)\right)+2 \gamma_{2,1} \gamma_{2,2} \frac{1}{n} \mathrm{E}\left[\left\langle\mathbf{U}_{1}{ }^{*}, \mathbf{U}_{2}{ }^{*}\right\rangle\right]+\gamma_{2,2}{ }^{2}\left(\sigma^{2}\left(1-2^{-2 R_{2}}\right)\right) \\
& +2 \gamma_{2,1} \gamma_{2,3} \frac{1}{n} \mathrm{E}\left[\left\langle\mathbf{U}_{1}{ }^{*}, \mathbf{V}^{*}\right\rangle\right]+2 \gamma_{2,2} \gamma_{2,3} \frac{1}{n} \mathrm{E}\left[\left\langle\mathbf{U}_{2}{ }^{*}, \mathbf{V}^{*}\right\rangle\right]+\gamma_{2,3}{ }^{2}\left(\sigma^{2} 2^{-2 R_{1}}\left(1-2^{-2 R_{\mathrm{c}}}\right)\right) \\
& \stackrel{(a)}{\leq} \sigma^{2} 2^{-2 R_{2}} \frac{1-\rho^{2}\left(1-2^{-2\left(R_{1}+R_{\mathrm{c}}\right)}\right)}{1-\rho^{2}\left(1-2^{-2 R_{2}}\right)\left(1-2^{-2\left(R_{1}+R_{\mathrm{c}}\right)}\right)}+\xi^{\prime}(\delta, \epsilon),
\end{aligned}
$$

where in (a) we have used Lemma39, Lemma42, Lemma43, Lemma 44, Lemma45, Lemma46 and Lemma 11 and where $\lim _{\delta, \epsilon \rightarrow 0} \xi^{\prime}(\delta, \epsilon)=0$. 


\section{REFERENCES}

[1] A. Lapidoth and S. Tinguely, "Sending a bivariate Gaussian over a Gaussian MAC," IEEE Trans. Inform. Theory, vol. IT-56, no. 6, pp. 2714-2752, June 2010.

[2] A. Lapidoth and S. Tinguely, "Sending a bivariate Gaussian source over a Gaussian MAC with feedback," IEEE Trans. Inform. Theory, vol. IT-56, no. 4, pp. 1852-1864, April 2010.

[3] T. M. Cover, A. El Gamal and M. Salehi, "Multiple access channels with arbitrarily correlated sources," IEEE Trans. Inform. Theory, vol. IT-26, no. 6, pp. 648-657, Nov. 1980.

[4] D. Slepian and J. K. Wolf, "Noiseless coding of correlated information sources", IEEE Trans. Inform. Theory, vol. IT-19, no. 4, pp. 471-480, July 1973.

[5] K. De Bruyn, V. V. Perlov and E. C. Van der Meulen, "Reliable transmission of correlated sources over an asymmetric multiple-access channel," IEEE Trans. Inform. Theory, vol. IT-33, no. 5, pp. 716-718, Sep. 1987.

[6] D. Gunduz and E. Erkip, "Correlated sources over an asymmetric MAC with one distortion criterion," CISS, Baltimore, MD, March 2007.

[7] J. Xiao and Z. Luo, "Compression of Gaussian correlated sources under individual distortion criteria," Proceedings 43rd Allerton Conference, Illinois, Sep. 2005.

[8] A. D. Wyner and J. Ziv, "The rate-distortion function for source coding with side information at the decoder," IEEE Trans. Inform. Theory, vol. IT-22, no. 1, pp. 1-10, Jan. 1976.

[9] A. H. Kaspi and T. Berger, "Rate-distortion for correlated sources with partially separated encoders", IEEE Trans. Inform. Theory, vol. 28, no. 6, pp. 828-840, Sep. 1982.

[10] S. I. Bross, A. Lapidoth and M. A. Wigger, "The Gaussian MAC with conferencing encoders," in Proceedings IEEE International Symposium on Information Theory, pp. 2702-2706, July 6-11 2008.

[11] S. I. Bross, A. Lapidoth, and M. Wigger, "Dirty-paper coding for the Gaussian multiaccess channel with conferencing", IEEE Trans. Inform. Theory, vol. 58, no. 9, Sep. 2012.

[12] F. M. J. Willems, "The discrete memoryless multiple access channel with partially cooperating encoders," IEEE Trans. Inform. Theory, vol. IT-29, no. 3, pp. 441-445, May 1983.

[13] Y. Oohama, "Gaussian multiterminal source coding," IEEE Trans. Inform. Theory, vol. IT-43, No. 6, pp. 19121923, Nov. 1997.

[14] A. B. Wagner, S. Tavildar and P. Viswanath, "The rate region of the quadratic Gaussian two-terminal sourcecoding problem," IEEE Trans. Inform. Theory, vol. IT-54, no. 5, pp. 1938-1961, May 2008.

[15] T. M. Cover and J. A. Thomas, "Elements of Information Theory," Wiley, 1991.

[16] L. H. Ozarow, "The capacity of the white Gaussian MAC with feedback," IEEE Trans. Inform. Theory, vol. IT-30, no. 4, pp. 623-629, July 1985.

[17] M. A. Wigger, "Cooperation on the multiple-access channel," Ph.D. Thesis, ETH, Zürich, Switzerland, 2008.

[18] H. S. Witsenhausen, "On sequences of pairs of dependent random variables," SIAM Journal on Applied Mathematics, vol. 28, no. 1, pp. 100-113, January 1975.

[19] Y. A. Rozanov (translated by A. Feinstein), "Stationary Random Processes," Holden-Day, 1967.

[20] W. Bryc, A. Dembo and A. Kagan, "On the maximum correlation coefficient," SIAM Journal on Theory of Probability and its Applications, vol. 49, no. 1, pp. 132-138, 2005.

[21] C. E. Shannon "Probability of error for optimal codes in a Gaussian channel", Bell System Technical Journal, vol. 38, pp. 611-656, May 1959.

[22] A. D. Wyner, "Random packings and coverings of the unit $n$-sphere", Bell System Technical Journal, vol. 46, pp. 2111-2118, November 1967.

[23] R. L. Graham, D. E. Knuth, and O. Patashnik, Concrete Mathematics: "A Foundation for Computer Science", 2nd ed. Addison-Wesley, 1994.

[24] A. El Gamal and Y. H. Kim, Network Information Theory, Cambridge University Press, 2012. 